\title{
Modeling and Optimizing Content-based Publish/Subscribe Systems
}

\author{
vorgelegt von \\ Diplom-Ingenieur \\ Arnd Schröter \\ von der Fakultät IV - Elektrotechnik und Informatik \\ der Technischen Universität Berlin \\ zur Erlangung des akademischen Grades \\ Doktor der Ingenieurwissenschaften \\ - Dr.-Ing. - \\ genehmigte Dissertation \\ Promotionsausschuss: \\ Vorsitzender: Professor Dr. Odej Kao \\ Gutachter: Professor Dr. Hans-Ulrich Heiß \\ Gutachter: Professor Dr. Gero Mühl
}

Tag der wissenschaftlichen Aussprache:

9. August 2012

Berlin 2012

D 83 



\begin{abstract}
Publish/subscribe systems are increasingly often used as a communication platform for loosely-coupled systems. Its applications are widespread ranging from real-time business applications and eHome scenarios to Internet applications, such as communication and collaboration platforms or social networks. The performance of publish/subscribe systems plays an essential role for their acceptance. Especially, in mission-critical and financial areas, the system should guarantee certain predefined performance metrics such as throughput and latency. Furthermore, due to the economic pressure operators are forced to run publish/subscribe systems as efficiently as possible to reduce their operating costs. These requirements lead to the necessity to develop strategies for planning and managing publish/subscribe systems. Therefore, in this thesis we present a holistic approach targeting the modeling and optimization of the entire life cycle of publish/subscribe systems including the design, the runtime and the redesign phase. We show how an optimized infrastructure meeting predefined requirements can be determined before the system is deployed and how this approach can be used for a redesign. This allows designers to decide about the required size and capabilities of the infrastructure they have to set up. The foundation of this capacity planning is a stochastic system model that covers all important inputs and outputs. It allows a detailed analysis of distributed content-based publish/subscribe systems. We show the applicability of the approach with the help of an extensive case study. A second main topic of this thesis is the online optimization of publish/subscribe systems that avoids a performance degradation at runtime by adapting to dynamic changes of the environment. This approach is complementary to capacity planning. The algorithm works on the message routing level in order to reduce the message overhead. It is based on a new class of routing algorithms called hybrid routing which is a generalization of standard algorithms. Apart from the evaluation, we also meet challenges in fault-tolerance and in the composition with other adaptive algorithms.
\end{abstract}




\section{Zusammenfassung}

Publish/Subscribe-Systeme werden zunehmend als Kommunikationsplattform für lose-gekoppelte, verteilte Systeme verwendet. Dabei erstrecken sich die Anwendungsbereiche von Echtzeit-Anwendungen in Unternehmen, über eHomeSzenarien bis hin zu Kommunikations- und Kollaborationsplattformen im Internet wie z.B. soziale Netzwerke. Für die Akzeptanz von Publish/SubscribeSystemen spielt die Leistungsfähigkeit eine wichtige Rolle. Dies gilt vor allem für missionskritische Anwendungen und im Finanzbereich von Unternehmen. Bei diesen Anwendungen ist es wichtig, dass das System definierte Leistungswerte wie Durchsatz und Latenz einhält. Gleichzeitig hat der Betreiber das Ziel, das Publish/Subscribe-System mit möglichst geringen Kosten zu betreiben. Diese gegensätzlichen Anforderungen an das System führen zur Notwendigkeit einer Kapazitätsplanung. Dazu wird in dieser Arbeit ein ganzheitlicher Ansatz vorgestellt, der den kompletten Lebenszyklus eines Publish/Subscribe-Systems mit Designphase, Betrieb und Redesignphase umfasst. Es wird gezeigt, wie vor Inbetriebnahme aus vorgegebenen Anforderungen an das System eine passende Konfiguration abgeleitet werden kann. Das zugrunde liegende stochastische Systemmodell berücksichtigt alle wichtigen Einflussgrößen und erlaubt eine detaillierte Analyse verteilter inhaltsbasierter Publish/Subscribe-Systeme. Anhand einer Fallstudie wird die Anwendbarkeit der vorgestellten Kapazitätsplanung gezeigt. Der zweite Schwerpunkt der Arbeit ist die Laufzeitoptimierung von Publish/ Subscribe-Systemen, um auf Änderungen der Umgebung geeignet reagieren zu können. Dieser Ansatz ist komplementär zur Kapazitätsplanung und ergänzt diese. Der vorgestellte Online-Algorithmus wirkt dabei auf der Ebene des Routings von Nachrichten und hat das Ziel, den Nachrichtenoverhead zu reduzieren. Er basiert auf einer verallgemeinerten Klasse bekannter Routingalgorithmen. Neben einer ausführlichen Evaluation wird der vorgestellte Algorithmus auch hinsichtlich Fehlertoleranz und Komponierbarkeit mit anderen Algorithmen untersucht. 


\section{Preface}

\section{Acknowledgements}

This PhD thesis is the biggest challenge I have ever accepted. Although its completion is an individual task, such a project cannot be done without the direct and indirect help of many people around me. First of all, I thank my advisors Prof. Hans-Ulrich Heiß and Prof. Gero Mühl that gave me the motivating environment to start, to get on and to complete this thesis. Furthermore, I am grateful for the fruitful discussions and inspirations given by my colleagues Dr. Jan Richling, Helge Parzyjegla and Daniel Graff. Finally, I thank the Bundesamt für Sicherheit in der Informationstechnik (BSI) and the Deutsche Forschungsgemeinschaft (DFG) for funding projects that were closely related to my research topic. Last but not least, many thanks to my girlfriend, family and closest friends that always kept my grounded during this time.

\section{Publications}

Parts of this thesis are based on papers published during the work on it. Chapter 4 is partly based on a joint work with Gero Mühl, Samuel Kounev, Helge Parzyjegla and Jan Richling [93]. Chapter 6 is based on a joint work with Daniel Graff, Gero Mühl, Jan Richling and Helge Parzyjegla [92]. A number of other co-authored publications are only partly included in this thesis $[68,73,74,75,76,91,94]$. 


\section{Contents}

1 Introduction $\quad 1$

1.1 Motivation .................... 1

1.2 Shortcomings of Current Approaches . . . . . . . . . . . 5

1.3 Contributions of this Thesis . . . . . . . . . . . . 7

1.4 Organization of this Thesis ............. 8

2 Distributed Publish/Subscribe Systems 11

2.1 Introduction . . . . . . . . . . . . . . . . . . 11

2.2 System Model . . . . . . . . . . . . . . . . . . . 12

2.3 System Architecture . . . . . . . . . . . . . . . . . . 12

2.4 Matching and Routing ................. . . 14

2.4.1 Data and Filter Model . . . . . . . . . . . . . . 14

2.4 .2 Correctness . . . . . . . . . . . . . . 15

2.4.3 Matching and Forwarding . . . . . . . . . . . . . 16

2.4 .4 Notification Routing . . . . . . . . . . . . . . 16

2.5 Basic Assumptions . . . . . . . . . . . . . . . . . 19

3 Optimizing Publish/Subscribe Systems 21

3.1 Introduction . . . . . . . . . . . . . . . . . . . 21

3.2 Requirements on Publish/Subscribe Systems . . . . . . . . . . . 22

3.2.1 Requirements of the Clients . . . . . . . . . . . . . 22

3.2.2 Requirements of the Operators . . . . . . . . . . 23

3.3 Optimization Objectives ................. 24

3.4 Optimizations within the Life Cycle . . . . . . . . . . . . 26

3.5 Capacity Planning vs. Adaptive Algorithms . . . . . . . . . . . . 28

4 Stochastic System Model 31

4.1 Introduction . . . . . . . . . . . . . . . . . . . 32

4.2 Related Work . . . . . . . . . . . . . . . . . 33

4.3 Inputs and Outputs of the System Model . . . . . . . . . . . . 35

4.3.1 System Outputs . . . . . . . . . . . . . . 35

4.3 .2 System Inputs . . . . . . . . . . . . . . . . . . . . . . 40

4.4 Derivation of the System Model . . . . . . . . . . . . . . . . 42

4.4.1 Client Behavior Model . . . . . . . . . . . . . . 42

4.4 Routing Algorithms ................ . . 45

4.4 .3 Broker Model . . . . . . . . . . . . . . . . . . . . . . 47

4.4.4 Model for Physical Links and Routers . . . . . . . . . . . 48

4.4.5 Auxiliary Measures . . . . . . . . . . . . . . 48 
4.4.6 Routing Table Size . . . . . . . . . . . . . . . 50

4.4.7 Forwarding Probabilities of Messages . . . . . . . . . . . 54

4.4 Traffic . . . . . . . . . . . . . . . . 68

4.4 .9 Utilization and Delay . . . . . . . . . . . . . 71

4.5 Evaluation ..................... . . . 73

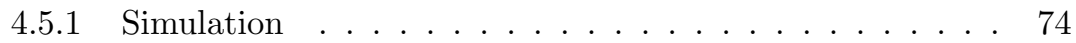

4.5 .2 Insights . . . . . . . . . . . . . . . . . 74

4.5.3 Hierarchical Routing . . . . . . . . . . . . . 79

4.6 Generalizations and Extensions . . . . . . . . . . . . . 88

4.6.1 Multidimensional Notifications/Subscriptions . . . . . . 88

4.6.2 Variable Number of Attributes . . . . . . . . . . . . 93

4.6.3 Utilization and Delay . . . . . . . . . . . . . . 95

4.7 Discussion . . . . . . . . . . . . . . . 96

5 Capacity Planning $\quad 99$

5.1 Introduction . . . . . . . . . . . . . . . . . . . . 99

5.2 Related Work . . . . . . . . . . . . . . . . . . . . 100

5.3 Efficient Calculation of the Model . . . . . . . . . . . . . . . 100

5.3.1 General Workflow for Model Calculation . . . . . . . . . . 101

5.3 .2 Numerical Challenges . . . . . . . . . . . . . . 103

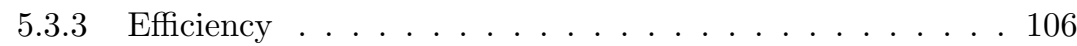

5.4 Optimized Configurations . . . . . . . . . . . . . 107

5.4.1 Optimizing Publish/Subscribe Infrastructures . . . . . . . 107

5.4.2 Optimization Complexity ............... 108

5.5 Case Study . . . . . . . . . . . . . . . . . . . . 110

5.6 Discussion . . . . . . . . . . . . . . . . 115

6 Adaptive Routing $\quad 117$

6.1 Introduction . . . . . . . . . . . . . . . . . . . . . . . . . . . . . . . 118

6.2 Related Work . . . . . . . . . . . . . . . . . . 119

6.3 Hybrid Routing Algorithms . . . . . . . . . . . . . . 120

6.3.1 Rendezvous Broker and Correctness Criterion . . . . . . . 121

6.3.2 Routing Configurations . . . . . . . . . . . . . . . . . 124

6.3.3 Routing Reconfigurations . . . . . . . . . . . . . . . . . 125

6.3.4 Structure of Routing (Re)configurations . . . . . . . . . 127

6.4 Self-optimizing Algorithm . . . . . . . . . . . . . . . . . 130

6.4.1 Optimization Criterion . . . . . . . . . . . 130

6.4 .2 Algorithm Details . . . . . . . . . . . . . . 132

6.5 Evaluation . . . . . . . . . . . . . . . . . 140

6.5.1 Step Response . . . . . . . . . . . . . . . . . 140

6.5.2 Dynamic Situation . . . . . . . . . . . . . . . 143

6.6 Generalizations and Extensions . . . . . . . . . . . . . 144

6.6.1 Adaptive Hierarchical Routing . . . . . . . . . . . . . . 145

6.6.2 Advanced Routing Algorithms and Advertisements . . . . 146

6.6.3 Fault-tolerant Adaptive Routing . . . . . . . . . . . . . . 147

6.6.4 Fault-tolerant Adaptive Hierarchical Routing. . . . . . . . 148

6.6.5 Composition of Self-optimizing Algorithms . . . . . . . . 148

6.7 Discussion . . . . . . . . . . . . . . . . . . 151 
7 Conclusions and Future Work 153

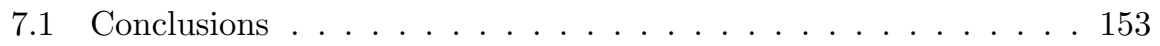

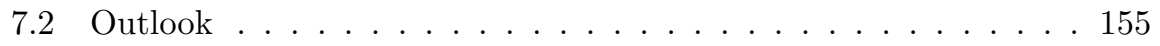

$\begin{array}{lr}\text { Appendix } & 159\end{array}$

A List of Abbreviations and Symbols $\quad 159$

B Routing Configurations of a Binary Tree 163

$\begin{array}{ll}\text { Bibliography } & 165\end{array}$ 


\section{List of Figures}

1.1 Publications containing "publish/subscribe" in the title . . . . . 6

1.2 Topics and organization of this thesis . . . . . . . . . . 8

2.1 Publish/subscribe system model . . . . . . . . . . . . . . . . 12

2.2 Publish/subscribe system architecture . . . . . . . . . . . . . . 13

2.3 Filter model and messages . . . . . . . . . . . . . . . . 15

2.4 Orthogonal dimensions of content-based routing . . . . . . . . . 17

2.5 Advertisement-based routing in publish/subscribe systems . . . . 18

2.6 Hierarchical routing in publish/subscribe systems . . . . . . . . . 19

3.1 Human being and software layers . . . . . . . . . . . . . . 23

3.2 Requirements on publish/subscribe systems . . . . . . . . . . 25

3.3 Optimizations within the life cycle of a publish/subscribe system 26

4.1 General system model . . . . . . . . . . . . . . . . . 35

4.2 Definitions of notification and subscription delay . . . . . . . . 37

4.3 Partially ordered set of delays . . . . . . . . . . . . . . . . . . . . 40

4.4 General modeling approach . . . . . . . . . . . . . . . . . 43

4.5 Markovian chains . . . . . . . . . . . . . . . . . 44

4.6 Queueing model of brokers including clients . . . . . . . . . . . . 48

4.7 Auxiliary measures . . . . . . . . . . . . . . . . . . . . . . . . . . . 49

4.8 Remote routing entries . . . . . . . . . . . . . . 53

4.9 Matching probability for SR/CBR: $1-(1-2 x(1-x))^{k} \ldots$. . 56

4.10 Matching probability for MBR: $1-\left(x^{2 k}+(1-x)^{2 k}\right) \ldots \ldots$. . . 56

4.11 Distribution of notifications . . . . . . . . . . . . . 58

4.12 Notifications probabilities . . . . . . . . . . . . . . 59

4.13 False positive probabilities . . . . . . . . . . . . 59

4.14 Subscription forwarding probabilities for merging-based routing • 64

4.15 Subscription forwarding probabilities . . . . . . . . . . . . . 64

4.16 Advertisements $+\mathrm{SR} \ldots \ldots \ldots 6$

4.17 Advertisements $+\mathrm{CBR} \ldots \ldots \ldots \ldots 6$

4.18 Advertisements + MBR . . . . . . . . . . . . . . 67

4.19 Traffic measures . . . . . . . . . . . . . . . . . . 70

4.20 Remote routing entries . . . . . . . . . . . . . . . . . . . 74

4.21 Qualitative dependencies of measures . . . . . . . . . . . . 75

4.22 Subscription traffic for different routing algorithms . . . . . . . . 77

4.23 Comparison between different routing algorithms . . . . . . . . . 78

4.24 Resulting topologies for 15 brokers $\left(T_{1}, T_{2}, T_{4}, T_{8}\right) \ldots \ldots 1$ 
4.25 Routing table sizes . . . . . . . . . . . . . . . . 82

4.26 Notification traffic . . . . . . . . . . . . . . . . . . 82

4.27 Control traffic . . . . . . . . . . . . . . . . . . . . 83

4.28 Saved traffic compared to FL . . . . . . . . . . . . . . . . 84

4.29 Traffic for $T_{8} \ldots \ldots \ldots \ldots$. . . . . . . . . . . . . 84

4.30 Distribution of broker load . . . . . . . . . . . . . . 85

4.31 Distribution of link load . . . . . . . . . . . . . . . . . . 85

4.32 Maximum expected notification delay (for $\omega=1000 s^{-1}$ ) . . . . 86

4.33 Maximum expected notification delay (for $N^{s}=1000$ ) . . . . . . 86

4.34 Maximum expected subscription delay (for $\omega=1000 s^{-1}$ ) . . . . 87

4.35 Maximum expected subscription delay (for $N^{s}=1000$ ) . . . . . 87

4.36 Matching probability for multiple dimensions . . . . . . . . . . 91

4.37 Probabilities for not covering for multiple dimensions . . . . . . . 93

4.38 Overlapping probabilities for multiple dimensions . . . . . . . . . 94

5.1 Dependencies between quantities . . . . . . . . . . . . . . . 101

5.2 Leaves on different levels . . . . . . . . . . . . . . . . . . . . . . 102

5.3 Error of $q_{k} \ldots \ldots \ldots$. . . . . . . . . . . . . . . . . . . 104

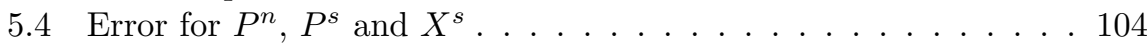

5.5 Runtime: simulation vs. analysis . . . . . . . . . . . . . 106

5.6 Overview on capacity planning . . . . . . . . . . . . . . 108

5.7 Notification delay for different configurations . . . . . . . . . . . 112

5.8 Optimal routing algorithms and load . . . . . . . . . . . . 114

5.9 Optimal routing algorithms and locality . . . . . . . . . . . 114

6.1 Link configuration ... . . . . . . . . . . . . . . 120

6.2 Routing configurations . . . . . . . . . . . . . . . 122

6.3 Rendezvous broker . . . . . . . . . . . . . . . . . . . 124

6.4 Reconfiguration from flooding to simple routing . . . . . . . . . . 126

6.5 (Re)configuration networks . . . . . . . . . . . . . . . 128

6.6 Adaptive routing algorithm . . . . . . . . . . . . . . . 133

6.7 Optimality of adaptive routing . . . . . . . . . . . . . . . 135

6.8 Connection of two overlays . . . . . . . . . . . . . . . 137

6.9 Connecting clients .................... 139

6.10 Step response of the subscription traffic . . . . . . . . . . . . 140

6.11 Step response of notification traffic . . . . . . . . . . . . . . . 141

6.12 Step response of the overall traffic . . . . . . . . . . . . . . . . . 141

6.13 Number of flooding links in a dynamic situation . . . . . . . . . . 143

6.14 Overall traffic in a dynamic situation . . . . . . . . . . . . . . . . 144

6.15 Fault-tolerance for adaptive routing . . . . . . . . . . . . . . 147

6.16 Composition of self-optimizing algorithms . . . . . . . . . . . . . 149

B.1 Routing configurations of a binary tree with three levels . . . . . 164 


\section{List of Tables}

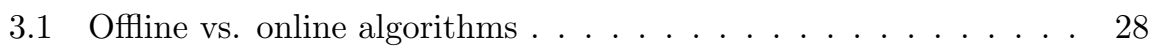

4.1 Comparison of delays . . . . . . . . . . . . . . . . 40

4.2 Comparison of routing algorithm with/without advertisements . 68

4.3 Message type dependencies . . . . . . . . . . . . . . . . 70

4.4 Formulas depending on routing algorithm . . . . . . . 80

$4.5 \quad$ Filter list F List . . . . . . . . . . . . . . . . . . . 88

5.1 Complexity of optimization . . . . . . . . . . . . 110

5.2 Parameters used for topology generation with BRITE . . . . . . 111

6.1 Rendezvous broker $R_{i j} \ldots \ldots \ldots \ldots \ldots \ldots$. . . . . . . . . . . . . . .

6.2 Compatible configurations . . . . . . . . . . . . . . . 138

6.3 Traffics in both scenarios . . . . . . . . . . . . . . 150

A.1 Abbreviations . . . . . . . . . . . . . . . . . 160

A.2 General symbols . . . . . . . . . . . . . . . . . . 160

A.3 Symbols for inputs . . . . . . . . . . . . . . . . . . 161

A.4 Symbols for probabilities . . . . . . . . . . . . . . . 161

A.5 Symbols for outputs including traffic, utilization and delay . . . 162 


\section{Chapter 1}

\section{Introduction}

\subsection{Motivation}

Forty years ago the Arpanet laid the foundation for interconnecting heterogeneous computers in a decentralized manner. From then on, distributed computer systems stepwise pervaded everybody's lives and transformed the world into a virtually small village. Trends like the availability of smart phones, falling prices for all types of hardware and increased program usability for users even reinforce this trend. Soon the "Internet of things" ${ }^{1}$ may become real as a vision, where everyday's objects are interconnected and can be reached by their individual IPv6 address [30]. This highly-connected world opened the way for myriads of distributed applications for end-users and professionals, too. For end-users, the most present are collaborative platforms, like Wikipedia and social networks, like Facebook, just to name a few. For enterprises, XaaS ${ }^{2}$, cloud computing and smart grids became a major part of their business.

Nevertheless, large-scale distributed applications often suffer from the scalability problem meaning that an increasing number of users does lead to an over linear increase of the required system resources, like bandwidth or computing power. This problem often stems from the way information are disseminated from information producers to information consumers. In modern applications, this problem becomes even worse since not only the number of consumers but also the number of information producers rapidly increases. Each human being tends to be a sender, by providing the status in a social network, writing blog entries and so forth. Furthermore, all network connected objects, like sensors, act as producers by providing measured data, like the current temperature and noise values or RFID-tags information. This vast of producers of information on the one hand and the wish of the consumers to filter out interesting information on the other hand drives the classical distributed programming paradigm based on the request/reply approach to its limit. The request/reply model may be compared with the email system where users periodically poll for new emails but most polls are empty since no mail has be delivered during the last polling interval. When many clients use a high polling frequency, an overload of the server could be the consequence. This could further increase the polling fre-

\footnotetext{
${ }^{1}$ http://en.wikipedia.org/wiki/Internet_of_Things (last visit: 2012-06-04)

${ }^{2}$ With instances, such as Software as a Service (SaaS) etc.
} 
quency due to impatient clients. This problem can be solved by introducing a push mechanism which proactively delivers new emails to the receiver.

In general, the advantages of these event-based systems, such as bandwidth reduction and responsiveness, are often only qualitatively enumerated. However, in a real world application scenario there are more concrete demands. On the one hand, designers need to quantitatively predict the performance of such systems based on exactly defined performance metrics. Thus, designers are able to configure the system or to perform a capacity planning. On the other hand, operators of such systems need a support for runtime optimizations to have automatic adaption on changing environments. This thesis provides answers to both previously mentioned challenges by introducing a new holistic view on modeling and optimization of distributed event-based systems.

\section{Distributed Event-Based Systems}

The central term and heart of the matter of Distributed Event-Based Systems $(D E B S)$ is event. An event in computer science can be defined in the style of the word event in natural language. It describes "Anything that happens, or is contemplated as happening." [61] Often an event is related to a state change like the start of an airplane, the affection of a colleague or the occurrence of an earthquake. More formally, in computer science an event is a piece of information that an event source pushes or publishes at a certain time into an event-based system. The trigger for this publish operation either lays outside the event-based system, like e.g., the computer hardware and the natural environment, or it is generated within the system by any arbitrary processing of other events. This way two types of events can be distinguished, simple or raw events and complex or composite events. With a blackbox view on an event source it may be impossible to determine if it generates simple or complex events, because they are structurally identical.

Recalling the above examples for events one may disagree with defining the "start of an airplane" as an event, because he/she may not be interested in that or may not have the possibility to detect it. This natural definition symbolizes another important aspect of an event-based system which is event filtering. In this example, the person either consciously or unconsciously filters the events that he/she is interested in to reduce the amount of information to process. Translating this idea to computer science, an event-based system provides on the one hand the possibility to produce events and on the other hand to subscribe for events by defining filters in a filter language. The mentioned subscribe operation is linked to a callback initiated by the event-based system to inform the subscriber about interesting events. Beside event publishing and subscribing the term $D E B S$ also emphasizes the very nature of many modern applications which is its spatial distribution. Thereby, we follow the rather old definition of general distributed systems of Bal [4]: "A distributed computing system consists of multiple autonomous processors that do not share primary memory, but cooperate by sending messages over a communication network."

In fact, event-based systems have been used almost since the beginning of the computer age. The concept of hardware interrupts [100] from the 1970s falls within this scope. It has been introduced to avoid wasting the processor time in polling loops waiting for external signal changes. Instead asynchronous signals indicate the need for attention. Later the concept of hardware interrupts 
has been extended to software interrupts implemented as special instructions, which cause a context switch to an interrupt handler similar to a hardware interrupt. Later, event-based systems became an autonomous branch of software architecture. First steps were architectures for non-distributed softwares like the JAVA SwING framework for GUIs and event-condition-action rules [77] for active databases, or design patterns like the observer pattern. With the ascent of local area networks and the Internet, the focus shifted towards the distributed nature. Challenges of the distribution were solved by introducing middleware solutions, such as JAVA RMI, hiding all network and distribution related issues such as faults, replication, etc. The first middleware implementations focused primarily on a request/reply communication scheme as an analogy to the wellknown imperative programming for non-distributed software. Later more and more extensions and approaches that also considered the event-based communication for distributed systems were developed. Some of these developments became popular standards such as the Corba Event and Notification SeRvice, Java Message Service (JMS), and the Data Distribution Service FOR REAL-Time Systems (DDS). Nowadays, many large-scale distributed systems are at least internally DEBS. For example in FACEBOOK, probably the biggest distributed application, every minute "... users submit around 650,000 comments that need to get routed to the correct viewers" ${ }^{3}$. This is internally realized by events. This event interface is also provided by the realtime API for applications programmers ${ }^{4}$. Facebook is only one example of the big amount of other distributed event-based applications like computational/targeted advertising, intrusion detection, real-time data analysis including fraud detection.

A recent development step in DEBS are event-driven architectures (EDA) for big enterprises, which extend existing service-oriented architectures (SOA) to event-driven SOA. After a long time of having synchronous request/reply based service compositions within a SOA, system developers and users suffered from its limited flexibility and scalability. Therefore, the event-based systems' approach was taken and adapted to business requirements. Beside improved flexibility and scalability, the main advantage for companies is to speed-up their processes. Instead of have daily or hourly batch-jobs, reactions are now done instantaneously, as it is described in promotional terms like zero latency enterprise (ZLE) or real-time business [95]. In connection with this development, also the term complex event processing (CEP) [60] has been established with a special focus on the fast detection of complex events within a huge "event cloud". This event cloud is generated by myriads of information, expressed by events, flowing into a company. Event sources include external events, like news feed, customer contacts, business-to-business communication, as well as internal events from all relevant business processes and IT events. For CEP, users are allowed to express their complex events by special languages like CQL which is an extension to the request of old data by SQL. Furthermore, there are approaches to define complex events by rules. A special application of a CEP engine is computational finance, also called algorithmic trading resp. high-frequency trading systems (HFT) which nowadays do handle $90 \%$ of all trading activities at stock exchanges.

The mentioned development of DEBS was driven by its advantages over es-

\footnotetext{
${ }^{3}$ http:/ facebook.com/notes/facebook-engineering/live-commenting-behind-the-scenes/ 496077348919 (last visit: 2012-06-04)

${ }^{4}$ https://developers.facebook.com/docs/api/realtime/ (last visit: 2012-06-04)
} 
tablished technologies. Using an event-based system inverts the control of the well-known request/reply scheme. Now, components are more anonymous, decoupled and become only active when new data is available. When events are pushed into the system, the sender does not address the receiver and, therefore, does also not know about the number of receivers. This way, senders and receivers may be added dynamically to the system, leading to more flexibility and scalability of the system and the application. The avoidance of permanent requests for detecting information updates also reduces the latency as well as the load of the system. Furthermore, the distributed realization of an eventbased system allows local filtering and load balancing which further increases the scalability but requires new approaches in design and management.

Despite these advantages there are also drawbacks in the event-based approach. Specially, the performance of DEBS degrades significantly at very high event rates if not filtered before entering the system. Such situations may completely stall the system and hinders it to process relevant events if not prioritized. This is one reason why DEBS must be provisioned so that load does no exceed the capacity.

\section{Design and Management of Publish/Subscribe Systems}

Beside a database or a tuple space, a publish/subscribe middleware is a common choice for the implementation of distributed event-based systems because it decouples clients in time, space, and program flow [31]. This way, the design of distributed applications is simplified and the system in general benefits from the before mentioned advantages, such as flexibility, scalability and responsiveness.

Nevertheless, a publish/subscribe middleware as a communication platform requires new approaches for its design and management. The design of a publish/subscribe system mainly deals with the challenge how to determine the required resources, e.g., the number of hosts, RAM and bandwidth, as well as the configuration and parameters of the middleware. The result of the design process heavily depends on the definition of a performance or a Quality-ofService (QoS) level. On the other hand, the management of publish/subscribe systems is focused on runtime issues which comprise a wide variety of tasks including the reconfigurations of the broker overlay network, congestion control and the handling of errors. Generally, there are three main factors that drive the development and improvement of techniques for the design and management of a publish/subscribe middleware.

Complexity and System Size. Complexity is a central challenge of mankind. On the one hand, it is simply caused by the growing number of system components and data. For example, the Facebook backend serves over 100 million pieces of content that may receive comments every minute generated by 600 million users. The trend to a growing system size is reinforced by the trend of building systems of systems. On the other hand, the unpredictable behavior of system elements and dependencies among them do further increase the complexity. Especially, if the analogous world including the human being is involved in the system, new feedback loops are introduced with unclear consequences of the behavior. 
Dynamic Environments. The dynamic environments of a modern computer system are the second big challenge beside the complexity. In the past, most systems were deployed in a rather static environment with restricted access for users. Nowadays, many systems are connected via the Internet which makes them available for a wide range of users. Furthermore, the growing popularity of mobile devices and availability of low-price data flatrates again widens the access of user to such systems. User are now completely decoupled from their geoposition. This still ongoing process will move the worlds dynamic behavior, which is described by social, economic, cultural subsystems, etc. into computer systems.

Growing Costs. Caused by the tremendous development of all hardware components of modern computer systems described by Moores law, an engineer can build systems of considerable scale at acceptable hardware costs. In opposite, running and maintaining large-scale software systems is an expensive task because it often requires large numbers of well-trained personnel. This is actually a consequence of the complexity and the dynamic environments. This way, the costs are a mapping of the complexity and dynamics to an economic measure.

Taking these factors into account, it seems that sooner or later the design and management of such complex systems by human designers and operators is reaching its limits. From a certain point, humans are not able anymore to draw correct design and management decisions because the consequences of their actions are unforeseeable. To tackle this problem, over the last years research on automated management has become an increasing interest in academia [70] and industry [49]. Furthermore, design tools are developed to optimally build and deploy such systems.

Enriching systems with automated management features requires to establish "knobs" to allow a reconfiguration without service interruption. Furthermore, an intelligent decision criterion has to be established which pushes the system towards the required direction. A distributed algorithm for a single knob is called adaptive algorithm or, if referred to an optimization, a self-optimizing algorithm. Main classes of adaptive algorithms include fault management and performance optimizations. Enriching systems with adaptive algorithms enables them to automatically adapt to dynamic environments while reducing the amount of human intervention. This way, the administration costs are reduced and designers are enabled to build bigger systems.

Despite this runtime management, the designer also needs to determine an initial configuration of the system which is important if the system is put into production. This a priori design is important to avoid congestion at system start which is not rarely observed at launches of websites, and, to avoid over provision of the system while wasting resources. Both aspects are again directly related to costs.

\subsection{Shortcomings of Current Approaches}

In this thesis, we present the results of our research on modeling and optimizing content-based publish/subscribe systems. The research community in this area 


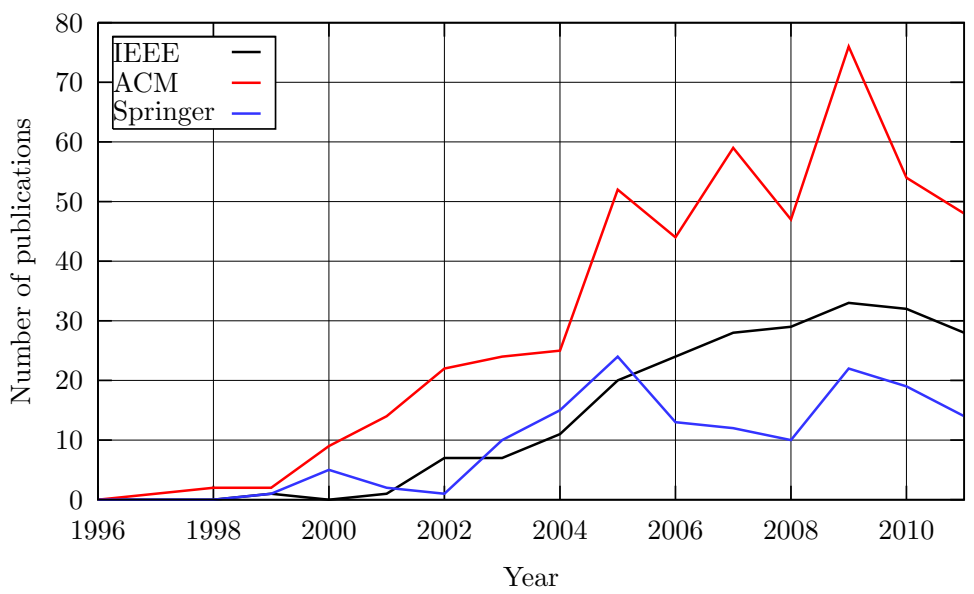

Figure 1.1: Publications containing "publish/subscribe" in the title

has been very active over several years as depicted in Figure 1.1, although the number of publications seems to have already passed its climax. However, there are still shortcomings in fields of system models and optimization strategies of publish/subscribe systems listed in the following.

No Holistic View. When applying the well-known divide-and-conquer method to solve complicated problems, the holistic view on the entire lifecyle of systems tends to fade from the spotlight. Since algorithms are not designed to work together they finally cannot be composed. For example, there are often different assumptions about the structure of the network, about clients of the system or about faults. Therefore, single algorithms cannot be run simultaneously and the design result does not consider all "knobs" of the system. In real scenarios, where a company wants to design and manage a publish/subscribe system it needs a model that is used for both the design and the management. This can only be reached by a holistic view.

Lack of Appropriate System Models. It is common practice to evaluate publish/subscribe systems by simulation. However, there is no standard simulation environment available up to now and the impact of parameter variations on the system behavior is often unclear. Analytical models can serve to create a deeper understanding of publish/subscribe systems and relieve researchers from expensive simulations. Although the research community provided some models, many of them suffer from their limitations due to rather strict assumptions and they often cannot be extended or adapted to individual requirements.

Lack of a Capacity Planning Strategy. The realization of a capacity planning strategy of a system based on simulations suffers from high hardware requirements. Even bigger cluster computers come to a limit if a system is extensively studied. Therefore, such provisioning tasks are often done manually based on trial and error or experience. A capacity planning strategy that is based on a system model, the requirements analysis and the estimation resp. 
measurement of environmental parameters avoids resource-intensive simulations and, therefore, makes it easy to find optimized solutions.

Only Manual Adaptation. Computer systems become bigger and environments become more dynamic and unpredictable. Both in Internet-scale and in small scale networks, it becomes evident that a manual adaption is hard to realize or even impossible. In publish/subscribe research the routing algorithm is often assumed to be configured at design time or at the deployment. Mostly, it is set without any explanation. But even an a priori analysis of the system which outputs the optimal algorithm leads to suboptimal performance if the environment, i.e., the client behavior, changes. Furthermore, routing algorithms are assumed to be homogeneous in the sense that there is only one algorithm within the whole network.

\subsection{Contributions of this Thesis}

This thesis adds contributions for different parts of the life-cycle of a publish/ subscribe system. The focus is on both offline optimizations including a capacity planning strategy based on a stochastic system model, and online optimizations, containing adaptive routing based on hybrid routing schemes and its composition with other algorithms. In particular, we have four contributions which are detailed in the following.

Stochastic System Model. A stochastic system model of a publish/subscribe system is the key to a deeper understanding of these systems. In publish/ subscribe research, most models are still simple and do not cover major aspects of real systems. Therefore, many researcher use simulation studies which are time consuming and often hard to compare. In this thesis, we derive a stochastic system model based on probability and queueing theory that includes all major inputs, like physical network and client behavior. The model has a modular design leading to a general framework that is easy to extend and to adapt to a wide range of real-world systems. The model allows to determine common performance measures to predict the QoS of the publish/subscribe system and is, therefore, an important foundation for provisioning.

Capacity Planning. Meeting predefined QoS requirements with a minimal amount of resources is a challenge for all system designers and operators. Due to the lack of good models of publish/subscribe systems, capacity planning was not possible for a long time. In this thesis, we show how the introduced stochastic system model can be used for provisioning a publish/subscribe system and how the capacity planning can be integrated into the life cycle of the system. Furthermore, we explain how the model may be used to reach individual QoS goals based on measured or estimated environmental data. Thereby, our special focus is on the efficiency of the determination of optimized configurations.

Hybrid Routing Schemes. In the publish/subscribe community, lots of routing algorithms have been suggested yet. While the simplest one just floods notifications, advanced algorithms analyze filter similarities to reduce the control 


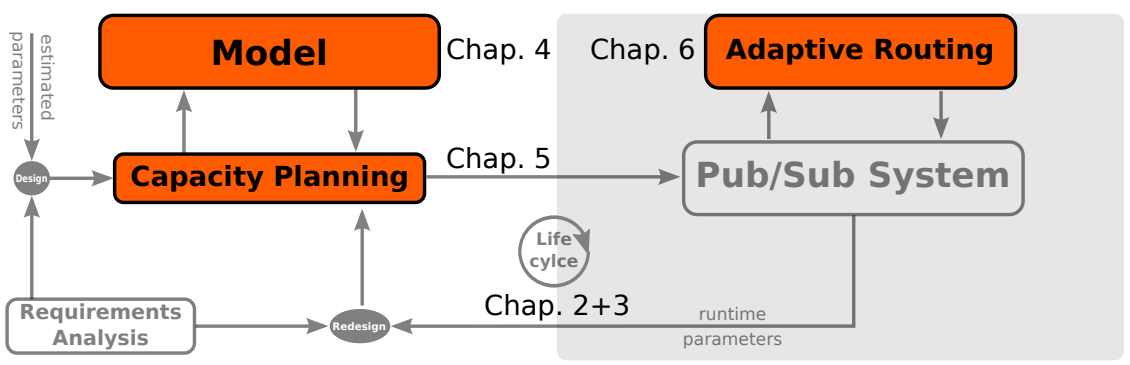

Figure 1.2: Topics and organization of this thesis

message overhead. With this thesis we add the possibility to combine different routing algorithms within one overlay network without violating the correctness of the publish/subscribe system. This hybrid routing allows to get the best from different routing algorithms. Furthermore, by making hybrid routing reconfigurable we provide a new "knob" for a self-optimizing algorithm.

Adaptive Routing. The routing algorithm plays an important role for the efficiency of the publish/subscribe infrastructure. Each single algorithm is optimal for certain network situations defined by the publishing and subscribing behavior of the clients. Since nowadays systems tend to be used in dynamic environments it is desirable to adapt the routing algorithm according to the network load situation. For this reason, we introduce the adaptive routing which is a decentralized self-optimizing algorithm for publish/subscribe systems. It is based on a local reconfiguration criterion and generates only a minimal amount of coordination effort and computational overhead.

\subsection{Organization of this Thesis}

This thesis starts with an introduction into publish/subscribe in Chapter 2. Then, in Chapter 3 we explain optimization approaches within the life cycle of a publish/subscribe system. This chapter is the central theme of all following contributions of the thesis. The related topics and the organization of this thesis embedded into the lifecylce of a publish/subscribe system are depicted in Figure 1.2.

In Chapter 4, we lay the foundation for the offline capacity planning of publish/subscribe systems by introducing a new stochastic system model. After identifying required input and output parameters, we stepwise derive a set of formulas that allow us to predict all major performance measures of a publish/ subscribe system, namely, traffic and utilization of brokers and links as well as delay of different messages types and routing tables sizes.

In Chapter 5, we use the derived stochastic system model for the capacity planning, i.e., the provisioning of a publish/subscribe infrastructure. We present an approach for the efficient calculation of the model and evaluate the results in a case study.

Chapter 6 deals with the online self-optimization of the routing algorithm. First, a new type of routing algorithms is introduced that combines different 
already known algorithm within one network. Based on this new scheme, a selfoptimizing strategy is presented which can be used to improve the performance of the publish/subscribe middleware. The suggested algorithm is evaluated in different scenarios. Another topic in this chapter is the composition of different self-optimizing algorithms including fault-tolerance.

With Chapter 7, we conclude this thesis and summarize the results. Furthermore, we discuss problems which remain open and sketch areas for future work. 


\title{
Chapter 2
}

\section{Distributed Content-based Publish/Subscribe Systems}

\author{
Contents \\ 2.1 Introduction . . . . . . . . . . . . . . 11

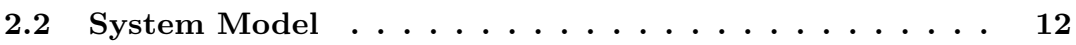 \\ 2.3 System Architecture .............................. 12 \\ 2.4 Matching and Routing ............... 14 \\ 2.4 .1 Data and Filter Model . . . . . . . . . . . . . . . . 14 \\ 2.4 .2 Correctness . . . . . . . . . . . . . . . 15 \\ 2.4.3 Matching and Forwarding . . . . . . . . . . . . . . . . 16 \\ 2.4 .4 Notification Routing . . . . . . . . . . . . . . . . . . 16 \\ 2.5 Basic Assumptions . . . . . . . . . . . . . . . 19
}

\section{$2.1 \quad$ Introduction}

In this chapter, we lay the foundations for this thesis. We start with Section 2.2, where we introduce the system model which is the blackbox view on a publish/ subscribe systems. In this thesis we realize the system in a distributed brokerbased way whose architecture we describe in Section 2.3. For this broker-based architecture, routing and matching are central issues. Thus, in Section 2.4 we give a short introduction to selected algorithms. The presented algorithms cover all major algorithms that are used in academic publish/subscribe middlewares such as PADREs [32], REBECA [73], HeRmes [79] and commercial middlewares such as GRYPHON [99]. We end up this chapter with Section 2.5, where we provide basic assumptions the thesis is built on. 


\subsection{System Model}

A publish/subscribe system consists of a notification service and a set of clients that interact via this service. Clients can interact with the notification service as publishers (a.k.a. producers) or subscribers (a.k.a. consumers). While publishers publish notifications $(n)$ containing information about events, subscribers issue subscriptions $(s)$ that contain filters $(F)$ describing the interest of the subscriber which is a subset of all notifications. On the implementation level, notifications and subscriptions may contain more information like additional meta-data, for example. The notification service is responsible for bringing publishers and subscribers together by delivering published notifications to those clients which issued a subscription with a filter that matches these notifications. For the delivery, the notification service usually calls a callback function which has been provided by the client when subscribing. This publish/subscribe system model has two notable properties. First, despite to "channel-based" schemes like IP multicast [20], the addressing is based on the content of messages (notifications). This enables an anonymous and flexible $n: m$ communication. Second, as a mediator the notification service decouples clients in space, program flow and, depending on the implementation, also in time [31].

The basic interface of the notification service consists of the following operations which can be used by the clients: $\operatorname{pub}(n)$ for publishing a notification $n, \operatorname{sub}(F)$ and unsub $(F)$ for (un)subscribing for a subscription $F$. Additionally, the operation notify $(n)$ is called on a client by the notification service in order to inform it about a new notification $n$ it subscribed for. Advanced publish/subscribe interfaces also contain the operations $\operatorname{adv}(F)$ and $\operatorname{unadv}(F)$ allowing publishers to (un)advertise future publications described by filter $F$. These advertisements are used to optimize algorithms inside the notification service. Figure 2.1 summarizes all described operations. The direction of the arrows indicates the direction of message resp. information flow. The meaning of the depicted broker layer is explained in the following section.

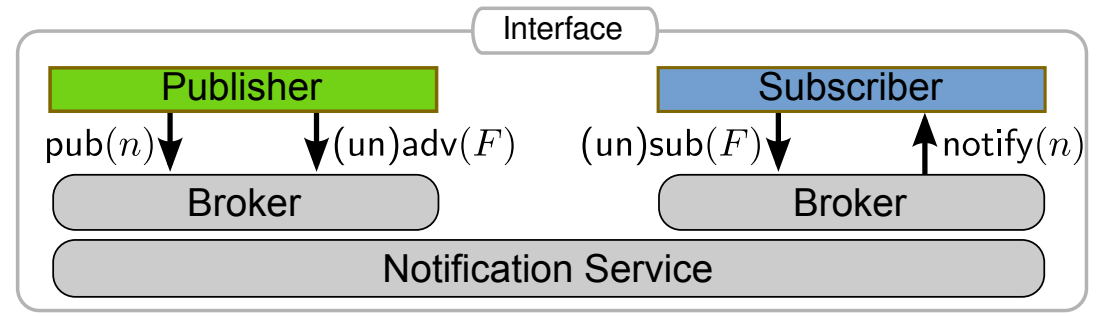

Figure 2.1: Publish/subscribe system model

\subsection{System Architecture}

Instead of layering the notification on top of a peer-to-peer substrate [101], in this thesis we focus on a distributed implementation of the notification service consisting of a set of brokers $\mathcal{B}$ forming a broker overlay network with a topology $T$. An overlay link between two broker $B_{i}$ and $B_{j}$ is indicated as $B_{i} B_{j}$. We require an acyclic topology with FIFO links which is a prerequisite for using 


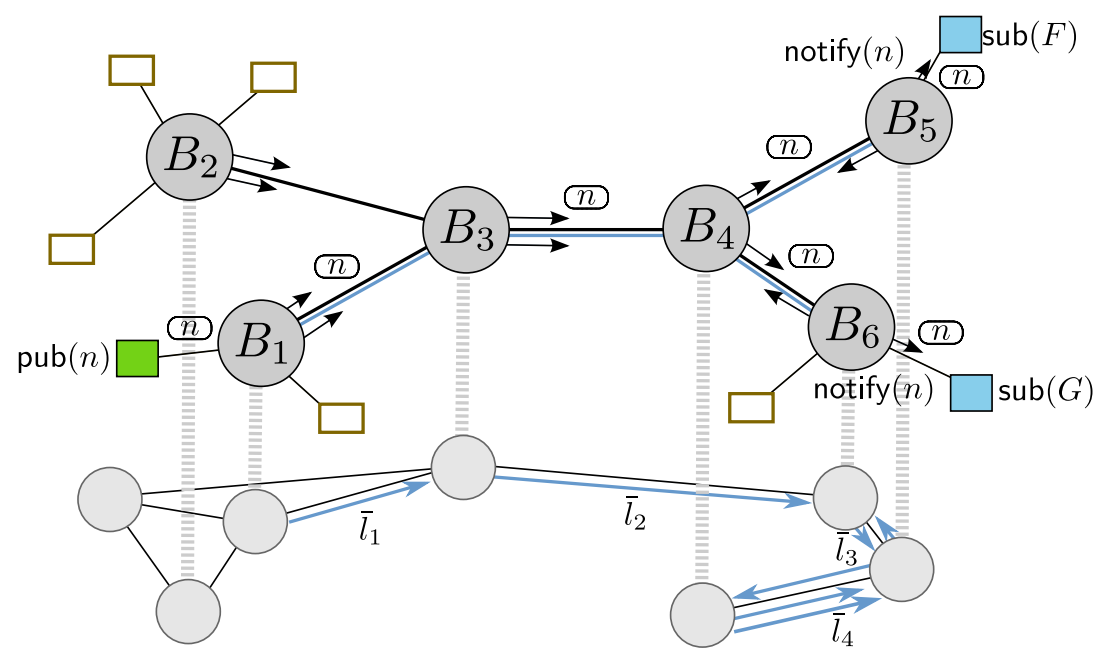

Figure 2.2: Publish/subscribe system architecture

advanced routing algorithms such as covering-based routing. This is common in literature because it simplifies maintaining message ordering and preventing message duplicates. Furthermore, in opposite to peer-to-peer substrates we have full control over the topology of the overlay and message flows, and, thus, are able to optimize the event dissemination in our means.

Exchanged messages between brokers in such an overlay network are notifications $n$ and control messages such as subscriptions $s$ and advertisements $a$ which are used to update status information (cf. Section 2.4). Unsubscriptions and unadvertisements that are also control messages we mark with a bar, i.e., $\bar{s}$ resp. $\bar{a}$. Each broker provides the interfaces described in the last section and acts as a proxy of the notification service, i.e., by connecting to an arbitrary broker clients have access to the complete distributed publish/subscribe system. Figure 2.2 illustrates the architecture of a distributed publish/subscribe system we refer to in this thesis. Each broker $B$ maintains a routing table $R T_{B}$ in order to decide whether and if where to forward an incoming notification to. A routing table holds routing entries which are $\langle\mathrm{F}, \mathrm{D}\rangle$ pairs, where a destination $D$ is either a neighbor broker or a local client. In Figure 2.2, the routing table entries are depicted as black arrows. We differentiate between local and remote routing entries. In the figure, all routing entries are remote except for the ones at brokers $B_{5}$ and $B_{6}$ with local clients. $N_{B}$ is the set of remote destinations of $B$ and $L_{B}$ is the set of local destinations of $B$. In the example figure this is, e.g., $N_{B_{4}}=\left\{B_{5}, B_{6}\right\}$ and $L_{B_{5}}=\{S\}$. Later in this thesis we often ignore the local entries when talking about the routing table since the number of local entries is equal for all algorithms. Despite the common definition of a routing table $R T_{B}$, we additionally introduce the term filter list $F \operatorname{List}(B, D)$ of a broker $B$ pointing to destination $D$. It contains all filters of routing table entries of $R T_{B}$ with destination $D$. In Figure 2.2 this is, e.g., $F \operatorname{List}\left(B_{2}, B_{3}\right)=\{F, G\}$. Obviously, the set union of all filter lists of $B$ and its destinations is equivalent to $R T_{B}$.

The broker overlay runs on top of a physical infrastructure, also called physical network or underlay. It consists of routers and links. For both physical com- 
ponents we use the symbol $\bar{l}$. On the upper level, messages traverse the broker overlay topology based on their content and the currently active subscriptions. On the lower level, messages are routed through the physical network between neighboring brokers in the overlay topology. The overlay network is a logical network whose nodes are the brokers and whose links are paths in the physical network. When a broker sends a message to another broker over an overlay link, the message is injected into the physical network and forwarded stepwise over physical links and routers to the receiving broker. Therefore, overlay links sharing physical links or routers also share their physical bandwidth. With bandwidth we mean the available data rate as it is normally used in computer networking. In Figure 2.2 one can see that this may not be optimal if physical links are traversed multiple times. In the figure the mapping is depicted in gray. While for example messages that pass overlay link $B_{1} B_{3}$ have to traverse a single link $\bar{l}_{1}$, overlay link $B_{4} B_{6}$ consists of $\bar{l}_{3}$ and $\bar{l}_{4}$. There are also underlay links that share several overlay links such as $\bar{l}_{3}$.

\subsection{Matching and Routing}

Matching and routing are central parts of a distributed publish/subscribe system. Before explaining them, we first discuss the data and filter model and explain what a correct publish/subscribe system is.

\subsubsection{Data and Filter Model}

The data and filter model is a main property of each publish/subscribe system. These models determine the expressiveness and the complexity of matching and routing algorithms. From the programmers view, the expressiveness is an important decision criterion for or against an arbitrary middleware. The more powerful the data and filter models are, the easier applications can be developed. For the middleware designer a more expressive model increases the complexity, and, thus, restricts internal middleware optimizations.

While the data model refers to the data representation of events, the filter model describes which types for filters can be used to express interests. Both models are closely linked. For example, publish/subscribe systems that use a $X M L$ description of events [58] often use XPath filters. Other pairs are tuples/templates similar to Linda [35], event objects/filter objects and, very often used, name-value pairs/conjunctive attribute filters.

In the latter model, the content of a notifications is represented as a set of attributes where each attribute is a name/value pair. For example, the notification containing three attributes $\{($ id, nobody $),($ status, ill $),($ time $, 6: 10 a m)\}$, can be used to indicate that in a social network the status of the member nobody was set to "ill" at $6: 10 \mathrm{am}$. In this thesis, we abstract from the semantics of notifications [104] and do not cover aspects like event hierarchies or scopes [33]. As already mentioned, filters are normally defined as conjunctions of attribute filters, where each attribute filter places a constraint on the value of a given attribute. For example, the filter $\{$ name $=$ nobody, time $<1 p m\}$ matches the above notification. We implicitly assume that attributes of the notification, operators and operand are compatible. 


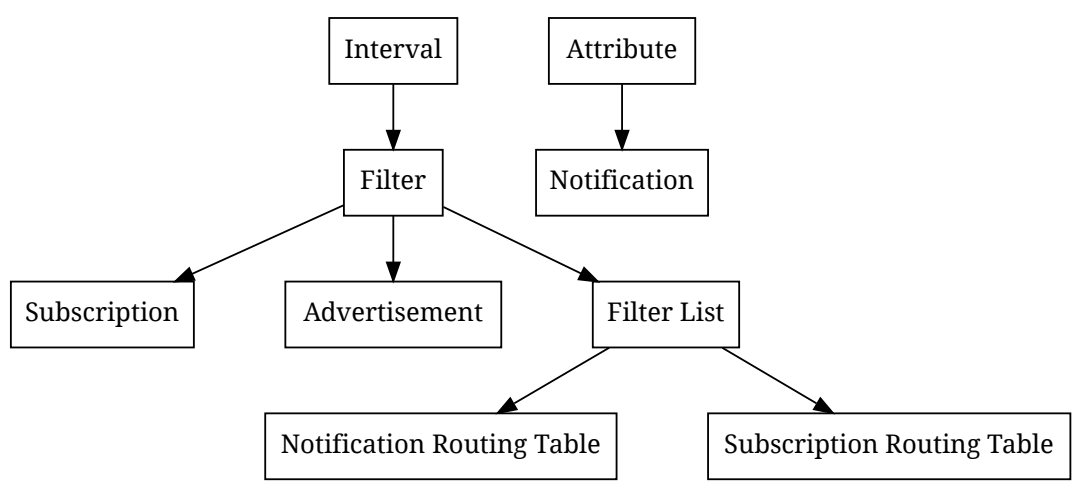

Figure 2.3: Filter model and messages

For our later analysis in this thesis we restrict this general data and filter model as follows. First, we model each notification $n$ by a list of $d$ numbered attributes $x_{i}$. Therefore, we may represent $n$ as a $d$-dimensional vector $n=\left[x_{1}, \ldots, x_{d}\right]$. Assuming real values for $x_{i}$, a notification is a dot in the $d$-dimensional space $\mathbb{R}^{d}$. On the other hand, a filter $F$ consists of $d$ attribute filters each restricting one dimension. Such an attribute filter is an interval $I_{i}=\left[a_{i}, b_{i}\right]$ with two numeric bounds $a_{i}$ and $b_{i}$. Since for $a_{i}$ and $b_{i}$ we also allow values " $\pm \infty$ ", filters are able to represent operators like " $\leq$ " resp. " $\geq$ ". A filter is a logical conjunction of its $d$ attribute filters $F=I_{1} \wedge \ldots \wedge I_{d}$ which defines a compact subspace of $\mathbb{R}^{d}$, a multidimensional interval. The filter can be represented by a $d$-dimensional vector with numeric 2 -tuples as its elements $F=\left[\left[a_{1}, b_{1}\right], \ldots,\left[a_{d}, b_{d}\right]\right]$. These filter can either be part of a subscription or of an advertisement. The relationships between all mentioned elements are shown in Figure 2.3.

\subsubsection{Correctness}

Since publish/subscribe middlewares are more and more used in system-critical or financial areas they must provide their functionality in a dependable way. To have a strict definition of a proper functioning publish/subscribe system the correctness is often used. Following Lamport [56], the correctness of a distributed system is described by the properties safety and lifeness While safety in this sense means that "something irremediably bad" will never happen, liveness describes that "something good" will eventually happen. Often the additional property fairness is required avoiding the discrimination of system users. This approach has been applied to publish/subscribe by Mühl et al. [66]. With the help of temporal logic the correctness of a publish/subscribe system is expressed as follows:

- Safety. Each subscriber must not receive a message it has not subscribed to. Furthermore, each notification is delivered at most once.

- Lifeness. It is guaranteed that after a finite time after a client has issued a subscription all matching notifications are delivered. 
- FIFO-Publisher-Order. Notification are delivered to interested clients in the same order as they have been published by the publisher.

This definition is a rather strong requirement on publish/subscribe systems because no loss, duplication or disordering is tolerated. Nevertheless, a middleware that guarantees the correctness heavily simplifies the programming of distributed event-based applications, because the complexity is shifted from the programmer to the middleware layer. Later in this thesis we often refer on this definition.

\subsubsection{Matching and Forwarding}

When a broker $B$ receives a notification $n$, it decides based on its routing entries whether to forward it to a destination or not. Therefore, $B$ checks whether a filter (for a destination) matches $n$. This is often expressed by a binary function $F(n)=\{$ true, false $\}$. The problem of determining all filters that match a notification is termed the matching problem. With channel-based matching, each notification is tagged with a selected channel and subscribers can only subscribe to channels. Subject-based matching is more expressive, because notifications are published with respect to a selected subject, where the subjects themselves can be arranged in a hierarchical fashion in contrast to "flat" channels. For this thesis we mainly use content-based matching based on the data and filter model presented in a previous section. Many different algorithms have been proposed in the past for content-based matching. Beside simple brute force algorithms, the most notably are the counting algorithm [105], decision trees [1], and binary decision diagrams [18]. With the advent of GPU computing these rather old algorithms returned to the focus [63]. For this thesis these algorithms are not considered.

Mühl et al. distinguish between matching and forwarding [66]. While matching refers to the problem of determining all filters in a routing table which match a given notification, the forwarding problem consists of determining all destinations a given notification must be sent to. The difference between both problems is that for notification forwarding it may not be necessary to find all matching filters in a routing table $R T_{B}$ for a given notification. Instead it is enough to find at least one matching filter in the related filter list $F \operatorname{List}(B, D)$. Only in the worst case, all filters have to be checked to find out that the notification does not have to be forwarded.

\subsubsection{Notification Routing}

While matching and forwarding are tasks on single brokers, the notification routing is a class of distributed algorithms that deal with the efficient routing of notifications from publishers to interested subscribers through the broker network. In literature, there are three major dimensions in which content-based routing algorithms have been developed. These orthogonal dimensions are shown in Figure 2.4. Each algorithm exploits the matching algorithms of the previous section. The requirement on each algorithm is to guarantee the correctness of the entire publish/subscribe system as described in Definition 2.4.2. Thus, it must avoid false negative deliveries of notifications and should avoid false positives $(F P)$. Following, we describe all three dimensions of content-based routing. 


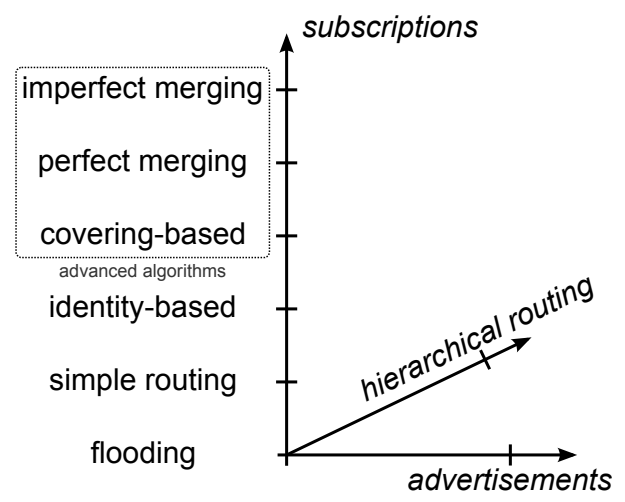

Figure 2.4: Orthogonal dimensions of content-based routing

Subscription-based Routing Algorithms. To forward published notifications to interested subscribers, all routing algorithms maintain routing tables. The simplest routing algorithm is flooding (FL), where every notification is sent stepwise to all brokers in the system. Therefore, each broker forwards an incoming notification to all its connected neighbors despite to the sender of it. Furthermore, the incoming notifications are matched against its routing table which only contains local subscriptions of local clients. Obviously, this routing strategy is suboptimal, when there are lots of brokers with local clients that are not interested in most of the published notifications.

Another approach is simple routing (SR), where subscriptions instead of notifications are flooded in the overlay network. On receiving a subscription, the receiving broker installs a routing entry in its routing table pointing to the broker from which it received the subscription. This algorithm generates eventually global knowledge about filters as it is shown in Figure 2.5(a). Unsubscriptions are forwarded like subscriptions but lead to the removal of the previously installed routing entry. Incoming notification are matched against the routing table to decide where to forward them (cf. forwarding problem of the previous section).

In opposite to simple routing, which treats each subscription in isolation, advanced routing algorithms try to exploit similarities between newly issued filters and filters stored in routing table. In the case of identity-based routing (IBR), subscriptions are not forwarded to a neighbor broker if an identical subscription has already been sent there in the past. Two subscriptions with filters $F_{1}$ and $F_{2}$ are identical if the set of notifications both match are identical.

With covering-based routing (CBR), subscriptions are tested with respect to their covering relation. A filter $F_{1}$ covers a filter $F_{2}$ if the set of notifications it matches is a superset of the set of notifications $F_{2}$ matches. In this case, a subscription $s$ does not need to be forwarded to a neighbor broker. Coveringbased routing requires additional effort to determine the covering relation, and, a more sophisticated unsubscription handling. If a covering filter is removed, covered subscriptions that have not been forwarded so far have to be reissued to the neighbor before the covering filter is removed.

With merging-based routing (MBR), brokers merge filters in order to create a new filter. This new filter either perfectly matches the set of notifications 


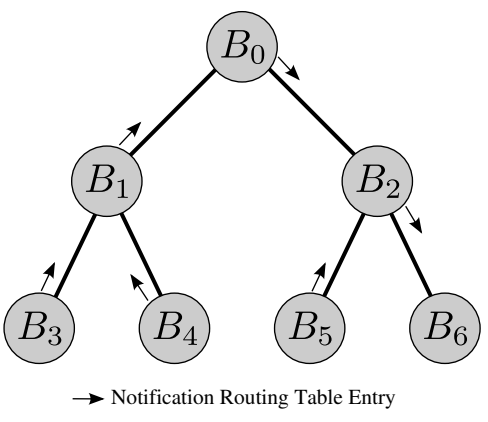

(a) Standard routing

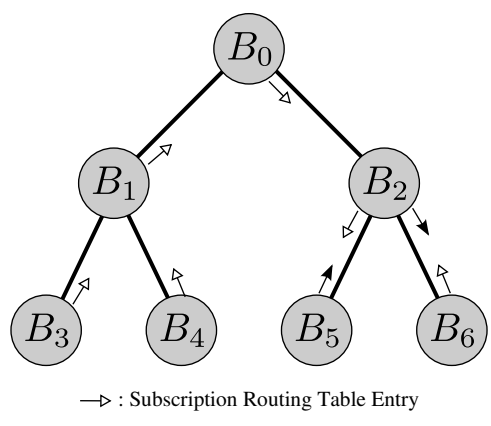

(b) Advertisement-based routing

Figure 2.5: Advertisement-based routing in publish/subscribe systems

covered by the constituent filters (perfect merging) or it covers a superset of those notifications (imperfect merging). The new filter that results from merging can accordingly be used to perform subscription forwarding optimizations similar to covering-based routing.

Following the differentiation between perfect and imperfect merging, we define perfect routing algorithms as those algorithms that do not generalize, i.e. that the resulting filter which is forwarded does not properly covers the union set of all active filters. Flooding as well as imperfect merging-based routing are both not perfect algorithms.

Advertisement-based Routing. A second dimension in routing according to Figure 2.4 is advertisement-based routing (ABR). The idea is to optimize the routing of subscriptions by disseminating the information about what clients are going to publish. Similar to notification routing tables that are necessary for notification forwarding, subscription routing tables can be established based on advertisements. These advertisements also contain filters and are disseminated within the overlay with the same routing algorithms as for subscriptions. Subscription routing entries ensure that subscriptions are only forwarded in directions where brokers with publishers reside that may publish appropriate notifications. The subscription forwarding decision is true if there is an overlap between an entry in the subscription table and the filter of the incoming subscription. This way, the traffic caused by (un)subscriptions can be reduced if there are only a few publishers in the system producing notifications clients are interested in.

Figure 2.5(b) shows an exemplary scenario where broker $B_{5}$ issued an advertisements which installs subscription routing entries in the whole broker network. Afterwards, $B_{6}$ issues a subscription $s$ which matches a subset of the notifications the advertisement matches. Accordingly, $s$ is only sent towards $B_{5}$ and the respective routing table entries are created on the path. Without advertisements the subscription is disseminated within the whole network as depicted in Figure 2.5(a).

Hierarchical Routing Algorithms. The algorithms presented so far work on a peer-to-peer basis meaning that all brokers behave in the same manner 
and do not differentiate where messages come from. Hierarchical routing algorithms (HR) open a new dimension in routing (cf. Figure 2.4) by defining a dedicated root broker $R$ in the network. Every subscription is only sent towards broker $R$ which does not forward them any further. Similarly, all notifications are also forwarded at least to $R$. If a notification hits an appropriate filter on the way to $R$ it is additionally sent downwards the tree like in peer-to-peer routing.

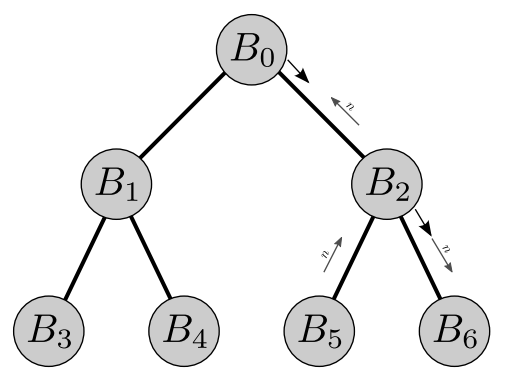

(a) Hierarchical routing

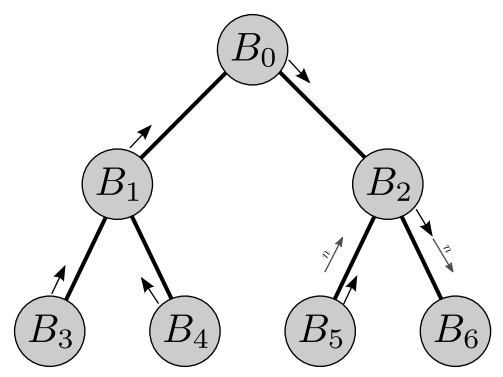

(b) Peer-to-peer routing

Figure 2.6: Hierarchical routing in publish/subscribe systems

Figure 2.6(a) illustrates hierarchical routing with $R=B_{0}$ in a scenario, where $B_{6}$ subscribed for a filter which matches a notification $n$ that is published at $B_{5}$. The black arrows represent the notification routing table entries while the gray arrows depict the delivery path of the notification. Figure 2.6(b) shows the similar situation for peer-to-peer routing without such a root broker. In this case, notifications are not sent to the root, but routing entries have to be installed in the whole broker network. Obviously, hierarchical routing reduces the number of delivered subscriptions and, therefore, also reduces the number of routing table entries. On the other hand, it increases the number of notifications due to the unnecessary delivery to $R$. This last delivery is an example for a false positive notification.

Hierarchical routing can be combined with each subscription-based routing algorithm, e.g., the application of covering-based routing may reduce the subscription flow towards the root broker if the probability for covered subscriptions is reasonable high. In Chapter 6, we recall the idea of hierarchical routing and extended it to the more general class of hybrid routing. Thus, hybrid routing algorithms substitute this routing dimension.

\subsection{Basic Assumptions}

After having provided an introduction into distributed publish/subscribe system, we finish this chapter with a list of assumptions this thesis is built on and which might not hold in general.

Local Communication. The used model assumes that the communication between clients and their local broker is for free and instantaneous. This is, for example, the case if the clients and their local broker run on the same machine. 
Cooperating Brokers. We assume that brokers behave in a cooperative manner, i.e., they act as defined by the implemented algorithms and cooperate with the other brokers in forwarding messages. Furthermore, we, thus, do not take malicious brokers into account that might try to disturb the whole system.

Moderate Dynamics. The traffic in a publish/subscribe system is mainly influenced by the behavior of clients which publish notifications and subscribe for them. A strong dynamics in this processes makes it impossible for designers to provision the system which we explain in detail in Chapter 4. Furthermore, fast changing client behavior may undermine adaptive algorithms as we introduce them in Chapter 6.

FIFO Links. For communication links in the broker overlay network FIFO behavior is required. This property is important because this way messages cannot overtake each other in a static acyclic broker overlay network. Otherwise it may happen, e.g., that an unsubscription overtakes a subscription, creating stale routing entries thereby. The FIFO property is easy to implement and required by many publish/subscribe systems that employ a single acyclic broker overlay network. A similar property is also required for brokers. Within each queue for incoming messages no overtaking, as they would appear in priority queues for example, are allowed. 


\title{
Chapter 3
}

\section{Optimizing Publish/Subscribe Systems}

\author{
Contents

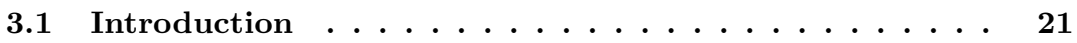 \\ 3.2 Requirements on Publish/Subscribe Systems . . . . . . 22 \\ 3.2.1 Requirements of the Clients . . . . . . . . . . . . 22 \\ 3.2.2 Requirements of the Operators . . . . . . . . . . . . 23 \\ 3.3 Optimization Objectives ............... 24 \\ 3.4 Optimizations within the Life Cycle ......... 26 \\ 3.5 Capacity Planning vs. Adaptive Algorithms . . . . . 28
}

\subsection{Introduction}

Evolution is a natural optimization process - a process of finding the best solution out of a given set of alternatives. As a child of the evolution mankind transfered this idea to technical, economical and social fields. A technical optimization consists of two major development steps, first, to formalize the optimization with the help of abstract mathematical formulas, and second, to use computers for searching for optimal solutions. Many engineers and computer scientist do follow these two steps to solve optimization challenges in their individual problem domain, such as for cluster computers [39] or staged applications [2] for example. Publish/subscribe is the problem domains we deal with in this thesis. Beside fault-tolerance and security, the optimization of publish/subscribe systems has been and still is one major motivator for researchers. It is no accident that a lot of papers have been published up to now. In this chapter, we take the bird's eye view on publish/subscribe systems to give a holistic description of what optimization of these systems means. Therefore, in Section 3.2 we start with a general requirements analysis from which we then derive possible objectives for the optimization (Section 3.3). Later in Section 3.4, we analyze the lifecyle of a publish/subscribe system including design, runtime and redesign phase. Thereby, we identify at which point the optimization algorithms can 
be implemented. Finally, in Section 3.5 we explain two strategies for optimizing publish/subscribe systems at design time resp. at runtime. Both issues are central themes of this thesis we deal with in the following chapters.

\subsection{Requirements on Publish/Subscribe Systems}

Before a software system is built, customers or/and designers define requirements the system must provide. This is a very important part of every software development process, like e.g., in the waterfall model [86]. Following, we focus on requirements on a publish/subscribe system. In principle, there are two different types of requirements, functional and non-functional, which refer to functional properties resp. non-functional properties of the system. Functional properties describe the functional core of a system, which is usually the access to information or the execution of operations. Non-functional properties focus on how the functionality of the system is provided. They include performance, security and dependability issues. In Section 2.2 , we presented interface operations of a publish/subscribe system. Their semantics can be seen as the functional requirements. The semantics is strongly related to the correctness given by Definition 2.4.2. This definition is the basic guideline for the development of many algorithms. For example, Mühl [66] formally showed the correctness of all common routing algorithms with the help of temporal logic. Such a proof is not always possible due to the complexity of the algorithm and the assumptions that have been made. Thus, in case of more complex algorithms such as self-organizing broker topologies Jaeger et al. [45] informally showed the correctness by their argumentation and by testing. In Chapter 6 , we explicitly refer on this functional requirement when we present adaptive algorithms and their compositions.

Nevertheless, having a correct system is only side of the coin. Often, every object in the daily life is not described sufficiently with its functionality. Instead, it is important how or in which way a system provides the functionality. This is what we call non-functional requirements. Concerning publish/subscribe systems, non-functional requirements are soft requirements that are not essentially needed for the core functionality, i.e., the dissemination of notifications from publishers to subscribers. Non-functional requirements include mainly QoS or performance measures and resource limitations. In the following, we further analyze these requirements by taking over the roles of both major parties of the system, the client and the operator.

\subsubsection{Requirements of the Clients}

A client of a publish/subscribe system is the entity that uses the abstract interface operations described in Section 2.2, i.e., it either acts as a publisher or subscriber. Behind this client, a human being directly or indirectly via software layer benefits from the provided functionality. Figure 3.1 depicts the situation. Considering a RSS feed application, the human being directly consumes new articles in form of notifications (left side of the figure). Other publish/subscribe systems are located in lower layers of the software stack, as for example, in event-driven architecture. Here, human beings, like managers, do indirectly profit from up-to-date information and increased responsibility of the business 


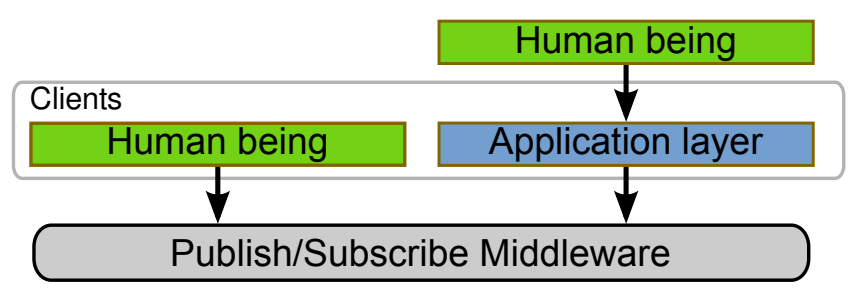

Figure 3.1: Human being and software layers

provided by publish/subscribe communication. In this case, the actual clients of the system are applications (right side of the figure).

Regardless of whether there is direct or indirect functional coupling of the human being and the publish/subscribe system he/she has requirements on the system that directly or indirectly reach the system. Thus, these requirements are either directly defined by the human being because he/she directly uses the publish/subscribe system. Or, the requirements for the publish/subscribe level can be derived from application requirements that are inherited requirements of the client of the application. Usually, requirements are defined with the help of QoS measures which often includes performance measures for its quantitative description. Other important measures such as security and dependability we skip for this thesis. When using commercial products, all these measures may be part of a service level agreement (SLA). In publish/subscribe systems there are two major measures of clients: the notification delay and the subscription delay. Both delays describe the delay between the issuing of a message and the full effect of this message. The mentioned effect depends on the message type. A subscription issued at some broker is propagated stepwise through the broker overlay network until it reaches other subscriptions that suspend its further propagation or until a leaf broker is reached. The delay of a subscription is the time between its issuing and the end of its propagation, i.e., the latest time a broker decides to not propagate the subscription further. Similar to that, the notification delay is the time it needs to deliver a notification on the path with the highest expected delay. These delays are performance measures which describe how fast clients can expect to interact with the publish/subscribe system. While for large-scale Internet applications delays in seconds range is acceptable, as e.g., the delivery of status updates in social networks, in highfrequency/low-latency trading systems or real-time enterprises [95] milliseconds are required in order to be successful.

In general each client can have different requirements on the publish/subscribe system. This imbalance may stem from varying importance of the different clients. Contrarily, in this thesis we deal with equal clients. Therefore, we are able to define one global delay measure. In Section 4.3.1, we provide formal definitions of the delays.

\subsubsection{Requirements of the Operators}

The operator of a publish/subscribe system is either a real organization, like a university or company, or some abstract virtual organization, e.g., the set of all users of a peer-to-peer network with equal requirements. We assume that such 
an operator has the publish/subscribe system under its control. Either he/she has physical or remote access to the brokers or he/she can enforce its policy in other means. Normally, operator requirements do contain the requirements of the clients because this is the foundation for the acceptance of their system. To avoid the repetition and to separate the requirements more clearly, we do not mention the performance requirements as a requirement of the operator.

In contrast to the clients, the operator has additional requirements because he/she is responsible for the publish/subscribe infrastructure. While clients may hide behind the interface and argue from their individual interests, operators cannot deny their responsibility because they have an interest on a sustainable running system that satisfies a majority of clients. However, the major goal of the operators is cost reduction. This is either a substantial interest or it is enforced by a higher management level in a company. The mentioned costs can either be real world costs counted in money units or may also be related to other measures that are connected to things that are (morally) not acceptable, like for example, the waste of resources like energy or other capacities. For economical costs, there are generally two main cost factors in publish/subscribe systems: the operational costs, such as bandwidth and energy, and investments, such as computers and other hardware for the infrastructure. These costs are directly related to measures of the publish/subscribe system, like (i) routing table sizes which are a measure for the matching overhead and memory consumption of computers, (ii) the message traffic which can be used to estimate the overall system communication costs and (iii) the utilization of brokers and links usable to detect performance bottlenecks. The latter measure could be thought as a new category (beside costs) of operator requirements but it could also be mapped to costs since blackouts of the system may end up with right of recourse claims of the clients. Further details about these measures are explained in Section 4.3.1.

\subsection{Optimization Objectives}

When analyzing the previously described requirements on publish/subscribe systems, which are based on the two different views, we easily find conflicting requirements. Obviously, this line of conflict can be draw between the two opposite roles, client and operator. The mechanism behind is that a reduction of the costs usually leads to reduced performance and vice versa. Examples of the contradiction are: a requirement for lower delay bounds for the clients usually leads to higher costs for the operator because the requirement can only be met with faster and, thus, more expensive computer hardware. In contrast, the reduction of costs by the operator by renting a cheaper Internet connection may lead to increased delays for the clients. Nevertheless, there are also cases when this reciprocal dependency is not true, e.g., an unfavorable allocation or placement of hardware can neglect increased resources.

Figure 3.2 shows both roles, their influence on the publish/subscribe middleware and their conflicting requirements. This conflict between costs and performance is the origin to deal with the optimization of publish/subscribe systems. Thereby, a system parameter configuration has to be determined which sufficiently satisfies both parties. Parameters that are target of the optimization are broker locations and interconnection as well as the routing algorithm. In principle, these parameters can either be set automatically by an algorithm or 


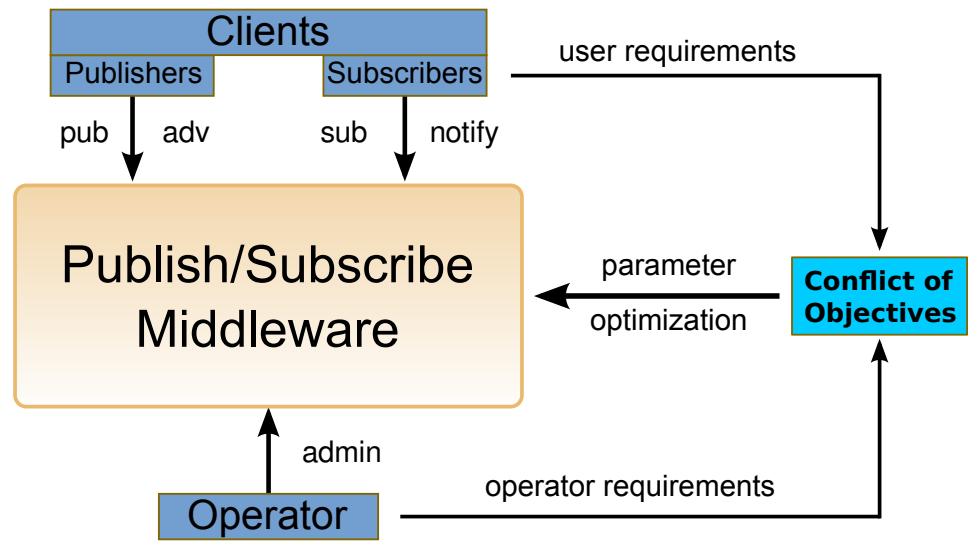

Figure 3.2: Requirements on publish/subscribe systems

by a human operator, both at runtime or at design time of the system (cf. Section 3.4). Details about these configurations will be explained in Section 4.3.2. Generally, the objectives of the optimization can be freely formalized including measures of clients and operators. Nevertheless, for such a multi-objective optimization there are some important approaches.

Maximization Principle. Known from economical theory, the maximization principle assumes that an (economic) person tries to maximize the output without exceeding a given input. Transfered to publish/subscribe systems, we look for a maximum of performance, i.e., a minimal delay, without exceeding a given amount of resources such as the number of brokers resp. computers.

Minimization Principle. Also taken from economy, the corresponding minimization principle assumes that an (economic) person tries to minimize the input without deceeding a given output. Transfered to publish/subscribe systems, we look for a minimum of resources, without exceeding a given delay. Optimization objectives following the maximization or minimization principle contain a goal function combined with constraints. Therefore, for solving advanced optimization algorithms have to be used.

Pareto-optimal Solutions. It is also possible to identify Pareto-optimal solutions, i.e., solutions that are not dominated by any other. Domination in our case is, if a solution has higher performance and requires lower resource consumption. Using this approach, it additionally needs a criterion to choose one solution from a possibly great set of Pareto-optimal solutions.

Aggregate Objective Function. Finally, a very common solution for solving multi-objective optimization problems is to reduce them to a single-objective one by using an aggregation function. This approach requires that all single objectives are weighted and accumulated which is often done by a weighted sum. Since performance and costs are contradictory, the performance measure has to 


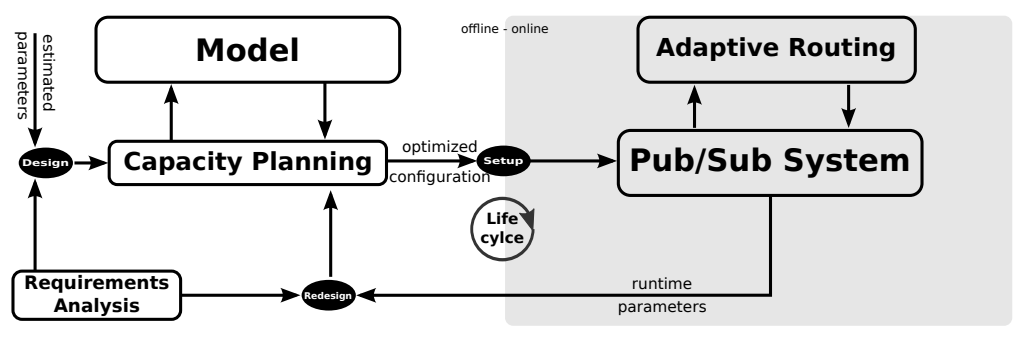

Figure 3.3: Optimizations within the life cycle of a publish/subscribe system

be inverted. After this step, the optimization can be solved by any standard or advanced optimization algorithm.

This list enumerates basic approaches to formulate objective functions for publish/subscribe systems. Designer and operators can use them for orientation when they formulate the objective function for their individual system with its individual requirements. Beside the listed approaches there is a big variety of others. In this thesis we focus on the minimization principle, especially for a case study in Chapter 5.

\subsection{Optimizations within the Life Cycle}

So far, we did not consider the fact when an optimization takes place. Therefore, we now analyze the life cycle of a publish/subscribe system which consists of three phases: the design phase, the runtime phase and the redesign phase. This life cycle definition is not equivalent to the spiral model in software development processes. We exclude the actual software development but lay the attention on the configuration resp. adaption of parameters of the publish/subscribe system.

In Figure 3.3 the life cycle of a publish/subscribe system is symbolized by the cycle in the center. Its direction shows the natural cycle starting from an offline phase when the system is designed, going to a runtime phase when the system is in production and, finally, a redesign phase when designers can correct misconceptions. The cycle is closed since after a redesign the system may again put into production. Following, we explain each phase in more detail and give starting points and approaches for optimizations.

Design Phase. The design phase is an offline phase for a publish/subscribe middleware. It is shown on the left hand side of Figure 3.3. In this phase, the task of an operator is to prepare the deployment the system meeting the requirements coming from a requirements analysis (cf. Section 3.2). Since the middleware did not run before in this specific scenario, the operator has only a fuzzy imagination of the environment the system will be deployed in. Therefore, he/she can only estimate the environmental parameters of the system, which is the client behavior and the physical network (cf. Section 4.3.2). To determine the configuration the operator may either use experience from other publish/ subscribe systems or, even better, he/she uses a capacity planning strategy for publish/subscribe systems which is introduced in Chapter 5. The capacity planning uses an underlying stochastic system model (cf. Chapter 4) to determine 
an optimized configuration. The quality of the resulting configuration heavily depends on the accuracy of the estimated environmental parameters. If they widely differ from real parameters the publish/subscribe system is in a suboptimal operating point. Nevertheless, using a model based approach is much better than if the operator estimates the entire configuration based on an implicit internal model.

Runtime Phase. After the deployment, the publish/subscribe middleware is in the runtime phase, which is depicted on the right hand side of Figure 3.3. In this phase, clients and the middleware come together. Depending on the deployment parameters, the system is more or less in an optimal state. The runtime phase is characterized by its dynamics in the behavior of clients and by changes in the underlay network. This includes fast changes like white noise as well as trends in the environmental parameters. The latter may lead to a performance degradation over time even if the initial configuration was optimal. To overcome this problem, publish/subscribe systems provide algorithms that adapt originally static configuration parameters at runtime. Such adaptive algorithms, first, enable such reconfigurations, and, second, provide a selfoptimizing behavior due to improve the performance or to reduce the required system resources. These algorithms unburden operators from either redesigning the system or to process manual reconfigurations. In Chapter 6, we introduce one of these algorithms.

Redesign Phase. Despite of the elegance of the optimization at runtime, there are some drawbacks. To avoid single point of failures, adaptive algorithms do work with decentralized decisions instead of a centralized unit. Furthermore, to reduce the overhead of the algorithm, they usually use local knowledge only. Therefore, each reconfiguration decision is based on a reduced view of the network situation and ignores effects of reconfigurations on the rest of the network. As a result, even an adaptive publish/subscribe system may become ineffective which requires a redesign when the performance drops to much. The redesign phase may run in parallel to the runtime phase when the offline calculation of a new optimal configuration requires to much time. The input of such a capacity planning are runtime parameters (cf. bottom of Figure 3.3) which are normally more accurate than the estimated parameters of the design phase. The applied capacity planning strategy is the same as in the design phase and outputs an optimized configuration. The crucial point here is how the running system which has usually another configuration can be switched to the new configuration. There are generally two ways. First, the "old" system is shut down and deployed again with the new configuration. This obviously leads to an interruption in the publish/subscribe service and requires to move all clients from one system to the other. More elegant is to have a reconfiguration scheme which allows to switch from one global configuration to another. To do this, the middleware must provide online reconfigurations of all optimization parameters. Therefore, the optimized configuration of the redesign phase must be distributed to the running system which then reconfigures autonomously. Usually, adaptive algorithms already support such reconfigurations. Hence, this global reconfiguration is possible and can be built on already implemented code. This idea is not further analyzed in this thesis. 


\begin{tabular}{|l||l|l|}
\hline Property & Capacity Planning & Adaptive Algorithm \\
\hline \hline phase & offline & online \\
\hline input parameters & complete, but outdated & local, up-to-date \\
\hline model & complete model & local model \\
\hline resources & enough resources & minimal overhead required \\
\hline results & better results & suboptimal results \\
\hline time & long & short \\
\hline
\end{tabular}

Table 3.1: Offline vs. online algorithms

\subsection{Capacity Planning vs. Adaptive Algorithms}

We have seen that at design time as well as at runtime a system optimization is possible. To emphasize the differences, we compare capacity planning and the adaptive online algorithm for self-optimization in Table 3.1. Please note that the on-/offline pair is not equivalent to algorithms used in a competitive analysis [97]. In a competitive analysis, an online algorithm that does not know the future is compared to an anticipating offline algorithm.

Capacity Planning. An offline optimization like the capacity planning is based on a complete system model to describe the behavior of the system. As inputs it needs complete information about clients and the network. Since these information have to be collected within the whole network (which takes some time) they are more or less outdated. Furthermore, a capacity planning uses complex algorithms to solve the complete model. Due to the huge search space, this process requires a lot of resources such as RAM and CPU leading to a long time for termination which is usually available since the computation can be outsourced to specialized systems such as cluster computers. Generally, the optimization result heavily depends on the dynamics of the environment. If the delay of the collection of data and the determination of the optimized configuration is smaller than the time for a significant change in the environment, such as the client behavior, the results do very likely reduce the costs or improve the performance. If the delay is too long so that the results are only valid for already outdated data, the capacity planning makes no sense. If this is the case the designer must think about accelerating the data collection or the determination of the configuration. The latter problem can be solved by stopping the optimization process earlier to get more up-to-date result. In this thesis, we assume that capacity planning lasts hours, while the system changes over day or weeks. In this case, a redesign is always reasonable. In Chapter 5 , we introduce a capacity planning strategy in detail.

Adaptive Algorithms. In opposite to the capacity planning, adaptive algorithms are online algorithms. The algorithms we consider are focused on the optimization of the system. Thus, we also call them self-optimizing algorithms. In a distributed system these algorithm mostly have a limited model of their environment and use local environmental information as inputs. Local in this case means that nodes only use information of $n$-hop neighbors. Due to the spatial distribution of the nodes, these information must be exchanged 
by messages which generate additional load. The information exchange can be realized by periodic pushes or pulls of the data as well as by event-driven dissemination of information if "something new happened". The minimal case for this local information collection is when nodes do only use the information they can directly measure $(n=0)$. The other extreme $(n=\infty)$ is when data from the whole network is collected which is equivalent to the capacity planning case. For each algorithm, the selection of $n$ is crucial for the optimization result. On the one hand, to much traffic for the collection of information and the calculation of the result should be avoided. On the other hand, the local behavior based on a reduced model should not disturb the self-optimization. Compared to offline optimizations, in online optimizations the resources are much more limited. In order to avoid overloading of single nodes, the algorithms are kept as simple as possible. The optimization process from the global view is generated in a self-organizing manner meaning that nodes do interact locally, similar to the information collection. This self-organizing behavior to reach a macroscopic behavior with local interaction is sometimes called "stigmergy" [36]. Formal methods, but more often evaluations by tests, must show if the expected macroscopic behavior really occurs. To evaluate the performance of the algorithm, the optimization results reached by the adaptive algorithm can be compared with a real-time capacity planning without any delay for the collection and determination of the optimal configuration. This is a similar approach as the competitiveness $\sigma$ for on/offline algorithms or the relative performance guarantee $\rho$ for approximation algorithms.

In this thesis, we mainly focus on one adaptive algorithm for publish/ subscribe systems which adapts the routing algorithm based on 0-hop neighbor information. Only for the coordination direct neighbors are involved. Details about this algorithm are explained in Chapter 6. 


\section{Chapter 4}

\section{Stochastic System Model}

\section{Contents}

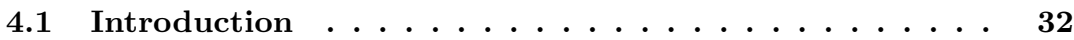

4.2 Related Work . . . . . . . . . . . . . . 33

4.3 Inputs and Outputs of the System Model . . . . . . 35

4.3 .1 System Outputs . . . . . . . . . . . . . . . . . . . . . . . . . .

4.3 .2 System Inputs . . . . . . . . . . . . . . . . . . . . . . . . . . . . . . . . . .

4.4 Derivation of the System Model . . . . . . . . 42

4.4 .1 Client Behavior Model . . . . . . . . . . . . . . 42

4.4.2 Routing Algorithms . . . . . . . . . . . . . . . . . . . . . . . . . . . . . . 45

4.4 .3 Broker Model . . . . . . . . . . . . . . . . . 47

4.4.4 Model for Physical Links and Routers . . . . . . . . . . . . 48

4.4.5 Auxiliary Measures . . . . . . . . . . . . . . . . . . 48

4.4 .6 Routing Table Size . . . . . . . . . . . . . . . 50

4.4.7 Forwarding Probabilities of Messages . . . . . . . . . . . . . 54

4.4 .8 Traffic . . . . . . . . . . . . . . . . . 68

4.4 .9 Utilization and Delay . . . . . . . . . . . . . 71

4.5 Evaluation ................... 73

4.5 .1 Simulation . . . . . . . . . . . . . . . . . . . . . . . . . . . . . . . . . . .

4.5 .2 Insights . . . . . . . . . . . . . . . . . . 74

4.5 .3 Hierarchical Routing . . . . . . . . . . . . . . . . . 79

4.6 Generalizations and Extensions . . . . . . . . 88

4.6.1 Multidimensional Notifications/Subscriptions . . . . . . . . 88

4.6.2 Variable Number of Attributes . . . . . . . . . . . . . . 93

4.6.3 Utilization and Delay . . . . . . . . . . . . . . . . . . . 95

4.7 Discussion ......................... 96 


\subsection{Introduction}

With the increasing popularity of publish/subscribe systems and their gradual adoption in mission critical areas [41], performance and scalability issues are becoming a major concern. System designers and deployers are often faced with questions such as: What performance would the system exhibit for a given deployment topology, configuration and workload scenario? What would be the expected notification and subscription delays as well as the utilization of the various system components (brokers, network links, etc)? What maximum load (number of publishers and subscribers, notification publication rates) would the system be able to handle? What would be the optimal number of brokers and the optimal system topology? Which components would be most utilized as the load increases and are they bottlenecks? To answer such questions, techniques for predicting the system performance as a function of its configuration and workload are needed. Therefore, in this chapter we present a system model of distributed content-based publish/subscribe systems that covers all major aspects of such systems allowing to answer all the latter questions. The presented model is initially based on Jaeger et al. [44] who laid the very foundation for a stochastic system model of content-based publish/subscribe systems. In [68] and [93], we stepwise extended the model by releasing many of the former restrictions and by adding new performance aspects. The model presented in this thesis goes beyond and provides a general model covering all major aspects of a distributed publish/subscribe system. Beside the performance prediction, this model is foundation for designing and deploying optimized infrastructures presented in Chapter 5.

This chapter starts with the related work presented in Section 4.2 where the state of the art in modeling publish/subscribe systems is discussed. In Section 4.3 we present the general structure of the system model which is the abstract view of a distributed publish/subscribe system. Similar to system models in many other scientific disciplines we first focus on the identification of inputs and outputs of the model. The inputs of the publish/subscribe model include the environmental parameters such as the behavior of clients as well as configuration parameters of the publish/subscribe system such as the network topology or the applied routing algorithm. On the other hand, the output of the model contains many Quality-of-Service (QoS) measures such as various traffic, utilization and delay measures. Based on this information we derive the stochastic system model in Section 4.4 leading to a set of formulas bringing inputs and outputs together. This is one major contribution of this thesis. The system model describes the average system behavior of a distributed content-based publish/ subscribe system in steady-state. During the stepwise derivation of the formulas, we plot interesting measures to point out insights which are sometimes intuitive general knowledge about publish/subscribe systems but sometimes also surprising. In Section 4.5, we show how powerful the model is. Therefore, we provide interesting insights with a special focus on routing algorithms. Furthermore, we evaluate the model giving a detailed analysis of hierarchical routing. In Section 4.6, we explain how the applied restrictions of the model can be skipped leading to generalized formulas. These extensions include a more general data and filter model and more sophisticated models for the clients behavior and the message processing and transmission in brokers and physical components. The chapter is completed by a discussion in Section 4.7 . 


\subsection{Related Work}

There is a variety of publications that deal with the modeling of publish/ subscribe systems. Following, we explain all important papers and discuss their individual drawbacks and weaknesses.

Castelli et al. [23] present a simple analytical model of publish/subscribe systems. The authors provide closed form analytical expressions for the overall network traffic required to disseminate subscriptions and propagate notifications, as well as for the message forwarding load on individual system nodes. However, the model, at least in its presented form, assumes that subscribers and publishers are uniformly distributed over a balanced tree in which each inner node has the same number of children. The only investigated algorithm is identity-based routing in its non-hierarchical form while the filter model is similar to channels. The system behavior is not modeled by stochastic processes but by probabilities that a broker is a publisher or subscriber.

Bricconi et al. [14] present a model of the JEDI publish/subscribe system that is mainly used to calculate the number of notifications received by each broker using a uniform distribution of subscriptions. To model multicast communication, the authors introduce a spreading coefficient that models the probability that a broker at a given hop-distance from the publishing broker receives a published notification.

Baldoni et al. [5, 6] propose an analytical model of distributed computation based on a publish/subscribe system. The system is abstracted through two delays, namely the subscription/unsubscription delay and the diffusion delay which are assumed to be known. However, the proposed model is only used to calculate the number of notifications missed by subscribers due to high network delays. Performance metrics such as notification delays and broker utilization are not considered.

A basic high-level cost model of publish/subscribe systems in mobile Grid environments is presented by $\mathrm{Oh}$ et al. [71]. It compares push-based (publish/ subscribe) pull-based (request/reply), and polling dissemination. This model, however, does not provide much insight into the behavior of the system since it is based on the assumption that the publish/subscribe cost and time delay per notification are known.

He et al. [38] use probabilistic model checking techniques and stochastic models to analyze publish/subscribe systems. The communication infrastructure (i.e., the transmission channels and the publish/subscribe middleware) are modeled by means of probabilistic timed automata which is then used as an input for PRISM ${ }^{1}$, a probabilistic model checker. The analysis considers the probability of message loss, the average time taken to complete a task and the optimal message buffer sizes. The main drawback of the presented approach is the explosion of the state space and that it assumes a publish/subscribe system with a central broker.

Kounev et al. [54] present a methodology for workload characterization and performance modeling of distributed event-based systems. A workload model of a generic system is developed and analysis techniques are used to characterize the system traffic and to estimate the mean notification delivery latency. For more accurate performance prediction queueing Petri net models are used.

\footnotetext{
${ }^{1}$ http://www.prismmodelchecker.org/ (last visit: 2012-06-04)
} 
While this technique is applicable to a wide range of systems, it relies on monitoring data obtained from the system and it is therefore only applicable if the system is available for testing. Furthermore, for systems of realistic size and complexity, the queueing Petri net models would not be analytically tractable and, thus, one would have to resort to simulation.

The work of Pongthawornkamol et al. [81, 82] mainly focuses on subscriber admission control. They also use a content-based publish/subscribe system with a data and filter model similar to ours and queueing theory to determine link and broker delays. Nevertheless, in the calculation of the content distribution their approach seems not very strict when they simply add probability distributions. Also, the authors leave out important aspects, such as dynamic subscriber behavior, the support of different routing schemes, such as covering-based routing, and the underlay network.

In his Ph.D. thesis [40] Henjes developed and evaluated a performance model for various JMS implementations based on extended queueing theory. He is mainly focused on the processing time of messages in a central server setting. Thereby, he considers parameters such as the number of filters and the replication factor. Because Henjes focuses on real JMS implementations his approach is limited in terms of distribution, where he only supports publisher-side and subscriber-side JMS server replication. Thus, routing aspects as well as filter propagation as they occur in broker-based publish/subscribe system are not considered.

The paper of van Vliet et al. [102] is based on Jaeger et al. [44] which was extended by dynamic aspects such as a fluctuating birth and death rates for subscriptions. This way, daily cycles or sudden steps can be investigated. The presented model determines the time behavior of the control traffic which allows a deeper view in the system behavior than a steady-state analysis. Additionally, van Vliet et al. also consider finite populations of clients as well as roaming clients and advertisements. Despite these advantages, the model does not consider fluctuating publication rates and the model is limited to hierarchical identity-based routing. Furthermore, performance measures like delays are not supported. Nevertheless, with the presented approach the authors introduce a new dimension in the analysis of publish/subscribe systems. Since this dimension is orthogonal to our research direction, a combination is possible with some effort.

Beside the discussed papers there are many others which use simplified models for the evaluation of their approaches (e.g. [10] and [24]). To summarize, most existing approaches to performance analysis of publish/subscribe are focused on specific system configurations that are evaluated by means of time- and resource-intensive simulations. Such evaluations are expensive especially when large-scale systems with multiple alternative configurations and workloads have to be considered. While a few analytical models of publish/subscribe systems have been proposed, they impose a number of restrictive assumptions which limit their practical applicability. Furthermore, most approaches typically do not consider important performance-relevant system metrics such as the end-toend notification and subscription delays, but instead are focused on lower level metrics such as the routing table sizes of brokers and their message throughput. 
environment

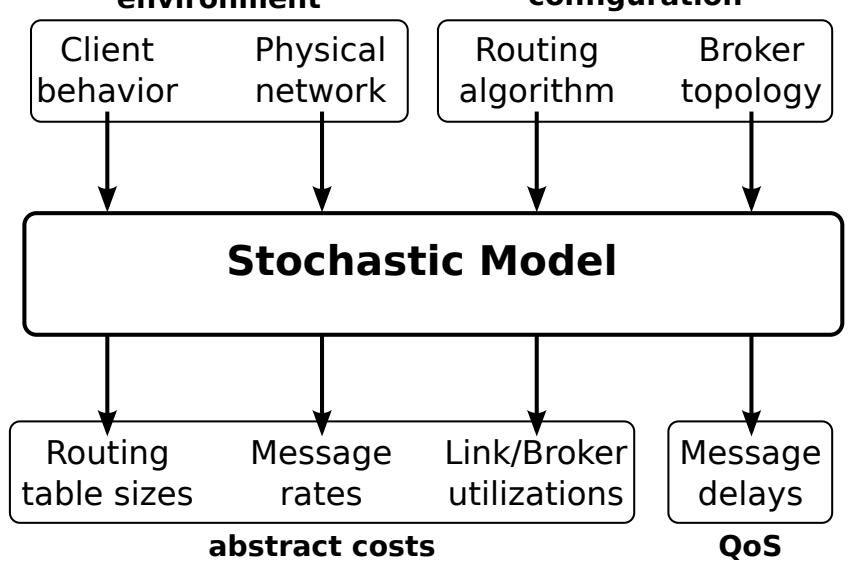

Figure 4.1: General system model

\subsection{Inputs and Outputs of the System Model}

A system model is an abstraction of a real system. Depending on the intention of the creator, there are a lot of possible models of one system. The intention to create a publish/subscribe system model is to predict the behavior of a real publish/subscribe system in terms of performance measures and costs. With such a model, designers can investigate the behavior of systems at various configurations without the need for experimenting with the real system. Furthermore, it is the foundation for our capacity planning strategy presented in Chapter 5. In this section, we will identify and describe inputs and outputs of the system model, while we postpone some of its internals to Section 4.4. We will start with the system outputs. Here, we introduce important measures of performance and costs. After that, the inputs are derived by analyzing existing publish/subscribe systems. In advance, an overview about the model and its inputs and output is given in Figure 4.1. Details are explained in the following subsections.

\subsubsection{System Outputs}

The outputs of the system model are centered around abstract costs and performance resp. QoS measures which we use as synonyms. As described in Chapter 3, costs are more in the interest of the operator, while performance is important for clients of the system. We use the term abstract costs which are more technically compared to real-world costs which are usually counted in money units. It is up to the operator to map the abstract costs to real ones. As system outputs we provide four important measures of a publish/subscribe system, namely routing table sizes of brokers, message traffics and utilizations of brokers and links as well as delays.

The expected (notification) routing table size $X^{s}(B)$ is a unitless measure individually provided for all brokers. Since each routing table entry requires a certain amount of memory to store filter and destination, this measure is pro- 
portional to the memory requirements of the machines. Furthermore, the size of the routing table correlates with the matching costs and the costs to determine filter similarities in covering-based routing, for example. This matching costs are further correlated to the CPU utilization. In case that advertisementbased routing is used, also a subscription routing table exists with the related size $X^{a}(B)$.

The message traffics express the amount of publish/subscribe messages, such as notifications and control messages, per time unit. A traffic can either refer to brokers $\nu_{i n}(B)$ or physical links $\nu_{i n}(\bar{l})$. In case of the message traffic of a broker, we count the incoming traffic the broker has to handle. This is relevant for the operator to dimension the computing power of the machines. Since most physical links are bidirectional, the message traffic on links subsumes the traffic in both directions. With the help of the message size this measure can be mapped to a required bandwidth. In WANs and other infrastructures, the bandwidth is directly connected to real communication costs. Another closely related measure is the utilization. It is a unitless measure which can refer to brokers $U(B)$ as well as links $U(\bar{l})$ and is determined by the ratio between the message traffic and a given capacity. The utilization of brokers and links allows to determine bottlenecks in the infrastructure.

For clients we defined two important measures that are the delay for notifications $\Delta^{n}$ and subscriptions $\Delta^{s}$. These measures are commonly used as QoS metrics in publish/subscribe systems. Since their definitions must not be ambiguous, we discuss small but notable differences between various definitions of delays in publish/subscribe systems in the following.

Delay. A delay can be defined by the time difference of the occurrence of two causally linked events, a cause and an effect. Assuming that a system has no anticipative behavior, the delay can be determined by the difference of the timestamps of both events: $d=t_{e}-t_{c}$. Examples for pairs of such events are a request and a reply event in a client/server system or a simple send and a receive event when passing a message. In the first case, delays can be locally measured with a physical clock whose drift must be limited by the intended measuring accuracy. For distributed delay measuring, like in the send/receive example, synchronized clocks are necessary which must be synchronized with protocols such as NTP. In the analysis we abstract from this problem and refer to the global time. In fact, this is only available for simulation environments like we use in this thesis.

To measure the delay in publish/subscribe systems, we need to define cause and effect events as mentioned above. The cause event can be exactly defined. It is the issuing of a message, i.e., the issuing of a subscription or the publication of a notification. The definition of the effect event is more challenging. For both message types one issuing of a message may cause several consequential propagations. These propagations include simple forwardings as well as splits of the message path into any number of new paths. This is what the blue delivery tree depicts in Figure 2.2. Following, we will show that beside the easiest approach which considers the last delivery of all consequential messages, there are several possibilities for defining the effect event more exactly. 


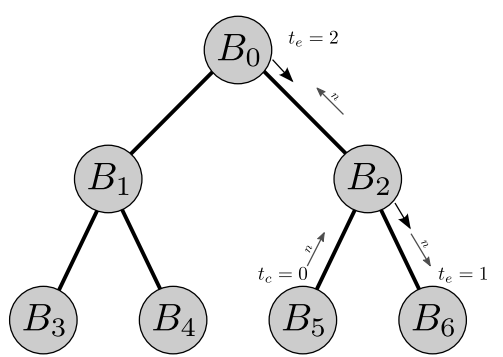

(a) Notification delay

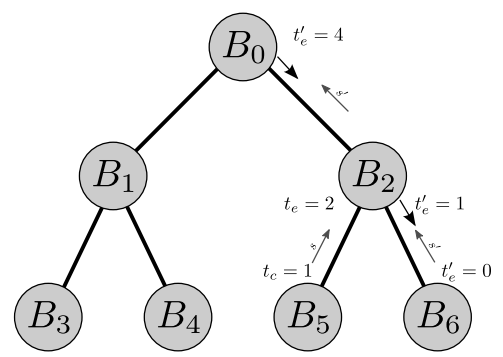

(b) Subscription delay

Figure 4.2: Definitions of notification and subscription delay

Notification Delay. When a notification has been published, the routing algorithm ensures that this notification reaches all interested subscribers. For the notification delay we discuss two possible definitions of the effect event which differ in the time when we count a notification as being delivered. A notification is delivered when ...

1. there is no further delivery of this notification within the network $\left(d_{F L}^{n}\right)$.

2. it reaches the last interested subscriber $\left(d_{F I L}^{n}\right)$.

In the first case, we consider all deliveries of the notification on the delivery tree built by the routing algorithm. The latest time when this notification reaches a leaf of the delivery tree is $t_{e}$ with a resulting $d_{F L}^{n}$. In case that no delivery takes place at all it holds that $t_{c}=t_{e}$. This latest delivery time is very easily to determine if the distributed publish/subscribe system runs in a simulation environment with a global view on the messages in transit. When the last notification message is delivered $t_{e}$ is counted. In a real distributed publish/ subscribe system with nearly synchronized clocks we may store a timestamp as well as an identifier for each incoming notification that is not further forwarded. After collecting all these data items one can sort them and take the largest timestamp to determine $t_{e}$ and $d_{F L}^{n}$.

The second approach takes into account that a publication is only interesting for subscribers with matching filters. The latest delivery time to such a subscriber is $t_{e}$ with a resulting $d_{F I L}^{n}$. Especially in case of a publish/subscribe network using the flooding algorithm, there are lots of false positive (FP) deliveries of notification messages which increase the calculated delay. This extreme case shows that due to the handling of false positives the delay of approach (1) is equal or greater than the one of approach (2): $d_{F L}^{n} \geq d_{F I L}^{n}$.

Figure 4.2(a) exemplary depicts the difference between approach (1) and (2). The overlay consisting of seven brokers uses hierarchical routing. A message that has been published at broker $B_{5}$ at time $t_{c}=0$ is delivered via $B_{2}$ to broker $B_{6}$ and to the root broker $B_{0}$. Since the latter unnecessary delivery has a greater timestamp $\left(t_{e}=2\right)$ than the delivery to an interested subscriber at broker $B_{6}\left(t_{e}=1\right)$, the first approach results in $d_{F L}^{n}=2-0=2$ which is greater than approach (2) with $d_{F I L}^{n}=1-0=1$.

For the analysis we focus on the definition (1), i.e., we define the delay of a notification as the time from its publication until there is no further delivery of 
this notification within the network. This definition actually refers to a single message delivery. To have a global measure we define the notification delay of a publish/subscribe system $\Delta^{n}$ as the maximum broker delay of all brokers in the system where the broker delay is the average delay of all notifications published at this broker.

Subscription Delay. For the subscription delay we again discuss several possible definitions of the effect event which differ in the time when we count a subscription as being active. Generally, there are two types of definitions. One is that we count the delay of a subscription if it is no longer delivered. This is the case if the subscription reaches a leaf broker (or the root broker in hierarchical routing) or if its delivery is inhibited due to identical or covering filters in the routing table.

The second type of definitions considers the activity of these inhibiting subscriptions. Only if they are active themselves the delay is counted. As an illustration of the difference Figure 4.2(b) shows a network with hierarchical routing. While the last delivery of subscription $s$ is at $t_{e}=2$, the subscription delay of $s$ is counted when the inhibiting subscription $s^{\prime}$ is reaching the root broker $B_{0}$ at $t_{e}^{\prime}=4$. Generally, we get the following definitions. An issued subscription is active when ...

1. it reaches the last leaf broker $\left(d_{S R}^{s}\right)$

2. it reaches a broker with an identical active subscription $\left(\tilde{d}_{I B R}^{s}\right)$

3. it reaches a broker with a covering active subscription $\left(\tilde{d}_{C B R}^{s}\right)$

4. it reaches a broker with a covering merge of active subscriptions $\left(\tilde{d}_{M B R}^{s}\right)$

5. it reaches a broker with an identical active subscription and the identical subscription is already active at the last leaf broker $\left(d_{I B R}^{s}\right)$

6. it reaches a broker with a covering active subscription and the covering subscription is already active at the last leaf broker $\left(d_{C B R}^{s}\right)$

7. it reaches a broker with a covering merge of active subscriptions and the covering subscriptions are already active at the last leaf broker $\left(d_{M B R}^{s}\right)$

The first four delays could be summarized under "there is no further delivery" as in definition (1) of the notification delay. Nevertheless, we distinguish between, $d_{S R}^{s}, \tilde{d}_{I B R}^{s}, \tilde{d}_{C B R}^{s}$, resp. $\tilde{d}_{M B R}^{s}$ because the delivery event is in fact different. Each of the mentioned delays can be measured directly if the related routing algorithm (SR, IBR, CBR, MBR) is active. Since this is the most practical way to measure the delay of subscriptions, we use it in this thesis. The problem here is that if we use it to compare different routing algorithms, we base the comparison on different measures. A correct measurement of the delays would require additional computational effort (checking the covering relation while SR) or additional traffic (forwarding of covering subscriptions in CBR) which falsifies the measurement since this effort would not occur if we would not measure. Generally, the mentioned delays can be ordered as follows: $d_{S R}^{s} \geq \tilde{d}_{I B R}^{s} \geq \tilde{d}_{C B R}^{s} \geq \tilde{d}_{M B R}^{s}$, i.e., in simple routing we measure a 
higher delay than in identity-based routing even if we use the same measurement method and the same parameters.

The second part of delays in the enumeration are more theoretical ones. Their definitions also mention the activity of a subscription which usually depends on other subscriptions, e.g., a covered subscription is only active if the covering subscription is already active. This is a recursive definition where the beginning of recursion is when a subscription reaches all leaf brokers (cf. Definition 1). These dependencies can be defined in different ways, namely by identity, by covering and by merging, leading to the different definitions. Delays defined in this way cannot be measured natively but require a complex analysis of all messages that are in progress. The delay $d_{M B R}^{s}$ is in fact the "real" subscription delay because it considers all reasonable similarities of subscriptions. The mentioned delays can be ordered as follows: $d_{I B R}^{s} \geq d_{C B R}^{s} \geq d_{M B R}^{s}$.

To better see in which situations one or the other delay is smaller or greater than the real delay $d_{M B R}^{s}$ we consider now different disjunctive cases when a subscription is arriving at a broker:

1. There is no covering relation between the arriving subscription and all other subscription of the routing table.

2. There is at least one subscription in the routing table that is identical to the arriving one. In this case, we also differentiate between the case when the identical subscription itself is already active or not.

3. There is at least one subscription in the routing table that covers the arriving one. In this case, we also differentiate between the case when the covering subscription itself is already active or not.

4. There is a subset of subscriptions in the routing table that together cover the arriving one. In this case, we also differentiate between the case when the covering subscriptions themselves are already active or not.

For each situation we investigate if we measure the correct value or if the measured value is greater $(\uparrow)$ or lower $(\downarrow)$ then the correct value. This analysis we filled into Table 4.1. It shows that compared to the real delay $d_{M B R}^{s}$ only $\tilde{d}_{M B R}^{s}$ is strictly smaller, because it counts a subscription to be active even if the merger is still in transit. On the other hand, $d_{S R}^{s}$ is always strictly greater than the real value since it counts the delay when the subscription reaches the leaf brokers even if it was active before through a covered subscription for example. The delays $d_{C B R}^{s}$ and $d_{I B R}^{s}$ are indifferent according to $d_{M B R}^{s}$ meaning that they may be greater or smaller depending on the probability of the different situations. By bringing all comparisons together, one can derive the partially ordered set of delays depicted in Figure 4.3.

As already mentioned earlier, our way to calculate the delay is to use the timestamp when no further delivery of this subscription within the network is taking place. As for the notification delay, we define a global measure $\Delta^{s}$ which is the subscription delay of a publish/subscribe system. It equals the maximum delay of all brokers in the system, where the broker delay is the average delay of all subscriptions issued at this broker. 


\begin{tabular}{|l||c||c|c||c|c||c|c|}
\hline \multicolumn{1}{|l|}{ Delay } & \multicolumn{1}{l||}{ nothing } & \multicolumn{2}{c||}{ ident. sub. } & \multicolumn{2}{c||}{ covering sub. } & \multicolumn{2}{c|}{ covering merger } \\
type & & active & not a. & active & not a. & active & not a. \\
\hline \hline$\tilde{d}_{M B R}^{s}$ & $=$ & $=$ & $\downarrow$ & $=$ & $\downarrow$ & $=$ & $\downarrow$ \\
\hline$\tilde{d}_{C B R}^{s}$ & $=$ & $=$ & $\downarrow$ & $=$ & $\downarrow$ & $\uparrow$ & $\uparrow$ \\
\hline$\tilde{d}_{I B R}^{s}$ & $=$ & $=$ & $\downarrow$ & $\uparrow$ & $\uparrow$ & $\uparrow$ & $\uparrow$ \\
\hline$d_{M B R}^{s}$ & $=$ & $=$ & $=$ & $=$ & $=$ & $=$ & $=$ \\
\hline$d_{C B R}^{s}$ & $=$ & $=$ & $=$ & $=$ & $=$ & $\uparrow$ & $\uparrow$ \\
\hline$d_{I B R}^{s}$ & $=$ & $=$ & $=$ & $\uparrow$ & $\uparrow$ & $\uparrow$ & $\uparrow$ \\
\hline$d_{S R}^{s}$ & $=$ & $\uparrow$ & $\uparrow$ & $\uparrow$ & $\uparrow$ & $\uparrow$ & $\uparrow$ \\
\hline
\end{tabular}

Table 4.1: Comparison of delays

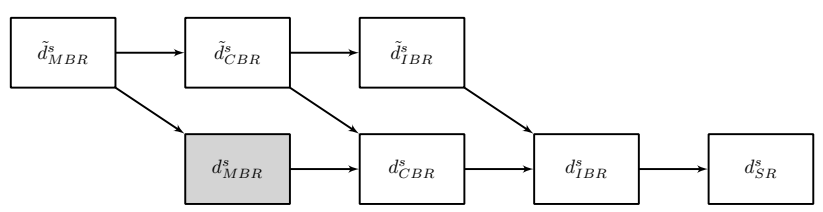

Figure 4.3: Partially ordered set of delays

\subsubsection{System Inputs}

To derive the mentioned outputs of the system, it is necessary to identify inputs of the system model. Often in literature, like e.g., in [69], the inputs of a system are divided into two types: regular inputs and control inputs. While regular inputs refer to environmental changes, such as request patterns, disturbances, etc., control inputs can be directly influenced by a human or an (automatic) controller. In this thesis, we use the terms environment and configuration to refer to these two types of inputs.

As the environment of a system we mean all inputs that cannot be influenced by the system. For example, the request patterns of a web server or the crash of a peer in a peer-to-peer network is nothing the system itself can influence directly. These inputs do not provide an open interface so that the system can manipulate them. This does not exclude that an indirect influence is possible. Recalling the web server example, the request patterns may indirectly changed by providing websites with a better or worse usability. Applying such strategies requires an internal model of the environment.

On the other hand, the configuration are inputs that can be influenced by the system itself or by a human controller. Thereby, we can differentiate between configuration parameters that can be set at system startup and parameters that can be influenced at runtime. In software systems we often have the possibility to create new configuration parameters that have been fix before. Furthermore, we are able to convert a design time configuration parameter into a runtime configuration parameter which enables the system to be adaptable.

Environment. For publish/subscribe systems we identified three important environmental parameters: (i) the client behavior which describes how clients 
use the interface operations of the notification service, (ii) the physical network which represents physical routers and links, and, (iii) faults of hardware units, e.g., brokers or network links. The last environmental parameter is mentioned here to be complete, but faults are not considered in the presented stochastic system model.

The first input parameter, the client behavior, comprises the distribution of clients, their individual mobility and, their publication rate and interests. These parameters are externally set and cannot be influenced by the operator directly. Nevertheless, clients are the originators of all processing and communication within the publish/subscribe network and they are the major motivation for defining performance measures. The analytical model does not deal with clients directly but for each broker the arrival and lifetime of subscriptions as well as the arrival of notifications is modeled by stochastic processes, whose parameters either have to be measured or estimated.

In contrast to the overlay topology, the physical network is usually a static input parameter which can either be known, e.g., in a company, or it has to be determined by network scans. For simulating an Internet-scale publish/ subscribe system it is possible to use topology generators as we do it in this thesis. In fact, the physical network comprises all layers resp. protocols below the overlay network such as TCP/IP. We abstract from this by defining that the physical network is described by a set of routers and interconnecting links where both are limited by a certain capacity. The processing of messages is modeled by queueing systems and, thus, influence the output of the analytical model.

Configuration. The configuration of a publish/subscribe system mainly consists of two parameters: (i) the routing algorithm which is used by brokers to guarantee a correct notification distribution, and, (ii) the overlay topology which is built on top of the physical layer and consists of brokers and logical links.

The routing algorithm implements the functionality of a notification service by disseminating messages between clients. Depending on the applied algorithm, different strategies for message delivery are applied which leads to different message traffics and routing table sizes. For the analytical model, flooding, simple routing, identity-based routing, covering-based routing, and merging-based routing optionally combined with advertisements are considered. The main challenge in modeling is to put the dynamic behavior into stochastic characteristics like expectation values of forwarding of messages or covering of subscriptions. The routing algorithm is an input parameter that can be set by the designer of a system and, therefore, it can be used for optimization purposes.

The routing algorithm is applied to a logical network, the overlay topology. Usually, this overlay can be set independently if there is no restriction in the positioning of brokers. Therefore, it plays a central role in optimization. Formally, the overlay network is an acyclic graph that is embedded into the physical network meaning that each broker is associated with a physical node and each overlay link can be mapped to one or more physical links including its corresponding nodes. The overlay network influences the analytical model in two ways. First, it determines the available routes of messages and, therefore, has to be considered in modeling the dynamic behavior of the routing algorithm. Second, the processing and transmission times are directly assigned to brokers 
and links. While the processing of messages on a broker is directly modeled as a queueing system with an average service rate, the transmission of messages on a link is determined by its containing physical links and routers.

\subsection{Derivation of the System Model}

The described system inputs fully determine the system outputs. In this subsection, we stepwise derive a set of recursive formulas which build up the stochastic system model of a publish/subscribe system. We analyze a system that is not overloaded, i.e., that no broker or link has to deal permanently with a higher traffic than its capacity allows. Furthermore, we focus on publish/subscribe systems in steady state meaning that birth and deaths of subscriptions compensate each other leading to a nearly constant number of active subscriptions. In this steady state also the number of publications per second is approximately constant.

Figure 4.4 provides a qualitative overview about the derivation steps. It contains all major dependencies starting from the system inputs (blue), via auxiliary values (gray), leading to the system outputs (brown). For an improved readability we left out some minor dependencies. For example, the overlay configuration depends on the underlay since brokers can only be positioned on physical nodes. Also, the routing configuration makes only sense for configured overlay links. Figure 4.4 additionally gives an overview in which sections the related derivation steps can be found.

The first step of the derivation is the introduction of an appropriate and general model of the client behavior including the underlying data model for notifications and filter model for subscriptions. This is presented in Section 4.4.1. Furthermore, other inputs such as the routing configuration and the model of brokers and of the physical network are provided in Sections 4.4.2, 4.4.3 and 4.4.4. The overlay configuration is not explicitly described here since we simply have an acyclic overlay topology as described in the introduction of the thesis. The main part of the derivation begins with the introduction of important auxiliary measures in Section 4.4.5. They build the foundation for all further calculations. Based on these values, we are able to derive the first performance measure, the routing table sizes for brokers in Section 4.4.6. As the overview figure shows, the routing table strongly depends on the routing configuration and the overlay configuration. In Section 4.4.7, we present a very important result for the understanding of publish/subscribe systems, the forwarding probabilities. We derive them for all message types, i.e., notifications, subscriptions and advertisements, and for all major routing algorithms. Based on these results we complete the derivation of the system model by focusing on the message traffics, utilizations and the delays in Sections 4.4.8 and 4.4.9. The delay calculations depend on the physical network that we introduced beforehand.

\subsubsection{Client Behavior Model}

The client behavior model describes what actions the clients of a publish/ subscribe system perform over time. These action contain the usage of the publish/subscribe interface as described in Section 2: publish, (un)subscribe, 


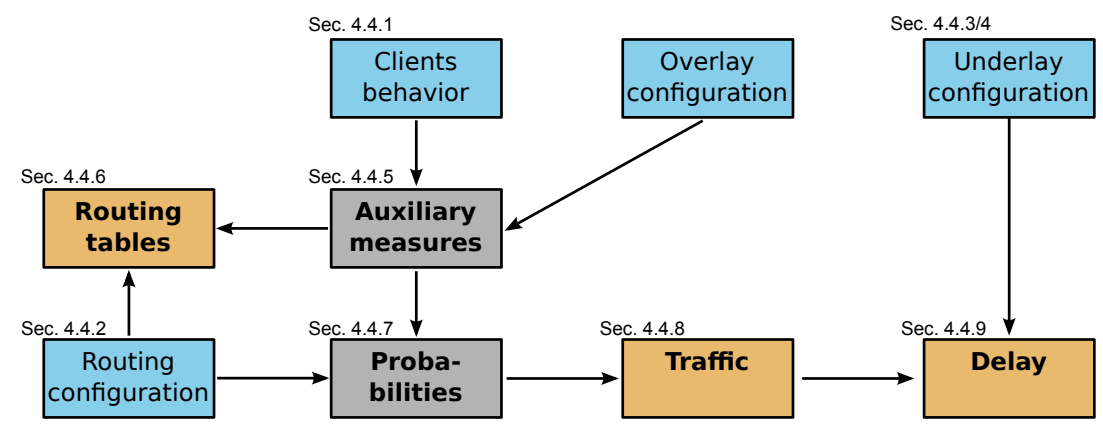

Figure 4.4: General modeling approach

(un)advertise. Following, we describe the data and filter model (which is a reduced form of that introduced in Section 2.4.1) and the dynamic aspects of the clients' actions. For a better understanding, we also give a short introduction in Markovian chains.

Data and Filter Model. For the sake of compactness of the presentation, we restrict the introduced model of Section 2.4.1 to one dimension. This restriction will be skipped later in Section 4.6. Thus, each notification $n$ has only a single numeric attribute $x$ from the set of real numbers $\mathbb{R}$. On the other hand, a filter $F$ consists of one interval $[a, b]$ with two numeric bounds $a$ and $b$ which are both real numbers. In case that $b>a$ we invert the limits, thus, always getting a natural interval. As already mentioned before, these filters can either be part of a subscription or of an advertisement.

For the special case of identity-based routing in this analysis we ignore the actual content of notifications and filters. Instead, the matching and routing only deals with the filter class, which finally equivalents to the attribute (filter) name. In the one-dimensional case, as we use it in this section, a subscription always matches a notification. Please note that this is what the publish/subscribe middleware is internally doing. The user interface as we described in Section 2.2 remains the same. Therefore, the content-based filtering must be done locally.

Dynamics. Instead of dealing with clients directly, we deal with independent publications at the brokers. For the publications we denote $\omega(B)$ as the publication rate of broker $B$. The interarrival times of two publications are independent and are modeled using an exponential distribution. Each time a notification is published, the content is selected according to the data model. The attribute value $x$ is randomly chosen described by a probability density function $f^{n}(x)$ which is identical for all clients.

The subscription interarrival times are modeled using exponential distributions. For subscription lifetimes, we also have an exponential distribution with another parameter. We denote with $\lambda^{s}(B)^{-1}$ the mean interarrival time and with $\mu^{s}(B)^{-1}$ the mean lifetime of subscriptions at broker $B$. Together with the assumption that there is no principle limitation of the number of active subscriptions, this model leads to a $M / M / \infty$ queueing system (according to Kendall's notation [48]) which we explain in the next paragraph. We call $\lambda^{s}(B)^{-1} s u b$ - 


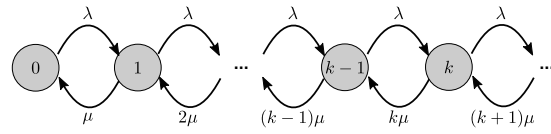

(a) $M / M / \infty$ queueing system

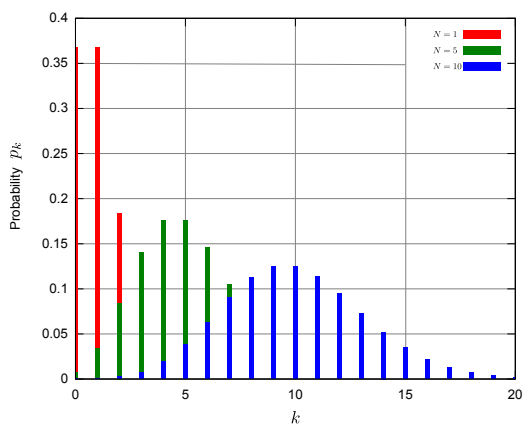

(b) State distribution of a Poisson process

Figure 4.5: Markovian chains

scription (birth) rate and $\mu^{s}(B)$ subscription (death) rate. The filter of each subscription is randomly generated. Therefore, the interval limits $a$ and $b$ are selected according to a probability density function $f^{s}(\cdot)$ which is identical but independent for both limits and all clients. In case that $a>b$, the interval limits are simply swapped.

Similarly to subscriptions, we can define the dynamic behavior of advertisements. We denote with $\lambda^{a}(B)^{-1}$ the mean interarrival time and with $\mu^{a}(B)^{-1}$ the mean lifetime of advertisement at broker $B$. The content of an advertisement is randomly chosen by using a probability density function $f^{a}(\cdot)$. All mentioned parameters including traffics, lifetimes and distributions can be set individually for each broker. This allows a flexible adjustment to real world scenarios.

Markovian Chains. As mentioned before, the process of creating and removing subscriptions resp. advertisements corresponds to a $M / M / \infty$ queueing system. According to Kleinrock [53], this continuous-time birth-death Markov chain is also known as responsive server. Each broker is modeled by independent Markovian chains for subscriptions and advertisements, where the birth rate $\lambda_{i}=\lambda$ does not depend on the current state of the chain. The death rate, which is the reciprocal value of the lifetime, depends on the state and is given by $\mu_{i}=i \cdot \mu$. The reason for the latter assumption is that each subscription/advertisement dies independently after an arbitrary amount of time generated by an exponential distribution. Figure 4.5(a) shows the resulting Markovian chain which is in state $k$ when the respective broker has $k$ simultaneously active subscriptions resp. advertisements.

This Markovian chain describes a dynamic process. This means, that at any fixed time the number of active subscriptions may range from 0 to any finite number. Since the modeling focus is on the equilibrium, we concentrate on the stationary distribution of the states. When the condition for a stationary distribution is met $(\lambda / \mu<\infty)$, we get according to Kleinrock [52] a Poisson distribution of the states:

$$
p_{k}=\frac{(\lambda / \mu)^{k}}{k !} \cdot e^{-\lambda / \mu}=\frac{N^{k}}{k !} \cdot e^{-N}
$$


The substitution $\lambda / \mu=N$ results from Little's Law [53]. In Figure 4.5(b), we exemplary show the stationary distribution for different values of $N$. The expected value as well as the variance of the Poisson distribution are both equal to $N$. Thus, the distributions move to the right and get wider for greater $N$. The Poisson distribution has an interesting property that is called additive property which also holds for the Gaussian distribution, the Gamma distribution, the Cauchy distribution and the $\chi^{2}$-distribution. The additive property for the Poisson distribution states that the sum of independent Poisson distributed random variables $X_{i} \sim \mathrm{P}\left(\lambda_{i}\right)$ is again Poisson distributed with a modified parameter $X_{1}+\cdots+X_{n} \sim \mathrm{P}\left(\lambda_{1}+\cdots+\lambda_{n}\right)$. This result is an outcome of the fact that the convolution of Poisson distributions is again a Poisson distribution. With the help of this property, we are able to subsume the behavior of multiple clients at a single broker by one accumulated Markovian chain. This subsumption can further be applied to describe the client behavior according to subscriptions in subtopologies ${ }^{2}$. For a subtopology rooted by the overlay link $B_{i} B_{j}$, the parameter of the Poisson distribution is equal to the expected number of active subscriptions/advertisements $N=N\left(B_{i} B_{j}\right)$. Thus, we can adapt Equation 4.1 as follows:

$$
\begin{aligned}
& q_{k}^{s}\left(B_{i} B_{j}\right)=\frac{N^{s}\left(B_{i} B_{j}\right)^{k}}{k !} \cdot e^{-N^{s}\left(B_{i} B_{j}\right)} \\
& q_{k}^{a}\left(B_{i} B_{j}\right)=\frac{N^{a}\left(B_{i} B_{j}\right)^{k}}{k !} \cdot e^{-N^{a}\left(B_{i} B_{j}\right)}
\end{aligned}
$$

The resulting formulas express the probability of having $k$ active subscriptions resp. advertisements in the subtopology $\mathcal{T}\left(B_{i} B_{j}\right)$.

\subsubsection{Routing Algorithms}

In our model, we consider five routing algorithms: flooding (FL), simple routing (SR), covering-based routing (CBR), identity-based routing (IBR), mergingbased routing (MBR). The behavior of FL and SR with notification resp. subscription flooding follows the described behavior in Section 2.4. In identity-based routing, we investigate a special case where a subscription is only propagated if there is no other active subscription available yet. This decision is made independently from the concrete attribute filters. This actually means that there is a single channel.

In the case of merging-based routing, we imperfectly merge all attribute filters into intervals with $\left[a_{\min }, b_{\max }\right]$, where $a_{\min }$ and $b_{\max }$ are the minimum $a_{i}$ and maximum $b_{i}$ of all active subscriptions in a subtopology. Thus, we investigate a version of merging-based routing that can be seen as an extension of identity-based routing. Beside these algorithms we also investigate advertisement-based routing (ABR). Following, we introduce formal definitions of matching as well as covering and overlapping. As a foundation of all routing algorithms the formalism simplifies further derivations.

Matching. Based on the set definition of notifications and filters (cf. Section 2.4.1), each matching can be reduced to the decision whether the notification is an element of the filter. Based on the introduced data and filter model,

\footnotetext{
${ }^{2} \mathrm{~A}$ formal definition of a subtopology comes later.
} 
we start with the definition for matching a single attribute. An attribute filter $I=[a, b]$ matches an attribute value $x$ (of a notification) iff $x$ is part of the interval $I$ :

$$
\begin{aligned}
\operatorname{matches}(I, x) & \Leftrightarrow x \in I \\
& \Leftrightarrow(a \leq x \leq b) \vee(a \geq x \geq b) \\
& \Leftrightarrow \neg[(a, b \leq x) \vee(a, b \geq x)]
\end{aligned}
$$

In Equation 4.4 we provide two expressions for the interval matching which are equivalent. The latter uses the comma as a short form of equal constraints on two variables. Since notifications and filters consist of only one attribute we define the matching of a filter $F=[I]$ with a notification $n=[x]$ as follows:

$$
\begin{aligned}
\operatorname{matches}(F, n) & \Leftrightarrow n \in F \\
& \Leftrightarrow \text { matches }(I, x)
\end{aligned}
$$

For routing purposes brokers manage notification routing tables $R T$. These routing tables are maintained by subscription that contain filters (cf. Section 2.3). In case of simple routing and covering-based routing, the routing table contains several routing entries with the same neighbor broker. As already mentioned, we call a list of filters that point to a neighbor broker filter list FList. An arbitrary notification filter list FList $=\left[F_{1}, \ldots, F_{k}\right]$ consists of $k$ filters. Such a filter list matches a notification $n=[x]$, iff at least one filter $F_{i}$ matches $n$. Formally,

$$
\begin{aligned}
\text { matches }(F \text { List }, n) & \Leftrightarrow n \in F \text { List } \\
& \Leftrightarrow \exists i: \text { matches }\left(F_{i}, n\right)
\end{aligned}
$$

Covering. In case of covering-based routing and merging-based routing, the covering relation of filters is essential to determine whether a subscription is forwarded or not. Analogously to the approach in matching, we start with the covering of single intervals. An interval $I_{i}=\left[a_{i}, b_{i}\right]$ covers $I_{j}=\left[a_{j}, b_{j}\right]$ if $I_{j}$ is a subset of $I_{i}$ which formally can be written as:

$$
\begin{aligned}
\operatorname{covers}\left(I_{i}, I_{j}\right) & \Leftrightarrow I_{i} \supseteq I_{j} \\
& \Leftrightarrow\left(a_{i} \leq a_{j}, b_{j} \leq b_{i}\right) \vee\left(b_{i} \leq a_{j}, b_{j} \leq a_{i}\right)
\end{aligned}
$$

We use the latter result to determine the covering relation of two filters. A filter $F_{i}=\left[I_{i}\right]$ covers another filter $F_{j}=\left[I_{j}\right]$ iff $I_{i}$ covers $I_{j}$ :

$$
\begin{aligned}
\operatorname{covers}\left(F_{i}, F_{j}\right) & \Leftrightarrow F_{i} \supseteq F_{j} \\
& \Leftrightarrow \operatorname{covers}\left(I_{i}, I_{j}\right)
\end{aligned}
$$

A filter list FList $=\left[F_{1}, \ldots, F_{k}\right]$ covers a filter $F$, iff at least one filter of FList covers $F$. Formally, 


$$
\begin{aligned}
\operatorname{covers}(F L i s t, F) & \Leftrightarrow F \text { List } \supseteq F \\
& \Leftrightarrow \exists i: \operatorname{covers}\left(F_{i}, F\right)
\end{aligned}
$$

For the subscription forwarding decision determined by a broker the inverse relation is used. If a filter $F$ is not covered by filter list $F$ List $=\left[F_{1}, \ldots, F_{k}\right]$ the subscription containing $F=[I]$ is forwarded. Thus,

$$
\begin{aligned}
\neg \operatorname{covers}(F \text { List }, F) \Leftrightarrow & \neg i: \operatorname{covers}\left(I_{i}, I\right) \\
& \Leftrightarrow \forall i: \neg \operatorname{covers}\left(I_{i}, I\right)
\end{aligned}
$$

Overlapping. If advertisement-based routing is used, a subscription is forwarded via an overlay link if there is an overlapping advertisement. The overlapping relation is true if the intersection of subscription and advertisement is not empty. Thus, overlapping is the inverse relation to disjunct relation. As before, we start with two intervals $I_{i}$ and $I_{j}$. Thus, we get,

$$
\begin{aligned}
\operatorname{averlaps}\left(I_{i}, I_{j}\right) & \Leftrightarrow\left(I_{i} \cap I_{j}\right) \neq \emptyset \\
& \Leftrightarrow \neg \operatorname{disjunct}\left(I_{i}, I_{j}\right) \\
& \Leftrightarrow \neg\left[\left(a_{j} \geq a_{i}, b_{i}\right) \vee\left(b_{j} \leq a_{i}, b_{i}\right)\right]
\end{aligned}
$$

Two filters $F_{i}=\left[I_{i}\right]$ and $F_{j}=\left[I_{j}\right]$ do overlap iff the attribute filter in $F_{i}$ overlaps the appropriate attribute filter in $F_{j}$.

$$
\begin{aligned}
\operatorname{overlaps}\left(F_{i}, F_{j}\right) \Leftrightarrow & \left(F_{i} \cap F_{j}\right)=\emptyset \\
& \Leftrightarrow \operatorname{overlaps}\left(I_{i}, I_{j}\right)
\end{aligned}
$$

In case of advertisement-based routing, there is a subscription routing table. The contained subscription filter lists are maintained by advertisements forwarding. A (subscription) filter list FList $=\left[F_{1}, \ldots, F_{k}\right]$ overlaps a filter $F=[I]$ (of a subscription), iff at least one filter of FList overlaps with $F$. Formally,

$$
\begin{aligned}
\operatorname{overlaps}(F \text { List }, F) \Leftrightarrow & (F \text { List } \cap F)=\emptyset \\
& \Leftrightarrow \exists i: \operatorname{overlaps}\left(F_{i}, F\right) \\
& \Leftrightarrow \exists i: \operatorname{overlaps}\left(I_{i}, I\right) \\
& \Leftrightarrow \neg \forall i: \operatorname{disjunct}\left(I_{i}, I\right)
\end{aligned}
$$

Later in this section we build on these definitions to derive various formulas.

\subsubsection{Broker Model}

Brokers have to handle incoming traffic from local clients as well as from neighbor brokers. In this thesis, brokers behave like a $M / M / 1$ queueing system. The service time distribution which is Markovian is determined by the capacity of the broker $c(B)$ and there is only one server resp. broker. A $M / M / 1$ broker 


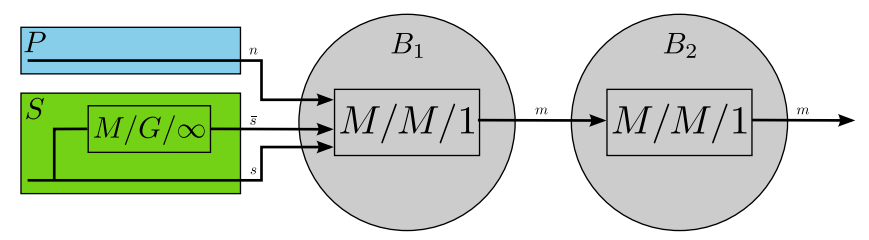

Figure 4.6: Queueing model of brokers including clients

requires that the arrival process, i.e., the receiving of messages is Markovian. To proof this fact, in Figure 4.6 we show a queueing model of brokers including two clients, a publisher and a subscriber. We have already pointed out that the interarrival times of notification $n$ and subscriptions $s$ are exponentially distributed. The first crucial point is the distribution of the interarrival time of unsubscriptions $\bar{s}$. According to Burke's theorem [17] in the equilibrium state the output process for $M / M / \infty$ is again a Poisson process with the same parameter as the input process, we can argue that the arrival of unsubscriptions is equally distributed as the arrival of subscriptions if the system is in steady state. Since the accumulation of Poisson distributed message flows is again Poisson distributed, broker $B_{1}$ receives a traffic which fits to the $M / M / 1$ model. Since Burke's theorem is also true for $M / M / 1$, the output of $B_{1}$ which is the input of broker $B_{2}$ is also Markovian. Thus, each queueing model used in this analysis does not change the type of distribution of the interarrival times of messages.

\subsubsection{Model for Physical Links and Routers}

Physical links and routers are also modeled as $M / M / 1$ queueing systems. The analysis that we just made for brokers is also true here. In this thesis, we do not differentiate between physical links and routers for the purpose of the analysis because both do only introduce a delay for messages. Thus, without limiting the generality, we only deal with physical links $\bar{l}$ in the rest of this thesis. Each physical link is described by its specific parameter $c(\bar{l})$ which is the capacity, i.e., the number of messages the link $\bar{l}$ can transmit per time unit. If a physical link is part of an overlay link $B_{i} B_{j}$, we denote this by $\bar{l} \in B_{i} B_{j}$.

\subsubsection{Auxiliary Measures}

For the derivation of the model, we define auxiliary measures that simplify the formulas. First, we provide definitions of important subsets of the set of all brokers $\mathcal{B}$. Despite the neighbor brokers these subsets are visualized in Figure 4.7. Second, we introduce accumulated measures based on broker-related measures such as the number of active subscriptions.

Neighbors. Let $\mathcal{N}\left(B_{i}\right)$ be the set of all neighbor brokers of a broker $B$. A broker $B_{j}$ belongs to $\mathcal{N}\left(B_{i}\right)$ if the overlay link $B_{i} B_{j}$ exists. For a single neighboring broker we often use the symbol $N$. 


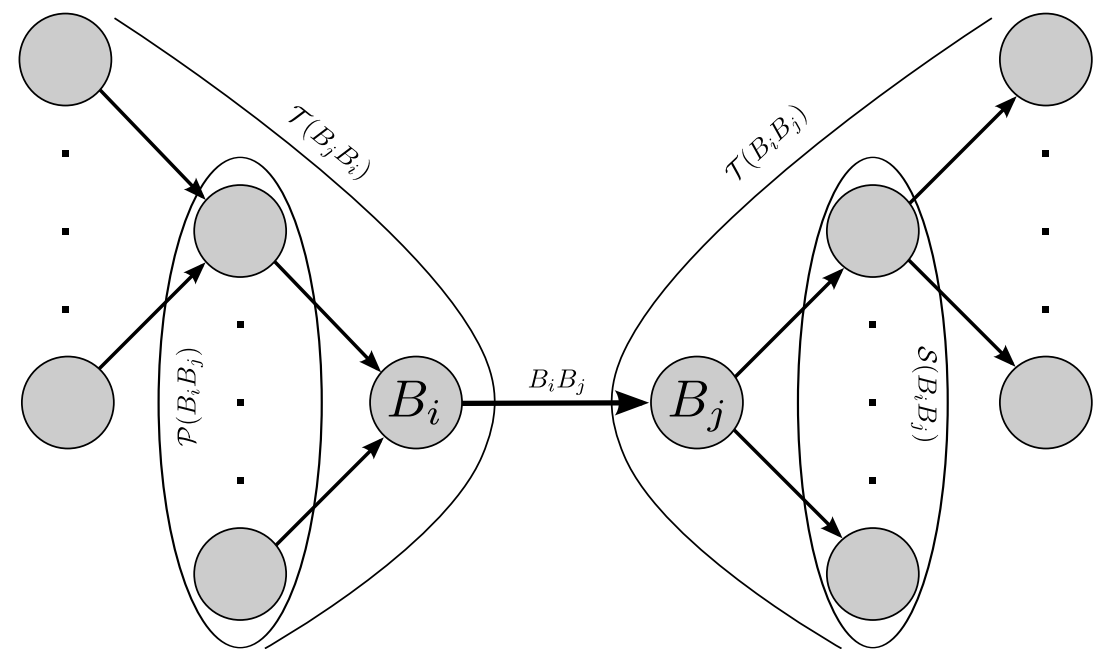

Figure 4.7: Auxiliary measures

Successors. Let $\mathcal{S}\left(B_{i} B_{j}\right)$ be the set of all successing brokers of the overlay link $B_{i} B_{j}$ formally defined by $\mathcal{S}\left(B_{i} B_{j}\right)=\mathcal{N}\left(B_{j}\right) \backslash\left\{B_{i}\right\}$. This set contains the potential destinations of messages that have been sent by broker $B_{i}$ and are received by $B_{j}$.

Predecessors. Let $\mathcal{P}\left(B_{i} B_{j}\right)$ be the set of all predecessing brokers of the overlay link $B_{i} B_{j}$ formally defined by $\mathcal{P}\left(B_{i} B_{j}\right)=\mathcal{N}\left(B_{i}\right) \backslash\left\{B_{j}\right\}$. This set contains the potential sources of messages that have been sent by broker $B_{i}$ and are received by $B_{j}$. This definition is the inverse definition to the latter one. Thus, it holds $\mathcal{S}\left(B_{i} B_{j}\right)=\mathcal{P}\left(B_{j} B_{i}\right)$.

Subtopology. Let $\mathcal{T}\left(B_{i} B_{j}\right)$ be the set of brokers of the subtopology rooted by overlay link $B_{i} B_{j}$. It is defined by $\mathcal{T}\left(B_{i} B_{j}\right)=\left\{B_{j}\right\} \cup \bigcup_{B_{k} \in \mathcal{S}\left(B_{j} B_{k}\right)} \mathcal{T}\left(B_{j} B_{k}\right)$ in a recursive way. A subtopology $\mathcal{T}\left(B_{i} B_{j}\right)$ contains all brokers that can be reached from broker $B_{i}$ via a neighbor broker $B_{j}$.

Accumulated Number of Active Subscriptions. $N^{s}\left(B_{i} B_{j}\right)$ is the number of active subscriptions of all local clients of all brokers of the subtopology $\mathcal{T}\left(B_{i} B_{j}\right) . N^{s}\left(B_{i} B_{j}\right)$ is equal to the number of filter entries at broker $B_{i}$ pointing to $B_{j}$ if simple routing is applied in the network. $N^{s}\left(B_{i} B_{j}\right)$ can be determined in a recursively as well as a non-recursively way.

$$
\begin{aligned}
N^{s}\left(B_{i} B_{j}\right) & =\sum_{B_{k} \in \mathcal{T}\left(B_{i} B_{j}\right)} x_{l}^{s}\left(B_{k}\right) \\
& =x_{l}^{s}\left(B_{j}\right)+\sum_{N \in \mathcal{S}\left(B_{i} B_{j}\right)} N^{s}\left(B_{j} N\right)
\end{aligned}
$$


Accumulated Number of Active Advertisements. Similar to $N^{s}\left(B_{i} B_{j}\right)$ the number of active advertisements in a subtopology $\mathcal{T}\left(B_{i} B_{j}\right)$ is defined as follows:

$$
\begin{aligned}
N^{a}\left(B_{i} B_{j}\right) & =\sum_{B_{k} \in \mathcal{T}\left(B_{i} B_{j}\right)} x_{l}^{a}\left(B_{k}\right) \\
& =x_{l}^{a}\left(B_{j}\right)+\sum_{N \in \mathcal{S}\left(B_{i} B_{j}\right)} N^{a}\left(B_{j} N\right)
\end{aligned}
$$

Accumulated Subscription Arrival Rate. $\lambda_{a c c}^{s}\left(B_{i} B_{j}\right)$ is the accumulated subscription issue rate generated by all local clients of all brokers of the subtopology $\mathcal{T}\left(B_{i} B_{j}\right) . \lambda_{a c c}^{s}\left(B_{i} B_{j}\right)$ is equal to the expected subscription traffic transmitted from broker $B_{j}$ to $B_{i}$ if simple routing is applied in the network.

$$
\begin{aligned}
\lambda_{a c c}^{s}\left(B_{i} B_{j}\right) & =\lambda^{s}\left(B_{j}\right)+\sum_{B_{k} \in \mathcal{T}\left(B_{i} B_{j}\right)} \lambda^{s}\left(B_{k}\right) \\
& =\lambda^{s}\left(B_{j}\right)+\sum_{N \in \mathcal{S}\left(B_{i} B_{j}\right)} \lambda_{a c c}^{s}\left(B_{j} N\right)
\end{aligned}
$$

Accumulated Publication Rate. $\omega_{a c c}\left(B_{i} B_{j}\right)$ is the accumulated publication rate generated by all local clients of all brokers of the subtopology $\mathcal{T}\left(B_{j} B_{i}\right)$. The rate $\omega_{a c c}\left(B_{i} B_{j}\right)$ is equal to the expected notification traffic transmitted from broker $B_{i}$ to $B_{j}$ if flooding is applied in the network.

$$
\begin{aligned}
\omega_{a c c}\left(B_{i} B_{j}\right) & =\omega\left(B_{i}\right)+\sum_{B_{k} \in \mathcal{T}\left(B_{j} B_{i}\right)} \omega\left(B_{k}\right) \\
& =\omega\left(B_{i}\right)+\sum_{N \in \mathcal{P}\left(B_{i} B_{j}\right)} \omega_{a c c}\left(N B_{i}\right)
\end{aligned}
$$

\subsubsection{Routing Table Size}

The routing table of each broker consists of local and remote routing entries. Following, we derive the expected routing table size for both types. We start with the notification routing table before going to the subscription routing table.

Local Notification Routing Size. The local routing table size of each broker can be easily calculated by applying Little's Law [53]. Here, the birth rate of subscriptions at a broker $\lambda^{s}(B)$ can be interpreted as the effective arrival rate while the lifetime $\mu^{s}(B)^{-1}$ equals the average time a customer spends in the system. Thus, we get:

$$
x_{l}^{s}(B)=\lambda^{s}(B) \cdot \mu^{s}(B)^{-1}
$$

This result does not depend on the applied routing algorithm since each algorithm has to perform local matching, and, therefore has to store all local routing entries. Nevertheless, $x_{l}^{s}(B)$ may be different between brokers due to individual rates and lifetimes. 
Remote Notification Routing Table Size of a Broker. The remote routing table of a broker $B$ contains all routing entries pointing to all its neighbors. Therefore, we can basically calculate the number of remote routing entries $X^{s}(B)$ by summing up the overlay link related number of routing entries $X^{s}(B N)$ which is calculated afterwards:

$$
X^{s}(B)=\sum_{N \in \mathcal{N}(B)} X^{s}(B N)
$$

Remote Notification Routing Size of an Overlay Link. Remote routing entries of an overlay link $B_{i} B_{j}$ are those routing entries of the routing table $R T_{B_{i}}$ which point to broker $B_{j}$. This subset of the routing table equals to the given definition of filter list FList in Section 4.4.2. Let $x_{A L}^{s}(k)$ be the expected number of remote routing entries if there are $k$ active subscriptions in the subtopology $\mathcal{T}\left(B_{i} B_{j}\right)$. Then, we can determine the expected value in case of the Markovian subscription behavior (cf. Equation 4.2) as follows:

$$
X_{A L}^{s}\left(B_{i} B_{j}\right)=\sum_{k=0}^{\infty} q_{k}^{s}\left(B_{i} B_{j}\right) \cdot x_{A L}^{s}(k)
$$

The quantities $x_{A L}^{s}(k)$ and $X_{A L}^{s}\left(B_{i} B_{j}\right)$ depend on the applied routing algorithm (AL). Thus, we subsequently discuss its instances for all considered algorithms.

Flooding. When flooding is used, the number of remote routing entries is always zero and does not depend on the number of active subscriptions. Therefore, it holds $x_{F L}^{s}(k)=0$ for all $k$. Due to the identical value for all $k$, the expected number of remote entries is independently from the distribution of the number of subscriptions. Thus, we get:

$$
X_{F L}^{s}\left(B_{i} B_{j}\right)=0
$$

Later, in Chapter 6 flooding is realized by establishing a True-Filter which instead would lead to $X_{F L}^{s}\left(B_{i} B_{j}\right)=1$. Since the matching costs are often directly coupled to the routing table, we prefer the former calculation which may lead to zero matching costs which is closer to the real implementation of flooding.

Simple Routing. In simple routing, each active subscription eventually generates a routing entry on each broker. Therefore, it holds that $x_{S R}^{s}(k)=k$. A substitution into Equation 4.20 requires mathematical conversions to get the following simple result for the expected number of remote entries: 


$$
\begin{aligned}
X_{S R}^{s}\left(B_{i} B_{j}\right) & =\sum_{k=0}^{\infty} \frac{N^{s}\left(B_{i} B_{j}\right)^{k}}{k !} \cdot e^{-N^{s}\left(B_{i} B_{j}\right)} \cdot k \\
& =e^{-N^{s}\left(B_{i} B_{j}\right)} \cdot N^{s}\left(B_{i} B_{j}\right) \cdot \sum_{k=0}^{\infty} \frac{N^{s}\left(B_{i} B_{j}\right)^{k-1}}{(k-1) !} \\
& =e^{-N^{s}\left(B_{i} B_{j}\right)} \cdot N^{s}\left(B_{i} B_{j}\right) \cdot e^{N^{s}\left(B_{i} B_{j}\right)} \\
& =N^{s}\left(B_{i} B_{j}\right)
\end{aligned}
$$

As expected for SR the expected number of remote routing entries grows linearly with the number of active subscription in the related subtopology.

Identity-based and Merging-based Routing. In identity-based routing as well as merging-based routing, a broker $B_{i}$ has a single remote routing entry pointing to its neighbor broker $B_{j}$ if there is at least one active subscription in the subtopology $\mathcal{T}\left(B_{i} B_{j}\right)$. Thus, $x_{I B R}^{s}(0)=0$ and $x_{I B R}^{s}(k)=1$ for $k>0$. After the substitution into Equation 4.20 and conversion we get:

$$
\begin{aligned}
X_{I B R}^{s}\left(B_{i} B_{j}\right) & =\sum_{k=1}^{\infty} \frac{N^{s}\left(B_{i} B_{j}\right)^{k}}{k !} \cdot e^{-N^{s}\left(B_{i} B_{j}\right)} \\
& =e^{-N^{s}\left(B_{i} B_{j}\right)} \cdot \sum_{k=1}^{\infty} \frac{N^{s}\left(B_{i} B_{j}\right)^{k}}{k !} \\
& =e^{-N^{s}\left(B_{i} B_{j}\right)} \cdot\left[e^{-N^{s}\left(B_{i} B_{j}\right)}-1\right] \\
& =1-e^{-N^{s}\left(B_{i} B_{j}\right)}
\end{aligned}
$$

This means that the expected number of subscriptions asymptotically grows from 0 to the upper bound of 1 for IBR and MBR.

Covering-based Routing. In covering-based routing, each remote routing entry for a neighbor broker $B_{j}$ corresponds to a subscription existing in the subtopology $\mathcal{T}\left(B_{i} B_{j}\right)$ that is not covered by any other subscription existing in this subtopology. Let $p^{\neg \operatorname{cov}}(k)$ be the probability that a subscription is not covered by any of $k$ other subscriptions. Then, when $k$ subscriptions are active, each single subscription must not be covered by $k-1$ other subscriptions leading to $x_{C B R}^{s}(k)=k \cdot p^{\urcorner c o v}(k-1)$. For undisturbed readability the derivation of $p^{\neg \operatorname{cov}}(k)$ is postponed to Section 4.4.7. With this result we can substitute $x_{C B R}^{s}(k)$ in Equation 4.20 which unfortunately does not simplify the equation:

$$
X_{C B R}^{s}\left(B_{i} B_{j}\right)=\sum_{k=0}^{\infty} q_{k}^{s}\left(B_{i} B_{j}\right) \cdot k \cdot p^{\neg c o v}(k-1)
$$

To quantitatively illustrate the formulas, we compare the algorithms in Figure 4.8. In further sections we keep the color for the algorithms, such as black for FL resp. red for SR for example. In this plot we show the expected number of remote routing entries $X_{A L}^{s}\left(N^{s}\right)$ on one overlay link and vary the number 


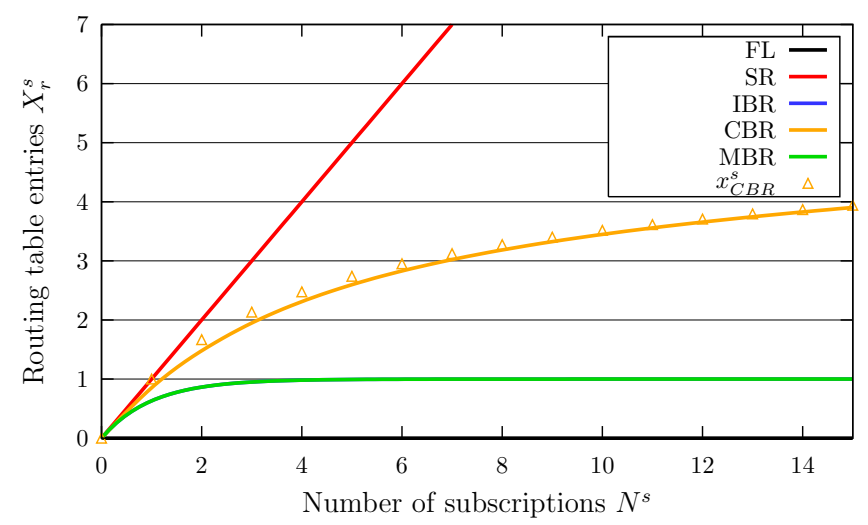

Figure 4.8: Remote routing entries

of active subscription $N^{s}$ in the related subtopology from 0 to 15 . The plot shows that for all values of $X^{s}$ we can order the routing algorithms as follows: $0=X_{F L}^{s} \leq X_{I B R}^{s}=X_{M B R}^{s} \leq X_{C B R}^{s} \leq X_{S R}^{s}=N^{s}$. While flooding has no routing entries, identity-based routing and merging-based routing do converge asymptotically to 1 routing entry. While simple routing linearly grows with the number of active subscriptions due to its subscription flooding behavior, covering-based routing diverges in a square root like function. The figure also shows the difference between expected value and $x_{C B R}^{s}$ which only exists for integers. These formulas are the first performance measures of the model of the publish/subscribe system. By summing up the remote routing table entries for all links one can determine the size of the remote notification routing table $X_{A L}^{s}(B)$ for each broker. Together with the local routing table size $x_{l}^{s}(B)$, it is a good cost measure and allows to determine the RAM consumption for example.

Advertisement-based Routing. Similarly to notification routing, we can determine routing tables sizes for the routing of subscription in advertisementbased routing. The number of local subscription routing table entries, the remote subscription routing table size of brokers resp. overlay links are determined as follows:

$$
\begin{aligned}
& x_{l}^{a}(B)=\lambda^{a}(B) \cdot \mu^{a}(B)^{-1} \\
& X^{a}(B)=\sum_{N \in \mathcal{N}(B)} X^{a}(B N) \\
& X_{A L}^{a}\left(B_{i} B_{j}\right)=\sum_{k=0}^{\infty} q_{k}^{a}\left(B_{i} B_{j}\right) \cdot x_{A L}^{a}(k)
\end{aligned}
$$

Since on advertisement routing layer the same algorithms as for subscription routing can be used, the formulas for $x_{A L}^{a}(k)$ are practically the same. 


\subsubsection{Forwarding Probabilities of Messages}

The central quantities of the derivation of the stochastic system model are the forwarding probabilities of messages $P^{x}$. They exist for all messages types $x$, i.e., for notifications $P^{n}$, (un)subscriptions $P^{s}$ and (un)advertisements $P^{a}$ and depend on the selected routing algorithm. A forwarding probability is always related to an overlay link $B_{i} B_{j}$ and defines the fraction of the messages that are (potentially) received by $B_{i}$ and forwarded to broker $B_{j}$. Generally, we distinguish between two kinds of probabilities for different message types $x$ : the normal probabilities $P^{x}\left(B_{i} B_{j}\right)$ and the effective probabilities $\hat{P}^{x}\left(B_{i} B_{j}\right)$. The normal forwarding probability $P^{x}\left(B_{i} B_{j}\right)$ expresses the probability that a message of type $x$ issued in $\mathcal{T}\left(B_{j} B_{i}\right)$ is forwarded to $B_{j}$. Here a potential prefiltering of messages is left out. Instead it assumes that all messages of type $x$ do reach $B_{i}$. On the other hand, $\hat{P}^{x}\left(B_{i} B_{j}\right)$ depends on the prefiltering in $\mathcal{T}\left(B_{j} B_{i}\right)$. Therefore, this probability is the ratio between the traffic on $B_{i} B_{j}$ and the effective incoming traffic of $B_{i}$. It always holds $\hat{P}^{x}\left(B_{i} B_{j}\right) \geq P^{x}\left(B_{i} B_{j}\right)$. Following we only use normal forwarding probabilities, the effective probabilities are used in Section 6.4. There, also a formula for converting one to the other is given.

\section{Forwarding Probability for Notifications}

The forwarding probability $P^{n}\left(B_{i} B_{j}\right)$ is the probability that an incoming notification on broker $B_{i}$ is forwarded to broker $B_{j}$. This value is determined by the filters of $B_{i}$ pointing to $B_{j}$ which depend on the number of active subscriptions in subtopology $\mathcal{T}\left(B_{i} B_{j}\right)$ and the routing algorithm applied on link $B_{i} B_{j}$. Since all routing algorithms are correct in the sense of Definition 2.4.2, the lower bound of $P^{n}$ is determined by the number of notifications for which a subscriber exist in the subtopology. Imperfect algorithms, such as flooding or merging-based routing, tend to have greater values. Similar to Equation 4.20, we express $P^{n}\left(B_{i} B_{j}\right)$ as an expected value of $p^{n}(k)$, where $p^{n}(k)$ is the probability that a notification is forwarded if there are $k$ active subscriptions in the respective subtopology.

$$
P_{A L}^{n}\left(B_{i} B_{j}\right)=\sum_{k=0}^{\infty} q_{k}^{s}\left(B_{i} B_{j}\right) \cdot p_{A L}^{n}(k)
$$

Due to the dependency of the forwarding probability on the routing algorithm, we again add the subscript AL. Both, $p^{n}(k)$ and $P^{n}\left(B_{i} B_{j}\right)$ are monotonic increasing function in $k$ resp. $N^{s}\left(B_{i} B_{j}\right)$ because an increased number of subscriptions means an increased interest and therefore, and increased probability that a published notification has to be forwarded. Following, we derive $P_{A L}^{n}\left(B_{i} B_{j}\right)$ for all important routing algorithms.

Flooding. When flooding is used, all incoming notifications are directly forwarded to all neighbor brokers. Therefore, the probability is $p_{F L}^{n}(k)=1$ for all $k \geq 0$. Substituted in Equation 4.28 we get:

$$
P_{F L}^{n}\left(B_{i} B_{j}\right)=1
$$


Identity-based Routing. The identity-based routing is based on channels. If there is at least one subscription (for a channel), all notifications are forwarded by the broker. Thus, we have $p_{I B R}^{n}(0)=0$ and $p_{I B R}^{n}(k)=1$ for $k>0$ implying after substitution in Equation 4.28 (cf. Section 4.4.6):

$$
P_{I B R}^{n}\left(B_{i} B_{j}\right)=1-e^{-N^{s}\left(B_{i} B_{j}\right)}
$$

Simple and Covering-based Routing. To determine the forwarding probabilities of simple routing and covering-based routing, we have to determine the probabilities of predicate logic formulas as in the definitions of matching, covering and merging introduced in Section 4.4.2. All formulas contain quantifier such as $\forall$ and $\exists$ and independent predicates $X_{i}$ which are either true or false. Following we show the calculus for the determination of the overall probability if the probabilities for the predicates $p_{i}$ are given:

1. $\forall X_{i}:\left(X_{i}=\right.$ true $) \Rightarrow p_{1} \cdot \ldots \cdot p_{n}$

2. $\forall X_{i}:\left(X_{i}=\right.$ false $) \Rightarrow\left(1-p_{1}\right) \cdot \ldots \cdot\left(1-p_{n}\right)$

3. $\exists X_{i}:\left(X_{i}=\right.$ true $) \Leftrightarrow \neg \forall X_{i}:\left(X_{i}=\right.$ false $) \Rightarrow 1-\left(1-p_{1}\right) \cdot \ldots \cdot\left(1-p_{n}\right)$

4. $\exists X_{i}:\left(X_{i}=\right.$ false $) \Leftrightarrow \neg \forall X_{i}:\left(X_{i}=\right.$ true $) \Rightarrow 1-p_{1} \cdot \ldots \cdot p_{n}$

With the help of this calculus the reader easily can follow the derivation of the formulas. In simple and covering-based routing only notifications are delivered for which a subscriber exists that is interested in it. Nevertheless, there may occur false positives (FP) notification because a subscriber may unsubscribe while a notification is on the way. This effect can be skipped because such situations are relatively rare. According to Equation 4.6, a onedimensional filter list matches a notification if there is at least one filter that matches - matches (FList, $n) \Leftrightarrow \exists i$ : matches $\left(F_{i}, n\right)$. To better determine the matching probabilities, we mark random quantities with a bar on top. When $P\left(\right.$ matches $\left.\left(\bar{F}_{i}, n\right)\right)$ is the probability that a randomly chosen filter matches a notification with an arbitrary but fixed value $x$ we can derive the formula for the probability that an arbitrary notification is matched by a filter list consisting of $k$ randomly chosen filters:

$$
P(\operatorname{matches}(F \bar{L} i s t, n))=1-[1-P(\operatorname{matches}(\bar{F}, x))]^{k}
$$

The simple multiplication of the probabilities requires independent random variables between filters in the filter list. Therefore, we left out index $i$. The probability that matches $(\bar{F}, x)$ is the probability that for an arbitrary but fixed point $x$ both randomly chosen interval limits are smaller than $x$ or greater than $x$. Both interval limits are chosen according to $f^{s}(\cdot)$.

$$
\begin{aligned}
P(\operatorname{matches}(\bar{F}, x)) & =1-[P(\bar{a} \leq x) \cdot P(\bar{b} \leq x)+P(\bar{a} \geq x) \cdot P(\bar{b} \geq x)] \\
& =1-\left[F^{s}(x)\right]^{2}-\left[1-F^{s}(x)\right]^{2} \\
& =2 \cdot F^{s}(x) \cdot\left(1-F^{s}(x)\right)
\end{aligned}
$$




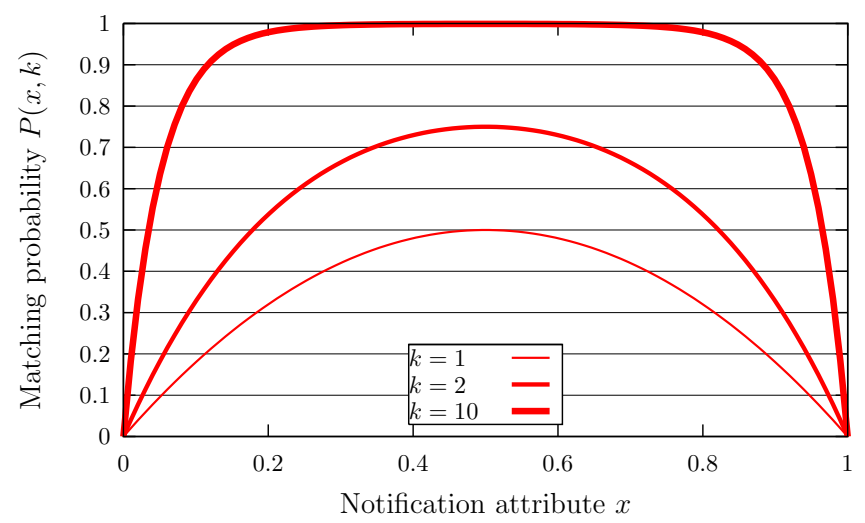

Figure 4.9: Matching probability for SR/CBR: $1-(1-2 x(1-x))^{k}$

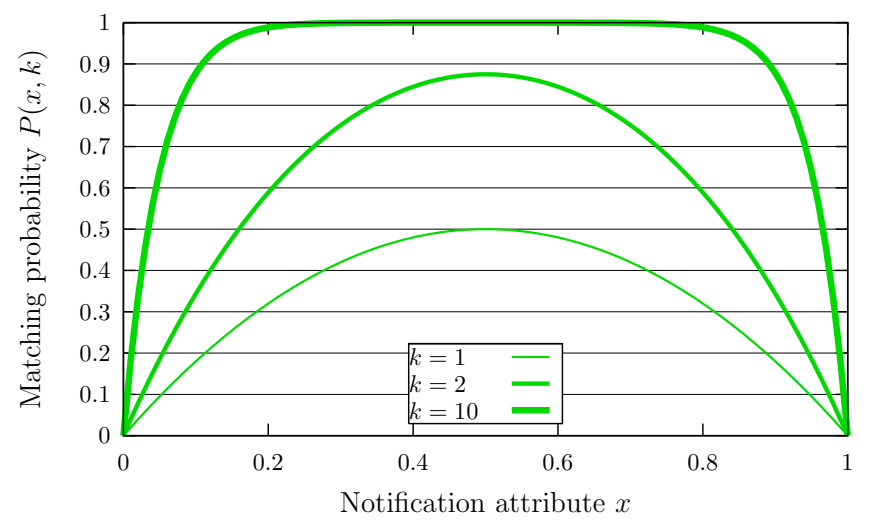

Figure 4.10: Matching probability for MBR: $1-\left(x^{2 k}+(1-x)^{2 k}\right)$

In the latter equation $F^{s}(x)$ is the cumulative distribution function defined as $F^{s}(x)=\int_{-\infty}^{x} f^{s}\left(x^{\prime}\right) \mathrm{d} x^{\prime}$ according to standard probability theory. The resulting equation allows us to concretize Equation 4.31:

$$
\begin{aligned}
P_{C B R}^{\text {match }}(x, k) & =P(\text { matches }(F \overline{L i s t}, n)) \\
& =1-\left[1-2 \cdot F^{s}(x) \cdot\left(1-F^{s}(x)\right)\right]^{k}
\end{aligned}
$$

$P($ matches $(F \bar{L} i s t, n))$ is the probability that a certain notification with the attribute $x$ is matched (covered) by at least one of $k$ subscriptions. It depends on the variable $k$ which is the number of filters and on $x$ which is the notification. Hence, we shortly write $P_{C B R}^{\text {match }}(x, k)$. In Figures 4.9, we plotted the probability for a simplified scenario with an interval limit distribution of $F^{s}(x)=x$ within the interval $[0,1]$. A general property of the matching probability is that the closer $x$ is to one of the interval limits, the smaller $P_{C B R}^{\text {match }}(x, k)$ is. Due to the symmetry, the maximum is reached in the middle of the interval at $x=0.5$. For increasing $k$, the probability for all notifications is increased. For $k \rightarrow \infty$ the probability $P_{C B R}^{\text {match }}(x, k)$ tends to be 1 for all notifications. 
This interim result $P_{C B R}^{\text {match }}(x, k)$ is itself a random variable based on the state space of the attribute value $x$ of the notification. The expected matching probability of a notification whose attribute value is distributed according to $f^{n}(x)$ when $k$ subscriptions are active is then:

$$
\begin{aligned}
p^{\text {match }}(k) & =E\left[P_{C B R}^{\text {match }}(x, k)\right] \\
& =\int_{-\infty}^{\infty} f^{n}(x) \cdot P_{C B R}^{\text {match }}(x, k) \mathrm{d} x \\
& =\int_{-\infty}^{\infty} f^{n}(x) \cdot\left(1-\left[1-2 \cdot F^{s}(x) \cdot\left(1-F^{s}(x)\right)\right]^{k}\right) \mathrm{d} x
\end{aligned}
$$

In case of a uniform distribution between 0 and 1 for the notification attribute values and filter interval limits, i.e., for $f^{s}(x)=f^{n}(x)=1$, we get a simplified formula:

$$
p^{\text {match }}(k)=\int_{0}^{1} 1-[1-2 \cdot x(1-x)]^{k} \mathrm{~d} x
$$

Based on the matching probability, we are able to determine the forwarding probability for notifications when covering-based routing is applied. Since a further simplification is not possible, we can determine the expected notification forwarding probability based on Equation 4.28 by this infinite sum:

$$
P_{C B R}^{n}\left(B_{i} B_{j}\right)=\sum_{k=0}^{\infty} q_{k}^{s}\left(B_{i} B_{j}\right) \cdot p^{\text {match }}(k)
$$

Merging-based Routing. In merging-based routing, a notification with the attribute value $x$ is propagated from broker $B_{i}$ to $B_{j}$ if there is (i) a subscription $\left[a_{m}, b_{m}\right]$ in the subtopology $\mathcal{T}\left(B_{i} B_{j}\right)$ with $a_{m} \leq x$ and (ii) a subscription $\left[a_{n}, b_{n}\right]$ with $x \leq b_{n}$. These conditions can also be satisfied by a single subscription $(m=n)$. Obviously, it holds that $p^{n}(k)=0$ for $k=0$. For $k>0$ subscriptions, a notification is not matched if all $2 k$ interval limits are either lower or greater than $x$ leading to $F^{s}(x)^{2 k}+\left[1-F^{s}(x)\right]^{2 k}$. Thus, we get:

$$
P(\text { matches }(F \text { List }, n))=1-\left(\left[F^{s}(x)\right]^{2 k}+\left[1-F^{s}(x)\right]^{2 k}\right)=P_{M B R}^{\text {match }}(x, k)
$$

Please note that FList is a virtual filter list consisting of all active filters within the related subtopology. In reality, the routing table does contain at most one entry per destination. In Figure 4.10, the matching probability $P_{M B R}^{m a t c h}(x, k)$ is plotted for uniformly distributed $f^{s}(x)=1$ interval limits between 0 and 1 . The results are similar to the SR/MBR case. Especially, for $k=1$ where no merging is possible the graphs are identical since $1-(1-2 x(1-x))=1-\left(x^{2}+(1-x)^{2}\right)$. For greater $k$, the matching probabilities of MBR are greater than of SR/CBR for all $x$ due to the forwarding of false positive notifications. The expected matching probability can be calculated by an integration, then:

$$
\begin{aligned}
p^{\text {match }}(k) & =E\left[P_{M B R}^{\text {match }}(\text { matches }(\text { F List }, n))\right] \\
p_{M B R}^{n}(k) & =\int_{-\infty}^{\infty} f^{n}(x) \cdot\left(1-\left[F^{s}(x)^{2 k}+\left(1-F^{s}(x)\right)^{2 k}\right]\right) \mathrm{d} x
\end{aligned}
$$




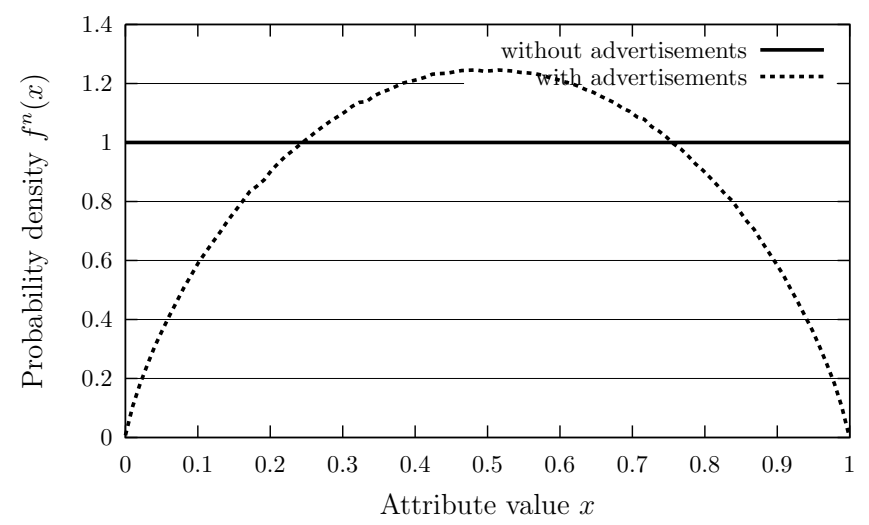

Figure 4.11: Distribution of notifications

Thus, we have the forwarding probability $p_{M B R}^{n}(k)$ which is the probability that a notification is matched by one out of $k$ filters. In case of a uniform distribution of the attributes of the notifications and filters, we can simplify the latter equation and solve the integral as follows:

$$
\begin{aligned}
p_{M B R}^{n}(k) & =\int_{-\infty}^{\infty} 1-\left[x^{2 k}+(1-x)^{2 k}\right] \mathrm{d} x \\
& =1-\left[\frac{x^{2 k+1}-(1-x)^{2 k+1}}{2 k+1}\right]_{0}^{1} \\
& =\frac{2 k-1}{2 k+1}
\end{aligned}
$$

As already mentioned, the results for $p_{M B R}^{n}(1)$ and $p_{S R}^{n}(1)$ are equal with the value $\frac{1}{3}$. Finally, we are now able to determine the expected forwarding probability considering a fluctuating number of active subscriptions based on Equation 4.28:

$$
P_{M B R}^{n}\left(B_{i} B_{j}\right)=\sum_{k=0}^{\infty} q_{k}^{s}\left(B_{i} B_{j}\right) \cdot \frac{2 k-1}{2 k+1}
$$

Advertisement-based Routing. When advertisement-based routing is used, advertising and publishing are coupled in opposite to subscribing and publishing which are totally orthogonal. In Figure 4.11 we see how the notification density changes with and without the usage of advertisements. Without advertisements, we uniformly set the attribute of notifications within the interval $[0,1]$. When advertisements are used, published notifications must be located within the range of active advertisement filters. Therefore, we first randomly generate an advertisement $[a, b]$. Within this interval, we choose the notification with a uniform distribution $\left(f(x)=\frac{1}{|b-a|}\right)$. The resulting global distribution $f^{n}(x)$ is depicted by the dashed curve in the figure which has been generated by ten simulation runs. Notifications close to the limits 0 and 1 have a decreased probability since advertisement intervals are more likely to cover the center. The modified 


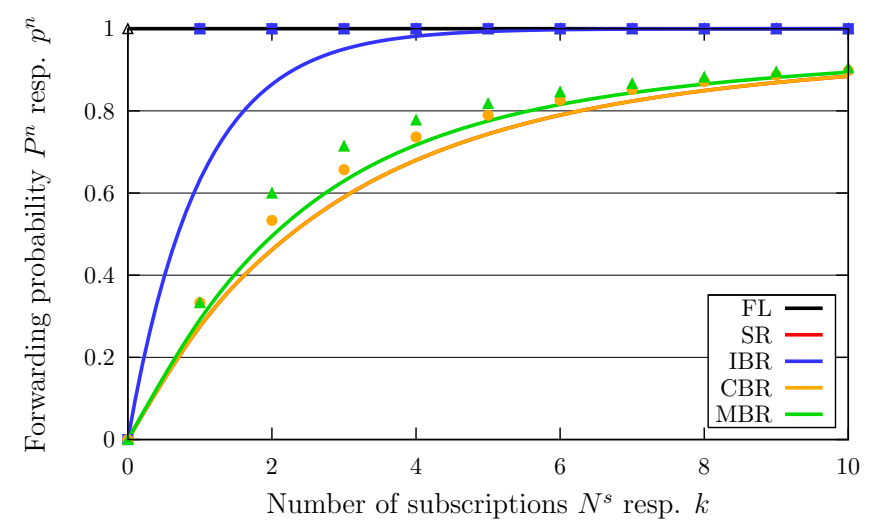

Figure 4.12: Notifications probabilities

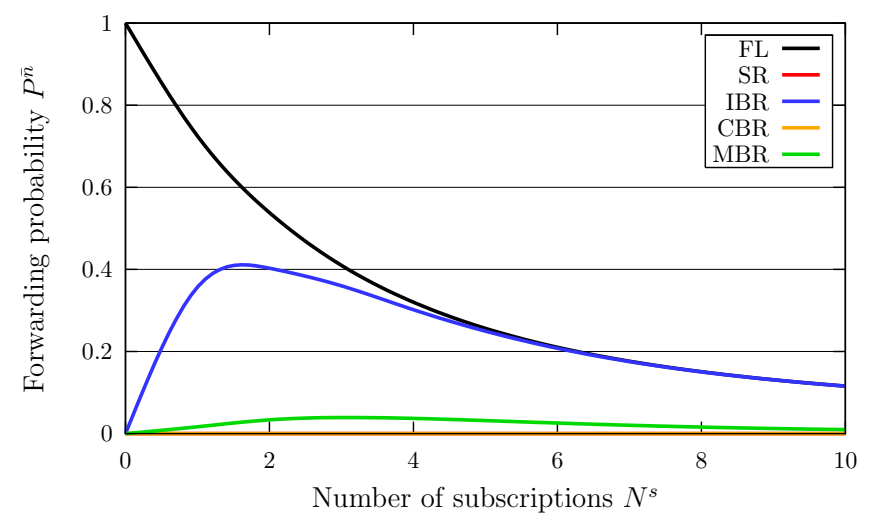

Figure 4.13: False positive probabilities

distribution $f^{n}(x)$ can be used in formula $P^{n}$, but it makes a comparison more difficult.

Comparison. In Figure 4.12 we show the forwarding probabilities for all considered routing algorithms despite advertisement-based routing. Attributes and filters are uniformly distributed between 0 and 1 . For information we added $p^{n}(k)$ which is a discrete measure and, thus, it is plotted by points instead of lines. $P^{n}\left(B_{i} B_{j}\right)$ is a continuous function which depends on the expected number of subscriptions in the observed subtopology $N^{s}\left(B_{i} B_{j}\right)$ which determines the Poisson distribution contained in $q_{k}^{s}\left(B_{i} B_{j}\right)$. As expected, the notification forwarding probability monotonically increases in the number of subscriptions and converges to $100 \%$. The lower bound for such a probability curve is given by SR resp. CBR since they only deliver interesting messages. Lower probabilities would hurt the correctness definition. This lower bound does hold for all imaginable algorithms, not only the ones we investigate here. The upper bound is given by the maximal value a probability can have $(100 \%)$, which is realized by flooding which simply forwards all incoming notifications. In between these bounds 
we find MBR and IBR which are instances of algorithms that generalize the interests (subscriptions) to reduce the routing table size (cf. Section 4.4.6) and reduce the generated control traffic (cf. Section 4.4.7). This advantage is at the expense of more false positive notifications. The notification forwarding probabilities $p^{n}$ are ordered as follows: $1=p_{F L}^{n} \geq p_{I B R}^{n} \geq p_{M B R}^{n} \geq p_{C B R}^{n}=p_{S R}^{n} \geq 0$. The depicted values of $p^{n}$ in Figure 4.12 tend to converge to $P^{n}$ for greater values, a fact which is utilized in the efficient calculation of the model explained in Chapter 5. The fact that at only $N^{s}=10$ (which is a rather small value) the probabilities are approximately 1 is caused by the restriction on a single attribute. In Section 4.6, we will show how this probability drops if more attributes for notifications and filters are allowed.

False Positive Notifications. An important byproduct of the derived formulas is that we are able to calculate the probability that a notification is unnecessarily forwarded. With this information it is easy to determine the unwanted notification traffic (cf. Section 4.4.8). The probability for such false positive notification deliveries on an arbitrary overlay link is the difference between the forwarding probability of the applied routing algorithm AL and a perfect routing algorithm like SR or CBR. Thus, we get the false positive forwarding probability for an arbitrary routing algorithm:

$$
\begin{aligned}
P_{A L}^{\bar{n}}\left(B_{i} B_{j}\right) & =P_{A L}^{n}\left(B_{i} B_{j}\right)-P_{A L}^{n}\left(B_{i} B_{j}\right) \\
& =\sum_{k=0}^{\infty} q_{k}^{s}\left(B_{i} B_{j}\right) \cdot\left[p_{A L}^{n}(k)-p_{S R}^{n}(k)\right]
\end{aligned}
$$

In Figure 4.13 the results for all routing algorithms despite advertisement-based routing are depicted. Obviously, for SR and CBR the false positive probabilities are $0 \%$. In case of flooding the false positives monotonically decrease with increased number of subscriptions $N^{s}$ which can be explained by the increasing interest if more and more subscribers appear. With IBR and MBR the number of FPs increases to a maximum of $40 \%$ at around $N^{s}=1.5$ resp. $5 \%$ at $N^{s}=3$ before converging to 0 then. Since IBR is actually flooding if there is at least 1 subscription, the graph of IBR tends asymptotically tends to FL.

\section{Forwarding Probability for Subscriptions}

The forwarding probability $P^{s}\left(B_{i} B_{j}\right)$ is the probability that an incoming subscription at broker $B_{j}$ is forwarded to broker $B_{i}$. This inverted definition compared to $P^{n}\left(B_{i} B_{j}\right)$ has a systematic reason that will become more clearly when we calculate the traffic in Section 4.4.8. To be precise, we would need to determine two forwarding probabilities $P^{s}$ resp. $P^{\bar{s}}$ in this section, but for all considered routing algorithms the decision whether a subscription is forwarded or not equals the decision of an unsubscription. Thus, we only focus on subscription forwarding in this section. For the calculation of traffic we consider the fact that each subscription has a corresponding unsubscription message by doubling the birth rate.

The quantity $p^{s}(k)$ is the probability that a subscription is forwarded when there are currently $k$ other subscriptions active in the subtopology $\mathcal{T}\left(B_{j} B_{i}\right)$. It also depends on the selected algorithm AL. Similar to Equations 4.20 and 4.28, 
we now determine the expected subscription forwarding probability $P^{s}\left(B_{i} B_{j}\right)$ for a Poisson distributed number of subscriptions $q_{k}^{s}\left(B_{i} B_{j}\right)$ and $p^{s}(k)$ :

$$
P_{A L}^{s}\left(B_{i} B_{j}\right)=\sum_{k=0}^{\infty} q_{k}^{s}\left(B_{i} B_{j}\right) \cdot p_{A L}^{s}(k)
$$

Both $p^{s}(k)$ and $P^{s}\left(B_{i} B_{j}\right)$ are monotonic decreasing functions in the number of subscriptions $k$ resp. $N^{s}\left(B_{j} B_{i}\right)$. The reason for that are the optimizations of the routing algorithms that try to avoid redundant subscriptions. Therefore, an increased number of subscriptions decreases the probability that a newly issued subscription adds new interest.

Flooding. When flooding is used on a link, a subscription is never propagated. Thus, $p_{F L}^{s}(k)=0$ for $k \geq 0$. After a substitution into Equation 4.41 we get:

$$
P_{F L}^{s}\left(B_{i} B_{j}\right)=0
$$

Simple Routing. With simple routing, a subscription issued or revoked is always propagated as a control message on link $B_{i} B_{j}$ regardless how many other subscriptions are active in the subtopology $\mathcal{T}\left(B_{i} B_{j}\right)$. Thus, $p_{S R}^{s}(k)=1$ for $k \geq 0$. After a substitution in Equation 4.41 it follows:

$$
P_{S R}^{s}\left(B_{i} B_{j}\right)=1
$$

Identity-based Routing. With identity-based routing, a subscription issued or revoked leads to a control message propagated by broker $B_{j}$ to its neighbor broker $B_{i}$ if there is no other subscription in the subtopology $\mathcal{T}\left(B_{i} B_{j}\right)$. Hence, it is $p_{I B R}^{s}(0)=1$ and $p_{I B R}^{s}(k)=0$ for $k>0$, implying after a substitution into Equation 4.41:

$$
\begin{aligned}
P_{I B R}^{s}\left(B_{i} B_{j}\right) & =\sum_{k=0}^{0} \frac{N^{s}\left(B_{i} B_{j}\right)^{k}}{k !} \cdot e^{-N^{s}\left(B_{i} B_{j}\right)} \\
& =e^{-N^{s}\left(B_{i} B_{j}\right)}
\end{aligned}
$$

Covering-based Routing. In covering-based routing, only those subscriptions are delivered for which no covering filter in $\mathcal{T}\left(B_{i} B_{j}\right)$ exists. According to Equation 4.10 a filter list $F$ List does not cover a filter $F$ if all filters of the list do not cover $F: \neg \operatorname{covers}(F$ List,$F) \Leftrightarrow \forall i: \neg \operatorname{covers}\left(I_{i}, J\right)$. Thus, we can derive a formula for the probability that an arbitrary subscription is not covered by a filter list consisting of $k$ randomly chosen filters:

$$
P(\neg \operatorname{covers}(F \bar{L} i s t, F))=[1-P(\operatorname{covers}(\bar{I}, J))]^{k}
$$

$P(\operatorname{covers}(\bar{I}, J))$ is the probability that an arbitrary but fixed interval $J$ with its limits $a$ and $b$ is covered by a randomly generated interval $\bar{I}$ whose limits $\bar{a}^{\prime}$ and $\bar{b}^{\prime}$ are generated by the probability density function $f^{s}(\cdot)$. Considering the fact that for the limits it must hold that $\left(\bar{a}^{\prime} \leq a, b \leq \bar{b}^{\prime}\right) \vee\left(\bar{b}^{\prime} \leq a, b \leq \bar{a}^{\prime}\right)$, the probability can be calculated as follows: 


$$
\begin{aligned}
P(\operatorname{covers}(\bar{I}, J)) & =P\left(\bar{a}_{i} \leq a\right) \cdot P\left(\bar{b}_{i} \geq b\right)+P\left(\bar{b}_{i} \leq a\right) \cdot P\left(\bar{a}_{i} \geq b\right) \\
& =2 \cdot F^{s}(a) \cdot\left[1-F^{s}(b)\right]
\end{aligned}
$$

This result allows us to concretize Equation 4.45:

$$
\begin{aligned}
P_{C B R}^{\neg \operatorname{cov}}(a, b, k) & =P(\neg \operatorname{covers}(F \text { List }, F)) \\
& =\left(1-2 \cdot F^{s}(a) \cdot\left[1-F^{s}(b)\right]\right)^{k}
\end{aligned}
$$

This result is closely related to the matching formula $P$ (matches $(F$ List, $n)$ ) given in the previous section. If we set $a=b=x$ and invert the result, we have the same formula $P_{C B R}^{\operatorname{cov}}(x, x, k)=1-P_{C B R}^{\text {match }}(x, k)$. This is because matching is a special case of covering zero length intervals. Equation 4.47 is only correct for $a \leq b$. For $a>b$, we have $P_{C B R}^{\operatorname{cov}}(a, b<a, k)=0$.

Since filter $F$ is also randomly chosen, $P(\neg \operatorname{cover} s(F$ List, $\bar{F}))$ is itself a random variable. The expected probability for not covering a subscription (which equals the subscription forwarding probability) whose limits are distributed according to $f^{s}(\cdot)$ when $k$ subscriptions are active is:

$$
\begin{aligned}
p^{\neg \operatorname{cov}}(k) & =E\left[P_{C B R}^{\operatorname{cov}}(a, b, k)\right] \\
& =\int_{-\infty}^{\infty} \int_{-\infty}^{\infty} f^{s}(a, b) \cdot P^{\neg \operatorname{cov}}(a, b, k) \mathrm{d} b \mathrm{~d} a \\
p_{C B R}^{s}(k) & =\int_{a=-\infty}^{\infty} f^{s}(a) \int_{b=a}^{\infty} f^{s}(b) \cdot\left(1-2 \cdot F^{s}(a) \cdot\left[1-F^{s}(b)\right]\right)^{k} \mathrm{~d} b \mathrm{~d} a
\end{aligned}
$$

The latter integration already considers the fact that $P_{C B R}^{\operatorname{cov}}(a, b, k)$ only exists for $a \leq b$ and that the interval limits are independently selected. In case of a uniform distribution of the interval limits between 0 and 1 for both filter interval limits, i.e., for $f^{s}(\cdot)=1$, we get a simplified formula:

$$
p_{C B R}^{s}(k)=\int_{a=0}^{1} \int_{b=a}^{1}(1-2 \cdot a \cdot[1-b])^{k} \mathrm{~d} b \mathrm{~d} a
$$

With the help of $p_{C B R}^{s}(k)$, we are now able to calculate the expected subscription forwarding probability by substituting $p_{A L}^{s}(k)$ by $p_{C B R}^{s}(k)$ in Equation 4.41 . Unfortunately, a simplification is not possible. Therefore, we get the following:

$$
P_{C B R}^{s}\left(B_{i} B_{j}\right)=\sum_{k=0}^{\infty} q_{k}^{s}\left(B_{i} B_{j}\right) \cdot p_{C B R}^{s}(k)
$$

Merging-based Routing. In merging-based routing a newly issued or canceled subscription with a single interval filter $[a, b]$ at broker $B_{j}$ causes a control message to be propagated to broker $B_{i}$ if all limits $a_{l}$ 's and $b_{l}$ 's of filters $F_{l}$ in $\mathcal{T}\left(B_{i} B_{j}\right)$ are greater than $a$ or if they are all smaller than $b$. Please note that these filter $F_{l}$ do not exist as routing table entries at broker $B_{j}$. Instead, there is one filter that is the merger of these filters. Due to this reason, the calculation of probability of not covering $[a, b]$ with the merger cannot be built 
on the definitions provided in Section 4.4.2. Instead, we directly derive formula from the latter explanation:

$$
P_{M B R}^{\neg c o v}(a, b, k)=\left[1-F^{s}(\bar{a})\right]^{2 k}+\left[F^{s}(\bar{b})\right]^{2 k}-\left[F^{s}(\bar{b})-F^{s}(\bar{a})\right]^{2 k}
$$

The last term is to avoid double counting of cases if all $2 k$ interval limits are covered by the interval $[a, b]$. Again for $b<a$ follows $P_{M B R}^{-c o v}(a, b, k)=0$. Obviously, it is $P_{C B R}^{-c o v}(a, b, 1)=P_{M B R}^{\neg c o v}(a, b, 1)$ which can be tested by showing that $1-2 F^{s}(a)\left[1-F^{s}(b)\right]=\left[1-F^{s}(a)\right]^{2}+\left[F^{s}(b)\right]^{2}-\left[F^{s}(b)-F^{s}(a)\right]^{2}$. The expected value for the probability for not covering which also considers that $a$ and $b$ are randomly chosen according to $f(\cdot)$ can be determined similarly to covering-based routing. Thus, we get the forwarding probability for subscriptions:

$$
\begin{aligned}
p_{M B R}^{s}(k)= & E\left[P_{M B R}^{\neg c o v}(a, b, k)\right] \\
= & \int_{a=-\infty}^{\infty} f^{s}(a) \int_{b=-\infty}^{\infty} f^{s}(b) . \\
& \quad\left(\left[1-F^{s}(\bar{a})\right]^{2 k}+\left[F^{s}(\bar{b})\right]^{2 k}-\left[F^{s}(\bar{b})-F^{s}(\bar{a})\right]^{2 k}\right) \mathrm{d} b \mathrm{~d} a
\end{aligned}
$$

In the special case of uniformly distributed interval limits between 0 and 1 we get:

$$
\begin{aligned}
p_{M B R}^{s}(k) & =2 \cdot \int_{a=0}^{1} \int_{b=a}^{1}(1-a)^{2 k}+b^{2 k}-(b-a)^{2 k} \mathrm{~d} b \mathrm{~d} a \\
& =\frac{1+4 k}{1+3 k+2 k^{2}}
\end{aligned}
$$

If only one subscription is active, there is no possibility to merge anything. Therefore, the forwarding probability of MBR and CBR must be equal. In fact, $p_{C B R}^{s}(1)$ (Equation 4.49) and $p_{M B R}^{s}(1)$ do both equal $\frac{5}{6}$. In Figure 4.14 we want to point out the difference between the continuous formula $\frac{1+4 k}{1+3 k+2 k^{2}}$ and the measured values from a simulation. While the formula returns probabilities for each real value of $k$ including negative and non-integer values, in an experiment we can only measure positive integer values. These inter-integer values have no concrete meaning. Nevertheless, the plot shows that the measured data are exactly located on the plot of the formula.

As a final step for the forwarding probability of subscriptions, we calculate the expected subscription forwarding probability according to Equation 4.41:

$$
P_{M B R}^{s}\left(B_{i} B_{j}\right)=\sum_{k=0}^{\infty} q_{k}^{s}\left(B_{i} B_{j}\right) \cdot \frac{1+4 k}{1+3 k+2 k^{2}}
$$

For comparison, in Figure 4.15, we plotted the discrete forwarding probability $p^{s}$ as well as the expected forwarded probabilities $P^{s}$ for all mentioned routing algorithms. Obviously, the probabilities $P^{s}$ resp. $p^{s}$ can be ordered in the following way: $0=p_{F L}^{s} \leq p_{I B R}^{s} \leq p_{M B R}^{s} \leq p_{C B R}^{s} \leq p_{S R}^{s}=1$ which is the inverse order of the notification forwarding probabilities. Despite flooding, all other routing algorithm start at $100 \%$ for no subscription and monotonically converge to $0 \%$. 


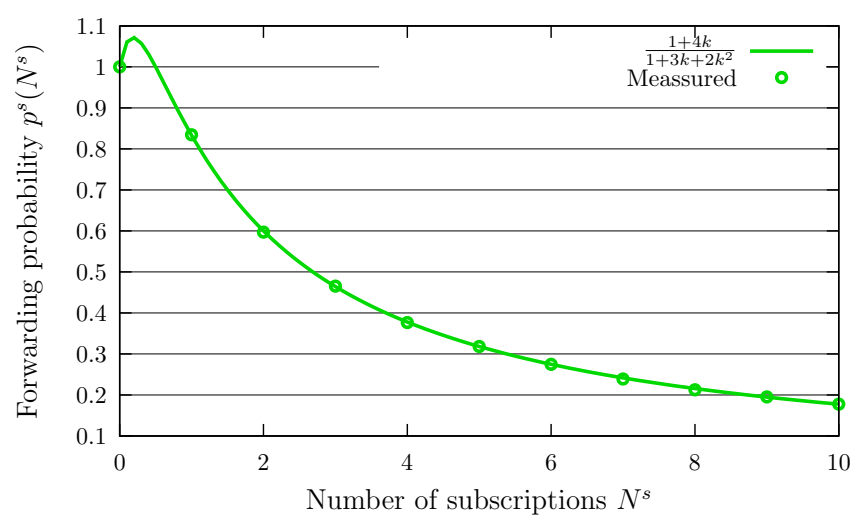

Figure 4.14: Subscription forwarding probabilities for merging-based routing

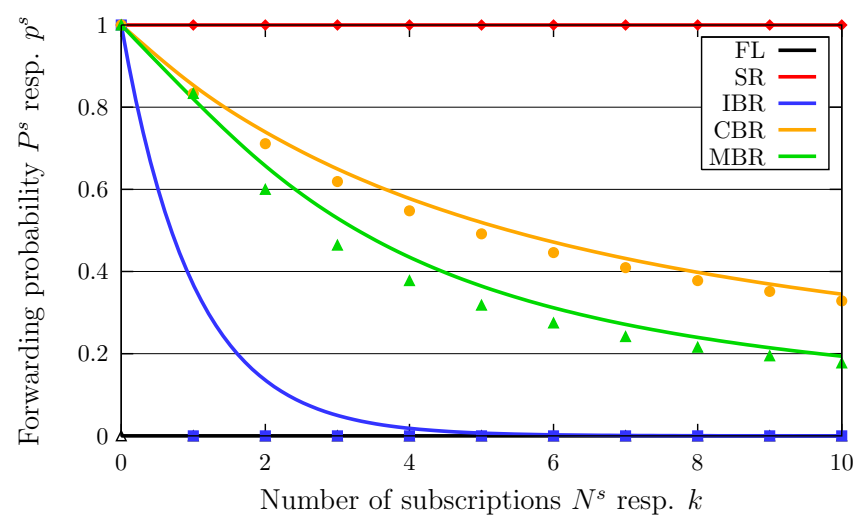

Figure 4.15: Subscription forwarding probabilities 
Advertisement-based Routing. If advertisements are used for routing within a publish/subscribe system, the subscription forwarding probabilities change because a subscription delivery does not only depend on other subscriptions but also on the existence of overlapping active advertisements on the related broker. In case of identity-based routing, a subscription is forwarded if there is no subscription already active and there is at least one active advertisement. We get $p_{I B R}^{s}\left(k^{s}=0, k^{a} \geq 1\right)=1$ otherwise $p_{I B R}^{s}\left(k^{s}, k^{a}\right)=0$. The expected forwarding probability can be calculated as follows:

$$
\begin{aligned}
P_{A+I B R}^{s}\left(B_{i} B_{j}\right) & =E\left[p_{I B R}^{s}\left(k^{s}, k^{a}\right)\right] \\
& =\sum_{k^{a}=0}^{\infty} \sum_{k^{s}=0}^{\infty} q_{k^{a}}^{a}\left(B_{i} B_{j}\right) \cdot q_{k^{s}}^{s}\left(B_{i} B_{j}\right) \cdot p_{I B R}^{s}\left(k^{s}, k^{a}\right) \\
& =\sum_{k^{a}=1}^{\infty} \frac{N^{a}\left(B_{i} B_{j}\right)^{a}}{k^{a} !} \cdot e^{-N^{a}\left(B_{i} B_{j}\right)} \cdot \sum_{k^{s}=0}^{0} \frac{N^{s}\left(B_{i} B_{j}\right)^{k^{s}}}{k^{s} !} \cdot e^{-N^{s}\left(B_{i} B_{j}\right)} \\
& =\left[1-e^{-N^{a}\left(B_{i} B_{j}\right)}\right] \cdot e^{-N^{s}\left(B_{i} B_{j}\right)}
\end{aligned}
$$

This means that the calculation of the expected value of the forwarding probability for Poisson distributed subscriptions and advertisements is the product of the expected forwarding probability of advertisements and the expected forwarding probability of subscriptions without advertisements. This value is always lower than the forwarding probability for simple routing without advertisements.

For content-based routing where the attribute (filter) values are considered, the overlapping probability of a subscription with $k^{a}$ active advertisements has to be determined. According to Equation 4.13 a filter list F List overlaps a filter $J$ if there is at least one filters of the list that overlaps $J$ :

$$
\operatorname{overlaps}(F L i s t, J) \Leftrightarrow \neg \forall i: \operatorname{disjunct}\left(I_{i}, J\right)
$$

When $P(\operatorname{disjunct}(\bar{I}, J))$ is the probability that a randomly chosen filter $\bar{I}$ is disjunct to another filter $J$ with an arbitrary but fixed interval filter $a$ and $b$, we can derive the formula for the probability that an arbitrary filter does not overlap a randomly chosen filter: $\left[F^{s}(a)\right]^{2}+\left[1-F^{s}(b)\right]^{2}$. According to the latter overlap definition for a filter list with $k$ intervals, we multiply the probability and invert the result, getting the following:

$$
\begin{aligned}
P^{\text {olap }}(a, b, k) & =P(\text { overlaps }(F \text { List }, J)) \\
& =1-\left(\left[F^{s}(a)\right]^{2}+\left[1-F^{s}(b)\right]^{2}\right)^{k}
\end{aligned}
$$

This is a generalization of the inverse of the matching relation. For $a=b=$ $x$ we have: $P^{\text {olap }}(x, x, k)=1-P^{\text {match }}(x, k)$. Since advertisement routing is always combined with one of the introduced notification routing algorithms (AL) despite flooding, we have to bring these results together by just multiplying the core probabilities: $p_{A+A L}^{s}\left(k^{s}, k^{a}\right)=E\left[P^{\text {olap }}\left(a, b, k^{a}\right) \cdot P_{A L}^{- \text {cov }}\left(a, b, k^{s}\right)\right]$. Thus, we get the subscription forwarding probability if there are $k^{s}$ active subscription and $k^{a}$ active advertisements: 


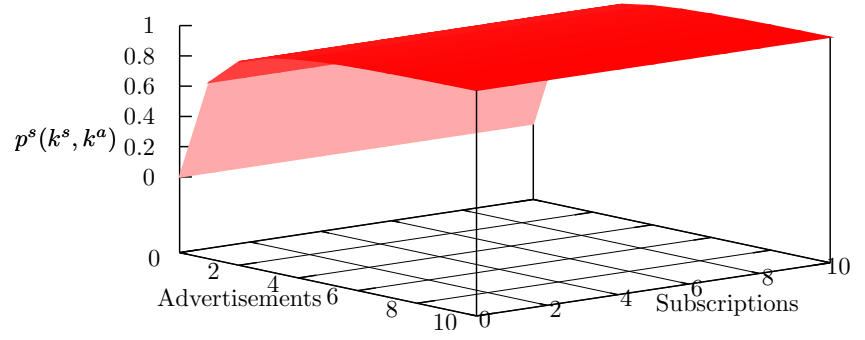

Figure 4.16: Advertisements + SR

$$
\begin{gathered}
p_{A+S R}^{s}\left(k^{s}, k^{a}\right)=E\left[1-\left(\left[F^{s}(a)\right]^{2}+\left[1-F^{s}(b)\right]^{2}\right)^{k^{a}}\right] \\
p_{A+C B R}^{s}\left(k^{s}, k^{a}\right)=E\left[\left(1-\left(\left[F^{s}(a)\right]^{2}+\left[1-F^{s}(b)\right]^{2}\right)^{k^{a}}\right)\right. \\
\left.\left(1-2 F^{s}(a)\left[1-F^{s}(b)\right]\right)^{k^{s}}\right] \\
p_{A+M B R}^{s}\left(k^{s}, k^{a}\right)=E\left[\left(1-\left(\left[F^{s}(a)\right]^{2}+\left[1-F^{s}(b)\right]^{2}\right)^{k^{a}}\right) \cdot\right. \\
\left.\left(\left[1-F^{s}(a)\right]^{2 k^{s}}+\left[F^{s}(b)\right]^{2 k^{s}}-\left(F^{s}(b)-F^{s}(a)\right)^{2 k^{s}}\right)\right]
\end{gathered}
$$

In the special case of uniformly distributed interval limits between 0 and 1 we get:

$$
\begin{aligned}
& p_{A+S R}^{s}\left(k^{s}, k^{a}\right)=2 \cdot \int_{a=0}^{1} \int_{b=a}^{1}\left(1-\left(a^{2}+[1-b]^{2}\right)^{k^{a}}\right) \mathrm{d} b \mathrm{~d} a \\
& p_{A+C B R}^{s}\left(k^{s}, k^{a}\right)=2 \cdot \int_{a=0}^{1} \int_{b=a}^{1}\left(1-\left(a^{2}+[1-b]^{2}\right)^{k^{a}}\right)(1-2 a(1-b))^{k^{s}} \mathrm{~d} b \mathrm{~d} a \\
& p_{A+M B R}^{s}\left(k^{s}, k^{a}\right)=2 \cdot \int_{a=0}^{1} \int_{b=a}^{1}\left(1-\left(a^{2}+[1-b]^{2}\right)^{k^{a}}\right) \cdot \\
& \left((1-a)^{2 k^{s}}+b^{2 k^{s}}-(b-a)^{2 k^{s}}\right) \mathrm{d} b \mathrm{~d} a
\end{aligned}
$$

In Figure 4.16 the influence of the advertisements on the subscription forwarding probability for simple routing is depicted. Clearly, the forwarding probability of subscriptions does only depend on the number of active advertisements since $p_{S R}^{s}\left(k^{s}\right)=1$ for all $k^{s}$. Thus, the graph shows the overlapping probability which starts at 0 and monotonically increases to one with an increasing number of advertisements $k^{a}$. For CBR and MBR with advertisements depicted in Figures 4.17 and 4.18, the overlapping curves are superimposed by the coveringprobabilities of the considered algorithms. Thus, in $k^{s}$ dimension the forwarding probability $p^{s}\left(k^{s}, k^{a}\right)$ decreases while in $k^{a}$ dimension it is increasing. For MBR, we can observe a steeper decrease with increased number of subscriptions. The 


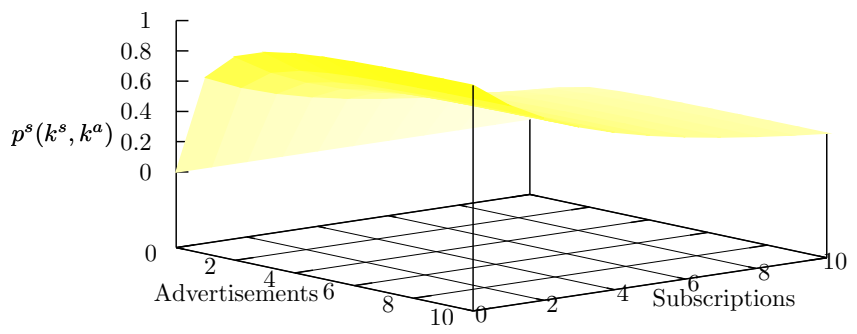

Figure 4.17: Advertisements + CBR

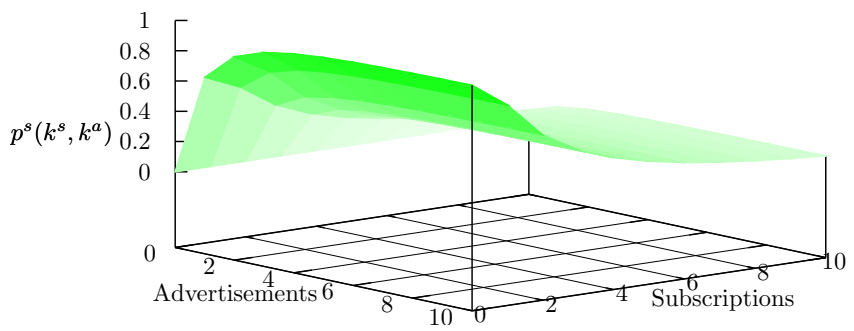

Figure 4.18: Advertisements + MBR

limit of the forwarding probability for $k^{a} \rightarrow \infty$ is the forwarding probability as if no advertisements were used. As expected for all algorithms, the forwarding probability drops most if there are only a few advertisements.

These plots show the forwarding probability for an arbitrary but fixed number of subscriptions and advertisements. If the subscribe and advertise process follows the client model assumption, we can determine the expected forwarding probability for subscriptions with the help of the subscription and advertisement distribution, i.e., $q_{k^{a}}^{a}\left(B_{i} B_{j}\right)$ resp. $q_{k^{s}}^{s}\left(B_{i} B_{j}\right)$. As before, AL represents one of the introduced algorithms:

$$
P_{A+A L}^{s}\left(B_{i} B_{j}\right)=\sum_{k^{a}=0}^{\infty} q_{k^{a}}^{a}\left(B_{i} B_{j}\right) \cdot \sum_{k^{s}=0}^{\infty} q_{k^{s}}^{s}\left(B_{i} B_{j}\right) \cdot p_{A+A L}^{s}\left(k^{s}, k^{a}\right)
$$

This approach decreases the forwarding probability of subscriptions compared to not using advertisements. On the other hand, additional traffic for advertisements as well as a subscription routing table is needed.

\section{Forwarding Probability for Advertisements}

Advertisements which contain filters can also be routed like subscriptions (cf. Section 4.4.7). Thus, it is possible to applied simple routing, identity-based routing, covering-based routing or merging-based routing. Therefore, analogous formulas can be derived which only differ in the used index $a$ instead of $s$, e.g., $f^{s}(\cdot)$ becomes $f^{a}(\cdot)$ etc. This way, the forwarding probability $p_{k}^{a}\left(B_{i} B_{j}\right)$ and finally, the expected value of it $P^{a}\left(B_{i} B_{j}\right)$ can be calculated: 


\begin{tabular}{|c||c|c|c|}
\hline Measure & $\mathrm{AL}$ & $o p$ & $\mathrm{ABR}+\mathrm{AL}$ \\
\hline \hline$P^{n}$ & $P_{A L}^{n}$ & $=$ & $P_{A L}^{n}$ \\
\hline$P^{s}$ & $P_{A L}^{s}$ & $\geq$ & $P_{A+A L}^{s}$ \\
\hline$P^{a}$ & 0 & $\leq$ & $P_{A}^{a}$ \\
\hline$X^{s}$ & $X_{A L}^{s}$ & $\geq$ & $X_{A+A L}^{s}$ \\
\hline$X^{a}$ & 0 & $\leq$ & $X_{A}^{a}$ \\
\hline
\end{tabular}

Table 4.2: Comparison of routing algorithm with/without advertisements

$$
P_{A L}^{a}\left(B_{i} B_{j}\right)=\sum_{k=0}^{\infty} q_{k}^{a}\left(B_{i} B_{j}\right) \cdot p_{A L}^{a}(k)
$$

Similar to subscription forwarding, probabilities $p^{a}$ and $P^{a}$ are monotonic in $N^{a}$ resp. $k$. In Table 4.2, we compare the usage of advertisement-based routing and a standard routing algorithm $(\mathrm{AL})$ without advertisements. The difference between the algorithms is only in terms of control messages. The advantage of AL only is to avoid additional messages and routing tables for advertisements. Advertisement-based routing is in favor since the subscription forwarding probability as well as the notification routing table tend to be smaller. The concrete scenario decides whether the usage of advertisement-based routing is beneficial or not.

\subsubsection{Traffic}

The forwarding probabilities lay the foundation for the calculation of another important performance measure, the traffic. Its unit is messages per time unit. Following we derive different expected traffic measures. We distinguish between incoming and outgoing traffic and we consider different entities, namely brokers, overlay links, underlay links and global measures. We also distinguish between the different types of messages, namely notifications, subscriptions and advertisements. To avoid a multiple repetition of almost equal formulas, we use $x$ as a placeholder for the message types: $n, s$ and $a$. Thus, $P^{x}\left(B_{i} B_{j}\right)$ is the probability that a message of type $x$ is propagated from broker $B_{i}$ to a neighbor broker $B_{j}$. Similar to Section 4.4.5, we first introduce an auxiliary measure. If a message of type $x$ is issued at broker $B_{i}$, it is propagated into the subtopology $\mathcal{T}\left(B_{i} B_{j}\right)$ causing an (expected) number of forwardings:

$$
P_{a c c}^{x}\left(B_{i} B_{j}\right)=P^{x}\left(B_{i} B_{j}\right)+\sum_{N \in \mathcal{S}\left(B_{i} B_{j}\right)} P_{a c c}^{x}(B N)
$$

Overlay Traffic. We start with incoming traffic. Let $\nu_{i n}^{x}\left(B_{i} B_{j}\right)$ be the overall incoming traffic of type $x$ that potentially reaches $B_{i} B_{j}$ (if not filtered). It is used to define the effective forwarding probability (cf. Section 4.4.7). This measure is the sum of the local generated traffic and overlay link traffic of all predecessing brokers. Thus, we get:

$$
\nu_{\text {in }}^{x}\left(B_{i} B_{j}\right)=\lambda^{x}\left(B_{i}\right)+\sum_{N \in \mathcal{P}\left(B_{i} B_{j}\right)} \nu_{\text {out }}^{x}\left(N B_{i}\right)
$$


The incoming traffic of type $x$ on a broker $B$ consists of the traffic received by its local clients and those received from its neighbor brokers. It can be used to identify brokers with high utilization or bottlenecks. According to the verbal definition $\nu_{i n}^{x}(B)$ is formalized as follows:

$$
\nu_{\text {in }}^{x}(B)=\lambda^{x}(B)+\sum_{N \in \mathcal{N}(B)} \nu_{\text {out }}^{x}(N B)
$$

Please note that in the latter equation $\lambda^{x}(B)$ is a general birth rate which is in case of notifications the publication rate $\omega(B)$. Later in this section, we provide a table with necessary implications for all message types.

After the incoming traffic we come to outgoing traffic. The message traffic $\nu_{\text {out }}^{x}\left(B_{i} B_{j}\right)$ is the number of messages of type $x$ per time unit that broker $B_{i}$ forwards to $B_{j}$. This measure provides the traffic on the overlay link $\nu_{\text {out }}^{x}\left(B_{i} B_{j}\right)$ and can be further used to determine utilizations, possible bottlenecks and delays of links. It is calculated by the forwarding probability and the accumulated rates (cf. Section 4.4.5):

$$
\nu_{\text {out }}^{x}\left(B_{i} B_{j}\right)=P^{x}\left(B_{i} B_{j}\right) \cdot \lambda_{a c c}^{x}\left(B_{i} B_{j}\right)
$$

Since messages are not generated by brokers, it holds: $\nu_{\text {out }}^{x}\left(B_{i} B_{j}\right) \leq \nu_{i n}^{x}\left(B_{i} B_{j}\right)$. In case that $P^{x}\left(B_{i} B_{j}\right)=1$, it results that $\nu_{\text {out }}^{x}\left(B_{i} B_{j}\right)=\lambda_{\text {acc }}^{x}\left(B_{i} B_{j}\right)$ which is the case for notifications in flooding and subscriptions in simple routing. This means that the traffic on overlay link $B_{i} B_{j}$ is equal to the accumulated birth rate in the subtopology $\mathcal{T}\left(B_{j} B_{i}\right)$.

The outgoing traffic $\nu_{\text {out }}^{x}(B)$ of type $x$ generated by a broker $B$ can be used to determine which fraction of the traffic in the entire network is generated by this broker. $\nu_{\text {out }}^{x}(B)$ is calculated by the product of the local birth rate of messages with accumulated probability $P_{a c c}^{x}(B N)$ :

$$
\nu_{\text {out }}^{x}(B)=\lambda^{x}(B) \cdot \sum_{N \in \mathcal{N}(B)} P_{a c c}^{x}(B N)
$$

After having derived formulas for incoming and outgoing message traffics on brokers and overlay links, we finally derive the global performance measure $\nu^{x}$ which is the total traffic of messages of type $x$ in the network:

$$
\nu^{x}=\sum_{B \in \mathcal{B}} \nu_{\text {out }}^{x}(B)=\sum_{B \in \mathcal{B}} \nu_{\text {in }}^{x}(B)=\sum_{B_{i} B_{j} \in \mathcal{E}} \nu_{\text {out }}^{x}\left(B_{i} B_{j}\right)
$$

Figures 4.19 visualize the traffic measures for a broker $B_{i}$ resp. for an overlay link $B_{i} B_{j}$. By the color of the arrows we marked those parts of the traffic that the incoming and outgoing traffic are consisting of. As already described, the incoming traffic for brokers and links is the sum of some other message flows of the related type. The outgoing traffic of a link is simply the traffic on this link, but for a broker it is the traffic in the whole network generated by the subsequent delivery of messages locally produced at the broker.

As already mentioned, all measures have a superscript $x$ for an arbitrary message type. Depending on the interested message type they have to be instantiated as shown in Table 4.3. The factor 2 for the birth rates of subscriptions and advertisements stems from the fact that each of these messages has its counterpart, the unsubscription resp. the unadvertisement. In case of subscription 


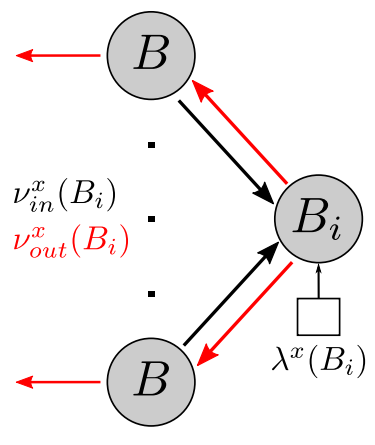

(a) Broker traffic

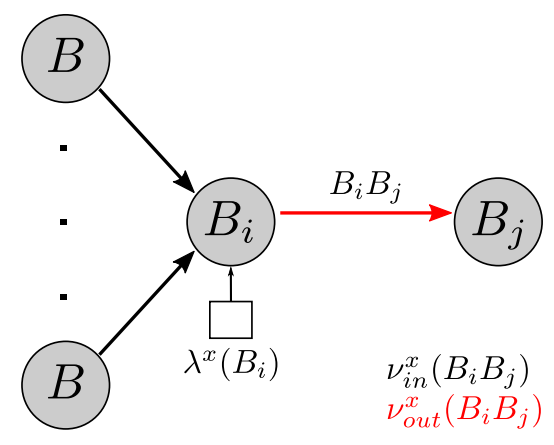

(b) Link traffic

Figure 4.19: Traffic measures

\begin{tabular}{|c||c|c|c|}
\hline Measure/Message & Notification & Subscription & Advertisement \\
\hline \hline$\lambda^{x}(B)$ & $\omega$ & $2 \cdot \lambda^{s}$ & $2 \cdot \lambda^{a}$ \\
\hline$\lambda_{a c c}^{x}\left(B_{i} B_{j}\right)$ & $\omega_{a c c}$ & $2 \cdot \lambda_{a c c}^{s}$ & $2 \cdot \lambda_{a c c}^{a}$ \\
\hline$P_{a c c}^{x}\left(B_{i} B_{j}\right)$ & $P_{a c c}^{n}$ & $P_{a c c}^{s}$ & $P_{a c c}^{a}$ \\
\hline$P^{x}\left(B_{i} B_{j}\right)$ & $P^{n}$ & $P^{s}$ & $P^{a}$ \\
\hline \hline$\nu_{\text {in }}^{x}\left(B_{i} B_{j}\right)$ & $\nu_{i n}^{n}$ & $\nu_{\text {in }}^{s}(*)$ & $\nu_{\text {in }}^{a}$ \\
\hline$\nu_{\text {in }}^{x}(B)$ & $\nu_{i n}^{n}$ & $\nu_{\text {in }}^{s}$ & $\nu_{\text {in }}^{a}$ \\
\hline$\nu_{\text {out }}^{x}\left(B_{i} B_{j}\right)$ & $\nu_{\text {out }}^{n}$ & $\nu_{\text {out }}^{s}$ & $\nu_{\text {out }}^{a}$ \\
\hline$\nu_{\text {out }}^{x}(B)$ & $\nu_{\text {out }}^{n}$ & $\nu_{\text {out }}^{s}$ & $\nu_{\text {out }}^{a}$ \\
\hline$\nu^{x}$ & $\nu^{n}$ & $\nu^{s}$ & $\nu^{a}$ \\
\hline
\end{tabular}

Table 4.3: Message type dependencies

messages (marked by "*" in the table), we have to invert the calculation of the flow leading to $\nu_{i n}^{s}\left(B_{i} B_{j}\right)=\lambda_{a c c}^{s}\left(B_{j}\right)+\sum_{N \in \mathcal{S}\left(B_{i} B_{j}\right)} \nu_{\text {out }}^{x}\left(B_{j} N\right)$. This is due to the inverted flow of subscriptions.

A special type traffic which we did not add to the Table 4.3 is the one of false positive notifications which is a direct outcome of the discussion about forwarding probabilities of notifications in Section 4.4.7. We can easily define the overlay link traffic of FP notifications similarly to the one of $\nu_{\text {out }}^{n}\left(B_{i} B_{j}\right)$ :

$$
\nu_{\text {out }}^{\bar{n}}\left(B_{i} B_{j}\right)=P^{\bar{n}}\left(B_{i} B_{j}\right) \cdot \omega_{a c c}\left(B_{i} B_{j}\right)
$$

Total Traffic. After the derivation of measures that are related to all considered message types we are now able to bring them together into one measure by just adding all message flows: The total traffic ${ }^{3}$ on overlay link $B_{i} B_{j}$, the total traffic received by broker $B$, the total traffic generated by $B$ and the total traffic in the entire network are then:

\footnotetext{
${ }^{3}$ We use global traffic and overall traffic as synonyms.
} 


$$
\begin{aligned}
\nu_{\text {out }}\left(B_{i} B_{j}\right) & =\nu_{\text {out }}^{n}\left(B_{i} B_{j}\right)+\nu_{\text {out }}^{s}\left(B_{i} B_{j}\right)+\nu_{\text {out }}^{a}\left(B_{i} B_{j}\right) \\
\nu_{\text {in }}(B) & =\nu_{\text {in }}^{n}(B)+\nu_{\text {in }}^{s}(B)+\nu_{\text {in }}^{a}(B) \\
\nu_{\text {out }}(B) & =\nu_{\text {out }}^{n}(B)+\nu_{\text {out }}^{s}(B)+\nu_{\text {out }}^{a}(B) \\
\nu & =\nu^{n}+\nu^{s}+\nu^{a}
\end{aligned}
$$

These measures are important since they can directly be mapped to costs for the necessary bandwidth of links and to the required broker configuration.

Underlay Traffic. An overlay link $B_{i} B_{j}$ connects two brokers in the overlay topology by a path in the underlying physical network consisting of physical links and routers. Since "in general, Internet paths are strongly dominated by a single route." [78] this assumption is a good approximation even for publish/ subscribe layered on top of the Internet. On the other hand, each physical component can be part of several overlay links. The traffic generated by an arbitrary message type $x$ on a physical component $\bar{l}$ can be written as:

$$
\nu_{\text {out }}^{x}(\bar{l})=\sum_{\bar{l} \in\left(B_{i} B_{j}\right)} \nu_{\text {out }}^{x}\left(B_{i} B_{j}\right)
$$

With $\bar{l} \in\left(B_{i} B_{j}\right)$, we mean the set of all overlay links that contain $\bar{l}$. For a total traffic measure of one physical component we can again sum up all message types:

$$
\nu_{\text {out }}(\bar{l})=\nu_{\text {out }}^{n}(\bar{l})+\nu_{\text {out }}^{s}(\bar{l})+\nu_{\text {out }}^{a}(\bar{l})
$$

The latter measure is important to determine the load on underlay links and to detect possible bottlenecks.

\subsubsection{Utilization and Delay}

The utilization $U$ is the proportion of the system's capacity $C$ which is used by the traffic $T$ arriving at the system. Thus, the definition is $U=T / C$ which is a unit less measure. For a stable system, the utilization must be strictly less than one. If this is not the case, the system will be overloaded and will not function anymore. Depending on the system behavior often modeled as a queueing system the utilization directly determines the delay of messages. Following, we derive formulas for the utilization and delay for brokers and links.

Broker Utilization and Delay. Each broker is able to handle a certain amount of incoming messages per seconds. Thus, the utilization of the broker does only depend on the traffic of the incoming messages and its capacity. Thus, we assume that the broker is network bound independently from the message type. Using the latter definition, the overall utilization of broker $B$ caused by the notifications and control messages is:

$$
U(B)=\frac{\nu_{\text {in }}(B)}{c(B)}
$$


where $c(B)$ is the broker capacity. Since we model brokers as $M / M / 1$ queues, we can determine the expected broker delay $D(B)$ of a message at broker $B$ with the well-known formula [52]:

$$
D(B)=\frac{1}{c(B) \cdot[1-U(B)]}
$$

where $1 / c(B)$ is the mean processing time of a message at broker $B$. Obviously, if the utilization approaches $100 \%$ which is an overload situation, the delay $D(B)$ tends to $\infty$. An infinite delay includes the $M / M / 1$ assumption to have an infinite message queue. In real systems, this is never the case and from a certain point of time incoming messages have to be dropped. Nevertheless, we use the $M / M / 1$ model that is simpler than a $M / G / 1$ system and often a sufficient approximation [90].

Utilization and Delay of Physical Components. The utilization $U(\bar{l})$ of a component $\bar{l}$ can be determined similar to Equation 4.78:

$$
U(\bar{l})=\frac{\nu_{\text {out }}(\bar{l})}{c(\bar{l})}
$$

Note that if $U(\bar{l})>1$ for any physical component, the system is overloaded and not in a steady state. The expected delay of a physical component is again similar to Equation 4.79 since we again have a $M / M / 1$ behavior:

$$
D(\bar{l})=\frac{1}{c(\bar{l}) \cdot[1-U(\bar{l})]}
$$

As for the related traffics, these utilizations are important to determine bottlenecks in the publish/subscribe infrastructure. An operator or designer should always check whether the utilizations of all involved components are below a predefined threshold including a safety margin.

Delay of Overlay Links. After the delay of the physical component we are now able to derive the delay of a logical overlay link $D\left(B_{i} B_{j}\right)$. Since both directions of an overlay link use the same physical components, the overlay link delay is symmetric, thus $D\left(B_{i} B_{j}\right)=D\left(B_{j} B_{i}\right)$. If the physical components do not have this symmetry the model can be easily extended by redefining Equation 4.76. In this thesis, we determine the delay of an overlay link by adding up the delays at its constituting physical components:

$$
D\left(B_{i} B_{j}\right)=\sum_{\bar{l} \in B_{i} B_{j}} D(\bar{l})
$$

The calculated delays for brokers and overlay links are the foundation to calculate the maximum expected notification delay and the maximum expected subscription delay which are both important performance measures for clients.

Notification Delay. As discussed in Section 4.3.1, the notification delay is the time it takes before an issued notification reaches the last subscriber. To derive a global measure, we first define the maximum expected delay of an overlay link $B_{i} B_{j}$. It is calculated recursively as follows: 


$$
D^{n}\left(B_{i} B_{j}\right)=P^{n}\left(B_{i} B_{j}\right) \cdot\left[D\left(B_{i} B_{j}\right)+D\left(B_{j}\right)\right]+\max _{N \in \mathcal{S}\left(B_{i} B_{j}\right)} D^{n}\left(B_{j} N\right)
$$

This recursive definition uses two basic properties of the max operator, namely $\max (a+c, b+c)=c+\max (a, b)$ and $\max (a, b, c)=\max (\max (a, b), c)$, to determine the path with the maximum expected delay. Since notifications are issued at brokers we finally add the processing time of the broker and determine the broker with the maximum expected delay:

$$
\Delta^{n}=\max _{B \in \mathcal{B}}\left[D(B)+\max _{N \in \mathcal{N}(B)} D^{n}(B N)\right]
$$

Subscription Delay. Similar to the notification delay, the delay of a subscription issued by a client of a broker $B$ is calculated recursively based on a link related auxiliary measure:

$$
D^{s}\left(B_{i} B_{j}\right)=P^{s}\left(B_{i} B_{j}\right) \cdot\left[D\left(B_{i} B_{j}\right)+D\left(B_{i}\right)\right]+\max _{N \in \mathcal{P}\left(B_{i} B_{j}\right)} D^{s}\left(N B_{i}\right)
$$

It is important to note that in the latter formula the direction has been inverted due to the inverse flow of subscriptions compared to notifications. To derive a global measure, we determine the broker $B$ with the largest delay as follows:

$$
\Delta^{s}=\max _{B \in \mathcal{B}}\left[D(B)+\max _{N \in \mathcal{N}(B)} D^{s}(B N)\right]
$$

This is the final step of the derivation of the stochastic system model of a distributed content-based publish/subscribe system. Finally, it consists of around 80 formulas. Despite some restrictions which we overcome in Section 4.6 the model is complete. It allows to determine all important performance and cost measures required by clients and operators of a publish/subscribe system. The model uses all necessary input parameters, namely the configuration and the environment. The applicability of the model is demonstrated in next section. There, we show how it can be used to get interesting insights which sometimes match with intuitive and qualitative results given in literature, but sometimes not. Furthermore, in the separate Chapter 5 we provide a strategy for capacity planning of publish/subscribe systems which is based on the presented model. In this chapter, we also describe the challenges in the calculation of the complete model and how we tackled them.

\subsection{Evaluation}

In this evaluation section we first shortly evaluate the stochastic system model by simulations. Then, we provide interesting insights about routing algorithms that we generated by the application of the model. The results are based on the derived formulas and have been checked by a discrete event simulation. Furthermore, we evaluate the model by a case study about hierarchical routing. We have chosen hierarchical routing because it is the most interesting one due to its asymmetric routing. Furthermore, the analysis of hierarchical routing brings us a step closer to a new kind of routing introduced in Chapter 6 . 


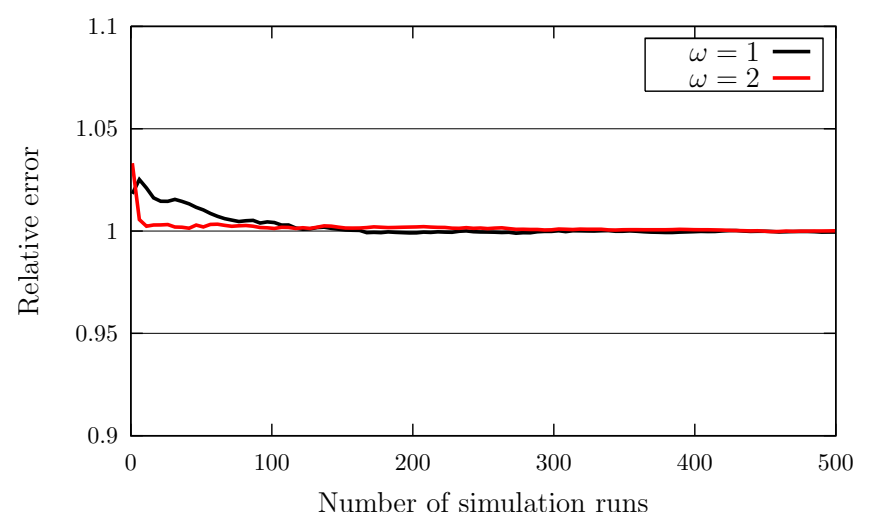

Figure 4.20: Remote routing entries

\subsubsection{Simulation}

To check the validity of the presented formulas, we implemented a discrete event simulation in JAVA. It simulates the behavior of all components, such as brokers, clients, routers, etc. in the same way as we have described it in Section 4.4. Inputs and outputs of the simulation are the same as we explained it in Section 4.3.

The simulation works as follows: when the simulation is started, the system is empty, i.e., it contains no subscribers. Then, the number of subscribers increases until a stable state is reached in that the number of subscribers that are born and that die per time unit are equal on average. After the system has approximately reached the number of intended subscriptions, measurements are taken.

In Figure 4.20 we exemplary show how the simulation result converges to the analytic result of the stochastic model if the number of simulation runs is increased. The simulated overlay consists of four brokers and flooding. The publication rate on one broker is $\omega=1 s^{-1}$ resp. $\omega=2 s^{-1}$. We measure resp. calculate the total notification traffic $\nu^{n}$ according to Equation 4.70. The figure shows that the ratio between the simulation result and the analytic result quickly converges to 1. Obviously, in this experiment the convergence speed depends on the publication rate. This is because of the higher standard deviation of a single simulation run. Therefore, especially for small rates the number of experiments must be sufficiently high to get good results. Beside the depicted result we validated the model for a big variety of inputs and outputs which makes us sure that the model is correctly derived. Furthermore, we compared measurements of our middleware REBECA [73] with the output of the analysis with a positive result. In the following, we use the stochastic model as often as possible because it can be calculated much faster than conducting expensive simulations (cf. Section 5.3.3).

\subsubsection{Insights}

As often mentioned, the selection of the routing algorithm is a crucial point for publish/subscribe systems. To simplify this consideration, in this section we focus on the differences between the algorithms on an overlay link. 


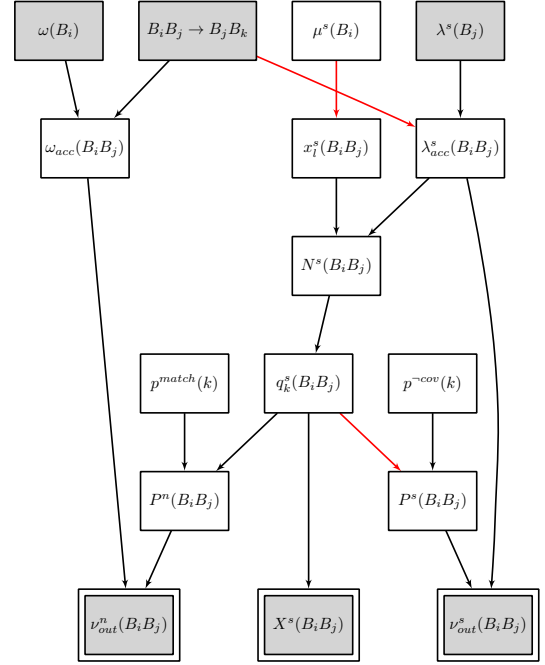

(a) Full dependencies

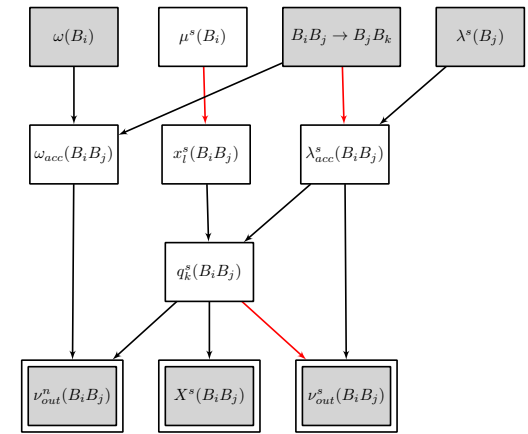

(b) Reduced dependencies

Figure 4.21: Qualitative dependencies of measures

Qualitative Dependencies. We start with Figure 4.21(a) that depicts the qualitative dependencies of all important cost measures, namely traffics and the routing table size, related to an overlay link $B_{i} B_{j}$. Quantities that are connected by an arrow directly depend on each other. The direction of the arrow shows the causal dependency. For example, $\lambda^{s}\left(B_{i} B_{j}\right) \rightarrow \lambda_{a c c}^{s}\left(B_{j}\right)$ symbolizes a function $\lambda_{a c c}^{s}=f\left(\lambda^{s}\right)$ which is described by Equation 4.16. Interestingly, all depicted formulas are monotonic functions either monotonically increasing or monotonically decreasing. We mark this difference in the figure by a black resp. a red colored arrow. Most arrows such as $\lambda^{s} \rightarrow \lambda_{\text {acc }}^{s}$ are black which in this case means that the accumulated subscription arrival rate $\lambda_{a c c}^{s}$ of link $B_{i} B_{j}$ increases if the subscription rate $\lambda^{s}$ of broker $B_{j}$ increases and vice versa. Since a sequence of monotonically increasing functions is again monotonically increasing, we can simplify the dependency graph as depicted in Figure 4.21(b). Furthermore, in this figure we removed the constant inputs $p^{\text {match }}(k)$ and $p^{\neg c o v}(k)$. On the other hand, there are three red arrows in the figures with monotonically decreasing dependencies such as $q_{k}^{s}\left(B_{i} B_{j}\right) \rightarrow P^{s}\left(B_{i} B_{j}\right)$, which means that a higher probability for subscriptions reduces the forwarding probability of it. Beside direct dependencies, the monotony of the functions helps us to determine the dependencies of not directly connected quantities by just following the path from the starting quantity to the destination. If we count an even number of red arrows, the overall dependency is monotonically increasing. Otherwise, it is decreasing. For example, the qualitative dependency of $\lambda^{s}\left(B_{j}\right) \rightarrow P^{s}\left(B_{i} B_{j}\right)$ is a monotonically decreasing function since on the path is only one inverting behavior. If there is more than one path between two considered quantities, such as for $\lambda^{s}\left(B_{j}\right) \rightarrow \nu_{\text {out }}^{s}\left(B_{i} B_{j}\right)$, a statement about the dependency can only be made if each single path results in the same monotony. In the example, this is not the case and we get an indifferent result. Thus, only a detailed analysis of the formulas allows to describe the dependency. 
With this knowledge we can derive an important insight about the change of the performance measures if we move from one overlay link to another. The central box in Figure 4.21(a) is the starting point: $\left[B_{i} B_{j} \rightarrow B_{j} B_{k}\right]$. It symbolizes a shift from $B_{i} B_{j}$ to one of its successing neighbor links $B_{j} B_{k}$. By applying the mentioned monotony rules one can determine the implications on $\nu_{\text {out }}^{n}, \nu_{\text {out }}^{s}$ and $X^{s}$. While the publication rate increases when going down the overlay, the birth rate of subscriptions decreases. This opposing behavior is mainly caused by the inverted traffic definition of notifications and subscriptions. For notifications, the shift $\left[B_{i} B_{j} \rightarrow B_{j} B_{k}\right]$ adds broker $B_{j}$ to the set of brokers that may host publishers. It holds subtopology $\mathcal{T}\left(B_{k} B_{j}\right)=\left\{B_{j}\right\} \cup \mathcal{T}\left(B_{j} B_{i}\right)$. On the other hand, this operation removes broker $B_{j}$ from the set of brokers that potentially host subscribers.

Since there is only one path between $\left[B_{i} B_{j} \rightarrow B_{j} B_{k}\right]$ and $X^{s}$ with one inverting arrow we can directly see that the routing table size will decrease when going down an overlay link. This is a very intuitive behavior because less and less subscribers cause less and less routing entries. For the traffic, the situation is more difficult. In both cases, there are two paths between $\left[B_{i} B_{j} \rightarrow B_{j} B_{k}\right]$ and $\nu_{\text {out }}^{n}$ resp. $\nu_{\text {out }}^{s}$ with contrary statements about monotony. For the notification traffic, the shift of the overlay link increases the accumulated publication rate but the reduction of the accumulated birth rate of subscriptions reduces the forwarding probability of notifications. Both effects may be superimposed in different ways. Thus, a general qualitative statement cannot be given. This indifference can only be solved if $P^{n}$ is pinned. This is the case of flooding with a forwarding probability of 1 . Thus, in flooding the notification traffic always increases when moving downstream. For the subscription traffic $\nu_{\text {out }}^{s}$ a similar discussion is possible. Here, the indifferent result can be solved by pinning $P^{s}$ which is the case for flooding and simple routing.

Routing Algorithms. Following, we consider the influence of increased rates on the cost measures of an overlay link. Figure 4.21(a) already shows that an increasing publication rate $\omega$ on a broker increases the accumulated rate $\omega_{\text {acc }}$ (if it is part of the related subtopology) and this increases the notification traffic $\nu_{o u t}^{n}$ due to the formula $\nu_{\text {out }}^{n}=P^{n} \cdot \omega_{\text {acc }}$. Since this is the only path, the notification traffic on an overlay link for an arbitrary publication rate can be ordered and is determined by the ordering of $P^{n}$ which depends on the routing algorithm (cf. Section 4.4.7). Thus, we get the following order of the notification traffic depending on the routing algorithm: $\omega_{\text {acc }}=\nu_{\text {out }}^{n}(F L) \geq \nu_{\text {out }}^{n}(I B R) \geq$ $\nu_{\text {out }}^{n}(M B R) \geq \nu_{\text {out }}^{n}(C B R)=\nu_{\text {out }}^{n}(S R) \geq 0$. We skip a plot of this linear functions because they do not provide any insights. The influence of $\lambda^{s}$ resp. $N^{s}$ on the number of remote routing entries $X^{s}$ we already plotted in Section 4.4.6.

The influence of $\lambda^{s}$ on the subscription traffic $\nu_{\text {out }}^{s}$ is indifferent due to two possible paths in the Figure 4.21(a). On the one hand, $\lambda^{s}$ increases the subscription traffic $\nu_{\text {out }}^{s}$ via $\lambda_{\text {acc }}^{s}$. The more subscriptions are issued, the more are likely to be delivered. On the other hand, a higher rate $\lambda^{s}$ reduces the forwarding probability of subscriptions $P^{s}$ which is specially true for advanced routing algorithms. From intuition it is not clear which of both contrary effects has a stronger influence on the subscription traffic of the related overlay link. Thus, we plotted the behavior in Figure 4.22 assuming a subscription lifetime of one second. Since $\mu^{s}$ is a factor for $\nu_{\text {out }}^{s}$ in all equations it only scales the results but 


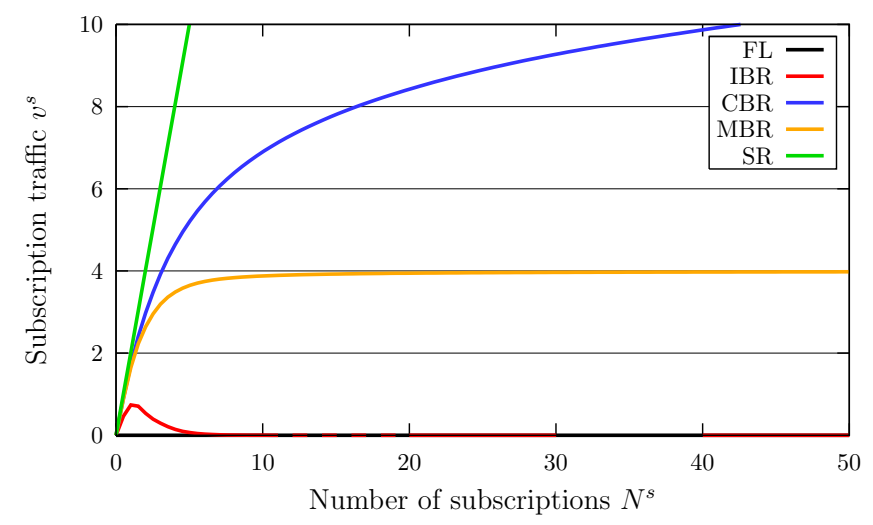

Figure 4.22: Subscription traffic for different routing algorithms

does not change the qualitative behavior. Flooding and simple routing show a very intuitive behavior. While $F L$ does not generate any subscription traffic, traffic of $S R$ grows linearly. The other algorithms show a different behavior. For identity-based routing the maximum flow of notifications is reached at $N^{s}=1$. This can be easily shown by analyzing the formula of the subscription traffic which is $\nu_{\text {out }}^{s}=P_{I B R}^{s} \cdot \lambda_{a c c}^{s}=e^{-N^{s}} \cdot N^{s} / \mu^{s}$. The maximum can be determined by setting $\frac{d \nu_{\text {out }}^{s}}{d N^{s}}=0$. After the maximum, the traffic quickly converges to 0 since it becomes more and more unlikely that there is no active subscription in the considered subtopology. For covering-based and merging-based routing we do not have a maximum in the traffic. Here the subscription traffic grows monotonically. Nevertheless, the increase of the traffic drops the more subscriptions are active in the subtopology. This behavior shows that the forwarding probability of subscriptions decreases stronger than the subscription traffic increases. In case of MBR, the decrease of $P^{s}$ is strong enough to limit the subscription traffic. This limit can be determined starting with Equation 4.41:

$$
\begin{aligned}
\nu_{\text {out }}^{s} & =P_{M B R}^{s} \cdot \lambda_{\text {acc }}^{s} \\
& =N^{s} / \mu^{s} \cdot \sum_{k=0}^{\infty} q_{k}^{s}\left(N^{s}\right) \cdot \frac{1+4 k}{1+3 k+2 k^{2}} \\
& \approx N^{s} / \mu^{s} \cdot \frac{1+4\left(N^{s} / 2\right)}{1+3\left(N^{s} / 2\right)+2\left(N^{s} / 2\right)^{2}}, N^{s}>>1 \\
\lim _{N^{s} \rightarrow \infty} \nu_{\text {out }}^{s} & =4 / \mu^{s}
\end{aligned}
$$

The derivation steps include an approximation for expected value of the Poisson distribution for large $N^{s}$ and the calculation of the limit for $N^{s} \rightarrow \infty$. Interestingly, for covering-based routing $\nu_{\text {out }}^{s}$ is diverging since the probability $P_{C B R}^{s}\left(N^{s}\right)$ is dropping slower than $1 / N^{s}$. Nevertheless, the increase of the subscription traffic seems to be very smooth, similar to $\sqrt{N^{s}}$ or even $\log \left(N^{s}\right)$. Although, the subscription traffic is not monotonically increasing for all algorithms, we are able to order it as following: $0=\nu_{\text {out }}^{s}(F L) \leq \nu_{\text {out }}^{s}(I B R) \leq$ $\nu_{\text {out }}^{s}(M B R) \leq \nu_{\text {out }}^{s}(C B R)=\nu_{\text {out }}^{s}(S R)=2 \cdot \lambda_{\text {acc }}^{s}$. This result equals to the ordering of $P^{s}$ provided in Section 4.4.7. 


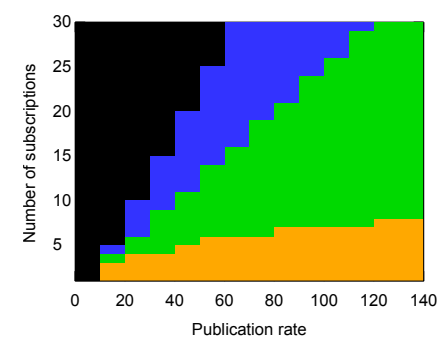

(a) $\mu^{s}=1$

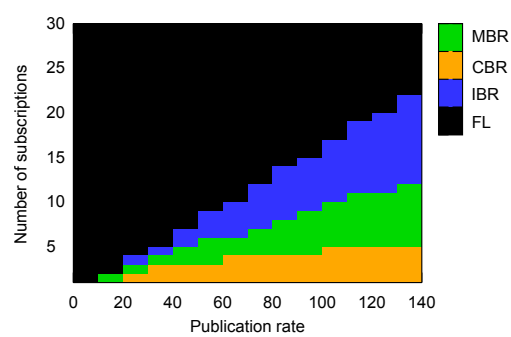

(b) $\mu^{s}=3^{-1}$

Figure 4.23: Comparison between different routing algorithms

So far, we considered notification and subscription traffic on an overlay link separately. In order to determine which of the presented algorithms is the best according to the generated traffic we now just add both the following way: $\nu_{\text {out }}\left(\omega_{\text {acc }}, N^{s}\right)=\nu_{\text {out }}^{n}\left(\omega_{a c c}, N^{s}\right)+\nu_{\text {out }}^{s}\left(N^{s}\right)$. In the latter equation, we added in brackets the quantities the formulas depend on. To get a better idea about the optimal algorithms for different $\omega_{a c c}$ and $N^{s}$, we plotted two Figures 4.23 with a fixed subscription lifetime of 1 resp. 3 seconds. The color represents the optimal algorithm for the related parameter set $\left(\omega_{a c c}, N^{s}\right)$. Obviously with an increasing lifetime (correlating to an increased $\lambda_{a c c}^{s}$ ) flooding overtakes more and more of the parameter space. This is clear due to the fast increasing probability that clients are interested in most of the publications which prefers FL. The other algorithms can be ordered depending on the dimension similar to $\nu_{\text {out }}^{n}$ resp. $\nu_{\text {out }}^{s}$. In the subscription dimension, covering-based routing is preferred for very low number of subscriptions, because it is a perfect routing algorithm and it reduces its control traffic efficiently. For a greater number of subscriptions, merging-based routing takes the lead, because its control traffic is smaller than for covering-based routing and the false positive notifications traffic is already small enough. Then, identity-based routing is the optimal algorithm with a very small control traffic and almost flooding like behavior. Finally, for a large number of subscriptions, clients are interested in almost all notifications which prefers flooding. Simple routing is never the best in both figures. The reason for that is that SR causes the same notification traffic as CBR but generates more control traffic. Thus, SR could only be beneficial to use if the message size of control messages is considered or if other cost measures such as the processing of the covering relation are taken into account.

Although in Figures 4.23 the borders between the algorithms seem to be linear functions, most of them have more complex descriptions that are not easy to explicitly determine. Nevertheless, for the border between FL and IBR it is possible. For the determination, we equalize the traffics of both algorithms, substitute the formulas from Section 4.4.7 and simplify the equation: 


$$
\begin{aligned}
P_{F L}^{n} \cdot \omega_{a c c}+2 \cdot P_{F L}^{s} \cdot \lambda_{a c c}^{s} & =P_{I B R}^{n} \cdot \omega_{a c c}+2 \cdot P_{I B R}^{s} \cdot \lambda_{a c c}^{s} \\
\omega_{a c c} & =\left(1-e^{-\frac{\lambda_{a c c}^{s}}{\mu^{s}}}\right) \cdot \omega_{a c c}+2 \cdot e^{-\frac{\lambda_{c c c}^{s}}{\mu^{s}} \cdot \lambda_{a c c}^{s}} \\
e^{-\frac{\lambda_{a c c}^{s}}{\mu^{s}} \cdot \omega_{a c c}} & =e^{-\frac{\lambda_{a c c}^{s}}{\mu^{s}}} \cdot 2 \cdot \lambda_{a c c}^{s} \\
\omega_{a c c} & =2 \cdot \lambda_{a c c}^{s}=2 \cdot N^{s} / \mu^{s}
\end{aligned}
$$

That last equation provides a linear function that describes the line in both Figures 4.23 with the slopes $1 / 2$ and $1 / 6$. Thus, the slope increases with increasing lifetimes. Actually, at these borders the algorithm should be changed which we eventually do in Section 6 .

A summary of the order of all performance measures $(\mathrm{M})$ related to an overlay link $B_{i} B_{j}$ depending on the selected routing algorithm (AL) is depicted in Table 4.4. For the compactness of the representation we left out the parameter $B_{i} B_{j}$ for many quantities.

\subsubsection{Hierarchical Routing}

In opposite to the other sections, where we had only a local view on publish/ subscribe systems, in this section we present an analysis of an entire system. As an addon, we analyze hierarchical routing instead of peer-to-peer routing which we did not consider in the analysis but which is often suggested to be a good alternative to other routing schemes. In order to derive the performance measures for hierarchical routing, the forwarding of notifications has to be extended such that notifications are always propagated towards the root broker (cf. change 1 below) and the forwarding of subscriptions has to be restricted such that subscriptions are only forwarded upwards in the broker tree (cf. change 2 below). Furthermore, since subscriptions are not propagated downwards in the broker tree, only subscriptions towards the root broker influence the size of the routing tables (cf. change 3 below). A consequence of this is that, for example, a broker has only remote routing entries for its child brokers but no entries for its parent broker. Incorporating these three changes into the equations presented is rather simple:

- Change 1: $P^{n}\left(B_{i} B_{j}\right)$ is defined as before, but defined to be equal to 1 if $B_{j}$ is the parent of $B_{i}$.

- Change 2: $P^{s}\left(B_{i} B_{j}\right)$ is defined as before, but defined to be 0 if $B_{j}$ is not the parent broker of $B_{i}$.

- Change 3: $X^{s}\left(B_{i} B_{j}\right)$ is defined as before, but defined to be 0 if $B_{j}$ is the parent broker of $B_{i}$.

Following, we present an exemplary setting with hierarchical routing in order to demonstrate the applicability of the stochastic model and to obtain some quantitative results that illustrate the analysis method and provide insight into the mathematical relationships. To this end, a scenario was chosen that illustrates trade-offs in using different broker topologies and provides some quantitative results that can be easily interpreted by the reader. For 


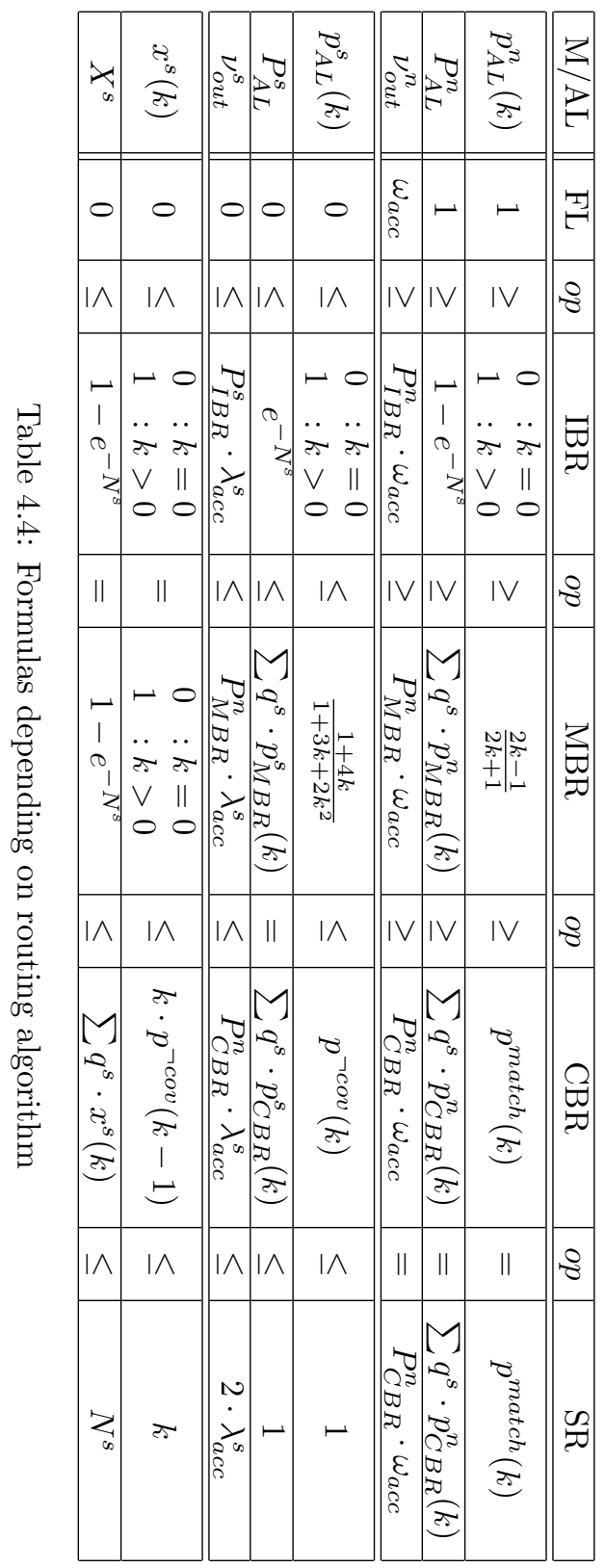




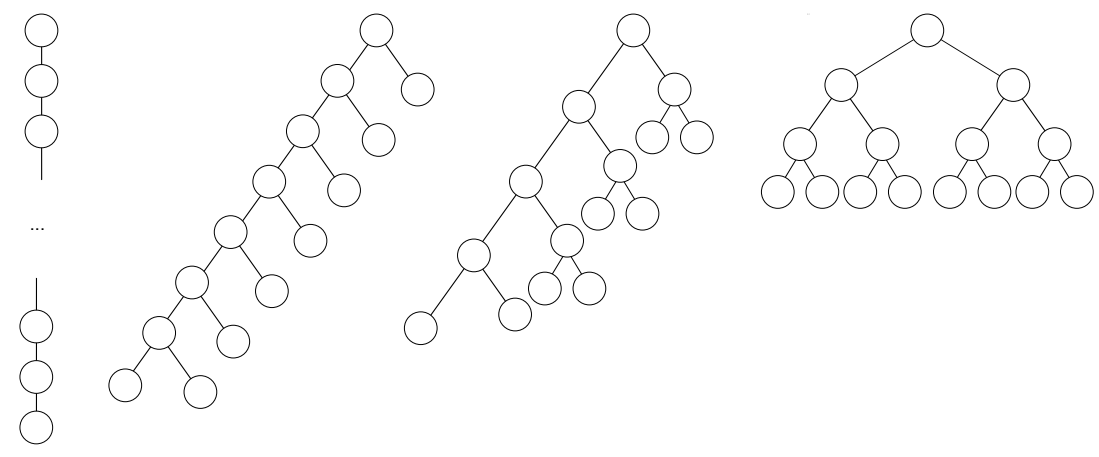

Figure 4.24: Resulting topologies for 15 brokers $\left(T_{1}, T_{2}, T_{4}, T_{8}\right)$

the exemplary setting, brokers that are connected by overlay links are also directly connected by physical links. Results that consider the physical topology explicitly are presented in Chapter 5 in the context of the case study. We consider seven exemplary topologies consisting of 127 brokers arranged in a binary tree. Since they are acyclic, all of them have 126 links. The trees are varied from a balanced tree with seven levels to a linear arrangement of the brokers with 127 levels by restricting the maximum number of brokers at each level of the tree to $1,2,4,8,16,32,64$, respectively. We denote the topologies by $T_{1}, T_{2}, T_{4}, T_{8}, T_{16}, T_{32}$ and $T_{64}$. Figure 4.24 shows the four topologies that would result for 15 brokers.

Besides using different topologies, most experiments we present in the context of the example vary the mean overall number of subscriptions $N^{s}$ in the system from 10 to 700,000 where the subscriptions are equally distributed among brokers. Furthermore, we use not only one but 1000 different types for filter classes the identity-based hierarchical routing is based on. This means that a notification is matched by a subscription if they both contain the same filter class. The mean lifetime of subscriptions $\mu^{s}(B)^{-1}$ is $60 s$ for all brokers and filter classes and an overall publication rate $\omega$ is set to $(1000 s)^{-1}$, i.e., 1000 notifications are published per second equally distributed among brokers and filter classes.

Routing Table Sizes. Figure 4.25 shows the expected overall number of remote routing entries $X^{s}$ (which is the sum of the routing table sizes of all brokers) for the exemplary seven topologies plotted against the mean number of subscriptions in the system. As the number of subscriptions grows, $X^{s}$ increases strictly monotonically with the gradient ${ }^{4}$ continuously decreasing. As expected, $X^{s}$ eventually converges to 126,000 for all topologies. The gradient at which the individual plot of a certain topology starts in the origin depends on the average path length of the topology. The longer the average path length, the steeper the plot is for lower number of subscriptions and the flatter it is for higher number of subscriptions. In consequence, with $T_{1}$ the system is saturated for much lower number of subscriptions than, for example, with $T_{64}$. An

\footnotetext{
tions.

${ }^{4}$ Please note that the following figures use a logarithmic scale for the number of subscrip-
} 


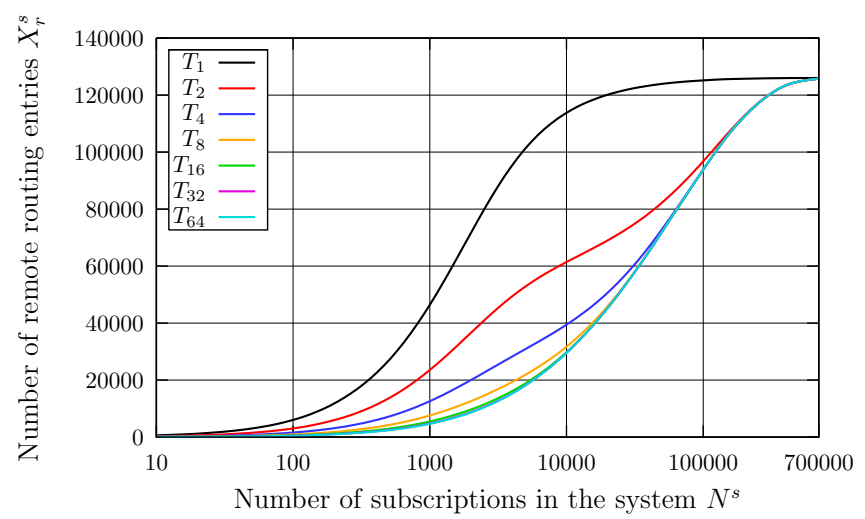

Figure 4.25: Routing table sizes

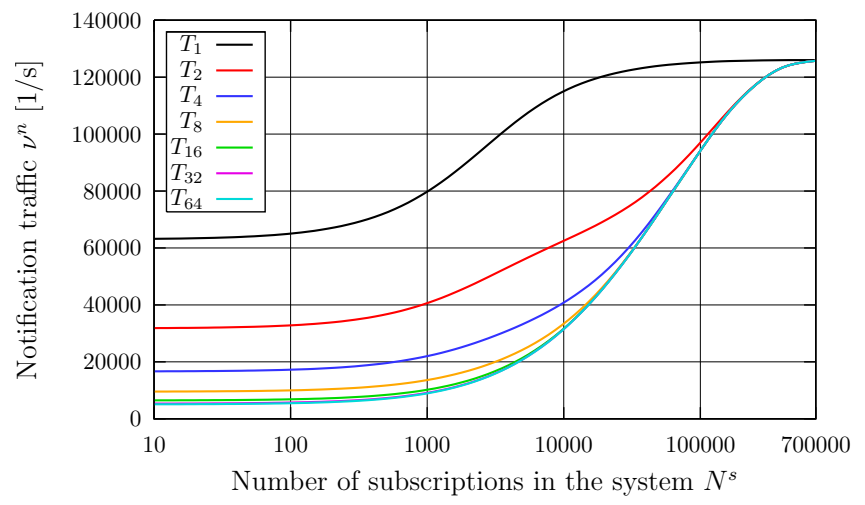

Figure 4.26: Notification traffic

interesting effect can be discovered for the plot of $T_{2}$ : it has an extra inflection point which is caused by the asymmetry of $T_{2}$. This effect is also visible for $T_{4}$ although it is less obvious in this case.

Notification Traffic. Figure 4.26 shows the overall notification traffic $\nu^{n}$ for the exemplary setting. For all topologies the traffic monotonically increases until it converges to 126, 000 notifications per second. For smaller numbers of subscriptions, the traffic is dominated by the upward traffic towards the root broker. Since the path to the root broker is shorter for more balanced topologies, the curves for these topologies begin at a lower notification traffic. For a higher number of subscriptions, the traffic is dominated by the downward traffic finally ending with flooding of the entire overlay network.

Subscription Traffic. Figure 4.27 shows the subscription traffic $\nu^{s}$ in the publish/subscribe system for the seven exemplary topologies. For all topologies the subscription traffic rises from 0 to a global maximum, then starts to drop, and finally converges to 0 as the number of subscriptions $N^{s}$ is further 


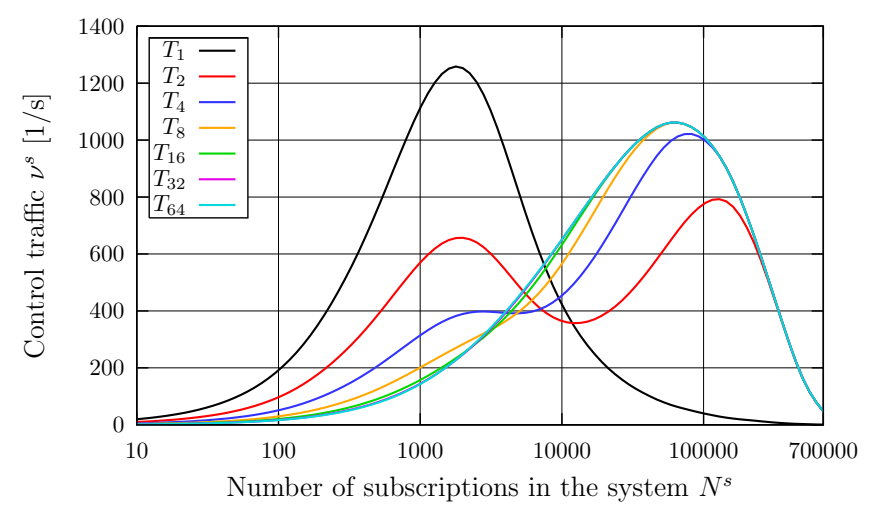

Figure 4.27: Control traffic

increased. This is because for small numbers of subscriptions, the routing tables are only lightly filled and, therefore, when a subscription is issued or revoked the probability that some subscription messages are generated is high. Thus, in the beginning, as we increase the number of subscriptions the subscription traffic increases. However, as the routing tables get increasingly filled, a point is reached after which the probability of generating a subscription message when a subscription is issued or revoked starts to drop. This results in less and less subscription messages being generated. In consequence, the subscription traffic starts to decrease after reaching its maximum and eventually converges to 0 even though the number of subscriptions and, thus, also the number of those that are issued or revoked per second further increases.

For $T_{1}$, the subscription traffic reaches its maximum for a much smaller number of subscriptions than in the other topologies. This is due to the fact that in this topology the routing tables fill up more quickly. The first subscription of a filter class occupies on average 63 routing entries. Thus, for small numbers of subscriptions, the broker subscription traffic is high. However, given that the routing tables fill up much more quickly, the subscription traffic passes its advance and decline cycle much earlier.

The plots of $T_{2}$ and $T_{4}$ are different in that they additionally have a local maximum caused by the asymmetry of these topologies. For example, consider topology $T_{2}$. A single subscription at the broker in the lower left corner causes half of the routing entries of its filter class to be occupied, while for the other half at least 63 subscriptions are needed. This shows that $T_{2}$ effectively consists of two parts whose routing tables fill up with different speeds when the number of subscriptions rises. The part that fills up much more quickly is, thus, responsible for the local maximum. The same is true for $T_{4}$ although the effect is not that incisive.

Comparison with Notification Flooding and Simple Routing. With flooding no remote routing entries are used: Each published notification is sent once over every overlay link. Figure 4.28 shows the saved notification traffic $\tilde{\nu}^{n}$ for the seven exemplary topologies. The saved traffic equals 126, 000 notifications per second if there are no subscriptions in the system. For larger numbers 


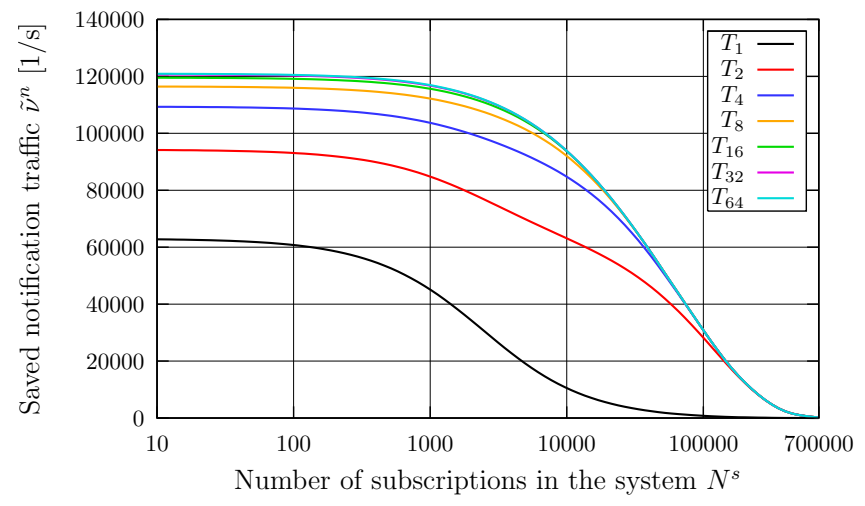

Figure 4.28: Saved traffic compared to FL

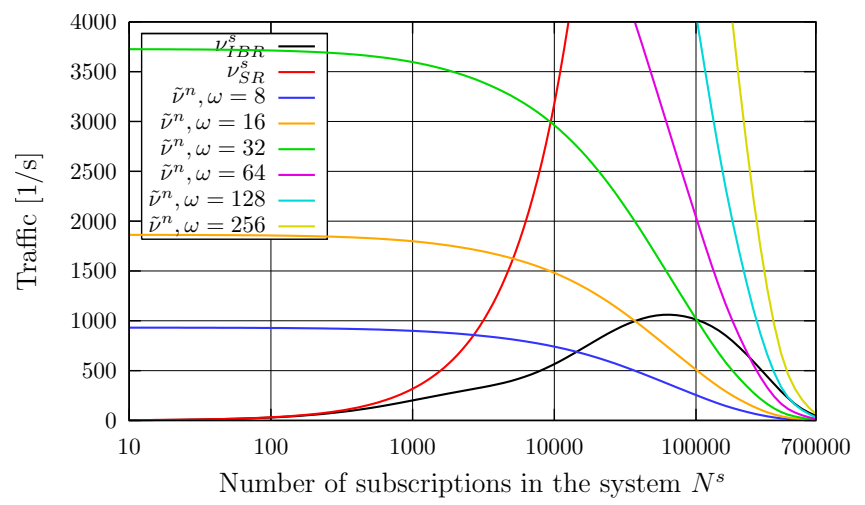

Figure 4.29: Traffic for $T_{8}$ 


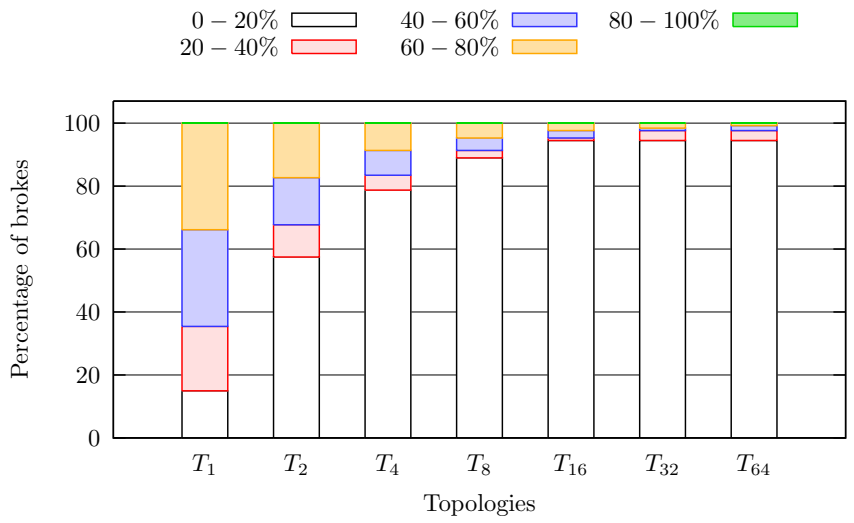

Figure 4.30: Distribution of broker load

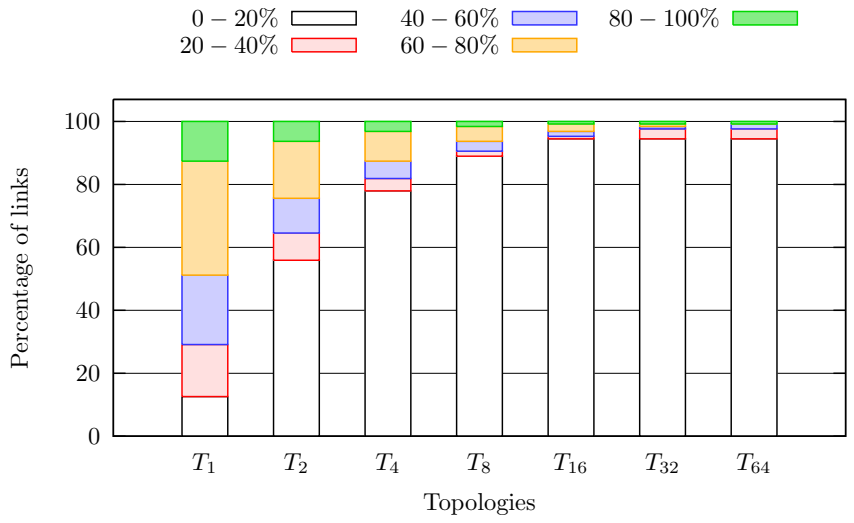

Figure 4.31: Distribution of link load

of subscriptions, the saved traffic continuously decreases and finally converges to 0 . The more unbalanced the topology is, the steeper the drop in the saved traffic is. For $T_{1}$, the saved traffic approaches 0 much faster, while for the other topologies it differs mainly for smaller numbers of subscriptions.

For the last example, we include a comparison with simple routing, where each subscription is always propagated towards the root broker. In this case, the notification traffic is the same but the subscription traffic is $\nu_{S R}^{s}$. Figure 4.29 depicts the subscription traffic $\nu_{I B R}^{s}$ and $\nu_{S R}^{s}$ as well as the saved notification traffic $\tilde{\nu}^{n}$ for topology $T_{8}$ and different publication rates $\omega$. It shows that depending on $\omega$, filtering may perform worse than flooding if the number of subscriptions in the system exceeds a certain threshold. For example, if $\omega=32 s^{-1}$ this is the case for identity-based routing if $N^{s}>100,000$ and for simple routing if $N^{s}>10,000$. Identity-based routing outperforms simple routing.

Broker and Link Load. Figure 4.30 shows the distribution of the broker load for the exemplary topologies and $N^{s}=1000$. For the calculation we set a broker capacity of $c(B)=1400$ messages per second for all individual brokers. 


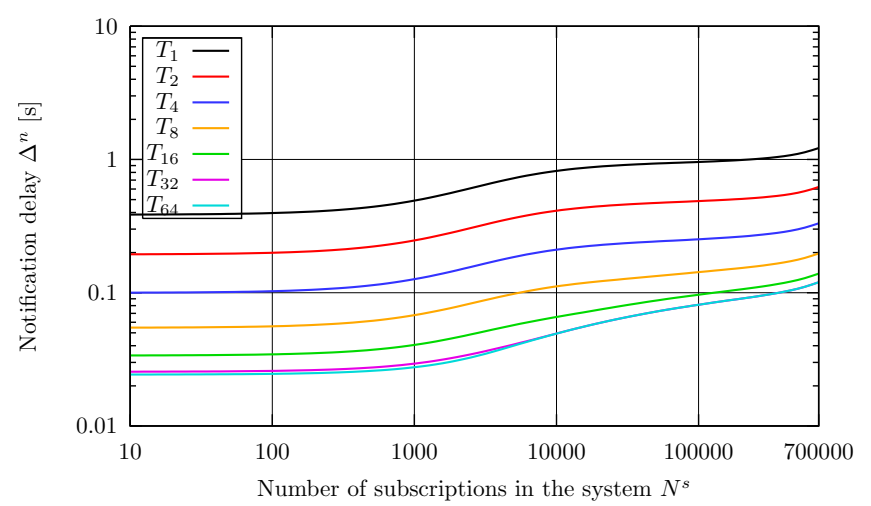

Figure 4.32: Maximum expected notification delay (for $\omega=1000 \mathrm{~s}^{-1}$ )

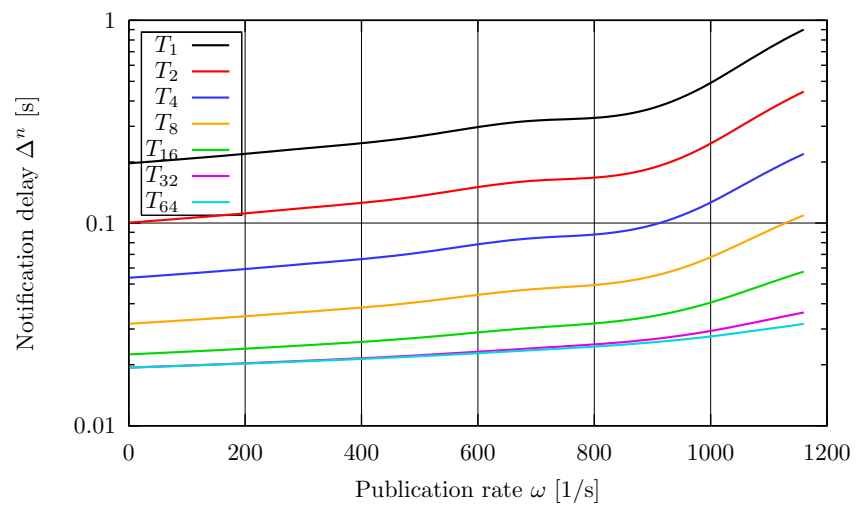

Figure 4.33: Maximum expected notification delay (for $N^{s}=1000$ )

It can be seen from the figure that the topology has a major impact on the broker load distribution. The more unbalanced the topology is, the higher is the proportion of the brokers which are heavily utilized. For example, for $T_{1}$ the majority of brokers has an utilization of more than $40 \%$, while for $T_{64}$ more than $90 \%$ of the brokers have a utilization of less than $20 \%$ and only a few brokers near to the root broker are heavily utilized.

Figure 4.31 shows the distribution of the link load for the exemplary topologies and $N^{s}=1000$. Again, it can be seen that the more unbalanced topologies perform worse than the more balanced topologies. For the calculation we set a link capacity of $c(\bar{l})=1200$ messages per second for all individual links.

Message Delays. Figure 4.32 shows the maximum expected delay for the seven exemplary topologies and plotted against the expected number of subscriptions in the system. As we can see, the more balanced the topology, the smaller the notification delay. The more skewed the topology, the faster the notification delay rises as the number of subscriptions goes up.

Figure 4.33 shows the maximum expected notification delay for $N^{s}=1000$ subscriptions and increasing overall publication rate. For all seven topologies, 


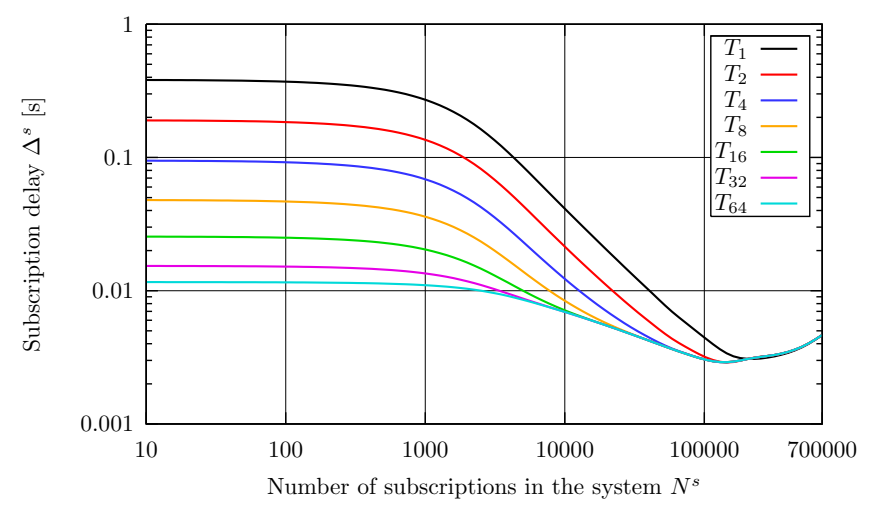

Figure 4.34: Maximum expected subscription delay (for $\omega=1000 s^{-1}$ )

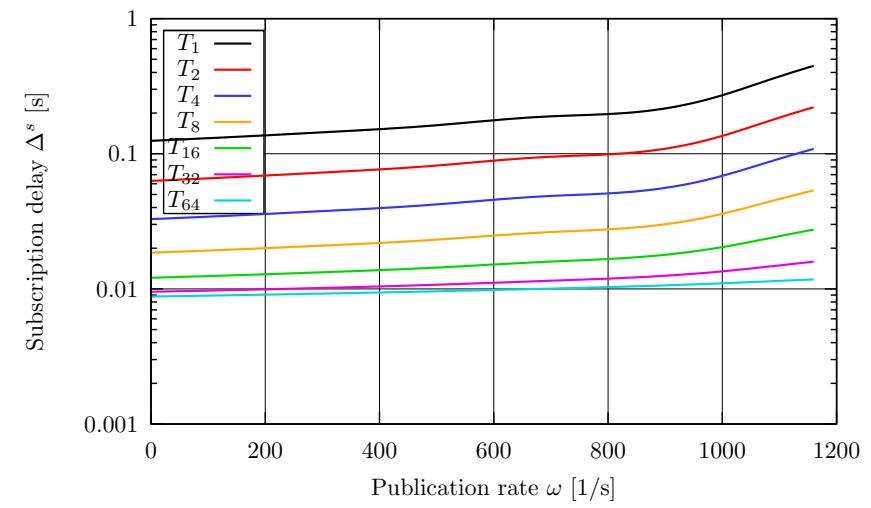

Figure 4.35: Maximum expected subscription delay (for $N^{s}=1000$ )

the notification delay increases slowly up to a publication rate of 800 and starts increasing at a higher rate after that with the plot being steeper for more skewed topologies. Again, as expected, topology $T_{1}$ has the largest worst path notification delay.

Figure 4.34 shows the maximum expected subscription delay for the seven exemplary topologies plotted against the expected number of subscriptions in the system. Again, the more balanced the topology, the smaller the delay for low number of subscriptions. For all topologies, the subscription delay drops to a minimum as the number of subscriptions rises. This is expected since a point is reached after which every broker has at least one local subscription for every filter class and, therefore, new subscriptions become almost immediately active. From then on, the waiting time in the message queues of brokers dominates the subscription delay. Thus, after the minimum the delay increases until the broker capacity is reached. Then the system will be overloaded and the delay will diverge to infinity.

Figure 4.35 shows the maximum expected subscription delay for $N^{s}=1000$ subscriptions and increasing overall publication rate. Again, for all seven topologies, the notification delay increases slowly up to a publication rate of 800 and 


\begin{tabular}{|c|c|c|c|c|}
\hline $\mathbf{F}_{i} / j$ & FList $_{* 1}$ & FList $_{* 2}$ & $\ldots$ & FList $_{d *}$ \\
\hline$\overline{\mathbf{F}_{1}=F \text { List }_{1 *}}$ & $\overline{\overline{\left[a_{11}, b_{11}\right]}}$ & $\overline{\left[a_{12}, b_{12}\right]}$ & $\ldots$ & $\overline{\left[a_{1 d}, b_{1 d}\right]}$ \\
\hline $\mathbf{F}_{2}=$ FList $_{2 *}$ & $\overline{\left[a_{21}, b_{21}\right]}$ & $\overline{\left[a_{22}, b_{22}\right]}$ & $\ldots$ & $\overline{\left[a_{2 d}, b_{2 d}\right]}$ \\
\hline $\mathbf{F}_{k}=F$ List $_{k *}$ & {$\left[a_{k 1}, b_{k 1}\right]$} & {$\left[a_{k 2}, b_{k 2}\right]$} & $\ldots$ & {$\left[a_{k d}, b_{k d}\right]$} \\
\hline
\end{tabular}

Table 4.5: Filter list FList

starts increasing at a higher rate after that with the plot being steeper for more skewed topologies.

\subsection{Generalizations and Extensions}

In Section 4.4, we made some restrictions due to the compactness of representation of the publish/subscribe system model and to not disturb the readability. In this section, we remove these restrictions and extend the model to be more applicable to real-world scenarios. The presented generalizations are threefold: First, we extend the data and filter model by allowing multidimensional attributes and filters (Section 4.6.1). Then, in Section 4.6.2 we extend the latter result by allowing randomly chosen numbers of attributes and filters. Finally, we explain how we can support a more sophisticated behavior in the message forwarding and processing time (Section 4.6.3).

\subsubsection{Multidimensional Notifications/Subscriptions}

In Section 4.4.1 we restricted the number of attributes in notifications and the number of attribute filters in subscriptions to one. Following, we extend this model to support multiple dimension as we already described in Chapter 2. In a first step, we generalize the definitions for matching, covering and overlapping operations of Section 4.4.2 to the multidimensional case.

Matching. From Section 4.4.2 we already know when an interval $I$ matches a notification with the attribute value $x$ : matches $(I, x)$. In the one-dimensional case, the filter matching equals the interval matching. If there are more dimensions we have to extend the definition. Now, a $d$-dimensional filter $\mathbf{F}=\left[I_{1}, \ldots I_{d}\right]$ matches a $d$-dimensional notification $\mathbf{n}=\left[x_{1}, \ldots, x_{d}\right]$ iff each attribute filter in $\mathbf{F}$ matches the corresponding attribute in $n$. Formally, we get:

$$
\begin{aligned}
\operatorname{matches}(\mathbf{F}, \mathbf{n}) & \Leftrightarrow n \in \mathbf{F} \\
& \Leftrightarrow \forall i: \text { matches }\left(I_{i}, x_{i}\right)
\end{aligned}
$$

This approach leads to a more complex filter list FList (cf. Section 4.4.2). Now, an arbitrary filter list $F$ List $=\left(F\right.$ List $\left._{i j}\right)$ can be displayed as a matrix as depicted in Table 4.5. The depicted filter list consists of $k$ filters arranged in rows which can be accessed by $F$ List $_{i *}=\mathbf{F}_{i}$. Each column shows the attribute filters for all filters $\left(F L i s t_{* j}\right)$ for the dimension $j$. The filter list is a logical 
disjunction of all contained filters FList $=\mathbf{F}_{1} \vee \ldots \vee \mathbf{F}_{k}$ and, therefore, creates a not necessarily connected subspace/subset of $\mathbb{R}^{d}$. As in the one-dimensional case a filter list matches a notification if at least one filter matches it. Thus,

$$
\begin{aligned}
\text { matches }(\text { FList }, \mathbf{n}) & \Leftrightarrow \mathbf{n} \in \text { FList } \\
& \Leftrightarrow \exists i: \text { matches }\left(\mathbf{F}_{i}, \mathbf{n}\right) \\
& \Leftrightarrow \exists i: \forall j:\left[\left(a_{i j} \leq x_{j} \leq b_{i j}\right) \vee\left(a_{i j} \geq x_{j} \geq b_{i j}\right)\right]
\end{aligned}
$$

Covering. Similarly to the approach in matching, we have to redefine the covering operation of filters, which now depends on all $d$ intervals contained by the filters:

$$
\begin{aligned}
\operatorname{covers}\left(\mathbf{F}_{i}, \mathbf{F}_{j}\right) & \Leftrightarrow \mathbf{F}_{i} \supseteq \mathbf{F}_{j} \\
& \Leftrightarrow \forall k: \operatorname{covers}\left(I_{i k}, I_{j k}\right)
\end{aligned}
$$

A filter list FList as depicted in Table 4.5 covers a filter $\mathbf{F}$, iff at least one filter of F List covers F. Formally,

$$
\begin{aligned}
\operatorname{covers}(F \text { List }, \mathbf{F}) & \Leftrightarrow F \text { List } \supseteq \mathbf{F} \\
& \Leftrightarrow \exists k: \operatorname{covers}\left(F \operatorname{List}_{k *}, \mathbf{F}\right) \\
& \Leftrightarrow \exists k: \forall l: \operatorname{covers}\left(I_{k l}, I_{l}\right)
\end{aligned}
$$

For the subscription forwarding decision the inverse relation is used. If a filter $\mathbf{F}$ is not covered by filter list FList, the subscription containing $\mathbf{F}$ is forwarded. Thus,

$$
\begin{aligned}
\neg \operatorname{covers}(\text { F List }, \mathbf{F}) & \Leftrightarrow \neg \exists k: \forall l: \operatorname{cover} s\left(I_{k l}, I_{l}\right) \\
& \Leftrightarrow \forall k: \neg \forall l: \operatorname{covers}\left(I_{k l}, I_{l}\right)
\end{aligned}
$$

Overlapping. Similarly to the approaches before, we have to redefine the overlap operation of filters, which now depends on all $d$ intervals contained by the filters:

$$
\begin{aligned}
\operatorname{overlaps}\left(\mathbf{F}_{i}, \mathbf{F}_{j}\right) & \Leftrightarrow \neg\left(\mathbf{F}_{i} \cap \mathbf{F}_{j}\right)=\emptyset \\
& \Leftrightarrow \forall k: \operatorname{overlaps}\left(I_{i k}, I_{j k}\right)
\end{aligned}
$$

Finally, a (subscription) filter list FList overlaps a filter $\mathbf{F}$ (of a subscription), iff at least one filter of F List overlaps F. Formally,

$$
\begin{aligned}
\operatorname{overlaps}(F L i s t, \mathbf{F}) & \Leftrightarrow(F \text { List } \cap \mathbf{F})=\emptyset \\
& \Leftrightarrow \exists k: \operatorname{overlaps}\left(F \operatorname{List}_{k *}, \mathbf{F}\right) \\
& \Leftrightarrow \forall k: \neg \forall l: \operatorname{overlaps}\left(I_{k l}, I_{l}\right)
\end{aligned}
$$

Having these modified definitions, we are able to generalize the probabilities for matching, covering and overlapping resp. the related probabilities for the forwarding of notifications, subscriptions and advertisements. The new formulas substitute the ones derived in Section 4.4.7. 
Matching Probability for Simple and Covering-based Routing. Based on the definition in Equation 4.88, we can determine $P($ matches $(F \bar{L} i s t, \mathbf{n}))$, the probability that a $d$-dimensional notification $\mathbf{n}=\left[x_{1}, \ldots, x_{d}\right]$ is matched (covered) by any of $k d$-dimensional subscriptions. Thus,

$$
\begin{aligned}
P_{C B R}^{\text {match }}(\mathbf{n}, k) & =P(\text { matches }(F \overline{L i s t}, \mathbf{n})) \\
& =1-\left[1-\prod_{j=1}^{d} P\left(\text { matches }\left(F \overline{\text { List }}{ }_{j}, x_{j}\right)\right)\right]^{k} \\
& =1-\left[1-2^{d} \cdot \prod_{j=1}^{d} F^{s}\left(x_{j}\right) \cdot\left(1-F^{s}\left(x_{j}\right)\right)\right]^{k}
\end{aligned}
$$

This result contains Equation 4.31 as a special case for $d=1$. Assuming that the probability distributions of the limits $F^{s}(\cdot)$ are known, $P($ matches $(F \bar{L} i s t, \mathbf{n}))$ finally only depends on the notification $\mathbf{n}$ and on the variable $k$ which is the number of filters. The variable $P_{C B R}^{\text {match }}(\mathbf{n}, k)$ is itself a random variable based on the state space which equals all possible values of $\mathbf{n}$. The expected matching probability of a notification whose attribute values are distributed according to the probability density function $f^{n}(\mathbf{n})$ when $k$ subscriptions are active is then:

$$
\begin{aligned}
p_{S R}^{\text {match }}(k, d) & =E\left[P_{C B R}^{\text {match }}(\mathbf{n}, k)\right] \\
& =\int_{\mathbf{n}} f^{n}(\mathbf{n}) \cdot P_{C B R}^{\text {match }}(\mathbf{n}, k) d \mathbf{x} \\
& =\int_{\mathbf{n}} f^{n}(\mathbf{x}) \cdot\left(1-\left[1-2^{d} \prod_{i=1}^{d} F_{i}^{s}\left(x_{i}\right)\left(1-F_{i}^{s}\left(x_{i}\right)\right)\right]^{k}\right) d \mathbf{x}
\end{aligned}
$$

This result contains Equation 4.34 as a special case $(d=1)$. The probability density function of the attributes $f^{n}(\mathbf{n})$ is multidimensional. Therefore, dependencies of attributes can be modeled with this approach. In case that all attributes are independent, $f^{n}(\mathbf{n})$ can be expressed by the product of $d$ probability density functions $f_{i}^{n}\left(x_{i}\right)$ which can simplify the calculation of $p^{\text {match }}(k, d)$.

Matching Probability for Merging-based Routing. In case of mergingbased routing we merge each dimension separately following the approach described for one dimension in Section 4.4.2. Thus, a notification is matched by a filter list iff $\forall j:$ matches $\left(F\right.$ List $\left._{j}, x_{j}\right)$, i.e., the notification must be matched by the filter list in all dimensions independently. The probability for matching one dimension matches $\left(F L_{i s t}, x_{j}\right)$ was already calculated in Equation 4.37. Thus, the matching probability for merging-based routing which equals the forwarding probability can be generalized in the following way:

$$
\begin{aligned}
& P_{M B R}^{\text {match }}(\mathbf{n}, k)=\prod_{i=1}^{d}\left(1-\left[F_{i}^{s}\left(x_{i}\right)\right]^{2 k}-\left[1-F_{i}^{s}\left(x_{i}\right)\right]^{2 k}\right) \\
& p_{M B R}^{\text {match }}(k, d)=\int_{\mathbf{n}} f^{n}(\mathbf{x}) \cdot P_{M B R}^{\text {match }}(\mathbf{n}, k) d \mathbf{x}
\end{aligned}
$$




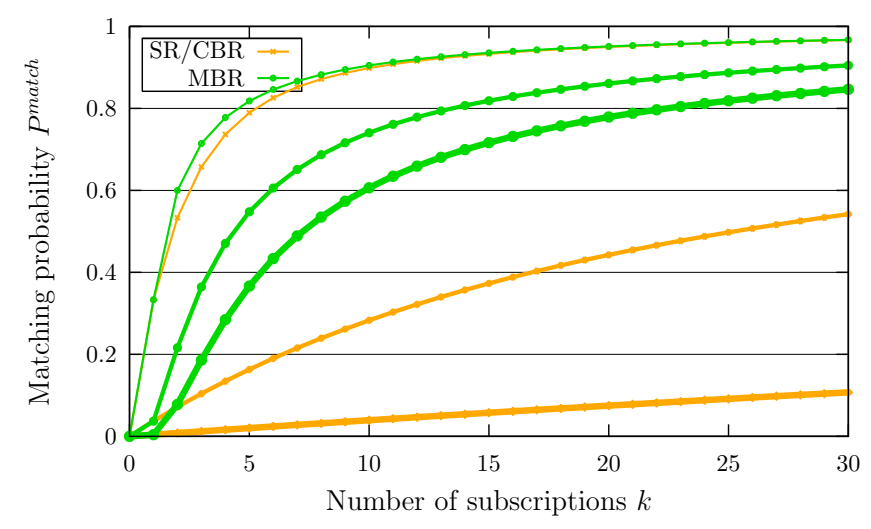

Figure 4.36: Matching probability for multiple dimensions

This general formula again contains the special case of Equation 4.37 in Section 4.4.7. Comparing the latter formulas for SR/CBR and MBR one can see that for $k=1$ the matching probabilities are equal $\left(p_{S R}^{\text {match }}(1, d)=p_{M B R}^{\text {match }}(1, d)\right)$. This equality can be proven by showing that:

$$
\prod_{i=1}^{d} 2 \cdot F_{i}^{s}\left(x_{i}\right)\left(1-F_{i}^{s}\left(x_{i}\right)\right)=\prod_{i=1}^{d}\left(1-\left[F_{i}^{s}\left(x_{i}\right)\right]^{2}-\left[1-F_{i}^{s}\left(x_{i}\right)\right]^{2}\right)
$$

To show the influence of the number of dimensions, we plotted the probability $P^{\text {match }}(\mathbf{n}, k)$ for a simplified scenario and an interval limit distribution of $F^{s}(x)=x$ within the interval $[0,1]$ in Figure 4.36. The thickness of line correlates with the number of dimensions where $d \in\{1,3,5\}$. For all values of $d$ and for both algorithms the curves show a similar behavior which starts at $P^{\text {match }}(\mathbf{n}, 0)=0$ and tends to $P^{\text {match }}(\mathbf{n}, k>>1)=1$. For all cases with a fixed $k$, a higher number of dimensions decreases the probability for matching. This is because, each dimension has a matching probability that is less than $100 \%$. Since all dimensions have to match each additional dimension reduces the overall matching probability. However, the influence of the number of dimensions on the probability is weaker for MBR than for SR/CBR. Similar to the one-dimensional case, this fact can be explained by the generalization behavior of MBR. If in each dimension the matching probability for MBR is higher than for $\mathrm{SR} / \mathrm{CBR}$, also the overall matching probability is greater.

Probability for not Covering in Covering-based Routing. After focusing on notifications we move on to subscription forwarding. In general, an incoming subscription is forwarded if it is not covered by any of $k$ other active subscriptions. Using Equation 4.91, we can directly derive the probability that a given $d$-dimensional filter $\mathbf{F}$ is not covered by a filter list FList consisting of $k d$-dimensional filters: 


$$
\begin{aligned}
P_{C B R}^{\neg c o v}(\mathbf{a}, \mathbf{b}, k) & =P_{C B R}(\neg \operatorname{covers}(F \text { List }, \mathbf{F})) \\
& =\left(1-2^{d} \prod_{i=1}^{d}\left[F_{i}^{s}\left(a_{i}\right)\left(1-F_{i}^{s}\left(b_{i}\right)\right)\right]\right)^{k} \\
p_{C B R}^{\neg c o v}(k, d) & =\int_{\mathbf{a}} \int_{\mathbf{b}} f^{s}(\mathbf{a}, \mathbf{b}) \cdot P_{C B R}^{\neg c o v}(\mathbf{a}, \mathbf{b}, k) \mathrm{d} \mathbf{b} \mathrm{d} \mathbf{a}
\end{aligned}
$$

The first equation is a generalization of Equation 4.47. Since all $a_{i}$ and $b_{i}$ are themselves random variables, we can determine the expected value (cf. Equation 4.99). In this equation, $f^{s}(\mathbf{a}, \mathbf{b})$ is a $2 d$-dimensional probability density function that can be expressed as $f^{s}(\mathbf{a}, \mathbf{b})=\prod_{i=1}^{d} f_{i}^{s}\left(a_{i}, b_{i}\right)$ in case of independent interval dimensions.

Probability for not Covering in Merging-based Routing. In mergingbased routing a filter does not cover a filter list iff $\forall j: \neg \operatorname{covers}\left(F L i s t_{j}, \mathbf{F}_{j}\right)$, i.e. that for each dimension the filter is not covered. The probability for $\neg \operatorname{covers}\left(F L i s t_{j}, \mathbf{F}_{j}\right)$ was already calculated in Equation 4.52 . Thus, the probability for not covering in merging-based routing which equals the forwarding probability can be generalized in the following way:

$$
\begin{aligned}
P_{M B R}^{\neg c o v}(\mathbf{a}, \mathbf{b}, k) & =P_{M B R}(\neg \operatorname{covers}(F \operatorname{List}, \mathbf{F})) \\
& =\prod_{i=1}^{d}\left(1-\left[\left(1-F_{i}^{s}\left(a_{i}\right)\right]^{2 k}-\left[F_{i}^{s}\left(b_{i}\right)\right]^{2 k}+\left[F_{i}^{s}\left(b_{i}\right)-F_{i}^{s}\left(a_{i}\right)\right]^{2 k}\right]\right) \\
p_{M B R}^{\neg c o v}(k, d) & =\int_{\mathbf{a}} \int_{\mathbf{b}} f^{s}(\mathbf{a}, \mathbf{b}) \cdot P_{M B R}^{\neg c o v}(\mathbf{a}, \mathbf{b}, k) \mathrm{d} \mathbf{b} \mathrm{d} \mathbf{a}
\end{aligned}
$$

For $k=1$ the expected probabilities for not covering filters in CBR/SR and MBR are equal $\left(p_{C B R}^{\neg c o v}(1, d)=p_{M B R}^{\neg c o v}(1, d)\right)$. It can be proven by showing that:

$$
1-2 \cdot F_{i}^{s}\left(a_{i}\right) \cdot\left(1-F_{i}^{s}\left(b_{i}\right)\right)=1-\left(F_{i}^{s}\left(a_{i}\right)\right)^{2}+\left(F_{i}^{s}\left(b_{i}\right)\right)^{2}-\left(F_{i}^{s}\left(b_{i}\right)-F_{i}^{s}\left(a_{i}\right)\right)^{2}
$$

In Figure 4.37 the probability for not covering subscriptions $P^{\neg c o v}(\mathbf{a}, \mathbf{b}, k)$ of covering-based routing and merging-based routing is shown for 1, 3 and 5 dimensions. The thickness of the lines symbolizes the number of dimensions. For CBR an increased number of dimensions leads to a fast convergence of the probability to $100 \%$ even for a small number of subscriptions $k$. This stems from the fact that each dimension makes it more unlikely that a subscription is covered by others and, therefore, the probability for not covering is increased. For MBR this effect is smaller than in CBR since in each dimension it is more likely that a new subscription is already covered by actives ones.

Advertisement-based Routing. For advertisement-based routing we can build on the overlapping definition in Equation 4.93 that allows us to derive the overlapping probability $P($ overlaps $(F$ List, $\mathbf{F}))$ of a $d$-dimensional filter $\mathbf{F}$ and 


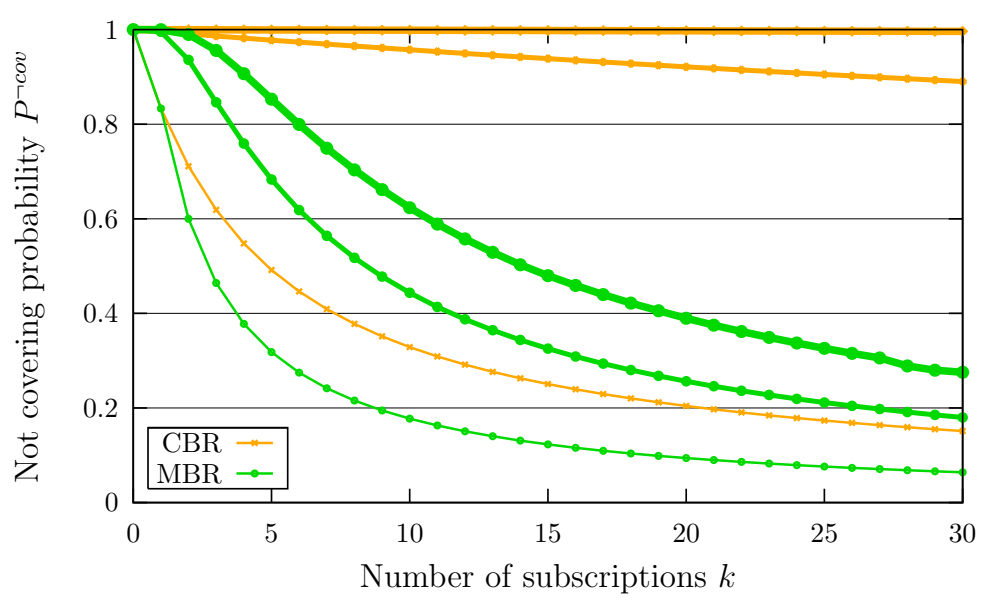

Figure 4.37: Probabilities for not covering for multiple dimensions

a given filter list F List consisting of $k$ filters. The resulting formula depends on $k$ and the interval limits of all filters. Thus, we write $P^{\text {olap }}(\mathbf{a}, \mathbf{b}, k)$ for the core overlapping probability formula. This allows us to derive a generalized form of Equation 4.56 and a general expression for the forwarding probability of subscriptions in advertisement-based routing:

$$
\begin{aligned}
& P^{\text {olap }}(\mathbf{a}, \mathbf{b}, k)=1-\left[1-\prod_{i=1}^{d}\left(1-\left(F_{i}^{s}\left(a_{i}\right)\right)^{2}-\left(1-F_{i}^{s}\left(b_{i}\right)\right)^{2}\right)\right]^{k} \\
& p_{A+A L}^{s}\left(k^{s}, k^{a}\right)=\int_{\mathbf{a}} \int_{\mathbf{b}} f^{s}(\mathbf{a}, \mathbf{b}) \cdot P^{o l a p}\left(\mathbf{a}, \mathbf{b}, k^{a}\right) \cdot P_{A L}^{\neg c o v}\left(\mathbf{a}, \mathbf{b}, k^{s}\right) \mathrm{d} \mathbf{b d} \mathbf{a}
\end{aligned}
$$

The latter formula for $p_{A+A L}^{s}\left(k^{s}, k^{a}\right)$ contains $P_{A L}^{-c o v}$ which depends on the algorithm chosen for subscription forwarding (AL) like SR, IBR, CBR or MBR. In Figures 4.16, 4.17 and 4.18 of Section 4.4 .7 we already pointed out the superposition of both parameters on $p_{A+A L}^{s}\left(k^{s}, k^{a}\right)$. Therefore and for the compactness of representation, in Figure 4.38 we show the influence of the number of dimensions in filters on the overlapping probability. This results equals to the forwarding probability in case of SR. For all numbers of dimensions 1,3,5, the overlapping probability starts at $0 \%$ for 0 active advertisements, increases monotonically and converges then to $100 \%$ for large number of advertisements. The figure confirms the intuition that the increase is slower when the number of dimensions increases. This is caused be the fact that each additional dimension requires a subscription to overlap in one more attribute. This fact also explains why the curves do not intersect despite at $k=0$.

\subsubsection{Variable Number of Attributes}

So far we had a model with an arbitrary but fixed number of attributes in notifications and filters in subscriptions and advertisements. In real world scenarios users and clients of publish/subscribe systems often have the freedom to choose the number of attributes and attribute filters in a flexible way. For example, a 


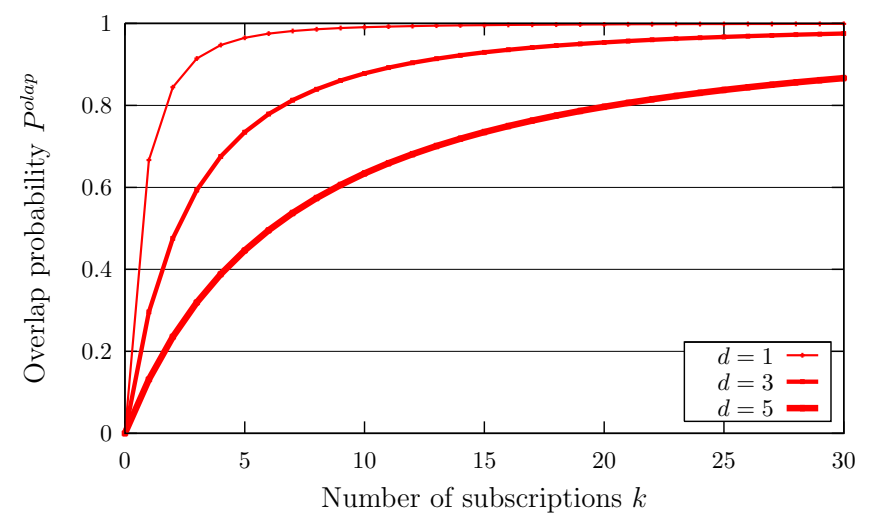

Figure 4.38: Overlapping probabilities for multiple dimensions

search mask of a website contains all possible attributes to restrict the results to get. Nevertheless, the user is allowed to fill in only a subset of the provided attribute filters. Therefore, we need a way to provide more flexibility to the model. Following, we focus on the forwarding probability of notifications. In this model notifications and subscriptions contain up to $d$ ordered attributes resp. attribute filters. The concrete number of attributes of a notification is $k^{n}$ and of attribute filters of a subscription is $k^{s}$. In all cases, if $k^{s}>k^{n}$ the forwarding probability is $0 \%$ because there are more restrictions on the attributes then attributes exists in the notification. For other cases $\left(k^{n} \geq k^{s}\right)$ we have to know how likely they are. This probability is $P\left(k^{n}, k^{s}\right)$. For $k^{n} \geq k^{s}$ the matching probability $p^{\text {match }}$ only depends on $k^{s}$ and the number of subscriptions. This is, because only the first $k^{s}$ attributes of notifications must be considered. Thus, we can derive the following formula:

$$
\begin{aligned}
p^{n}(n) & =\sum_{k^{s}=1}^{d} \sum_{k^{n}=k^{s}}^{d} P\left(k^{n}, k^{s}\right) \cdot p^{\text {match }}\left(n, k^{s}\right) \\
& =\sum_{k^{s}=1}^{d} p^{\text {match }}\left(n, k^{s}\right) \cdot \sum_{k^{n}=k^{s}}^{d} P\left(k^{n}, k^{s}\right) \\
& =\sum_{k^{s}=1}^{d} p^{\text {match }}\left(n, k^{s}\right) \cdot P\left(k^{n} \geq k^{s}\right)
\end{aligned}
$$

In the last derivation step we use an accumulated probability $P\left(k^{n} \geq k^{s}\right)$ that is the probability for having equal or more notification attributes than attribute filters $k^{s}$.

As an example, we uniformly select $k^{n}$ and $k^{s}$ between 1 and 3 (which is $d$ then). There are nine combinations of the dimensions of notifications and subscriptions. Thus, it hold $P\left(k^{n}, k^{s}\right)=1 / 9$. The probability that $k^{n}$ is greater than $k^{s}=\{1,2,3\}$ is $3 / 9,2 / 9$ and $1 / 9$. Substituted into the latter formula we get:

$$
p^{n}(n)=1 / 3 \cdot p^{\text {match }}(n, 1)+2 / 9 \cdot p^{\text {match }}(n, 2)+1 / 9 \cdot p^{\text {match }}(n, 3)
$$


The probabilities $p^{\text {match }}(n, d)$ can be determined using the formulas in the previous section. Thus, the determination of the forwarding probability of notifications for a variable number of attributes is deduced to already known formulas. For the covering probability a very similar approach is possible. Note that we assume here that the attributes are selected from the left to the right in an ordered way. That means for $k^{s}=1$ only the first dimension is chosen and not an arbitrary one. To support such cases the model must be further extended.

\subsubsection{Utilization and Delay}

Following we explain how to calculate utilization and delay for an extended model of brokers and physical links. To be as general as possible we introduce $S^{n}(B)$ and $S^{s}(B)$ which are the service time distributions for both types of messages at broker $B$. For the compactness of the representation we leaf out advertisements here. This model for the service time distributions can be extended to include the matching overhead, which depends on the routing table size of the respective broker. This can, e.g., be done by weighting the service time by the routing table size. We assume that the processing of notifications and subscriptions is done independently leading to two queues with two service units.

Physical links and routers are modeled as $M / G / 1$ instead of $M / M / 1$ queueing systems. For physical links, we additionally introduce the latency $\delta(\bar{l})$ of link $\bar{l}$. It is caused by the physical delay of the link due to limited signal propagation speed, which is determined by the length of the cable and whether electrical or optical transmission is used. For routers, we set $\delta(\bar{l})=0$. Finally, we define $S(\bar{l})$ as the service time distribution of $\bar{l}$ for all types of messages. First, we calculate the overall utilization of broker $B$ caused by the notifications and control messages (cf. Equation 4.78) as follows:

$$
U^{x}(B)=\nu_{i n}^{x}(B) \cdot E\left[S^{x}(B)\right]
$$

Similar to Section 4.4.8, we use the placeholder $x$ for notifications and subscriptions to not repeat structurally identical formulas. Note that if $U^{x}(B)>1$ for some broker, the system is overloaded and not in a steady state. When modeling the brokers with $M / G / 1$ instead of $M / M / 1$ queues (cf. Equation 4.79), we can approximate the mean waiting time $W^{x}(B)$ of a message at broker $B$ using the Pollaczek-Khinchin mean value formula [53]:

$$
W^{x}(B)=\frac{\nu_{i n}^{x}(B) \cdot \operatorname{Var}\left[S^{x}(B)\right]}{2\left(1-U^{x}(B)\right)}
$$

In the equation above, $\operatorname{Var}\left[S^{x}(B)\right]$ is the second centralized moment of the service time distribution $S^{x}(B)$ of broker $B$. The approximated expected delay for a notification $D^{n}(B)$ and for a control message $D^{s}(B)$ at broker $B$ is then given by:

$$
D^{x}(B)=W^{x}(B)+E\left[S^{x}(B)\right]
$$

Since we can use any service time distribution as long as its second moment $\operatorname{Var}\left[S^{x}(B)\right]$ and the expected processing times $E\left[S^{x}(B)\right]$ can be computed, we are able to model graduated broker delays for the different message 
types. For example, we could use a hyper-exponential service time distribution consisting of two exponential distributions, one for the notifications and another for the control messages. Following, the utilization $U(\bar{l})$ of the physical component $\bar{l}$ is determined (cf. Equation 4.80):

$$
U(\bar{l})=\nu_{\text {out }}(\bar{l}) \cdot E[S(\bar{l})]
$$

Then, we can approximate the mean waiting time $W(\bar{l})$ of a message at component $\bar{l}$ with:

$$
W(\bar{l})=\frac{\nu_{\text {out }}(\bar{l}) \cdot E\left[S(\bar{l})^{2}\right]}{2(1-U(\bar{l}))}
$$

The latter Equation contains the same Pollaczek-Khinchin mean value formula as in Equation 4.106. By adding waiting time, service time and latency we get the expected delay of a component. The approximated expected delay for a notification $D^{n}(\bar{l})$ and for a control message $D^{s}(\bar{l})$ at the physical component $\bar{l}$ is then given by (cf. Equations 4.81):

$$
\begin{aligned}
D^{n}(\bar{l}) & =W(\bar{l})+E\left[S^{n}(\bar{l})\right]+\delta(\bar{l}) \\
D^{s}(\bar{l}) & =W(\bar{l})+E\left[S^{s}(\bar{l})\right]+\delta(\bar{l})
\end{aligned}
$$

Having these formulas we are able to determine the notification and the subscription message delay as described in Section 4.4.9.

\subsection{Discussion}

In this chapter, we presented a stochastic system model for publish/subscribe systems. It is the first model that covers all major aspects of distributed contentbased publish/subscribe systems. It extends existing work on modeling of publish/subscribe systems in several important aspects: (i) all major performance and cost-relevant measures of publish/subscribe systems are considered, (ii) a large variety of different peer-to-peer and hierarchical content-based routing algorithms is supported, (iii) the proposed models cover a wider range of systems and can be used for comprehensive analysis. We built the mathematical analysis on the identification of required inputs and outputs of the model. As input we defined the environment including client behavior and the physical network. As outputs we defined important performance and cost measures for publish/subscribe systems, such as routing table sizes, traffics, utilizations and, last but not least, the delay of notifications and subscriptions. With the help of probability and queueing theory we stepwise derived formulas for the outputs. An important insight of this derivation was the definition of probabilities for matching, covering and overlapping and for the forwarding of messages. The presented model has a modular design and, thus, can easily be extended to meet new requirements. For example, a relocation of publishers as described in [27] can be mapped to a shift of publication rates from one broker to another. Many other extension in fields of the data and filter model, the physical network and the broker behavior are possible.

During the derivation of the model and in the evaluation section we demonstrated the effectiveness and practicality of the model. We explained how it can be used for a detailed qualitative and quantitative analysis. The special 
focus here was on routing algorithms, which are a crucial parameter of publish/ subscribe systems. We could show the parameter range for the usage of each mentioned algorithm. In fact, this finding is the trigger to think about adaptive routing algorithms that we will present in Chapter 6 of this thesis. Summarizing, the proposed model can be used to identify and eliminate performance bottlenecks before a system is deployed as well as for provisioning and capacity planning. Moreover, the ability to predict the system performance is important to ensure that applications meet their Quality of Service requirements. Finally, performance prediction helps to optimize a publish/subscribe system and find an optimal broker overlay topology. How this is done we explain in the following Chapter 5 .

Although we presented some model extension, such as the multidimensional data and filter model, every system model and also the presented stochastic model abstracts from reality by leaving out details. Such details which are not important at the first glace may lead to uncertainties of the model output compared with the real system. In reality we always have to deal with this problem because the real world is very complex and changes over time. The problem in fields of publish/subscribe was and still is the lack of real publish/ subscribe system data. Authors do either use synthetic workloads based on assumptions they qualitatively explain or they use real data from non-publish/ subscribe systems, such as search engines $[46,106]$ and interpret resp. convert them into notifications and subscriptions. While the notifications are relatively straight forward to generate content-based filter generation for subscriptions is often not more consistent than just generate them completely synthetically. Another missing point which is not addressed with this approach is the local distribution of clients within the publish/subscribe overlay. Thus, the research focus must be twofold, exploration of new real-world data sources such as social networks, and, the adaption of the model towards robustness which allows an appropriate performance predication even under uncertainties.

Another open research question is the correlated client behavior, where the arrival processes of notifications and subscriptions are no longer independent. Currently, we can capture correlated behavior only partially by considering equivalent quasi-stationary settings. For example, if notifications $n_{1}$ and $n_{2}$ are expected to be published once an hour and 600 times an hour, but all occurrences of $n_{2}$ directly follow that of $n_{1}$ within ten minutes, we can capture this situation by setting the publication rate of $B$ to $1 s^{-1}$ instead of $(6 s)^{-1}$.

Nevertheless, due to the modular design of the presented model we are sure that many further requirements and extension can be integrated easily. 


\title{
Chapter 5
}

\section{Capacity Planning}

\author{
Contents \\ 5.1 Introduction .................. 99 \\ 5.2 Related Work . . . . . . . . . . . . . 100 \\ 5.3 Efficient Calculation of the Model . . . . . . . . 100 \\ 5.3.1 General Workflow for Model Calculation . . . . . . . . . . . 101 \\ 5.3 .2 Numerical Challenges . . . . . . . . . . . . . . . 103 \\ 5.3 .3 Efficiency . . . . . . . . . . . . . . . 106 \\ 5.4 Optimized Configurations ............ 107 \\ 5.4.1 Optimizing Publish/Subscribe Infrastructures . . . . . . . . 107 \\ 5.4 .2 Optimization Complexity . . . . . . . . . . . 108 \\ 5.5 Case Study . . . . . . . . . . . . . 110

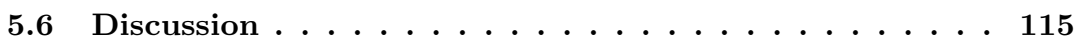

\subsection{Introduction}

In Chapter 4, a stochastic model of publish/subscribe systems was introduced that we now use for capacity planning, a process to find optimized configurations. As mentioned in Section 4.4, there are four input parameters of publish/ subscribe systems. Beside two parameters that are fixed, namely the behavior of clients and the physical network, there are two parameters, namely the topology of the overlay network and the applied routing algorithm that the designer can manually set when putting a system into production. It is easy to see that with the help of the analytical model, the knowledge about the physical network and the expected client behavior, a system designer can investigate the influence of the free parameters on the performance and cost measures. This way, a designer is able to manually look for good configurations that provide an acceptable performance. Since this is a cumbersome task, it seems reasonable to use an optimizer resp. an optimizing algorithm to find optimized configurations for the publish/subscribe system. This is what we call capacity planning and what we deal with in this chapter. 
The foundation of capacity planning is an efficient calculation of the model for a single point in the parameter space. This issue will be discussed in Section 5.3 right after a discussion about the related work in Section 5.2. With this result we go to Section 5.4, where we describe how the central part of the capacity planning strategy, namely the optimizer, is integrated into the life cycle of a publish/subscribe system (cf. Section 3.4). Furthermore, we discuss the complexity of the given optimization problem. Finally, in Section 5.5 we present a case study which emphasizes how the presented approach can be used to determine an optimized configuration for a given realistic scenario. We end up with a discussion in Section 5.6.

\subsection{Related Work}

The foundation of a capacity planning is a applicable system model predicting the system behavior. As we have seen in the related work section of the previous chapter, many models either do not provide sufficient details or they are based on simulations that need a long time to determine results. Thus, lots of the discussions in Section 4.2 is also relevant for capacity planning.

There is only a few publication that explicitly refer to capacity planning of publish/subscribe systems. In Pankajakshan [72] the optimal placement of brokers within the physical network is tackled. This is done by mapping optimal message flows in publish/subscribe systems to a multi-commodity transportation problem. Solutions are determined by using heuristics. This interesting mapping approach suffers from its pure publish/subscribe system model which is assumed to be topic based. Furthermore, some configuration parameters such as the routing algorithm are not supported.

Another capacity planning approach for event-based systems is the one of Rathfelder et al. [83]. In opposite to our approach that is on message routing level, their focus is on a higher system level which models interactions and communication between components in event-driven applications. For capacity planning the paper proposes an extended Palladio Component Model (PCM) [51] which includes the implementation of system components, the performance of external components, the deployment platform, and the system usage profile. PCM supports the automatic transformation of these architecturelevel performance models to predictive performance models including layered queueing network or queueing Petri net or simulation code. Unfortunately, all these predictive models require time- and resource consuming runs to get results. Thus, it cannot be used for large-scale capacity planning. Due to this limitations, the authors do capacity planning only for limited scenarios, and, instead, describe the automation approach and the accuracy of the model.

\subsection{Efficient Calculation of the Model}

In Chapter 4 we introduced a model consisting of around 80 formulas that describe the behavior of a distributed content-based publish/subscribe system. For the provided plots, the calculation of the results was not a problem since we had a relatively limited number of parameters to vary. A vast amount of time was used to validate the analytic results by discrete event simulations. In this 


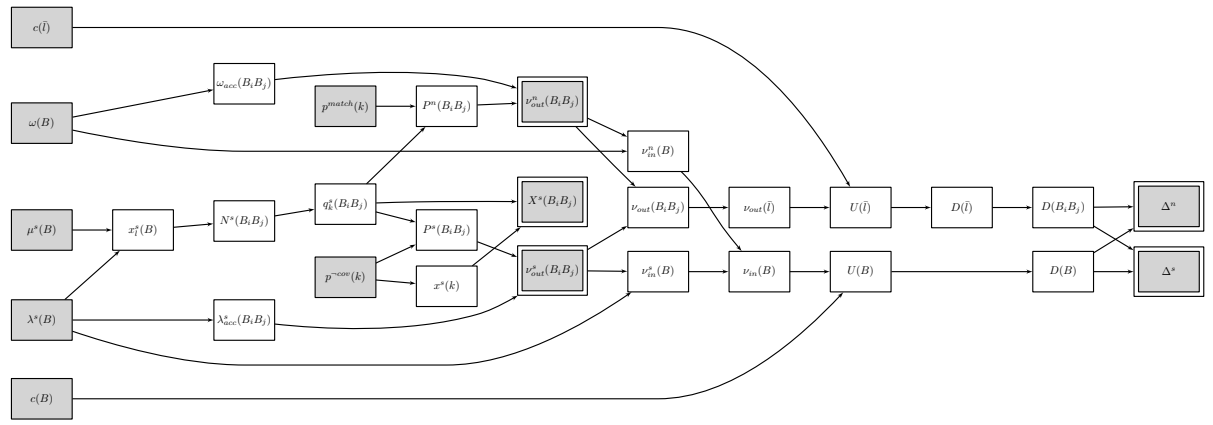

Figure 5.1: Dependencies between quantities

chapter, we want to optimize the configuration of a publish/subscribe system which requires to calculate the model far more often. The complexity of the optimization, which is exactly determined in Section 5.4.2, forces us to focus on the efficient calculation of the model. In a first step, we analyze the dependencies of the formulas of the system model. These functional dependencies generate a complex network depicted in Figure 5.1. In opposite to Figure 4.21(a) of the analysis chapter, we are not interested in the monotony of the functional dependencies. But as before, the figure shows how starting from the inputs on the left hand-side the outputs on the right side are determined. Unfortunately, the figure is not the solution to efficiently calculate the model, because it does not depict the dependencies on the routing algorithm and on the overlay and underlay network. Such additional dependencies occur if the formula of a quantity is recursively defined indicated by symbols such as $\mathcal{S}, \mathcal{P}$ resp. $\mathcal{T}$ or by routing dependent subscripts for probabilities such as $p_{A L}^{n}$ and $p_{A L}^{s}$. The latter two quantities are connected to other challenges in the calculation of the model because they could contain integrals as well as infinite series. Following, we provide a general workflow to calculate the complete system model, i.e., the outputs for arbitrary input parameters (Section 5.3.1). To complete the model calculation in Section 5.3.2, we provide solutions for some remaining numerical challenges in the formulas. Finally, in Section 5.3.3 we show which speedup is reached by calculating the introduced model instead of using a discrete event simulation.

\subsubsection{General Workflow for Model Calculation}

Before presenting a workflow, to calculate the model we need some formal definition based on an unrooted tree $\mathbb{G}=(\mathbb{V}, \mathbb{E})$ with vertices $v_{i}$ and directed edges $e_{i j}$ :

Definition 1 (Leaf function $\mathbb{L}$ ). The leaf function $\mathbb{L}$ applied to an unrooted tree $\mathbb{G}$ maps to all leaves of $\mathbb{G}$. It is given by $\mathbb{L}(\mathbb{G})=\left\{v_{i}: \operatorname{deg}\left(v_{i}\right)=1\right\}$. $\operatorname{deg}\left(v_{i}\right)$ is the degree of vertex $v_{i}$.

Definition 2 (Leaf edge function $\overrightarrow{\mathbb{L}}$ ). The leaf edge function $\overrightarrow{\mathbb{L}}$ applied to an unrooted tree $\mathbb{G}$ maps to all edges $e_{i j}$ whose destination vertex is a leaf of $\mathbb{G}$. It is given by $\overrightarrow{\mathbb{L}}(\mathbb{G})=\left\{e_{i j}\right.$ : $\left.\operatorname{deg}\left(v_{j}\right)=1\right\}$. 

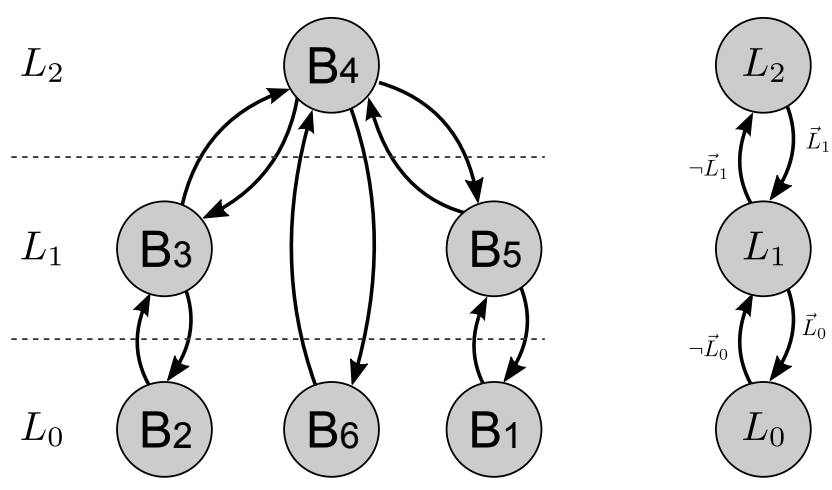

Figure 5.2: Leaves on different levels

Definition 3 (Inverted edge function $\neg \overrightarrow{\mathbb{L}}$ ). The inverted edge function $\neg \overrightarrow{\mathbb{L}}$ applied to a set of edges $\mathbb{E}$ inverts all edges. It is given by $\neg \overrightarrow{\mathbb{L}}(\mathbb{E})=\left\{e_{i j}: e_{j i} \in \mathbb{E}\right\}$.

Definition 4 (Trim function $\mathbb{T}$ ). The trim function $\mathbb{T}$ applied to an unrooted tree $\mathbb{G}$ maps to a subgraph of $\mathbb{G}$ which is generated by the removal of its leafs $\mathbb{L}(\mathbb{G})$. It is given by $\mathbb{T}(\mathbb{G})=\mathbb{G} \backslash \mathbb{L}(\mathbb{G})$. We implicitly require that edges that were connected to at least one vertex from $\mathbb{G}$ are also removed.

Definition 5 (Level-n leaf function $\left.\mathbb{L}_{n}\right)$. The level-n leaf function $\mathbb{L}_{n}(n \in \mathbb{N})$ applied to an unrooted tree $\mathbb{G}$ maps to all leaves of $\mathbb{G}$ after it has been trimmed $n$ times. It is given by $\mathbb{L}_{n}(\mathbb{G})=\mathbb{L}\left(\mathbb{T}(\ldots \mathbb{T}(\mathbb{G}))_{n}\right)$.

Definition 6 (Level-n leaf edge function $\overrightarrow{\mathbb{L}}_{n}$ ). The level-n leaf edge function $\overrightarrow{\mathbb{L}}_{n}$ applied to an unrooted tree $\mathbb{G}$ maps to all leaf edges $e_{i j}$ of $\mathbb{G}$ after it has been trimmed $n$ times. It is given by $\overrightarrow{\mathbb{L}}_{n}(\mathbb{G})=\overrightarrow{\mathbb{L}}\left(\mathbb{T}(\ldots \mathbb{T}(\mathbb{G}))_{n}\right)$.

Obviously, it holds $\mathbb{L}_{0}(\mathbb{G})=\mathbb{L}(\mathbb{G})$. Furthermore, for increasing $n$ the number of level-n leaves $\left|\mathbb{L}_{n}\right|$ is monotonically decreasing until it reaches 0 . The number of available levels is $l$ if for all $n>l-1$ it holds $\left|\mathbb{L}_{n}\right|=0$ and $\left|\mathbb{L}_{l-1}\right|>1$. The number of levels depends on the diameter of $\mathbb{G}$ which is in the range $0 \leq$ $d(\mathbb{G}) \leq|\mathbb{G}|-1$. If $d(\mathbb{G})$ is odd, the graph has $(d(\mathbb{G})-1) / 2$ levels and 1 vertex in the highest level. If $d(\mathbb{G})$ is even, the graph consists of $d(\mathbb{G}) / 2$ levels and two vertices in the highest level. Of course, it holds that the sum of all level-n leaves equals the number of vertexes $\sum_{i=0}^{l-1}\left|\mathbb{L}_{i}\right|=|\mathbb{V}|$.

As an example in Figure 5.2(a) different leaf levels of a tree consisting of six vertices (brokers) are shown. Vertices with the same level are arranged in the same horizontal position. In this case, the graph has $l=3$ levels with the following level-n leaves: $\mathbb{L}_{0}=\left\{B_{1}, B_{2}, B_{6}\right\}, \mathbb{L}_{1}=\left\{B_{3}, B_{5}\right\}$ and $\mathbb{L}_{2}=\left\{B_{4}\right\}$. In Figure 5.2(b), we summarized $\mathbb{L}_{0}, \mathbb{L}_{1}$ and $\mathbb{L}_{2}$. The connecting edges (overlay links) correspond to the level-n leaf edges and its inversions: $\overrightarrow{\mathbb{L}}_{0}=\left\{B_{3} B_{2}, B_{4} B_{6}, B_{5} B_{1}\right\}$, $\overrightarrow{\mathbb{L}}_{1}=\left\{B_{4} B_{3}, B_{4} B_{5}\right\}$ and $\neg \overrightarrow{\mathbb{L}}_{0}=\left\{B_{2} B_{3}, B_{6} B_{4}, B_{1} B_{5}\right\}, \neg \overrightarrow{\mathbb{L}}_{1}=\left\{B_{3} B_{4}, B_{5} B_{4}\right\}$.

Figure 5.1 and the sets of $\overrightarrow{\mathbb{L}}_{i}$ and $\neg \overrightarrow{\mathbb{L}}_{i}$ are the foundation of the workflow to calculate the entire system model. The following pseudocode enumerates the major steps: 
1. Determine all $\overrightarrow{\mathbb{L}}_{i}$ and $\neg \overrightarrow{\mathbb{L}}_{i}$.

2. Apply the initial values to brokers $B$ and physical links $\bar{l}: \omega(B), \lambda^{s}(B)$, $\mu^{s}(B), x_{l}^{s} B, c(B), c(\bar{l})$.

3. Iterate through $\overrightarrow{\mathbb{L}}_{0}, \ldots, \overrightarrow{\mathbb{L}}_{n}, \neg \overrightarrow{\mathbb{L}}_{n}, \ldots, \neg \overrightarrow{\mathbb{L}}_{0}$. For all overlay links in the current set $B_{i} B_{j}$ calculate values that depend on $\mathcal{S}\left(B_{i} B_{j}\right)$ or $\mathcal{P}\left(B_{i} B_{j}\right)$ : $N^{s}\left(B_{i} B_{j}\right), \lambda_{\text {acc }}^{s}\left(B_{i} B_{j}\right), \lambda_{\text {acc }}^{s}\left(B_{i} B_{j}\right), P^{n}\left(B_{i} B_{j}\right), P^{s}\left(B_{i} B_{j}\right), \nu_{\text {out }}^{s}\left(B_{i} B_{j}\right)$.

4. Iterate through all brokers and calculate broker related measures: $\nu_{\text {out }}^{n}(B)$, $\nu_{i n}^{n}(B), \nu_{\text {out }}^{s}(B), \nu_{\text {in }}^{s}(B)$. En passant $\nu_{\text {out }}^{n}\left(B_{i} B_{j}\right), \nu^{n}, \nu^{s}$ and $\nu$ are calculated.

5. Iterate through all brokers and calculate broker related measures: $U(B)$ and $D(B)$.

6. Iterate through all overlay links and calculate the traffic on underlay links, i.e., $\nu_{\text {out }}^{n}(\bar{l})$ and $\nu_{\text {out }}^{s}(\bar{l})$.

7. Iterate through all underlay links and calculate the utilization and delay on these links, i.e., $U(\bar{l})$ and $D(\bar{l})$.

8. Calculate the notification delay $\Delta^{n}$ and subscription delay $\Delta^{s}$. Therefore, the maximum over all brokers have to be determined as described in Section 4.4.9.

The notification flow $\nu_{\text {out }}^{n}\left(B_{i} B_{j}\right)$ cannot be calculated in step (3) since its calculation depends on both $\mathcal{S}\left(B_{i} B_{j}\right)$ and $\mathcal{P}\left(B_{i} B_{j}\right)$. Therefore, it is calculated in step (4) when all necessary values are available. If there is only one filter class, steps (4) and (5) can be joined together. If there are several filter classes, there are distinct values for $\omega(B), \lambda^{s}(B)$ and $\mu^{s}(B)$ for each class. Thus, steps (2) to (4) are repeated for all filter classes. The resulting traffics for each filter class of step (4) are summed up and the workflow is continued the usual way from step (5) to (8).

\subsubsection{Numerical Challenges}

With providing the latter workflow, the calculation of the model seems to be effectively computable. This is true for most of the formulas (cf. Chapter 4) that often contain only basic mathematical operators. Nevertheless, there are two remaining numerical challenges that have to be solved, namely infinite series and integrals that cannot be solved analytically. Following, we describe how we dealt with these challenges.

Poisson Distribution. In Equation 4.2 and 4.3, we provided formulas for the expected number of subscriptions resp. advertisements when they are Poisson distributed. The general form is given by: $q_{k}=\frac{e^{-N}}{k !} \cdot N^{k}$. This formula contains a major challenge which is the fast growing terms: $k$ ! and $N^{k}$. Assuming a double precision floating point variable these calculations exceed the limit for $k>170$. One way out of the dilemma would be to use arbitrary-precision arithmetics. Another is to split the calculation: $q_{k}=e^{-N} \cdot \prod_{i=1}^{k} \frac{N}{i}$. The factors of the product are calculated step by step keeping the result small. Both ways are algorithmic 


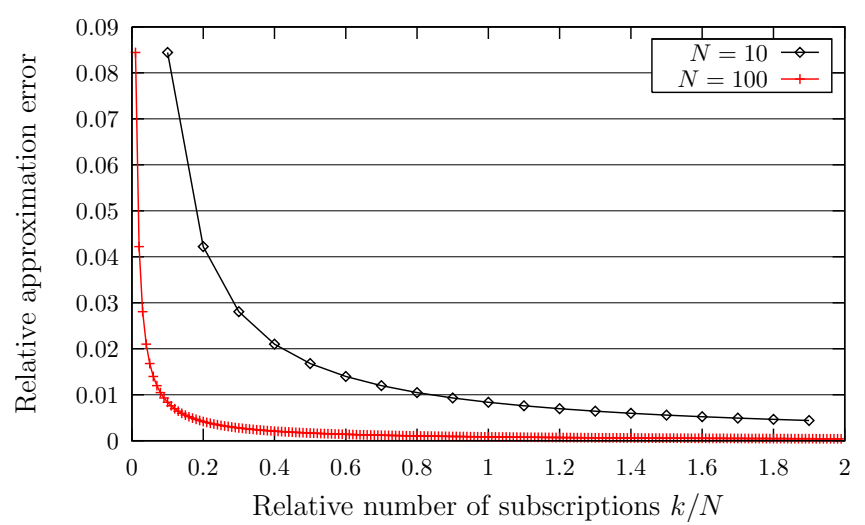

Figure 5.3: Error of $q_{k}$

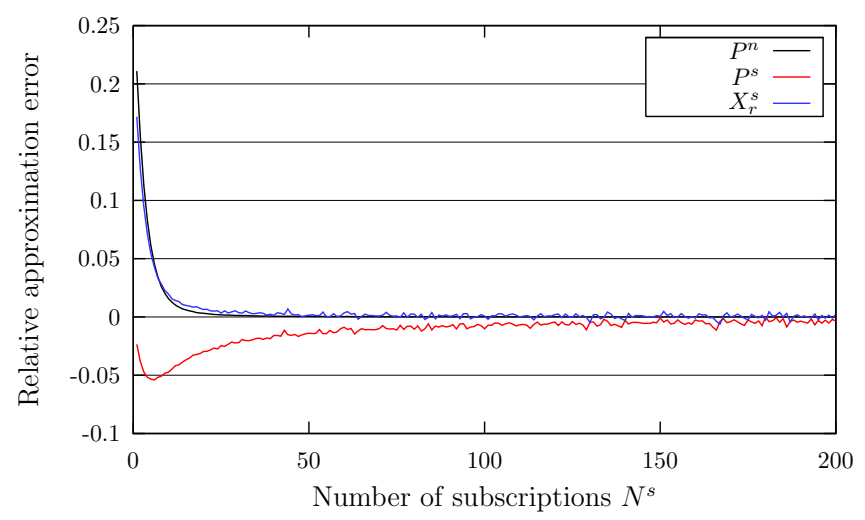

Figure 5.4: Error for $P^{n}, P^{s}$ and $X^{s}$ 
ways and require more computational effort for higher precision. However, there are also ways to go based on mathematical conversions. By applying $e^{\ln (\cdot)}$ we get $q_{k}=e^{k \cdot \ln (N)-\ln (k !)-N}$. The remaining term $k !$ can further kept small by substituting the identity $\ln (k !)=\ln (k)+\ln (k-1)+\ldots+\ln (2)$. Another way for large values of $k$ is to use Stirling's approximation $k ! \cong \sqrt{2 \pi k}\left(\frac{k}{e}\right)^{k}$ :

$$
\begin{aligned}
q_{k} & =e^{k \cdot \ln (N)-\sum_{i=2}^{k} \ln (i)-N} \\
& \cong e^{k \cdot \ln (N)-0.5 \cdot \ln (2 \pi)-(k+0.5) \cdot \ln (k)+k-N}
\end{aligned}
$$

The relative approximation error of Stirling's approximation for $N=10$ and $N=100$ is plotted in Figure 5.3. Obviously, the approximation is rather good especially for great values of $N$. The latter formulas do never occur in a single calculation but it is embedded into other more complex equations, like Equations 4.20, 4.27, 4.28 and 4.41. Since these formulas calculate the expected value, they all contain infinite series of the following general form: $F(N)=\sum_{k=0}^{\infty} q_{k}(N) \cdot f(k)$. This formula contains the before mentioned Poisson distribution $q_{k}$ and a variable part $f(k)$ which is either $x^{s}(k), x^{a}(k), p^{n}(k), p^{s}(k)$ or $p^{a}(k)$. To avoid the infinite summation of the terms, we limit the calculation of the sum. We empirically found out that it is sufficient to determine only the first $K$ terms where $K=\operatorname{Max}(50,3 N)$. Due to the exponential drop of $q_{i}(N)$ for $i>N$ and the very good-natured behavior of $f(i)$, it is guaranteed that all higher summands of the infinite sum do only have a marginal impact on the result. To reduce the still high number of summands for large $N$, we found out that $F(N) \cong f(N)$. In Figure 5.4 we show the comparison of this approximation compared to the summation described before for $P^{n}, P^{s}$ and $X^{s}$. Due to these results, we decided to switch the calculation of these values to the approximation $f(N)$ for $N>100$. Then, even for $P^{s}$ the deviation is approximately $1 \%$.

Integrals. In the stochastic model integrals occur when we calculate probabilities for matching, not covering or overlapping. In all formulas we calculated the expected value of continuously distributed values of attributes $x_{i}$ resp. filters $a_{i}$ and $b_{i}$. In concrete terms, we have Equations 4.95, 4.97, 4.99 and 4.101 that do have a general form like:

$$
\begin{aligned}
p_{A L}^{\text {match }}(k, d) & =\int_{\mathbf{x}} f^{n}(\mathbf{x}) \cdot P_{A L}^{\text {match }}(\mathbf{x}, k, d) d \mathbf{x} \\
p_{A L}^{\neg \operatorname{cov}}(k, d) & =\int_{\mathbf{a}} \int_{\mathbf{b}} f^{s}(\mathbf{a}, \mathbf{b}) \cdot P_{A L}^{\neg \operatorname{cov}}(\mathbf{a}, \mathbf{b}, k, d) \mathrm{d} \mathbf{b} \mathrm{d} \mathbf{a}
\end{aligned}
$$

Both formulas consist of an integral with a variable integrand. Even the simplest integral for matching in simple routing resp. covering-based routing with uniformly distributed one-dimensional values (cf. Equation 4.35) requires several sophisticated transformations to solve it analytically: 


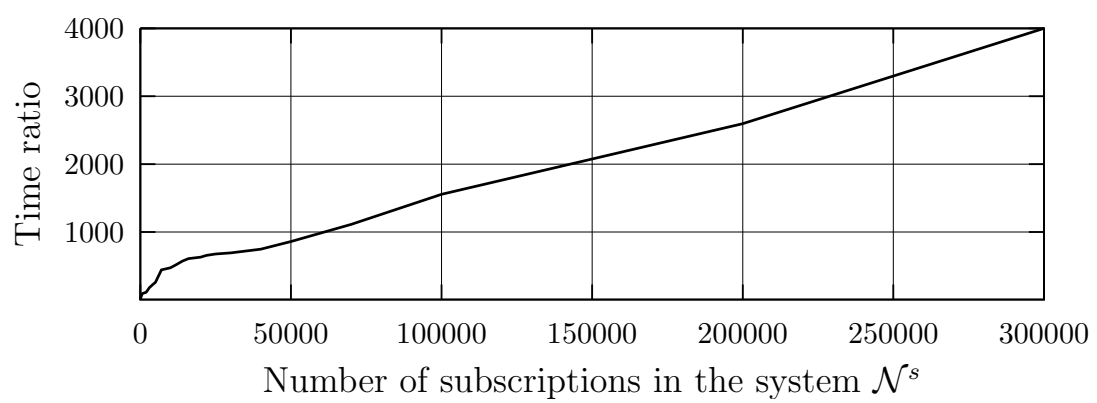

Figure 5.5: Runtime: simulation vs. analysis

$$
\begin{aligned}
p^{\text {match }}(k) & =\int_{0}^{1} 1-(1-2 \cdot x(1-x))^{k} \mathrm{~d} x \\
& =1-\sum_{l=0}^{k}\left(\begin{array}{l}
k \\
l
\end{array}\right)(-2)^{l} \int_{0}^{1} x^{l}(1-x)^{l} \mathrm{~d} x \\
& =1-\sum_{l=0}^{k}\left(\begin{array}{l}
k \\
l
\end{array}\right)(-2)^{l} \frac{(l !)^{2}}{(2 l+1) !} \\
& =1-\sum_{l=0}^{k}(-2)^{l} \frac{k !}{(k-l) !} \frac{l !}{(2 l+1) !}
\end{aligned}
$$

Although the derived result is analytical, it is not easy to calculate. Similar to the latter section, large numeric values in enumerator and denumerator may appear for large values of $k$ that could lead to imprecise results. Another potential way to calculate $p^{\text {match }}(k)$ is to see that the results contain the so called Generalized Hypergeometric Function ${ }_{2} F_{1}\left(a_{1}, a_{2} ; b_{1} ; x\right)$ which is a generalization of a Geometric series defined by 4 parameters. Thus, it holds $p^{\text {match }}(k)=1-{ }_{2} F_{1}(1,3 / 2+k ; 3 / 2 ;-1)$. This of course requires a lookup table to realize a gain in calculation speed. The problem of analytically solving integrals is that each integral has to be solved individually requiring lots of effort. Furthermore, it is not clear if the results really simplify the calculation. Therefore, we decided to solve each integral numerically. Since the integrands are not hard, most basic numerical integration, like rectangle or trapezoidal rule, are appropriate to use. To avoid a special treatment of improper integrals, we assume that each attribute dimension is limited by a fixed finite value which is an assumption that is met by all natural and technical systems.

\subsubsection{Efficiency}

Bringing all mentioned approaches together, we were able to efficiently calculate the stochastic model. Contrary to a discrete event simulation, our analytical approach is especially efficient for higher subscription birth and publication rates making such scalability evaluations very cheap. This is due to the fact that the approach does not need to deal with each message separately and its computational time does not depend on the message load. Figure 5.5 shows the relative 
execution times of the analysis compared to the runtime of a corresponding discrete event simulation in relation to the number of subscriptions. The results were determined for a 127 brokers topology (cf. Section 4.5) on a standard PC. A ratio of $x$ means that the simulation require $x$ times more time. One can see an approximately linear increase of the ratio. That means that the gain of using the system model increases if large-scale publish/subscribe systems are considered. But even for small numbers of subscriptions the analysis is always better than the discrete event simulation. The figure may vary if other parameters of the system change, such as number of brokers, or if another system architecture is used for simulation resp. model solving. However, the general qualitative behavior is the same which strongly encourages the advantages of having a publish/subscribe system model.

\subsection{Optimized Configurations}

In the previous section, we laid the foundation to efficiently calculate the complete model for one set of inputs. As already described in Chapter 3, designers are interested in optimized configurations for given boundary conditions. Thus, we explain how the model can be included into a scheme to determine optimal publish/subscribe infrastructures. To stress the complexity to get an optimized configuration we estimate the size of the parameter space.

\subsubsection{Optimizing Publish/Subscribe Infrastructures}

The first step of an optimization is to define the optimization goal. This is usually done by the designer. The developed system model (cf. Chapter 4) provides a lot of outputs for this purpose. These performance measures can either be directly used as the goal function of the optimization or they are part of a constraint that restricts possible solutions. Following, we provide exemplary optimization goals which are based on the optimization types provided in Section 3.3:

Minimal Message Delay. Based on the maximization principle: From all possible topologies $T$ determine the one with the lowest message delay (highest QoS) while not exceeding a maximum number of brokers in the topology $T_{\max }$. Formally, we get

$$
\min _{T} \Delta^{n}(T)+\Delta^{s}(T):|T| \leq T_{\max }
$$

Minimal Topology. Determine the minimal topology that guarantees that all brokers of the topology are not overloaded. Thus,

$$
\min _{T}|T|: \forall B \in T: U(B) \leq 90 \%
$$

This approach is based on the minimization principle from Section 3.3. Depending on the use case, many different definitions of the goal function and its constraints are possible. This way the designer may map all requirements to a formal criterion. 


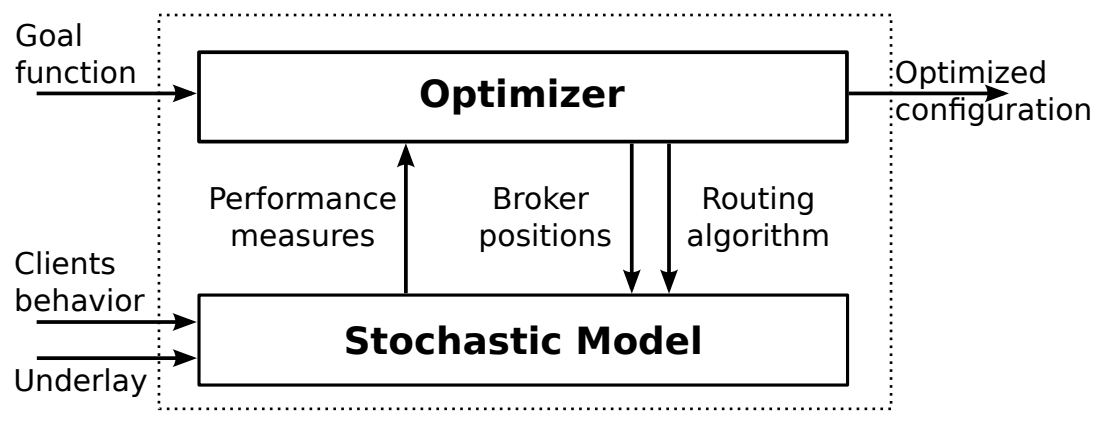

Figure 5.6: Overview on capacity planning

With this goal function, we go into the optimization scheme in Figure 5.6. It shows the general scheme of a capacity planning module and the information flow between the components, the optimizer and the analytical model. One can easily recognize inputs and outputs of the analytical model that were derived in Section 4. Since the environmental parameters, namely client behavior and underlay network, are also inputs for the capacity planning module, these parameters have to be known a priori or must be estimated by the designer. Especially, before a first setup of a system the client behavior can only be estimated, but at redesign phase measured values of the "real" behavior can be used to improve the results of the optimization. The free parameters, namely the overlay topology and the routing algorithm are optimization variables that are systematically manipulated by the optimizer to find a configuration according to the optimization goal. The output of the capacity planning module is an optimized configuration which consist of a list of brokers with their mapping to routers and the routing algorithm that has to be applied in the network. The resulting configuration should be used by the system designer to put the publish/subscribe system into production. If it is a redesign, then the operator must switch the system from the "old" suboptimal configuration to the new one.

Since the search-space of the optimization problem is discrete and grows very fast (cf. next subsection), heuristic optimization algorithms, like genetic algorithms [34] or simulated-annealing [50] are appropriate to use. Heuristics reduce the execution time significantly with only a slightly degradation in quality of the optimum. In this thesis, we skip the procedure of finding optimal optimization algorithms and its parameters. Instead, we focus on the research question of the optimization complexity.

\subsubsection{Optimization Complexity}

In this section we investigate the optimization complexity which is the number of possible configurations for a publish/subscribe system embedded into a physical underlay consisting of $n$ hosts. This complexity $C(n)$ is a hint on the heaviness of the optimization task, especially for large infrastructures. Starting point for the calculation is the number of hosts, where brokers may be placed in the underlay. To place a fixed number of $i$ brokers within this underlay there are $\left(\begin{array}{c}n \\ i\end{array}\right)$ possibilities, assuming that each host hosts at most one broker. Each set of $i$ 
brokers have to be interconnected with an acyclic overlay with $i-1$ overlay links. According to Cayley's formula ${ }^{1}$ there exist $i^{i-2}$ distinct overlays for $i$ brokers. Beside the overlay configuration, there exists a number of possible routing configurations. So far we had several standard algorithms. Later, in the chapter about adaptive routing (Section 6.3.4) we show that there are much more possible routing algorithms in publish/subscribe. Then, it is shown that the number of possible routing configurations depends on the topology. The range is between the two extremes, a line consisting of $i$ brokers with $C^{-}(i)=i^{2}$ routing configurations and a star consisting of a center and $n-1$ surrounding brokers with $C^{\star}(i)=2^{i}+i-2$ routing configurations. The last configuration parameter is the number of brokers which can be between 1 and $n$. Thus, after summing over 1 to $n$ brokers we get an estimation for the complexity $C(n)$ which is within the range $C_{\text {low }}(n) \leq C(n) \leq C^{u p}(n)$.

$$
\begin{aligned}
C_{\text {low }}(n) & =\sum_{i=1}^{n}\left(\begin{array}{l}
n \\
i
\end{array}\right) \cdot i^{i} \\
C^{u p}(n) & =\sum_{i=1}^{n}\left(\begin{array}{c}
n \\
i
\end{array}\right) \cdot i^{i-2} \cdot\left(2^{i}+i-2\right)
\end{aligned}
$$

Obviously, both limits grow rapidly when the number of physical nodes $(n)$ in the underlay is increased. To get a better idea about the exact behavior for a large number of nodes, we approximate these sums by applying the following mathematical conversions:

$$
\begin{aligned}
\frac{C_{\text {low }}(n)}{n^{n}} & =\lim _{n \rightarrow \infty} \frac{1}{n^{n}} \sum_{i=1}^{n}\left(\begin{array}{c}
n \\
i
\end{array}\right) \cdot i^{i} \\
& =\lim _{n \rightarrow \infty} \frac{1}{n^{n}} \sum_{i=0}^{n-1}\left(\begin{array}{c}
n \\
i
\end{array}\right) \cdot(n-i)^{n-i} \\
& =\lim _{n \rightarrow \infty} \sum_{i=0}^{n-1} \frac{1}{n^{i}}\left(\begin{array}{c}
n \\
i
\end{array}\right) \cdot\left(\frac{n-i}{n}\right)^{n-i} \\
& =\sum_{i=0}^{\infty} \lim _{n \rightarrow \infty}\left[\frac{1}{n^{i}}\left(\begin{array}{c}
n \\
i
\end{array}\right)\right] \cdot \lim _{n \rightarrow \infty}\left(1-\frac{i}{n}\right)^{n-i} \\
& =\sum_{i=0}^{\infty} \frac{1}{i !} e^{-i}=e^{\frac{1}{e}} \\
\frac{C^{u p}(n)}{n^{n-2} \cdot\left(2^{n}+n\right)} & =\lim _{n \rightarrow \infty} \frac{1}{n^{n-2} \cdot\left(2^{n}+n\right)} \sum_{i=1}^{n}\left(\begin{array}{c}
n \\
i
\end{array}\right) \cdot i^{i-2} \cdot\left(2^{i}+i-2\right) \\
& =\sum_{i=0}^{\infty} \lim _{n \rightarrow \infty}\left[\frac{1}{n^{i}}\left(\begin{array}{c}
n \\
i
\end{array}\right)\right] \cdot \lim _{n \rightarrow \infty}\left(1-\frac{i}{n}\right)^{n-i-2} \cdot 2^{-i} \\
& =\sum_{i=0}^{\infty} \frac{(2 e)^{-i}}{i !}=e^{\frac{1}{2 e}}
\end{aligned}
$$

\footnotetext{
${ }^{1}$ Cayley's formula can be found in any book about graph theory.
} 


\begin{tabular}{|c||c|c|c|c|c|c|c|c|c|}
\hline$n$ & 1 & 2 & 3 & $\ldots$ & 10 & $\ldots$ & 100 & $\ldots$ & 1000 \\
\hline \hline$C_{\text {low }}$ & 1 & 6 & 42 & $\ldots$ & $1.47 \cdot 10^{10}$ & $\ldots$ & $1.45 \cdot 10^{200}$ & $\ldots$ & $1.44 \cdot 10^{3000}$ \\
\hline$C^{\text {up }}$ & 1 & 6 & 42 & $\ldots$ & $1.31 \cdot 10^{11}$ & $\ldots$ & $1.53 \cdot 10^{226}$ & $\ldots$ & $1.29 \cdot 10^{3295}$ \\
\hline
\end{tabular}

Table 5.1: Complexity of optimization

That means that the sums can be approximated by $C_{\text {low }}(n) \approx 1.444 \cdot n^{n}$ resp. $C^{u p}(n) \approx 1.202 \cdot n^{n-2} \cdot\left(2^{n}+n\right)$. To give the reader an idea about the growing search space of the optimization, we added Table 5.1. The bounds $C_{\text {low }}(n)$ and $C^{u p}(n)$ are equal for $n=\{1,2,3\}$ since line and star do not differ for these numbers of brokers. For large $n$ the number of configurations rapidly increases implying that only an optimization algorithm can handle it.

\subsection{Case Study}

To validate the approach and demonstrate its effectiveness in a realistic scenario, we now present a case study of a representative publish/subscribe system. We consider a scenario in which a company that operates world-wide needs to exchange data (e.g., stock quotes, trade orders, and business news) with its customers and field staff in an efficient and timely manner. The company's activities are divided into different areas (e.g., countries), where each area runs some regional offices that may host publish/subscribe brokers. In order to plan the capacity of the system, the company needs to know the optimal number of brokers and their placement in the network, as well as the resulting utilization of links and brokers, and the expected system performance in terms of end-to-end notification and subscription delays.

A scenario is defined by a static and a dynamic part. The static part represents parameters that are usually fixed in reality and includes the network topology as well as the positions of clients and company offices that may host brokers. The dynamic part is used to describe concrete use cases and workloads characterized by message traffic and the locality of subscriptions and notifications.

The physical topology is created with 5 domains (representing the areas) and about 1000 routers (possible locations of clients) using the BRITE [64] topology generator. To derive an Internet-like topology, the transit-stub model is applied. The parameters for the generation are set according to Table 5.2 and are based on Jaeger [43].

The capacity of the physical links varies between 30,000 and 60,000 messages per second, and the capacity of physical routers is set to 250,000 messages per second. Within this topology, 100 clients (representing customers and field staff that produce and consume notifications) are positioned by attaching them to randomly chosen routers in the physical topology. Next, two special locations per domain are selected that represent the company's regional offices that may be used to host a broker. For a given broker placement using some or all of these locations, each broker has a capacity of 1400 messages per second and is connected to the other brokers by a minimum spanning tree based on the distance in the physical topology measured in hops. The clients are connected to the nearest broker. 


\begin{tabular}{|l|l|}
\hline Topology Type & 2 Level: TOP-DOWN \\
\hline HS & 1000 \\
\hline N & 100 \\
\hline LS & 100 \\
\hline Model & Waxman \\
\hline Node Placement & Heavy Tailed \\
\hline alpha & 0.15 \\
\hline Growth Type & Incremental \\
\hline beta & 0.2 \\
\hline Pref. Conn. & None \\
\hline m & 2 \\
\hline \multicolumn{2}{|c|}{ Route } \\
\hline HS & 1000 \\
\hline N & 100 \\
\hline LS & 100 \\
\hline Model & Waxman \\
\hline Node Placement & Heavy Tailed \\
\hline alpha & 0.15 \\
\hline Growth Type & Incremental \\
\hline beta & 0.2 \\
\hline Pref. Conn. & None \\
\hline m & 2 \\
\hline \multicolumn{2}{|c|}{ Top Down } \\
\hline Edge Connection Model & Random \\
\hline Inter BW Distr. & Heavy Tailed \\
\hline Max BW & 1024 \\
\hline Min BW & 10 \\
\hline Intra BW Distr. & Heavy Tailed \\
\hline Max BW & 1024 \\
\hline Min BW & 10 \\
\hline & \\
\hline & \\
\hline
\end{tabular}

Table 5.2: Parameters used for topology generation with BRITE 


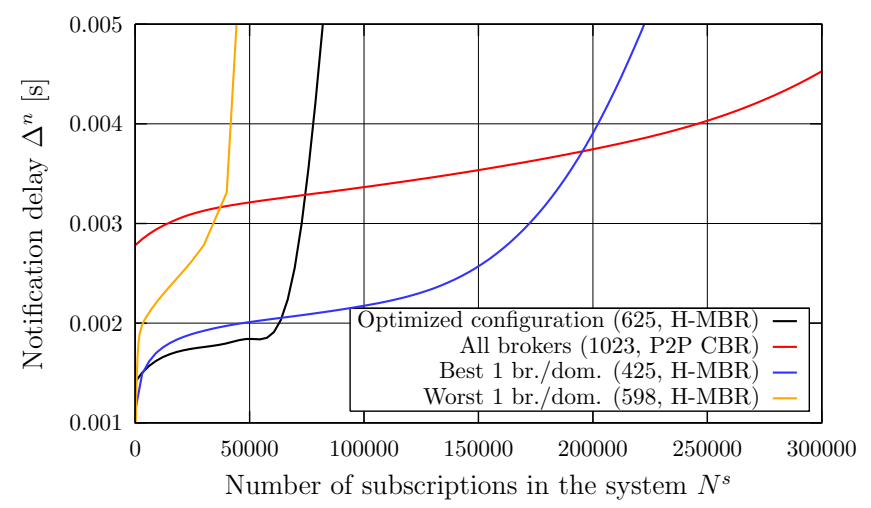

Figure 5.7: Notification delay for different configurations

On top of this static setting, a concrete scenario is described by a number of active subscriptions $N^{s}$ in the system with an exponentially distributed expected lifetime of $60 \mathrm{~s}$, a publication rate $\omega$ and a locality measure $L$ described below. For a given scenario, these parameters have to be known either by a good estimation or by data from a real scenario. The service times for processing messages at the brokers are equally distributed and have an exponential distribution independent of the message type. Each subscription and notification is assigned to one of 500 different filter classes. Regarding locality, each class has a randomly chosen hot spot domain in which a fraction $L$ of its notifications are produced and a fraction $L$ of its subscriptions originate from clients within that domain. The remaining subscriptions/notifications are uniformly distributed among the other four domains.

Capacity Planning. Given such a scenario description defined by a physical network topology, the possible broker positions, and a workload specification, capacity planning aims at answering the question where to best place how many brokers using which routing algorithm in order to minimize the average notification delay. Regarding the company example, there are ten possible broker positions (two in each of the five domains) leading to $2^{10}-1=1023$ different broker placements for which each of the seven individual routing algorithms has to be investigated. Thus, even this small-sized example finally leads to 7161 cases to be considered for just a single workload specification. Since in our case study the resources are limited but the performance is subject of the optimization we follow the maximization principle according to 5.4.

Up to now, discrete event simulation was the only viable approach to evaluate these scenarios and derive estimations for the traffic, message delays, and routing table sizes in advance. However, as simulation is costly in terms of computation time and memory requirements, one usually restricts the number of considered cases drastically by, e.g., adding further placement constraints and/or preselecting the routing algorithm. Thus, a manageable, conventional simulation strategy would be to place exactly one broker per domain (maybe evaluating all 32 permutations) and to compare the obtained results to a complete placement with all ten brokers. 
Leveraging the stochastic analysis derived above, however, we are able to cover a much larger parameter space. In fact, it becomes possible to solve this optimization problem by conducting an extensive search with justifiable resources in terms of computing power and time. For the company scenario with 50,000 active subscriptions and a locality of 0.8 , we determined the optimal broker placement and best routing configuration within minutes. The results are shown in Figure 5.7. The best solution leads to an expected notification delay of $1.8 \mathrm{~ms}$, employs hierarchical merging-based routing and uses five brokers which are not equally distributed over the domains: one domain has two, another has none, while the others have one. In reality, this means that some company offices have a bad connectivity within their domain while offices in neighbor domains are better connected. In the restricted simulation setup described above, this case would not be evaluated at all. Furthermore, if considering all 32 configurations with exactly one broker per domain, the results vary between $2 \mathrm{~ms}$ and $7 \mathrm{~ms}$ for the best (topology 425) and the worst case (topology 598). Thus, getting a good candidate randomly is quite unlikely.

Usually, parameters such as load and locality are estimated based on previous experience or expectations and are therefore subject to errors. This leads to systems facing workloads beyond the workload intensities for which they have been optimized. Therefore, it is necessary to investigate how scalable the derived configurations are. To illustrate this problem, in Figure 5.7, we varied the load from zero to 300,000 active subscriptions to study the impact on the different configurations including the optimal one. Obviously, the optimal configuration does not scale well and saturates for higher loads. On the other hand, configuration 425 is less sensitive for an increasing number of subscriptions and should be preferred although the delay for the optimization point is marginally higher. However, the best scalability is provided by a complete 10 broker topology, but with a significantly higher average delay. This example shows the need for a comprehensive system analysis that is hard to achieve with simulation-based approaches.

Advanced Evaluations. Beside capacity planning for concrete workload scenarios, the approach allows to gain further insights into the behavior of the publish/subscribe system without expensive simulation runs. In the considered setting, we were able to make several important observations described in the following.

First, we considered the selection of an optimal routing algorithm for a given subscription load. We derived the optimal (with respect to minimizing the maximum expected notification delay) routing algorithm for each of the 1023 broker placements, four different localities $(0.2,0.4,0.6$ and 0.8$)$ and a subscription load varying from 10 to 700,000 subscriptions in the system. Figure 5.7 shows the distribution of the four routing algorithms hierarchical covering-based (H-CBR), hierarchical merging-based (H-MBR), peer-to-peer covering-based (P2P-CBR) and peer-to-peer merging-based (P2P-MBR) in a way that the numbers represent the fraction of cases where the appropriate algorithm is optimal. It is easy to see that peer-to-peer merging-based routing is optimal for low load, hierarchical covering-based routing for medium load and hierarchical merging-based routing for high load. For the given setting, this insight might help to optimize the system behavior in case of only vague knowledge on expected load. 


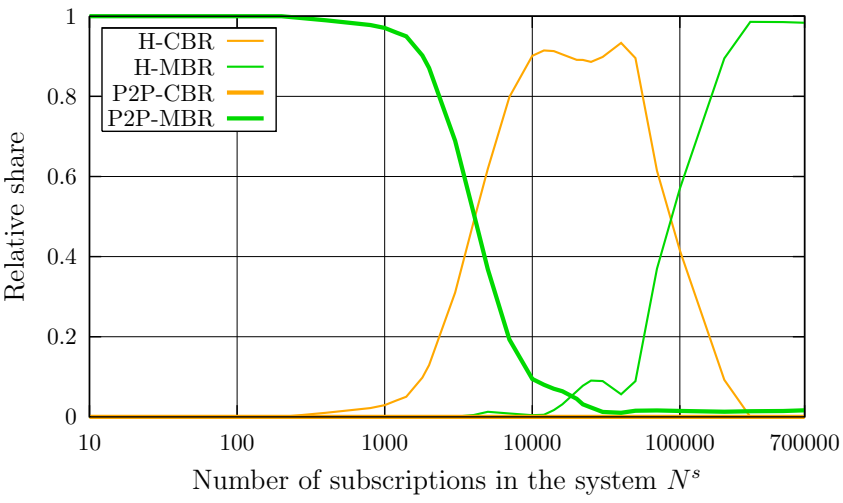

Figure 5.8: Optimal routing algorithms and load

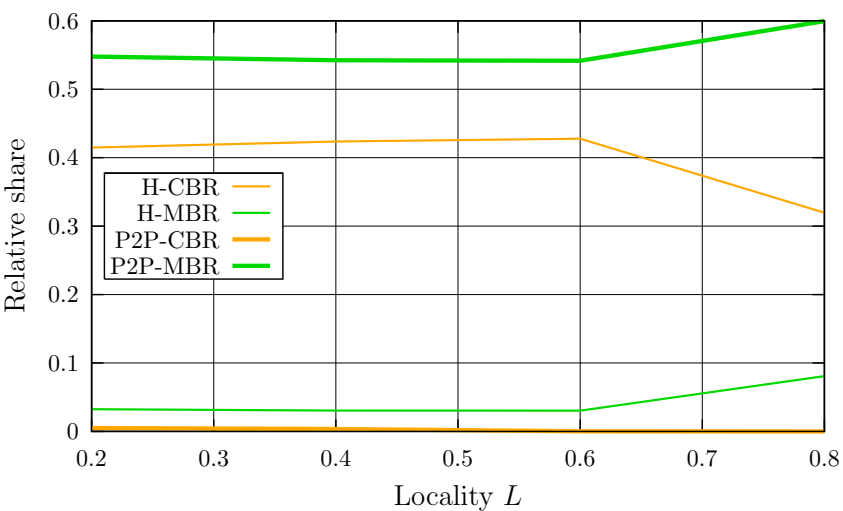

Figure 5.9: Optimal routing algorithms and locality 
Second, we analyzed the impact of locality. Figure 5.9 shows the distribution of optimal routing algorithms for different localities based on the experiment described above (with load and locality exchanged so that each data point represents results from evaluating 1023 broker placements with varying load). Here, we see that both types of merging-based routing profit from high locality while for hierarchical covering-based routing the opposite effect can be seen. Furthermore, in the setting locality seems to influence the selection of the optimal routing algorithm much less than it is influenced by the number of subscriptions.

The described insights are just two examples of how the proposed stochastic modeling and the capacity planning approach allows to analyze the impact of design decisions on the system performance without the need for expensive and time consuming simulations. With the help of optimizers that usually determine only a subset of all possible configurations even large-scale systems can be optimized.

\subsection{Discussion}

The goal of this section was to show how the stochastic system model of Chapter 4 can be used for provisioning a system. Therefore, we first explained how the model can be calculated efficiently. We introduced a workflow for the calculation and we solved numerical challenges in several formulas. With these results we were able to derive a capacity planning module which can be used in the (re)design phase of the life cycle of a publish/subscribe as introduced in Section 3.4. Furthermore, we discussed the complexity of such an optimization. We closed this chapter with a case study of a representative publish/subscribe system. Although, the case study is only based on simulation data and does not use sophisticated optimization algorithms, we were able to show the applicability of the approach.

As already mentioned in the discussion of the previous chapter (cf. Section 4.7) the lack of real data undermines a deeper evaluation. If these data are once made available the model can be adapted based on the existing extensions provided in Section 4.6. Furthermore, parameters for the physical network such as link capacities must be identified. If this is not sufficient model extensions are required. Finally, an important step is to investigate which optimization algorithm fits best to the problem domain. Before going to genetic algorithms or simulated-annealing, standard methods should be investigated because the optimization problem may behave friendlier than expected. In order, to increase the optimization time the usage of GPUs would be interesting. When integrating all these things into one capacity planning tool, the designer is able to provisioning large-scale publish/subscribe systems. Thereby, any requirements definitions can be fulfilled that consider both the QoS for clients and the costs for operators. 


\section{Chapter 6}

\section{Adaptive Routing}

\section{Contents}

6.1 Introduction ................... 118

6.2 Related Work . . . . . . . . . . . . . 119

6.3 Hybrid Routing Algorithms . . . . . . . . . 120

6.3.1 Rendezvous Broker and Correctness Criterion . . . . . . . . 121

6.3.2 Routing Configurations . . . . . . . . . . . . . . . . . . . . . . 124

6.3.3 Routing Reconfigurations . . . . . . . . . . . . . . . . . . . . . . 125

6.3.4 Structure of Routing (Re)configurations . . . . . . . . . 127

6.4 Self-optimizing Algorithm . . . . . . . . . 130

6.4.1 Optimization Criterion . . . . . . . . . . . . . . . 130

6.4 .2 Algorithm Details . . . . . . . . . . . . . . . . . . . . . . . . . . . . . . . . .

6.5 Evaluation . . . . . . . . . . . . . . 140

6.5.1 Step Response . . . . . . . . . . . . . . . . . . 140

6.5 .2 Dynamic Situation . . . . . . . . . . . . . . . . 143

6.6 Generalizations and Extensions . . . . . . . . . 144

6.6.1 Adaptive Hierarchical Routing . . . . . . . . . . . . . 145

6.6.2 Advanced Routing Algorithms and Advertisements . . . . . 146

6.6.3 Fault-tolerant Adaptive Routing . . . . . . . . . . . . . . . 147

6.6.4 Fault-tolerant Adaptive Hierarchical Routing. . . . . . . . . 148

6.6.5 Composition of Self-optimizing Algorithms . . . . . . . . . 148

6.7 Discussion ...................... 151 


\subsection{Introduction}

In the previous chapter, we introduced an offline capacity planning strategy that can be used for determining an optimized configuration for the overlay network based on measured parameters. Since the behavior of the clients and the capacity of brokers, routers and links can vary over time, the network performance and costs can change, and e.g., become worse. This may lead to the violation of expected performance measures what further could lead to reduced acceptance of the system. Thus, to avoid a system performance degradation and cost increase, it is necessary to react at runtime, too.

From the input parameter identification in Section 4.3 it is known that there are two parameters which can be set freely, the overlay topology and the routing algorithm. While in capacity planning these parameters were manipulated as inputs of the introduced stochastic model we are now going to adapt them at runtime. Therefore, the publish/subscribe middleware must provide new "knobs", i.e., the possibility to change these parameters which includes mechanisms that avoid message loss and duplication. This stems from the correctness guarantee a publish/subscribe system must provide to clients as we introduced it in Definition 2.4.2.

In this chapter the focus is on adapting the routing. This approach introduces a new type of self-optimizing algorithm that combines different standard routing algorithms within one overlay network. It is well-known that depending on the client's distribution and the dynamics of their interests and publishing behavior, different routing algorithms are better suited in different situations [65]. For example, flooding can be beneficial in situations where clients are widespread and are interested in almost all published notifications. Contrarily, in situations with a high locality filtering-based algorithms like simple routing or covering-based routing should be used. The evaluations in Sections 4.5 and 5.5 confirmed this observation. However, current publish/subscribe middleware implementations usually enforce the usage of a single, statically configured algorithm employed system-wide. Obviously, this leads to suboptimal results as distributions, interests, and publications may change over time and may also substantially differ in distinct parts of the network. Furthermore, it significantly hinders the application of publish/subscribe systems in large-scale and dynamic environments.

To overcome these limitations, we introduce the new class of hybrid routing algorithms in Section 6.3 (after presenting the related work in Section 6.2) that allows the usage of more than one routing algorithm within an overlay network. Based on a seamless reconfiguration between different instances of hybrid routing algorithms, it is possible to optimize the systems performance by reducing the transmission and processing costs of messages. This idea is presented in Section 6.4, where we also derive a self-optimizing algorithm, called adaptive routing, and point out its major properties. In Section 6.5 we evaluate this approach by focusing on its behavior in dynamic situations. Following in Section 6.6, we qualitatively discuss important generalizations and extension of adaptive routing. Our special focus there is on the composition of adaptive routing and other self-optimizing algorithms. We end up this chapter with a discussion in Section 6.7. 


\subsection{Related Work}

The first, basic steps to optimize the routing in distributed publish/subscribe systems were the introduction of subscription forwarding which avoid unnecessary deliveries of notifications and the introduction of advertisements to avoid unnecessary deliveries of subscriptions [21, 22]. Further developments led to hierarchical routing as well as rendezvous point based approaches [80, 85]. All these approaches which were described in Section 2.4 have two drawbacks. First, they use a single routing scheme within one network and thus cannot deal with locally different behavior of clients. Second, all these algorithms are not adaptive. Instead the routing configuration is set at design or deployment time.

Using several routing algorithms in a single publish/subscribe overlay was first suggested by Carzaniga [19]. He proposed to have clusters using hierarchical routing that are connected by an acyclic peer-to-peer protocol. He assumed that the structure of such a hybrid topology is derived manually from requirements in companies by the system administrators. This approach allows to better fit the routing algorithm on local differences in the system than with using only one equal routing algorithm in the whole system. Nevertheless, this approach is neither adaptive to dynamic changes nor it utilizes the entire possibilities of routing algorithm combinations.

Another related approach of Bickson et al. [11] intelligently combines IP multicast and point-to-point unicast for the efficient dissemination of the information. This leads to the so called channelization problem which is NP-Hard. The authors solve this problem with the help of heuristics in a centralized way. Furthermore, the applicability of the approach is restricted due to the focus on channel-based publish/subscribe systems.

To react on changes in the environment, several approaches have been introduced to render the routing of publish/subscribe systems self-optimizing. Bittner and Hinze [12], for example, introduced subscription pruning. It reduces the matching costs by replacing complex subscription predicates by more general predicates, which are simpler to evaluate. To decide which predicates should be pruned, each broker determines a measure called selectivity degradation. This algorithm is focused on matching costs and the related memory consumption to store subscriptions. This potentially leads to increased costs for forwarding notifications since more notifications may be forwarded in which no client is interested in. Hence, the authors assume to have only marginal false positive notifications in the system.

An approach also focusing on the reduction of matching costs is the one of Jerzak and Fetzer [47]. They add a Bloom filter encoded prefix to an event when it enters the system and is matched for the first time. This prefix is then used to simplify further matchings within the overlay network. Generally, this approach is not adaptive and the authors do not answer the question if filtering may not be necessary in some cases.

In opposite to what we do, some authors (Li et al. [59], Briones et al. [15]) assume to have cyclic overlay networks, where several paths between two clients exist. Therefore, they focus on how to find optimal routes for notifications. This optimization is based on non-standard routing algorithms and targets an orthogonal dimension to optimize the routing. While our approach in principle pursuits the same objective, it dynamically adapts the applied routing algorithms to achieve cost reductions instead of modifying subscriptions or chang- 


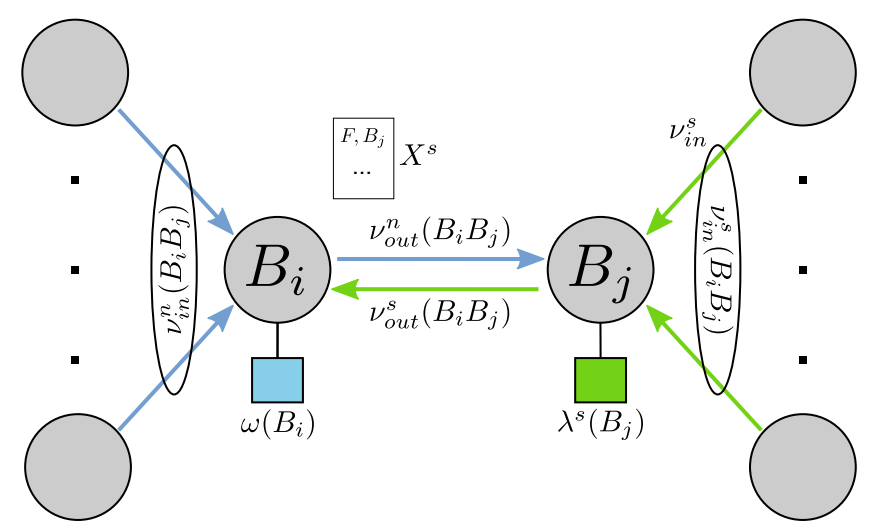

Figure 6.1: Link configuration

ing the overlay topology. Thus, our approach can be seen as orthogonal concept that may be combined with the others.

\subsection{Hybrid Routing Algorithms}

The foundation of adaptive routing is a new class of routing schemes which we call hybrid routing algorithms. We generally define that a hybrid routing algorithm combines different standard algorithms within one overlay network. Each instance of hybrid algorithm is described by a routing configuration $R$ that contains the routing algorithm used on each directed overlay link, i.e., there are two configurations for both directions on one link. A single link configuration connecting two arbitrary brokers $B_{i}$ and $B_{j}$ is characterized by $r_{i j}$. The value of $r_{i j}$ can take all types of basic algorithms (cf. Section 6.6.2). Without limiting the generality we now assume that $r_{i j}$ is either FL for flooding or SR for simple routing. Practically, both related brokers $B_{i}$ and $B_{j}$ store $r_{i j}$ in a consistent way. Therefore, each broker has a data structure for its routing configurations with a size which is twice the number of brokers overlay links. When for all overlay links in an overlay network $r_{i j}=F L$ or $r_{i j}=S R$ is selected, we call this routing configuration homogeneous resp. homogeneous routing. Homogeneous flooding and homogeneous simple routing are equal to the original flooding and simple routing as they were defined in Section 2.4. Later we will see that hierarchical routing which we also introduced earlier is also a subset of hybrid routing algorithms.

The central idea of hybrid routing algorithms is to transfer the global nature of a certain routing algorithm to a single overlay link $B_{i} B_{j}$. Figure 6.1 shows an arbitrary link configuration $r_{i j}$ between two brokers. For such a link configuration the direction plays an important role. The direction is the same as the direction of the notification flow from $B_{i}$ to $B_{j}$ measured by $\nu_{\text {out }}^{n}\left(B_{i} B_{j}\right)$. This flow is a subflow of the flow of all incoming notifications from brokers in $\mathcal{P}\left(B_{i} B_{j}\right)$ and the local clients and is measured by $\nu_{i n}^{n}\left(B_{i} B_{j}\right)$. The filtering of $B_{i}$ is based on its routing table entries with destination $B_{j}$. This filter list has the size $X^{s}\left(B_{i} B_{j}\right)$. These entries are build up by a reverse flow of subscriptions 
measured by $\nu_{\text {out }}^{s}\left(B_{i} B_{j}\right)$. This flow is a subflow of $\nu_{i n}^{s}\left(B_{i} B_{j}\right)$ which contains all incoming messages received by $B_{j}$ that may be potentially forwarded to $B_{i}$. This general description holds for all routing algorithms.

In case of flooding $\left(r_{i j}=F L\right)$, no subscriptions are forwarded from $B_{j}$ to $B_{i}$ and notifications from the set of predecessing links $\mathcal{P}\left(B_{i} B_{j}\right)$ are flooded to $B_{j}$. The routing table of $B_{i}$ simply contains a True-Filter with destination $B_{j}$. Formally, the following equations hold: $\nu_{i n}^{n}\left(B_{i} B_{j}\right)=\nu_{\text {out }}^{n}\left(B_{i} B_{j}\right), \nu_{\text {out }}^{s}\left(B_{i} B_{j}\right)=0$ and $X^{s}\left(B_{i} B_{j}\right)=1$. In the case of simple routing $\left(r_{i j}=S R\right)$, broker $B_{j}$ sends all incoming subscriptions from the set of successing links $\mathcal{S}\left(B_{i} B_{j}\right)$ and from local subscriptions to $B_{i}$ while $B_{i}$ filters incoming notifications according to its routing table before forwarding them to $B_{j}$. This leads to the following equation: $\nu_{\text {in }}^{s}\left(B_{i} B_{j}\right)=\nu_{\text {out }}^{s}\left(B_{i} B_{j}\right)$. About $\nu_{\text {out }}^{n}\left(B_{i} B_{j}\right)$ and $X^{s}\left(B_{i} B_{j}\right)$ no simple expressions are possible since these measures depend on the situation in the network. For other routing algorithms, a similar argumentation can be done.

What is important to realize is that we only argue in terms of brokers $B_{i}$ and $B_{j}$ when we describe the algorithm. Thus, we moved the focus from the global view on a routing algorithm as we made it in Section 2.4 to an overlay link related view. Thus, we claim, that a routing algorithm is not defined by its global but by its local behavior which is close to the definition of a distributed system. Only in some special cases of homogeneous or hierarchical routing we are able to see a consistent global behavior. Thus, the routing algorithm on an overlay link $B_{i} B_{j}$ is described by the notification filtering behavior of $B_{i}$ and the subscription forwarding behavior of $B_{j}$.

Based on this view, we can configure each directed overlay link with its individual routing configuration $r_{i j}$ forming one instance of a hybrid routing algorithm. In the following, we often call one such instance a routing configuration. In Figure 6.2, we show two exemplary routing configurations of an overlay network consisting of six brokers and ten directed overlay links. To indicate the routing configurations we marked links that use flooding with solid lines, while links with simple routing are dashed. Both routing configurations differ in one link configuration $r_{23}$. Despite this small difference, both routing configuration differ in one fact - their validity. While the left configuration is valid, the right figure shows an invalid routing algorithm. This is, because so far, we only formally composed different algorithms within one overlay. To guarantee the delivery of notifications as in Definition 2.4.2, we have to introduce a correctness criterion that limits the degree of freedom for the routing configurations. Therefore, in the following section, we derive a correctness criterion that must be met by all valid routing configurations.

\subsubsection{Rendezvous Broker and Correctness Criterion}

The correctness criterion for hybrid routing algorithms is a consequence of the correctness definition 2.4.2 for publish/subscribe systems. To derive this criterion, we consider the common ground for all routing algorithms, the rendezvous broker. A rendezvous broker $R_{i j}$ is a broker where the notifications (of the publishers) of a certain broker $B_{i}$ and the subscriptions (of the subscribers) of another broker $B_{j}$ meet. To work properly, each routing algorithm must guarantee that for all pairs of brokers the rendezvous broker is eventually reached by the subscriptions and the notifications. Subscription messages must leave state on the path to $R_{i j}$ which is done by establishing routing entries on the 


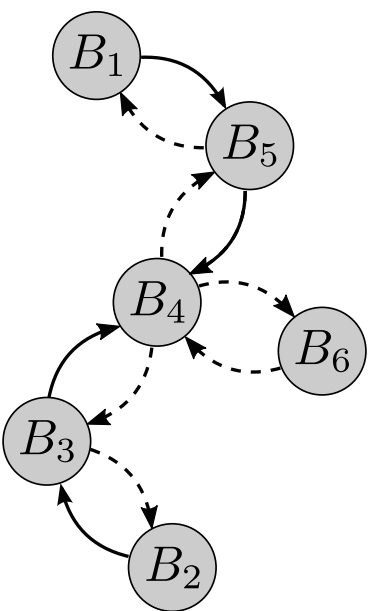

(a) Valid Configuration

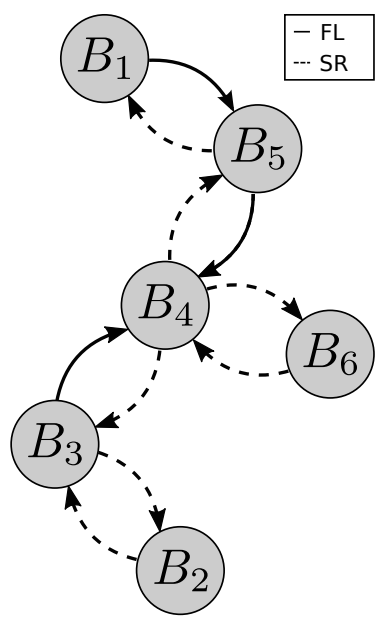

(b) Invalid Configuration

Figure 6.2: Routing configurations

visited brokers. This builds a reverse path for the delivery of the interesting notifications that eventually reach the rendezvous broker.

Recalling the standard algorithms from Section 2.4 we easily see that the rendezvous broker for flooding is $R_{i j}=B_{j}$. Here, subscriptions and notifications meet at the subscriber hosting broker. Hence the path to go for subscriptions is empty, no state information has to be stored in the system despite the local routing entries. In simple routing it holds that the rendezvous broker is the publisher hosting broker $\left(R_{i j}=B_{i}\right)$. This requires to establish a long reverse path and a lot of state information have to be stored in the system. Advanced routing algorithms, such as covering-based routing, reduce the amount of state information by an intelligent comparison of the current state (routing table) and incoming updates (subscriptions). Thereby, also the number of state updates is reduced. Although, in this case not all subscriptions reach the publishing broker, the expressed interest has been forwarded to it before. Therefore, it also holds $R_{i j}=B_{i}$. For hierarchical routing, the rendezvous broker $R_{i j}$ is that broker $B_{k}$ which is the head of the smallest subtree containing both brokers $B_{i}$ and $B_{j}$. Therefore, leaf brokers cannot be rendezvous brokers but all other brokers including the root broker $R$ can. In opposite to the general case, in hierarchical routing the rendezvous broker is symmetric, i.e., $R_{i j}=R_{j i}$.

In literature there are several approaches that explicitly or implicitly use rendezvous brokers. For example, in [85] a topic based publish/subscribe system uses a hash function to determine the rendezvous broker. In this case, $R_{i j}$ does not depend on the publisher or subscriber hosting broker but on the content resp. the topic of the subscription or notification. Furthermore, in [15] and [24] brokers are organized in a two- resp. multidimensional lattice. By disseminating notifications and subscriptions along different dimensions, a rendezvous broker is implicitly determined. It is the resulting intersection broker an this lattice.

Following this insight, we can derive that also a hybrid routing configuration must guarantee that for each pair of brokers a rendezvous broker is determined 


\begin{tabular}{|c||c|c|c|c|c|c|}
\hline Sub/Not & $B_{1}$ & $B_{2}$ & $B_{3}$ & $B_{4}$ & $B_{5}$ & $B_{6}$ \\
\hline \hline$B_{1}$ & $B_{1}$ & $B_{4}$ & $B_{4}$ & $B_{4}$ & $B_{5}$ & $B_{6}$ \\
\hline$B_{2}$ & $B_{5}$ & $B_{2}$ & $B_{3}$ & $B_{4}$ & $B_{5}$ & $B_{6}$ \\
\hline$B_{3}$ & $B_{5}$ & $B_{3}$ & $B_{3}$ & $B_{4}$ & $B_{5}$ & $B_{6}$ \\
\hline$B_{4}$ & $B_{5}$ & $B_{4}$ & $B_{4}$ & $B_{4}$ & $B_{5}$ & $B_{6}$ \\
\hline$B_{5}$ & $B_{5}$ & $B_{4}$ & $B_{4}$ & $B_{4}$ & $B_{5}$ & $B_{6}$ \\
\hline$B_{6}$ & $B_{5}$ & $B_{4}$ & $B_{4}$ & $B_{4}$ & $B_{5}$ & $B_{6}$ \\
\hline
\end{tabular}

Table 6.1: Rendezvous broker $R_{i j}$

to result in a correct publish/subscribe routing. If we recall the topology in Figure 6.2(a) with its valid routing configuration, we are able to build up Table 6.1 that contains rendezvous brokers $R_{i j}$ for any combination of brokers. It is important to note that the table is not necessarily symmetric, and that for the diagonal it holds $R_{i i}=B_{i}$. The invalid routing configuration in Figure 6.2(b) does not have a rendezvous broker for $R_{2 i}$ where $i \neq 2$. It is neither $B_{2}$ which does not have all subscriptions to filter nor $B_{4}$ which does not receive all notifications. When choosing any two brokers from an overlay with a valid routing configuration, the rendezvous broker lies on the connecting path separating it in a (possibly empty) flooding and a (possibly empty) simple routing zone. The rendezvous broker itself has an incoming FL link and an outgoing SR link. Please note that the order plays an important role. Following the direction of the links, SR must follow FL. This situation is depicted in Figure 6.3(a). The FL zone guarantees that all notifications reach the rendezvous broker, the SR zone guarantees that all routing information is available at the rendezvous broker. Any interrupt of both zones by another configuration is not allowed and would lead to notification message loss. This situation is shown in Figure 6.2(b), where the rendezvous broker for $B_{2} B_{4}$ is not defined. No broker out of $B_{2}, B_{3}$ and $B_{4}$ has all interest information and notifications. Therefore, on this path it cannot be guaranteed that notifications reach the interested subscribers.

With these examples in mind we can define valid routing configurations. If on one link $B_{i} B_{j}$ simple routing is configured it requires that all outgoing link in $\mathcal{S}\left(B_{i} B_{j}\right)$ are also using $\mathrm{SR}$. This is needed, because forwarding notifications from $B_{i}$ to $B_{j}$ requires to know the interests of the brokers in $\mathcal{S}\left(B_{i} B_{j}\right)$. Formally, we get:

$$
\forall B_{i} B_{j} \in T: r_{i j}=S R \rightarrow \forall B_{k} B_{l} \in \mathcal{S}\left(B_{i} B_{j}\right): r_{k l}=S R
$$

Colloquial we may say: "SR after SR". An equivalent definition is possible for flooding. Here "FL before FL" must hold to force predecessing links to not filter. Thus, we get:

$$
\forall B_{i} B_{j} \in T: r_{i j}=F L \rightarrow \forall B_{k} B_{l} \in \mathcal{P}\left(B_{i} B_{j}\right): r_{k l}=F L
$$

Both criteria are complementary meaning that checking one is sufficient to determine the validity of a hybrid routing algorithm. Thus, one only has to check either all flooding or all simple routing links. Both, criteria only consider direct neighbors. Recursively applied it creates the necessary delivery path for notifications between publishers and subscribers which results in a correct routing. 


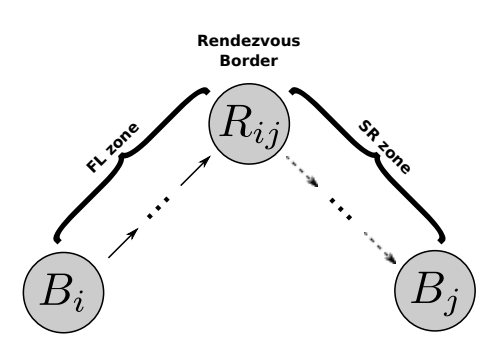

(a) Rendezvous broker $R_{i j}$

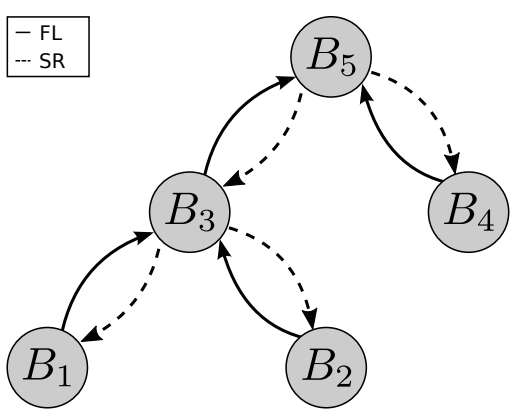

(b) Hierarchical routing

Figure 6.3: Rendezvous broker

\subsubsection{Routing Configurations}

As already mentioned, a routing configuration $R$ of a broker overlay is the set of the routing configurations $r_{i j}$ of all overlay links (for both directions). In case of homogeneous flooding or simple routing, all $r_{i j}$ are set to $F L$ and $S R$, respectively. Beside these special cases there is a big variety of valid configurations. Following, we analyze the special case of hierarchical routing and show that it is a valid routing configuration. Then, we show how the routing configuration is embedded into the stochastic system model introduced in Chapter 4. Doing so, we enable adaptive routing for the analysis. A discussion about the structure of valid routing configurations is given in Section 6.3.4.

\section{Hierarchical Routing}

In hierarchical routing (cf. Section 2.3), there is a dedicated root broker $R$ to which all notifications and subscriptions are flooded. Thus, the acyclic overlay converts to a rooted tree. On the way down the tree, notifications are filtered by active subscriptions. In contrast, subscription messages are not sent down the tree - they are sent upwards. Translated to hybrid routing algorithms, this means that downstream filtering algorithms, like simple routing, are configured, while upstream messages are flooded. In Figure 6.3(b) an exemplary setting of hierarchical routing is depicted with $B_{5}$ as the root broker. According to the previous description, all directed overlay links from the root to the leaves are dashed symbolizing simple routing, while the reverse links are solid symbolizing flooding. Thus, the hierarchical routing are a special instances of hybrid routing algorithms. Furthermore, hybrid routing generalizes the routing dimension of hierarchical routing as it has been introduced in Section 2.4. With the help of the correctness criteria 6.1 resp. 6.2 , the correctness can be easily shown.

\section{System Model Extension}

In Section 4.5.3, we have shown which changes have to be done to extend the stochastic system model for hierarchical routing. These changes referred to the forwarding probabilities $P^{n}\left(B_{i} B_{j}\right)$ and $P^{s}\left(B_{i} B_{j}\right)$ and the calculation of the number of expected remote routing entries $X^{s}\left(B_{i} B_{j}\right)$. In each case, we set the 
measure to 0 resp. 1 depending on the direction of the considered link, i.e., whether $B_{i} B_{j}$ is downstream or upstream relative to the root broker. What we implicitly did was to simply assign the formula for flooding with $P^{n}\left(B_{i} B_{j}\right)=1$ and $P^{s}\left(B_{i} B_{j}\right)=0$ and $X^{s}\left(B_{i} B_{j}\right)=0$ to upstream links. Thus, to extend the system model of Chapter 4 to support arbitrary hybrid routing algorithms we set $P^{n}\left(B_{i} B_{j}\right), \quad P^{s}\left(B_{i} B_{j}\right)$ and $X^{s}\left(B_{i} B_{j}\right)$ for all overlay links $B_{i} B_{j}$ depending on its individual routing configuration $r_{i j}$. These routing algorithm dependent formulas are given in Sections 4.4.6 and 4.4.7 resp. as compact overview with other formulas in Table 4.4. This way, adaptive routing is fully integrated into the stochastic system model without an additional derivation of formulas.

\subsubsection{Routing Reconfigurations}

In this section, we derive how to switch from one correct configuration to another. With this mechanism, an administrator or an algorithm for selfoptimization (introduced in Section 6.4) can adapt the routing scheme in the overlay network at runtime in a distributed manner.

Formally, we denote a reconfiguration of an overlay link $B_{i} B_{j}$ by $r_{i j}=\left[r_{c}, r_{t}\right]$ with $r_{c} \neq r_{t}$ and $r_{c}, r_{t} \in\{F L, S R\}$, where $r_{c}$ is the current configuration and $r_{t}$ the target configuration. Thus, we get two reasonable reconfigurations from FL to SR and vice versa. Such an intended reconfiguration $r_{i j}$ cannot be made in any order. Instead, each time it must be checked if before and after the reconfiguration the derived correctness criteria for the routing configuration is met. Furthermore, the reconfiguration process must not violate the correctness of the notification service. Following, we show for both possible reconfigurations which of the two involved brokers $B_{i}$ or $B_{j}$ is the broker that can decide if such a reconfiguration is possible. We call this broker the coordination broker. Furthermore, we explain the reconfiguration process.

Switching from Flooding to Simple Routing. A (valid) reconfiguration of an overlay link $B_{i} B_{j}$ from flooding to simple routing $r_{i j}=[F L, S R]$ must be controlled broker $B_{j}$ which is the coordination broker in this case. Only this broker can directly decide whether this reconfiguration is possible due to Equation 6.1. It just checks if (according to its internal routing configuration data structure) all links in $\mathcal{S}\left(B_{i} B_{j}\right)$ are configured to use $\mathrm{SR}$, so that $r_{i j}=S R$ is valid after the reconfiguration. If this is true, broker $B_{j}$ can start the reconfiguration process. In case that $B_{j}$ is a leaf broker, $\mathcal{S}\left(B_{i} B_{j}\right)$ is an empty set and, thus, $B_{j}$ can always switch its incoming link to SR. For now, we ignore that such a reconfiguration may not be reasonable because it could increase the overall traffic in the overlay. In Section 6.4, we provide an easy criterion when such a reconfiguration should be triggered. The process of the reconfiguration requires several steps to not provoke notification loss or duplicates and transferring the system from one valid routing configuration to the target configuration.

When broker $B_{j}$ has decided to process $r_{i j}=[F L, S R]$, it first forwards all routing table entries that point to brokers in $\mathcal{S}\left(B_{i} B_{j}\right)$. This can be implemented by a single bulk message or by sending all required routing table entries one by one. Broker $B_{i}$ processes these subscriptions in a normal way by adding them to its routing table. This way, $B_{i}$ gets all interests of $B_{j}$. This procedure implicitly requires that a broker is always ready to receive subscriptions even if the related overlay link is configured to use $F L$. During this phase, all 


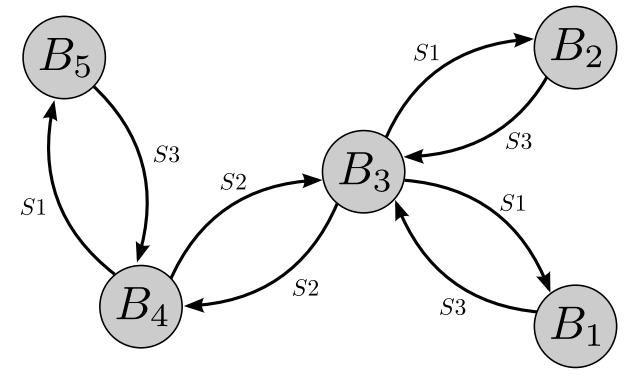

Figure 6.4: Reconfiguration from flooding to simple routing

subscriptions and unsubscriptions that are received by $B_{j}$ are buffered. After all routing table entries have been forwarded, $B_{j}$ sends a signal to $B_{i}$ to let it start filtering notifications on $B_{i} B_{j}$. We call this message StartFilteringNotifications or shorter $S F N$. When broker $B_{i}$ received this message it can be sure that its routing table is complete and that it represents the same interest as $B_{j}$ interests at the time when it decided to reconfigure. After the activation of the filtering, $B_{i}$ acknowledges the completion by sending an acknowledgement message $S F N A$ back to $B_{j}$. When $S F N A$ is received by $B_{j}$, it flushes its message buffer and starts to directly forward all subscriptions and unsubscriptions to $B_{i}$ that are received from other brokers. This completes the reconfiguration to simple routing.

With the described reconfiguration of a single link we are able to stepwise reconfigure the whole overlay network. Figure 6.4 shows an example process of a complete reconfiguration from homogeneous flooding to homogeneous simple routing. The figure depicts a broker overlay consisting of brokers $B_{1}, \ldots, B_{5}$ and their connecting overlay links. To illustrate the process of reconfiguration, we added a label to every link. The labels $S 1$ to $S 3$ express the causal relation between the link reconfigurations. $S 2$ directly depends on $S 1$, and $S 3$ directly depends on $S 2$. The first possible reconfigurations are, thus, $r_{3,1}=[F L, S R], r_{3,2}=[F L, S R]$ and $r_{4,5}=[F L, S R]$, because $B_{1}, B_{2}$, and $B_{5}$ are leaf brokers with an empty set $\mathcal{S}\left(B_{i} B_{j}\right)$. All other links have to apply flooding because the criterion of Equation 6.2 is not fulfilled. If $B_{1}, B_{2}$ and $B_{5}$ have finished their reconfiguration (indicated by $S 1$ ), the process is continued with $r_{4,3}=[F L, S R]$ and $r_{3,4}=[F L, S R]$ expressed by $S 2$. This step can be performed because all links in $\mathcal{S}\left(B_{3} B_{4}\right)$ and $\mathcal{S}\left(B_{4} B_{3}\right)$ are already using simple routing. Step $S 3$ completes the reconfiguration. Now all links are using simple routing.

Switching from Simple Routing to Flooding. Switching from simple routing to flooding $r_{i j}=[S R, F L]$ on $B_{i} B_{j}$ is controlled by $B_{i}$ which is the coordination broker in this case. Only this broker can decide whether this reconfiguration is possible due to Equation 6.2. Thus, it checks if all links in $\mathcal{P}\left(B_{i} B_{j}\right)$ are configured to use FL. If this is true, broker $B_{i}$ can initiate the reconfiguration process. In case that $B_{i}$ is a leaf broker, $\mathcal{P}\left(B_{i} B_{j}\right)$ is an empty set and $B_{i}$ can, thus, always switch its outgoing link to FL. When $B_{i}$ has decided to reconfigure $r_{i j}=[S R, F L]$, it first starts to flood notifications received 
from brokers in $\mathcal{P}\left(B_{i} B_{j}\right)$ and local clients. Additionally, $B_{i}$ drops parts of the routing entries with destination $B_{j}$ (filter list $F \operatorname{List}\left(B_{i}, B_{j}\right)$ ) and stops the processing of (un)subscriptions that $B_{j}$ is still sending. In the second step, $B_{i}$ sends a message to $B_{j}$ that let it stop sending subscriptions. This message is called StopSendingSubscriptions or shortly SSS. With the processing of this message at $B_{j}$, the reconfiguration from simple routing to flooding is completed. To inform the other broker about the successful completion the reconfiguration is acknowledged by an acknowledgement message SSSA.

Interestingly, the coordination of both mentioned reconfigurations, from FL to SR and vice versa, is done by the broker which we defined as the rendezvous broker in Section 6.3.1. Specially, it is the rendezvous broker of an arbitrary broker in $\mathcal{T}\left(B_{j} B_{i}\right)$ and a broker in $\mathcal{T}\left(B_{i} B_{j}\right)$. This means that the coordination broker is located at the border of two worlds which empowers it to make the necessary decisions. Each reconfiguration leads to the move of the rendezvous broker to its neighbor broker giving it the chance to reconfigure later.

The link reconfiguration $r_{i j}=[S R, F L]$ can also be used to reconfigure the whole overlay network. Applied to the presented example in Figure 6.4, the switching from homogeneous simple routing to homogeneous flooding would be executed as for the opposite direction just in the reverse order.

\subsubsection{Structure of Routing (Re)configurations}

In this section, we discuss the general structure of valid routing configurations and reconfiguration of certain topologies, namely a line and a star of brokers. This allows a deeper understanding of hybrid routing algorithms and provides results we built on in Section 5.4.2.

A Line of $n$ Brokers. A line of $n$ brokers contains two independent reconfiguration dimensions, where each has $n$ different valid routing configurations $[F L, F L, \ldots, F L],[F L, F L, \ldots, S R], \ldots,[S R, S R, \ldots, S R]$. Since all possible combinations are allowed, this leads to $C^{-}(n)=n^{2}$ routing configurations. Figure 6.5(a) shows all routing configurations in rectangular boxes for a line consisting of four brokers. The configuration of each link is depicted by a single digit. A value of 0 means flooding while 1 is simple routing. A pair of digits symbolizes an overlay link and the respective reverse link. Since we have four brokers and six directed link we get 6 digits in each box. Gray boxes symbolize configurations, where a leaf level (cf. Section 5.3.1) is completely reconfigured. The arrows in Figure 6.5(a) show possible one-step reconfigurations within the network. Therefore, the hamming distance between two configurations that are connected via a shortest path of length $l$ is $l$. The attached number of an arrow indicates the bit that toggles within the configuration. Each path starting from homogeneous flooding [00 0000$]$ reaching homogeneous simple routing $\left[\begin{array}{lll}11 & 11 & 11\end{array}\right]$ must contain all numbers $0, \ldots, 5$. Since there are two independent directions for reconfiguration, each configuration has two possible forward reconfigurations (configurations that tend to homogeneous routing). Exceptions are the final states with homogeneous routing and their previous states (e.g., [10 11 11]).

The number of reconfiguration paths of a line topology with $n$ brokers starting from homogeneous flooding reaching homogeneous simple routing is easy to see if we identify two reconfiguration directions $l$ and $r$. If broker $B_{l}$ and $B_{r}$ 


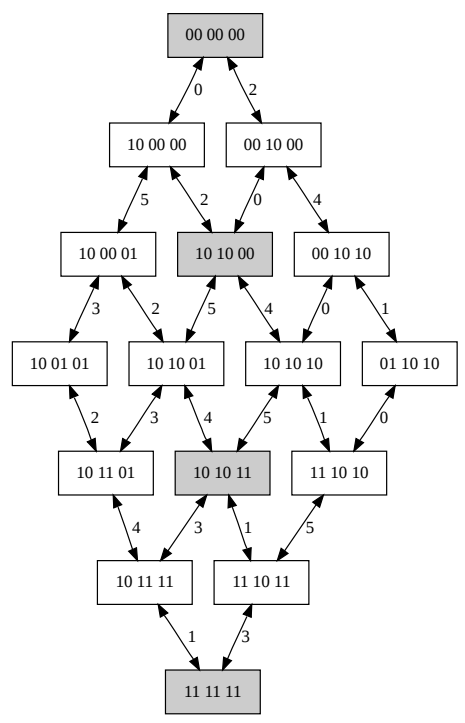

(a) 4-line

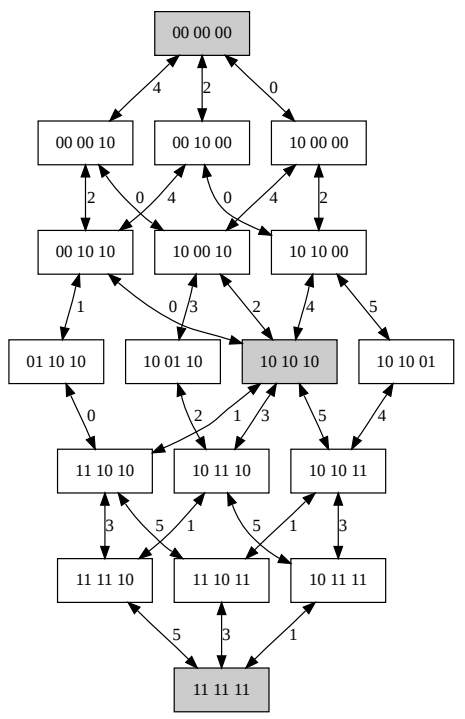

(b) 4-star

Figure 6.5: (Re)configuration networks

are the left and the right leaf brokers of the line, then a reconfiguration in $l$ means that one further reconfiguration step towards $B_{l}$ is done. Direction $r$ symbolizes one reconfiguration towards $B_{r}$. Each reconfiguration path from FL to SR consists of $n-1$ reconfigurations in $l$ and $r$ direction. Such a path can be symbolized by a path string like, e.g., "llrlrr" for a line with four brokers. It is important to realize that the positioning of all l's within the path determines the positions of $r$. Due to the independence of the directions, the number of all paths through the network equals the calculation of the number of possible path strings of length $2(n-1)$ which contain $(n-1)$ times $l$. Therefore, we get $R^{-}(n)=\left(\begin{array}{c}2(n-1) \\ n-1\end{array}\right)$ reconfiguration paths for a line of $n$ brokers.

A Star of $n$ Brokers. To calculate the number of possible configuration of a star consisting of $n$ brokers, we first recall the level-view of the topology as introduced in Section 5.3.1. In case of a star, this view consists of only two levels. The lower level $L_{0}$ contains $n-1$ level-0 leaves that are all connected to the single level-1 leaf in the upper level $L_{1}$ that is the center of the star. In opposite to a line, in a first reconfiguration step $n-1$ links can reconfigure independently. Depending on the number of reconfiguration in this first step there exist other options for further reconfigurations. In general there are $\left(\begin{array}{c}n-1 \\ i\end{array}\right)$ different configurations that $i$ out of $n-1$ are reconfigured. For each $i=$ $0, \ldots, n-1$ there are three interesting cases: (i) if $i=0, \ldots, n-3$ there is no further reconfiguration from the center to a leaf possible leading to factor 1, (ii) for $i=n-2$ exactly one link may or may not be reconfigured leading to factor 2, (iii) for $i=n-1$ all links from the center to all $n-1$ leafs are free to be reconfigured leading to $2^{n-1}$ possibilities. Putting all this together we get: 


$$
\begin{aligned}
C^{\star}(n) & =\left(\begin{array}{c}
n-1 \\
0
\end{array}\right) \cdot 1+\ldots+\left(\begin{array}{l}
n-1 \\
n-3
\end{array}\right) \cdot 1+\left(\begin{array}{c}
n-1 \\
n-2
\end{array}\right) \cdot 2+\left(\begin{array}{c}
n-1 \\
n-1
\end{array}\right) \cdot 2^{n-1} \\
& =\sum_{i=0}^{n-2}\left(\begin{array}{c}
n-1 \\
i
\end{array}\right)+(n-1)+2^{n-1} \\
& =2^{n-1}-1+(n-1)+2^{n-1} \\
& =2^{n}+n-2
\end{aligned}
$$

We see an exponential growth of the number of configurations of a star in contrast to a quadratic growth in case of a line topology. Since for $n=1,2,3$ a star and a line topology do not differ it holds that $C^{\star}(n)=C^{-}(n)$ and $R^{\star}(n)=$ $R^{-}(n)$ for $n \in\{1,2,3\}$.

The number of possible reconfiguration paths $R^{\star}(n)$ is determined as follows. We consider two distinct alternatives. One is if we have all links from the center to the leafs reconfigured after $n-1$ steps. The other is if this reconfiguration lasts $n$ steps. In Figure 6.5(b) this distinction can be seen in the central row. The gray box is the case when $n-1$ links to the leaves reconfigured in $n-1$ steps. The other black boxes are configurations where $n-2$ reconfigured and one outgoing links of the center of the star. In this case, the next reconfiguration is necessarily the missing link which can be seen on the single alternative to the next row (e.g., [ $\left.\left[\begin{array}{lll}01 & 10 & 10\end{array}\right] \rightarrow\left[\begin{array}{lll}11 & 10 & 10\end{array}\right]\right)$. For case one, we have $(n-1)$ ! different paths to the central gray box and $(n-1)$ ! different paths from the central gray box to the final configuration. For case two we have less possible paths. There are again $(n-1)$ ! paths to reach the black boxes in the same row as the central gray box. Then we have only 1 and after that $n-2, \ldots, 1$ possible paths. Adding this both cases together we get:

$$
\begin{aligned}
R^{\star}(n) & =(n-1) ! \cdot(n-2) !+(n-1) ! \cdot(n-1) ! \\
& =(n-2) ! \cdot[(n-1) !+(n-1) \cdot(n-1) !] \\
& =(n-2) ! \cdot(n-1) ! \cdot(1+n-1) \\
& =n ! \cdot(n-2) !
\end{aligned}
$$

Again the number of reconfiguration paths is much higher in case of a star than it is in case of a line of brokers.

For each topology such a (re)configuration network as depicted in Figure 6.5 can be generated. They quickly become complicated as for example for a binary tree with only three levels which we depict in Appendix B. Often it is hard to derive explicit analytic results for the number of configurations and the reconfiguration paths. Nevertheless, in this section we derived the two extreme values of topology consisting of $n$ brokers, the line with the minimal number of valid routing configurations and the star with the maximum. Both values were the foundation of estimating the optimization complexity of the capacity planning in Section 5.4.2. 


\subsection{Self-optimizing Algorithm}

So far we focused on the question how we can reconfigure single overlay links of a hybrid routing algorithm in a way that it does not violate the correctness of the publish/subscribe system. Before having this reconfigurability, the routing algorithm was a design parameter. In the latter section it has been converted into a variable to manage the system. Following, we go a step further and add adaptive resp. self-optimizing behavior to the system. Thus, we show how a self-optimizing routing or adaptive routing (AR) based on local decisions can be realized.

\subsubsection{Optimization Criterion}

A (self-)optimization always requires a cost measure. In Chapter 4, we introduced a bunch of them as outputs of the stochastic system model. There, we mainly considered the global measures. Since the target here is to develop a decentralized algorithm, which has as less interactions as possible, we must consider a measure that is easy to determine by one of the brokers $B_{i}$ or $B_{j}$ that share one overlay link $B_{i} B_{j}$. Our approach here is to define the costs per time of such an overlay links as follows:

$$
C_{i j}=c_{i j} \cdot r_{i j}^{n}+c_{j i} \cdot r_{i j}^{s}
$$

This cost measure $C_{i j}$ is the weighted sum of the traffic of notifications $r_{i j}^{n}$ and reverse subscriptions $r_{i j}^{s}$ occurring on the overlay link $B_{i} B_{j}$. This reverse counting of $r^{n}$ and $r^{s}$ is similar to $\nu_{\text {out }}^{n}$ and $\nu_{\text {out }}^{s}$ introduced in Chapter 4 . We use other symbols to distinguish between analytic values and measured ones. The unitless weights $c_{i j}$ and $c_{j i}$ represent the costs for forwarding and processing one message. They help us to express asymmetric links, different costs for notifications and subscriptions, and finally, to express costs for special, e.g., expensive overlay links. In case that $c_{i j}=c_{j i}=1$, the costs $C_{i j}$ are equivalent to the traffic of notifications and subscriptions. Depending on the optimization objectives, like optimizing network performance or load balancing, these coefficients can be derived from metrics like CPU processing time, memory consumption, or forwarding latency.

Adaptive routing tries to reduce costs of arbitrary overlay links $B_{i} B_{j}$ by adapting the routing algorithm. Since the global costs are the sum of the costs of all links, the reduction of the costs of one link reduces the global costs, too. Even indirectly connected performance measures such as the global message delays $\Delta^{n}$ and $\Delta^{s}$ are improved. This of course requires that a reconfiguration of the routing algorithm on one link does not influence other links negatively. In Section 6.4.2, we argue that this is really the case when we use only flooding and simple routing for adaptive routing.

To decide weather a valid reconfiguration of the routing algorithm of a link is reasonable, the current $C_{i j}$ costs have to be higher than the expected costs $\hat{C}_{i j}$ after a reconfiguration. To determine the expected costs, the expected traffic after a reconfiguration, i.e., $\hat{r}_{i j}^{n}$ and $\hat{r}_{i j}^{s}$, have to be predicted. How this is done based on incoming messages is discussed in the following for both reconfigurations $r_{i j}=[F L, S R]$ and $r_{i j}=[S R, F L]$. Furthermore, we derive an optimization criterion for both cases. 
Flooding to Simple Routing. While using flooding on link $B_{i} B_{j}$, broker $B_{i}$ sends all notifications to $B_{j}$ without considering $B_{j}$ 's interests. This can lead to a high amount of notifications that $B_{j}$ has to discard because neither $B_{j}$ 's local subscribers nor one of its successing neighbors in $\mathcal{S}\left(B_{i} B_{j}\right)$ are interested in those notifications. If the number of discarded messages exceeds a certain level, it is reasonable to reconfigure the link configuration to simple routing, i.e., to send subscriptions to $B_{i}$ and, therefore, to reduce the number of notifications. The current $\operatorname{costs} C_{i j}$ of flooding only consist of the notification traffic $r_{i j}^{n}$. Both brokers can easily measure it. In order to compute the expected costs $\hat{C}_{i j}$ that will occur in simple routing, the traffics $\hat{r}_{i j}^{n}$ and $\hat{r}_{i j}^{s}$ must be determined. Only broker $B_{j}$ can directly measure these traffics. On the one hand, the expected subscription traffic $\hat{r}_{i j}^{s}$ equals the sum of the local subscription birth rate and the aggregated subscription traffic from all successing links $\mathcal{S}\left(B_{i} B_{j}\right)^{1}$. On the other hand, the expected notification traffic $\hat{r}_{i j}^{n}$ can easily be derived since the broker is already matching notifications before forwarding them to neighbors. Thus, $\hat{r}_{i j}^{n}$ is the notification traffic which comes along $B_{i} B_{j}$ and is forwarded to $\mathcal{S}\left(B_{i} B_{j}\right)$ or a local client of $B_{j}$. This traffic is always smaller than or equal to the current notification traffic $r_{i j}^{n}$. The ratio $\hat{r}_{i j}^{n} / r_{i j}^{n}$ is called the effective forwarding probability $\hat{P}^{n}\left(B_{i} B_{j}\right)$ which has a slightly different meaning compared to $P^{n}\left(B_{i} B_{j}\right)$ (cf. Section 4.4.7). Finally, we get the following optimization criterion for a reconfiguration $r_{i j}=[F L, S R]$ :

$$
c_{i j} \cdot r_{i j}^{n}>c_{i j} \cdot \hat{r}_{i j}^{n}+c_{j i} \cdot \hat{r}_{i j}^{s}
$$

If the costs for the flooding of notifications is greater than the expected costs for sending filtered notifications and subscriptions, the reconfiguration is reasonable. When substituting $\hat{P}^{n}\left(B_{i} B_{j}\right)$, the reconfiguration criterion looks as follows:

$$
\begin{aligned}
c_{i j} \cdot\left[1-P^{n}\left(B_{i} B_{j}\right)\right] \cdot r_{i j}^{n} & >c_{j i} \cdot \hat{r}_{i j}^{s} \\
c_{i j} \cdot r_{i j}^{\bar{n}} & >c_{j i} \cdot \hat{r}_{i j}^{s}
\end{aligned}
$$

This formulas point out that interesting notifications actually can be skipped, but not the false positives $\bar{n}$. Only if the costs of the false positive notifications (left hand-side of the equation) exceed the costs for the subscription traffic a reconfiguration should be performed. For the implementation this result is important since for a reconfiguration decision only two counters are needed instead of three.

Simple Routing to Flooding. If simple routing is applied on the link $B_{i} B_{j}$, broker $B_{j}$ sends subscriptions to $B_{i}$ that builds up a routing table to filter the notifications forwarded to $B_{j}$. If $B_{j}$ is interested in almost all notifications forwarded by $B_{i}$ or the amount of subscription messages is significantly higher than the one of notifications a reconfiguration is reasonable.

As before, the current $\operatorname{costs} C_{i j}$ of simple routing can easily be determined by both brokers $B_{i}$ and $B_{j}$ by measuring $r_{i j}^{n}$ and $r_{i j}^{s}$. To compute the expected

${ }^{1}$ All links in $\mathcal{S}\left(B_{i} B_{j}\right)$ do use SR according to Equation 6.1. 
costs $\hat{C}_{i j}$ after a reconfiguration to FL, it is only necessary to predict the notification traffic $\hat{r}_{i j}^{n}$ since $\hat{r}_{i j}^{s}=0$. The information about the expected flooding traffic is only available on broker $B_{i}$ because it is receiving all unfiltered notifications from $\mathcal{P}\left(B_{i} B_{j}\right)$ and of its local clients. This accumulated traffic will appear on the overlay link $B_{i} B_{j}$ after the reconfiguration $r_{i j}=[S R, F L]$. Thus, the reconfiguration criterion is the reverse formula to the inverse reconfiguration (cf. Equation 6.4):

$$
\begin{array}{r}
c_{i j} \cdot r_{i j}^{n}+c_{j i} \cdot r_{i j}^{s}>c_{i j} \cdot \hat{r}_{i j}^{n} \\
c_{j i} \cdot r_{i j}^{s}>c_{i j} \cdot \hat{r}_{i j}^{\bar{n}}
\end{array}
$$

If the expected costs for the flooding of notifications is smaller than the costs for sending filtered notifications and subscriptions, the reconfiguration is reasonable. This is equivalent to: if the expected costs for the false positives notifications of flooding is smaller than the costs for forwarding subscriptions, the reconfiguration is reasonable.

Interestingly, both reconfiguration criteria can be evaluated by the broker that is the coordination broker of the routing reconfiguration as described in the latter section. Thus, it can be seamlessly integrated into a self-optimization algorithm. Before doing this, we briefly point out an interesting relation to Chapter 4 .

Effective Forwarding Probabilities. The central quantities of the derivation of the stochastic system model are the forwarding probabilities of messages (cf. Section 4.4.7). As mentioned, we distinguish between two types of probabilities for different message types $x$ : the normal probabilities $P^{x}\left(B_{i} B_{j}\right)$ and the effective probabilities $\hat{P}^{x}\left(B_{i} B_{j}\right)$. To determine the mathematical relation between both, we use the formula from the latter paragraph which is $\hat{P}^{n}\left(B_{i} B_{j}\right)=\hat{r}_{i j}^{n} / r_{i j}^{n}$. In terms of the traffic provided in Section 4.4.8, $\hat{r}_{i j}^{x}$ corresponds to $\nu_{\text {out }}^{x}\left(B_{i} B_{j}\right)$ and $r_{i j}^{x}$ to $\nu_{i n}^{x}\left(B_{i} B_{j}\right)$, where $x=n$ in case of notifications. When we further substitute $\nu_{\text {out }}^{x}$ by its defining formula we get:

$$
\begin{aligned}
\hat{P}^{x}\left(B_{i} B_{j}\right) & =\frac{\nu_{o u t}^{x}\left(B_{i} B_{j}\right)}{\nu_{i n}^{x}\left(B_{i} B_{j}\right)} \\
& =P^{x}\left(B_{i} B_{j}\right) \cdot \frac{\lambda_{a c c}^{x}\left(B_{i} B_{j}\right)}{\nu_{i n}^{x}\left(B_{i} B_{j}\right)}
\end{aligned}
$$

Due to $\lambda_{a c c}^{x}\left(B_{i} B_{j}\right) \geq \nu_{i n}^{x}\left(B_{i} B_{j}\right)$ it holds $\hat{P}^{x}\left(B_{i} B_{j}\right) \geq P^{x}\left(B_{i} B_{j}\right)$. In adaptive routing brokers measure $\hat{P}^{x}\left(B_{i} B_{j}\right)$ by counting $\nu_{\text {out }}^{x}\left(B_{i} B_{j}\right)$ and $\nu_{i n}^{x}\left(B_{i} B_{j}\right)$. Since $\lambda_{a c c}^{x}\left(B_{i} B_{j}\right)$ is unknown for the broker, it cannot determine $P^{x}\left(B_{i} B_{j}\right)$. For the analysis, we can determine both values.

\subsubsection{Algorithm Details}

In this section, we focus on an algorithm that implements adaptive routing. First, we provide a pseudocode of the algorithm that combines all concepts introduced before. Later we explain its major properties including the optimality 


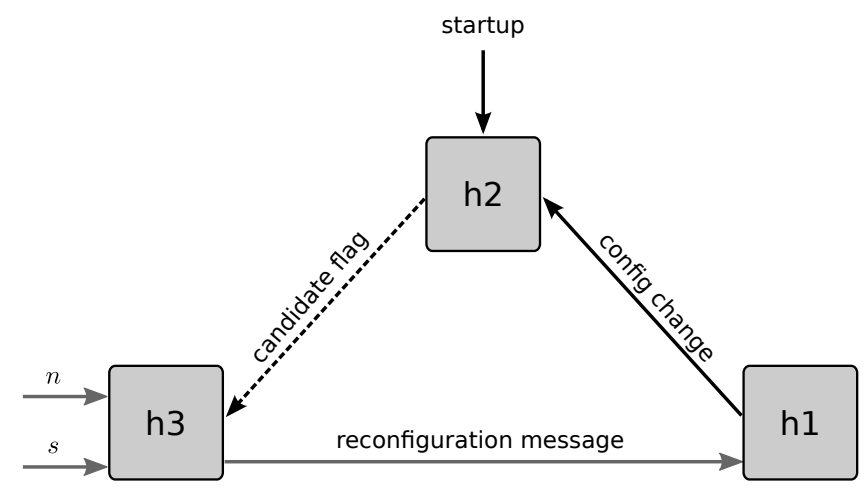

Figure 6.6: Adaptive routing algorithm

of the algorithm. Furthermore, we discuss important issues concerning overlay reconfigurations and the connection of clients. For shortness we call the algorithm adaptive routing abbreviated by $A R$.

Verbal Pseudocode. The algorithm we describe here, is executed on each broker in the publish/subscribe system. Following one central theme of this thesis, the design of this distributed algorithm is even-driven as much as possible. Thus, the code consists of several event handlers for different types of internal and external events.

H1: First, every broker implements routines that handle reconfiguration messages (SSS, SFN and acknowledgements) that have been introduced to coordinate the reconfiguration process. They are handled according to the scheme as we described in Section 6.3.3. Furthermore, when the handling of SSS resp. SFN has finished an internal configuration change event is triggered.

H2: When a configuration change event occurs, the broker determines potential reconfigurations of connected overlay links where it can be the coordination broker. Configuration change events also occur at startup on all brokers. Depending on the current routing algorithm, for a link $B_{i} B_{j}$ with a potential reconfiguration either all links in $\mathcal{S}\left(B_{i} B_{j}\right)$ must use simple routing or all links in $\mathcal{P}\left(B_{i} B_{j}\right)$ must use flooding (cf. Equations 6.1 and 6.2). All links of potential reconfigurations are marked by a candidate flag.

H3: The last routine handles incoming notifications and subscriptions. This traffic monitoring is done in order to determine the costs. For each overlay link that has been marked by the candidate flag there are two necessary counters for incoming subscriptions and not interesting notifications. Each incoming message updates the related traffic measure. To reduce noise, these traffics are smoothened by a low-pass filters for example. Based on the traffic, the broker checks if the reconfiguration is reasonable. This is the case if the predicted costs are lower than the current costs (cf. Equations 6.4 and 6.5). To avoid oscillation in the optimization process, a reconfiguration is only triggered if the gain is higher than a predefined threshold $t r$. For each reasonable reconfiguration, the reconfiguration process is triggered as described in Sect 6.3.3.

Figure 6.6 shows the described event handlers H1, H3 and H3. Each handler deals with incoming (internal and external) events depicted by arrows. The 
dashed arrow symbolizes that the candidate flag is no real event although it could be implemented so. With the gray arrows we marked messages that transport external events between different brokers. Especially, notifications and subscriptions coming from outside the system drive the adaptive routing algorithm to internal state changes and further event publishing. With this implementation of the adaptive routing algorithm, we internally apply the event-based system approach with its inherent advantages. Thus, we get a real distributed event-based system also on algorithm level.

Major Properties. The described algorithm has the following major properties: (i) it is completely distributed, (ii) it requires only local knowledge, (iii) it does not depend on a complex interaction scheme, and (iv) it introduces only little additional traffic. The first property stems from the fact that the algorithm works independently on all brokers in the distributed publish/subscribe system. This means, that each broker may trigger a reasonable reconfiguration which leads to possibly simultaneously reconfigurations. The second property (ii) can directly be derived from the discussion of local optimizations in Section 6.4. There, we discussed how arbitrary brokers can determine potential (based on the routing configuration of the neighbors) and reasonable reconfigurations (based on local measurements of traffic). Properties (iii) and (iv) result from the described reconfiguration and optimization process in Section 6.3.3. There, we showed that any reconfiguration is processed by first evaluating the reconfiguration condition, second by changing the local behavior, and third by sending a signal to neighboring brokers. Besides these signaling messages, the only additional messages that have to be sent are the messages required to build up the routing table when switching a link from flooding to simple routing. If the time between consecutive reconfigurations from flooding to simple routing is not shorter than the average subscription lifetime, no additional messages compared to simple routing are needed. Furthermore, there is no need of additional coordination since reconfigurations are done link by link and there is no possible configuration, where neighbor brokers will reconfigure the same link at the same time: If flooding is active on a link $B_{i} B_{j}$, only broker $B_{j}$ is able to trigger a reconfiguration. If simple routing is used, only $B_{i}$ is able to initialize a reconfiguration. Therefore, the adaptive routing algorithm assures a conflict-free self-optimization of the system.

Local Optimality. Interestingly, the reconfiguration of a link does not influence the message traffic on other links. This can be shown by examining a reconfiguration $r_{i j}=[F L, S R]$. We know that all links in $\mathcal{S}\left(B_{i} B_{j}\right)$ use simple routing and all links in $\mathcal{P}\left(B_{i} B_{j}\right)$ use flooding, otherwise the correctness criterion given by Equations 6.1 resp. 6.2 would be violated before or after a reconfiguration. In between, $B_{i} B_{j}$ switches to simple routing and subscriptions are sent to $B_{i}$, but they are not forwarded to the links in $\mathcal{P}\left(B_{i} B_{j}\right)$ because they use flooding. Furthermore, $B_{i}$ filters incoming notifications before forwarding them to $B_{j}$, but there is no notification that appears additionally on links in $\mathcal{S}\left(B_{i} B_{j}\right)$ because $B_{i}$ filters them already according to their interests. These two facts show that $r_{i j}=[F L, S R]$ does not influence other link costs. Similarly, we can argue for $r_{i j}=[S R, F L]$. Based on this knowledge, we can state that a reconfiguration affects only the traffic of notifications and subscriptions on an individual 


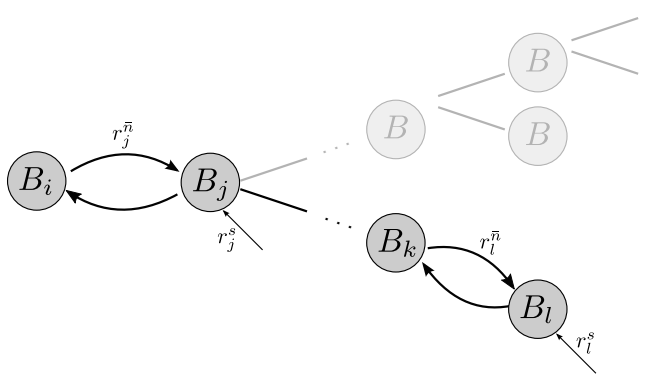

(a) Evaluation setup

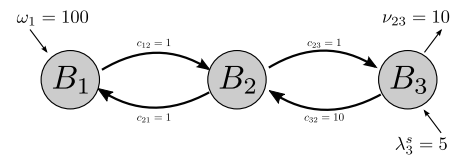

(b) Counter example

Figure 6.7: Optimality of adaptive routing

link. Therefore, a cost reduction on a particular link results in reduced global costs, too. Since in static situations such local optimizations are repeated until no further reconfigurations are profitable, the global cost measure can be reduced in each step of the self-optimization. Due to this greedy behavior, the system reaches a local optimum according to the introduced cost measure and the manipulation of the routing configuration. If also a global optimum can be reached is discussed in the following paragraph. Of course, in highly-dynamic situations the algorithm may introduce overhead which is not amortized.

Global Optimality. Adaptive routing is not only local optimal but often global optimal as well. With global optimality we mean that in static situations reconfigurations lead to that routing configuration of all valid configurations that is the globally best according to the introduced cost measure. To proof this claim, we check if a reasonable reconfiguration of a link $r_{i j}=[F L, S R$, is not prohibited by another reconfiguration $r_{k l}=[F L, S R$, ] that cannot take place due to the local decision. We start with an overlay network that uses flooding on all links. For our proof, we analyze any two connected brokers $B_{i}$ and $B_{j}$ and any two other connected brokers $B_{k}$ and $B_{l}$ that are part of the subtopology $\mathcal{T}\left(B_{i} B_{j}\right)$. This situation is depicted in Figure 6.7(a). We assume that $B_{k} B_{l}$ does not switch to simple routing due to the optimization criterion. This inhibits all following reconfigurations on the path to $B_{i} B_{j}$ because $\mathrm{SR}$ must follow SR. Thus, also $B_{i} B_{j}$ cannot switch to simple routing. Now we try to find out if the decision for flooding at $B_{k} B_{l}$ inhibits a possible (greater) cost reduction at $B_{i} B_{j}$. This reduction can only be realized if against the proposed algorithm all links in the subtopology switch to simple routing. For this purpose we slightly change the meaning of the symbols: $r_{j}^{s}$ and $r_{l}^{s}$ symbolize the traffic of subscriptions that are received at $B_{j}$ resp. $B_{l}$. In simple routing, they have to be sent to $B_{i}$ resp. $B_{k} \cdot r_{j}^{\bar{n}}$ and $r_{l}^{\bar{n}}$ express the traffic of false positive notifications that $B_{j}$ resp. $B_{l}$ is not interested in during flooding. With this definitions we get the first inequality for $B_{k} B_{l}$. Since we assume the costs for sending subscriptions is higher than getting false positive notifications this link stays in flooding. Thus,

$$
c_{l k} \cdot r_{l}^{s}>c_{k l} \cdot r_{l}^{\bar{n}}
$$


For $B_{i} B_{j}$ we have the inverse inequality because we assume that this link could reduce the cost measure if all links in the subtopology would have been reconfigured to simple routing. Thus,

$$
c_{j i} \cdot r_{j}^{s}<c_{i j} \cdot r_{j}^{\bar{n}}
$$

There are relations between the traffics in these inequalities. All subscriptions that are sent via $B_{k} B_{l}$ will appear also on $B_{i} B_{j}$. Additionally, subscriptions from other brokers may be received at $B_{j}$ :

$$
r_{j}^{s} \geq r_{l}^{s}
$$

On the other hand, the set of false positive notifications for $B_{l}$ contains all false positive notifications of $B_{j}$ plus some additional messages:

$$
r_{l}^{\bar{n}} \geq r_{j}^{\bar{n}}
$$

After several transformations and substitutions, we can combine the inequalities in the following way:

$$
\frac{c_{k l}}{c_{l k}}<\frac{r_{j}^{\bar{n}}}{r_{j}^{s}} \leq \frac{r_{l}^{\bar{n}}}{r_{j}^{s}}<\frac{c_{k l}}{c_{l k}} \cdot \frac{r_{l}^{s}}{r_{j}^{s}} \leq \frac{c_{k l}}{c_{l k}}
$$

If there are non-negative assignments for the traffics and the cost coefficients that fulfill the inequality, then $B_{k} B_{l}$ inhibited that $B_{i} B_{j}$ could switch to reduce its local costs. It does not mean that the global costs will be reduced, but this inequality is a sine qua non. An example when adaptive routing does not result in a global optimal routing configuration is shown in Figure 6.7(b). There, the overlay link $B_{2} B_{3}$ is asymmetric in its costs. Starting point is flooding. $B_{3}$ is interested in $10 \%$ of the 100 messages per second that are published at $B_{1}$. This interest is defined by a flow of 5 subscriptions per second. Due to the high costs of $c_{32}$ broker $B_{3}$ will not decide to use simple routing $(1 \cdot 90<10 \cdot 2 \cdot 5=100)$. Nevertheless, at $B_{2}$ a reconfiguration to SR would be beneficial $(1.90>2 \cdot 1 \cdot 5)$. A reconfiguration of $B_{1} B_{2}$ would lead to a benefit of 80 cost units per time which more than compensates the increased cost $(+10)$ of the other link. But this optimization possibility is not materialized because $B_{2} B_{3}$ inhibits it.

Interestingly, there are special values of the costs coefficients for which the Inequality 6.6 is false independently from the selected traffics, namely if $\frac{c_{k l}}{c_{l k}} \geq \frac{c_{i j}}{c_{j i}}$ holds. Then, the rates do not play a role and the adaptive routing converges to a global optimum since all beneficial reconfigurations can take place. This result is only valid for one overlay link and for one direction of the reconfiguration. If we wish that adaptive routing is optimal for all reconfigurations in a publish/ subscribe network, then the restrictions for the cost coefficients are that each link must have symmetric costs $c_{i j}=c_{j i}$. In this case, the final costs are equal or less than that of homogeneous routing. The restriction that $c_{i j}=c_{j i}$ seems rather strong but it is often met in networks like LANs. If the restriction cannot be hold, adaptive routing can reach a global optimum but could also stuck in a local optimum. 


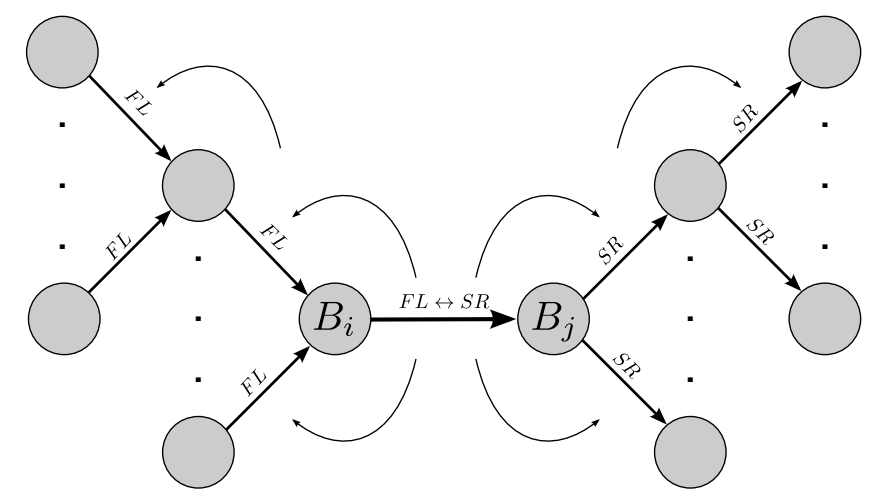

Figure 6.8: Connection of two overlays

Overlay Reconfigurations. So far we investigated already existing static overlays network. Practically, an overlay changes over time. For example, at startup we begin with a single broker. Step by step other brokers are connected to already active ones until the complete network is up. A shutdown of the network is processed in the reverse way which requires that the removal of brokers has to be supported. Furthermore, sometimes the substitution of brokers is required or the fusion of two overlays. Following, we analyze which implications such overlay reconfiguration have on the routing configuration. Thus, we only consider the correctness, but skip the self-optimizing property of adaptive routing. After the completion of such a reconfiguration the AR will again push the system to an optimized operation point. In this section, we especially want to examine the addition and the removal of single overlay links. These two basic reconfigurations include all before mentioned reconfigurations.

The removal of a single link does not play any role for the correctness of the routing configuration because it only removes dependencies between links but does not introduce new ones. This practically means that the removal of a leaf broker or the separation of a network does not require any additional treatment for the adaptive routing level. Of course on the level of the notification routing tables, unnecessary filters should be removed.

A more interesting overlay reconfiguration is the establishment of a new connection between two brokers. In Figure 6.8 we exemplary depicted the creation of a new overlay link between brokers $B_{i}$ and $B_{j}$ which connects two separate subtopologies $\mathcal{T}\left(B_{i} B_{j}\right)$ and $\mathcal{T}\left(B_{j} B_{i}\right)$, where each network uses adaptive routing. Obviously, if one subtopology contains only one broker $\left(\mathcal{T}\left(B_{i} B_{j}\right)=\left\{B_{j}\right\}\right.$ or $\left.\mathcal{T}\left(B_{j} B_{i}\right)=\left\{B_{i}\right\}\right)$, the creation of a link equals to the connection of a single broker to an already existing topology. When connecting the two subtopologies, it must be decided which routing configuration $r_{i j}$ and $r_{j i}$ the new link must have. The answer to this question can be derived from the application of the correctness definitions given by Equations 6.1 and 6.2. For the sake of simplicity, we consider here only one direction of the link $B_{i} B_{j}$. The routing configuration $r_{i j}$ depends on the routing configurations of links in $\mathcal{S}\left(B_{i} B_{j}\right)$ and $\mathcal{P}\left(B_{i} B_{j}\right)$. If there is a FL link in $\mathcal{S}\left(B_{i} B_{j}\right)$, the new link must also use flooding. This demand can be checked by broker $B_{j}$. In case of a SR link in $\mathcal{P}\left(B_{i} B_{j}\right)$ (checked by $\left.B_{i}\right)$, the new link must also use simple routing. In other cases, the selection 


\begin{tabular}{|c||c|c|c|}
\hline$B_{i} / B_{j}$ & FL & SR & $*$ \\
\hline \hline FL & FL & conflict & FL \\
\hline SR & conflict & SR & SR \\
\hline$*$ & FL & SR & FL / SR \\
\hline
\end{tabular}

Table 6.2: Compatible configurations

of the routing configuration of the new link is free ("*"). To find consistent configurations for $r_{i j}$ and $r_{j i}$, the brokers negotiate them when they connect.

This is done before the routing algorithms become active, i.e., before they start to send notifications and subscriptions. The negotiation requires the mutual sending of one adaptive routing connect message $A R C$ that contains the demand for the new link configurations $\tilde{r}_{i j}$ and $\tilde{r}_{j i}$ whose values may be $F L, S R$ or " $*$ " for a free choice of the routing configuration. When the broker on the opposite site receives $A R C$, it compares its own demands on the routing configuration with the one from the neighbor. For one direction the possible combinations are depicted in Table 6.2. This view is consistent for both brokers. There are six combinations with a straight forward decision. This is, if one broker requires $F L$ resp. $S R$ and the other requires the same or has no demands on the new link configuration. If both brokers do not have any demands on the new link configuration, "*/*", a predefined preferred algorithm is used. This is to achieve a consistent decision on both brokers in one step.

The most complicated situation occurs if there are conflicting demands on the routing configuration of the new link. In case of connecting a single broker to the overlay, this situation never occurs since the new broker has no demands for the new link and, thus, always sends an $A R C$ message with "*/*". Due to this reason, a stepwise creation of a topology by adding one broker after the other is possible. If two proper topologies are connected, the conflict can be solved in two ways. The easiest way is to refuse the connection. If this is not acceptable, cascading reconfigurations may occur. Cascading reconfigurations are a sequence of reconfigurations that is propagated through parts of the network to allow the new link to be inserted. In the worst case, one complete subtopology has to change its routing configuration.

In Figure 6.8 possible cascading reconfigurations after a connection of broker $B_{i}$ and $B_{j}$ are marked. Depending on the choice of the algorithm in a conflicting situation on $B_{i} B_{j}$ either links in the left subtopology have to be reconfigured to simple routing or links in the right subtopology to flooding if they have no appropriate configurations. The cascading reconfigurations can be implemented by introducing a reconfiguration request message $A R R$ containing a routing configuration. It allows brokers to initiate a reconfiguration even if there is no reason in terms of optimization. If a broker receives message $A R R$, it either starts to reconfigure the related link or it further sends reconfiguration requests to its neighbor brokers if this is necessary to reach a correct routing configuration. In the latter case, the sending broker waits until the related links are reconfigured before proceeding the originally requested reconfiguration. This way, $A R R$ disseminates through the network until it reaches reconfigurable overlay links. Thus, in the inverse order the pending reconfigurations can be carried out until the originating new overlay link is reached. This behavior is similar 


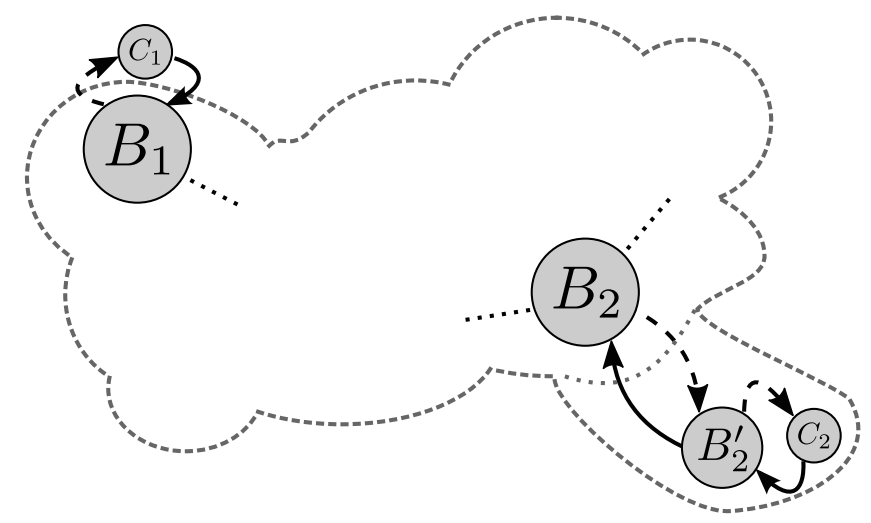

Figure 6.9: Connecting clients

to the waves of the echo algorithm by Chang [25].

Beside the connection of topologies the described approach can also be used to allow (manual) reconfigurations of the routing of any link. The implementation of both concepts, the negotiation about the routing configuration and the cascading reconfigurations, can be easily integrated into the adaptive routing algorithm by adding handling routines for both message types $A R C$ and $A R R$ and an event handler for new connections.

Connecting Clients. Depending on the individual implementation of the publish/subscribe middleware, there are two ways to connect clients to an overlay network which are both illustrated in Figure 6.9. From the functional point of view it does not matter but internally differences arise.

One possibility is to have local clients which "live" in the same context as a full broker [74]. The client communicates locally with the broker without a need of message passing. The broker filters incoming notifications according to the filters of the client and receives all published notifications of the client. Since the broker itself has full functionality all kinds of routing algorithms can be supported. For the forwarding of notifications and control messages, brokers treat clients specially, i.e., a control message is never forwarded to a local client. In Figure 6.9, client $C_{1}$ is hosted by full broker $B_{1}$. In terms of hybrid routing, the routing behavior between $C_{1}$ and $B_{1}$ is FL and SR for the reverse direction.

It is also possible to have remote clients which are a light-weight brokers with reduced functionality, i.e., they handle only one neighbor and do not support complex routing algorithms. Also the reconfiguration of the routing algorithm is not supported. The standard behavior of a light-weight broker is to flood subscriptions and notifications to the connected full broker. In Figure 6.9, client $C_{2}$ is hosted by the light-weight broker $B_{2}^{\prime}$. Light-weight broker $B_{2}^{\prime}$ is connected via $\mathrm{FL}$ and SR with the full broker $B_{2}$. This way $B_{2}^{\prime}$ transfers the algorithms of its local clients. By doing so, the routing algorithms applied at broker $B_{2}$ are much simpler because there is only one type of destinations - brokers. The only thing that has to be additionally implemented at $B_{2}$ is the possibility to inhibit the reconfiguration of links to light brokers by adaptive routing. This information could be exchanged when the overlay link is established and can be 


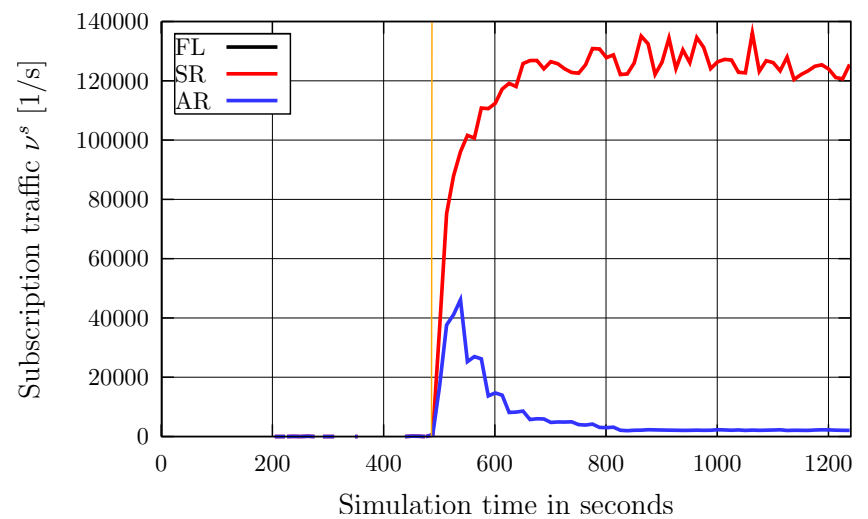

Figure 6.10: Step response of the subscription traffic

stored by a link related flag in the internal data structure. This feature of a temporal or permanent inhibition of reconfigurations could also be reasonable in other situations.

In both considered approaches the connection between the broker and the client resp. light-weight broker is configured as a combination of flooding and simple routing. This way it does not influence the correctness of the system according to Equations 6.1 resp. 6.2.

\subsection{Evaluation}

Since we have a system model which also includes hybrid routing (cf. Section 6.3.2) we are able to evaluate traffic measures for different routing configurations. Based on a large range of experiments we proofed the conformity of simulation results and the analysis (cf. Section 4.5.1) which are both based on the same assumptions. Nevertheless, adaptive routing includes more than hybrid routing, namely, adaptivity. Thus, we decided to use a discrete event simulation to evaluate adaptive routing. With a simulation we are able to evaluate the behavior of adaptive routing in dynamic situation with changing subscription birth rates for example.

Generally, our evaluation consists of the analysis of a step response of the system and the simulation of the system behavior in dynamic situations. For brokers we set a capacity of $c(B)=1400 \mathrm{~s}^{-1}$ and for physical links $c(\bar{l})=$ $1200 s^{-1}$. Physical links are mapped one to one to overlay links, i.e., each overlay link consists of one physical link and physical links are connected in the same way as overlay links. The service time on brokers and links is exponential distributed with an expected value of $1 / c$. The basic topology for the experiments consists of a complete binary tree with 127 brokers. For both experiments we define costs of 1 cost unit per message.

\subsubsection{Step Response}

In system theory, the step response plays a central role. It shows the systems reaction on sudden changes of input values. From the practical standpoint, 


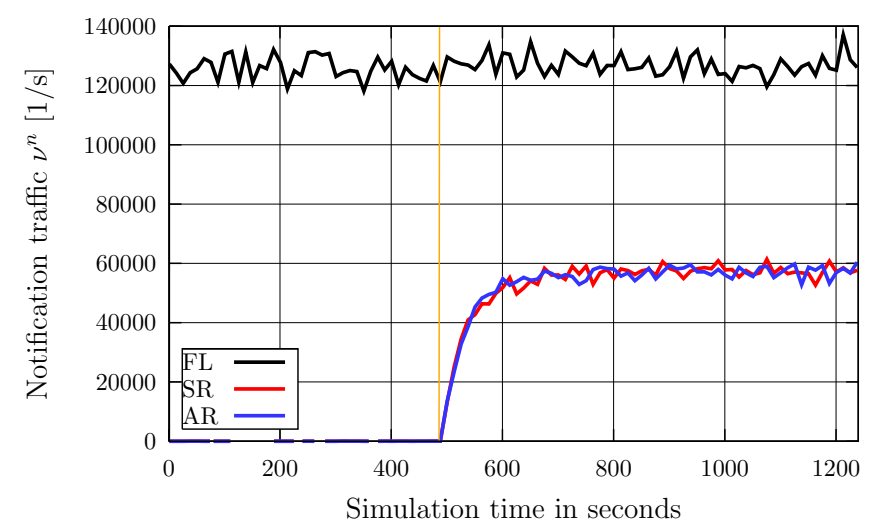

Figure 6.11: Step response of notification traffic

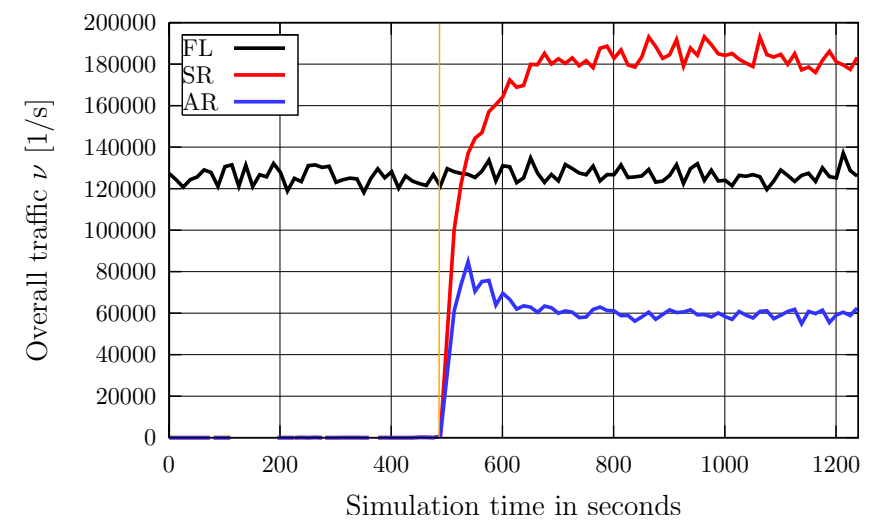

Figure 6.12: Step response of the overall traffic 
it shows the system reaction in extreme situations that could be oscillations or even instability. Furthermore, the step response provides insights of the dynamics such as the reaction time and the settling time [62].

For our experiment, we set a global publication rate of $1000 \mathrm{~s}^{-1}$ uniformly divided over all brokers, 1000 filter classes and a subscription lifetime of 60 seconds. The threshold of the adaptive routing algorithm (cf. Section 6.4.2) is set to $t r=5 \%$, meaning that each broker performs reconfigurations that are at least $5 \%$ better than the current one. Figures 6.10,6.11 and 6.12 depict the step response of the system for a step of the global subscription birth rate from $0 s^{-1}$ to $500 \mathrm{~s}^{-1}$ at $t=487 \mathrm{~s}$. The local subscription birth rate at each broker is the fraction of the global rate by the number of brokers and the number of filter classes. The figures show the traffic for different routing algorithms, namely flooding, simple routing and adaptive routing. This experiment is based on a single run which can be recognized by the fluctuation of the depicted traffics that are measured in a one second interval.

In Figure 6.10 the total subscription traffic is depicted. Clearly, in case of flooding the traffic is always zero since this algorithm does not forward subscriptions at all. In contrast, with simple routing which is subscription and unsubscriptions flooding on all overlay links, the subscription traffic converges to approximately $126,000 \mathrm{~s}^{-1} \mathrm{~s}$. The blue graph shows the adaptive behavior of adaptive routing starting in a homogeneous simple routing configuration. The sharp increase of the subscription traffic caused by more and more issued subscriptions, is damped by the reconfiguration of the routing algorithm of overlay links. Stepwise, starting from the lowest level of the tree $\rightarrow \overrightarrow{\mathbb{L}}_{0}$ (cf. Section 5.3.1) links are reconfigured to flooding since the subscription traffic exceeds the traffic of false positive notifications. The reduction effect is stronger at the beginning with a drop of about $40 \%$ in the subscription traffic which has two reasons. First, the number of brokers involved in this reconfiguration is 64 which is almost half of all brokers. Second, links in $\neg \overrightarrow{\mathbb{L}}_{0}$ transmit all issued subscriptions in the network despite the ones published at the connected leaf brokers. Further reconfigurations on higher levels do not have such a great effect but still have a gain greater than $t r=5 \%$ per link. The reconfiguration process ends about 350 seconds after the step in the subscription birth rate. The steady value of the subscription traffic of about $2,190 \mathrm{~s}^{-1}$ indicates that almost all levels despite $\overrightarrow{\mathbb{L}}_{1}$ and $\overrightarrow{\mathbb{L}}_{0}$ have been reconfigured. The final state is significantly better then homogeneous simple routing and is very close to homogeneous flooding according to the subscription traffic.

In Figure 6.11 the total notification traffic of the different routing algorithms is depicted. Clearly, the step of the subscription rate does not influence the notification traffic generated by flooding which oscillates around $126,000 \mathrm{~s}^{-1}$. This is the number of overlay links times the global publication rate. Both, simple routing and adaptive routing do not generate any notification traffic before the step of the subscription birth rate, because there is no interest for publications in the network and, thus, no subscriptions and notifications are forwarded. After the step of the subscription birth rate, the global notification traffic for both algorithms exponentially converges to a traffic of approximately $60,000 \mathrm{~s}^{-1}$ which is a little less than half of the value of flooding. This value is determined by the average number of subscriptions, their spatial distribution and the network topology. Interestingly, the notification traffic of AR follows the traffic of SR which means that on reconfigured links, i.e., links that use FL, the flow of no- 


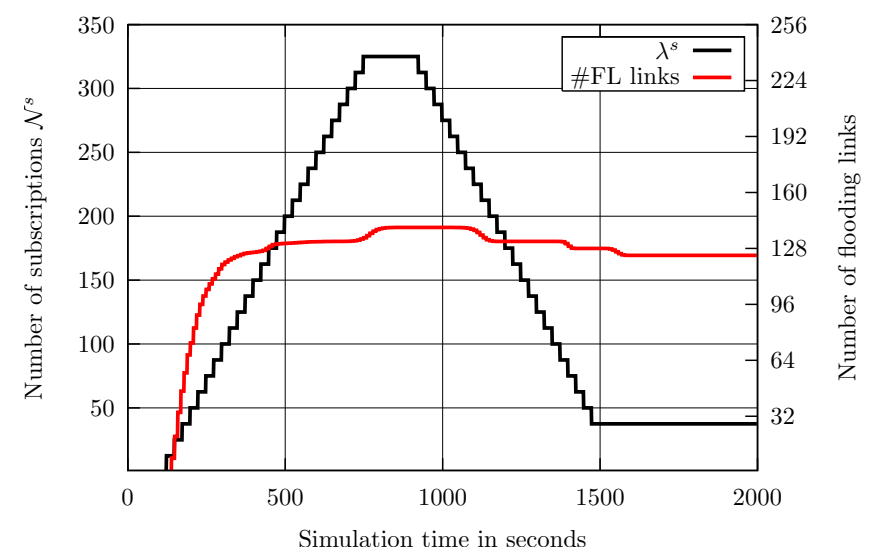

Figure 6.13: Number of flooding links in a dynamic situation

tifications is not higher than if SR is applied. This is because in such cases the subscription traffic is so high that notifications are de facto flooded.

Adding both traffics leads to Figure 6.12 which allows to assess the optimizing behavior of adaptive routing. When only focusing on the curves of FL and SR one easily recognizes that in different situations different routing algorithms are appropriate. In the low interest situation before the step of the subscription birth rate SR is preferable. It generates no traffic in opposite to flooding with $126,000 \mathrm{~s}^{-1}$. In the high interest situation, FL avoids the unnecessary flooding of subscriptions and is, therefore, better. While the traffic of FL does not change, the one of SR has a steep increase until 180,000 messages per second is reached. Adaptive routing is exactly following the idea of choosing the appropriate algorithm. Before the step it remains in SR with all links. After the step the links are stepwise reconfigured to FL. This behavior counteracts the sharp increase of the subscription traffic. The final total traffic is only slightly higher than the notification traffic. Filtering including subscription forwarding is only done on brokers at the very low levels. The figures also show that there are no oscillations after the step of the subscription birth rate.

\subsubsection{Dynamic Situation}

In this experiment we evaluate the dynamics of adaptive routing. As before the setup consists of 127 brokers and we have 1000 different types of notifications that clients can publish or exclusively subscribe to. The type and the originating broker of publications or subscriptions are randomly chosen. The expected overall publication rate is set to $500 \mathrm{~s}^{-1}$. The expected subscription lifetime is one minute. All three processes are stochastic and exhibit exponential distributions. This time, the results have been averaged over 1000 runs leading to smoothened curves.

In Figures 6.13 and 6.14 we compare adaptive routing with homogeneous flooding and simple routing. In the first figure the development of the subscription birth rate is shown. It consists of several parts. We start with an initial subscription birth rate of $0 s^{-1}$. Then, the rate is continuously increased 


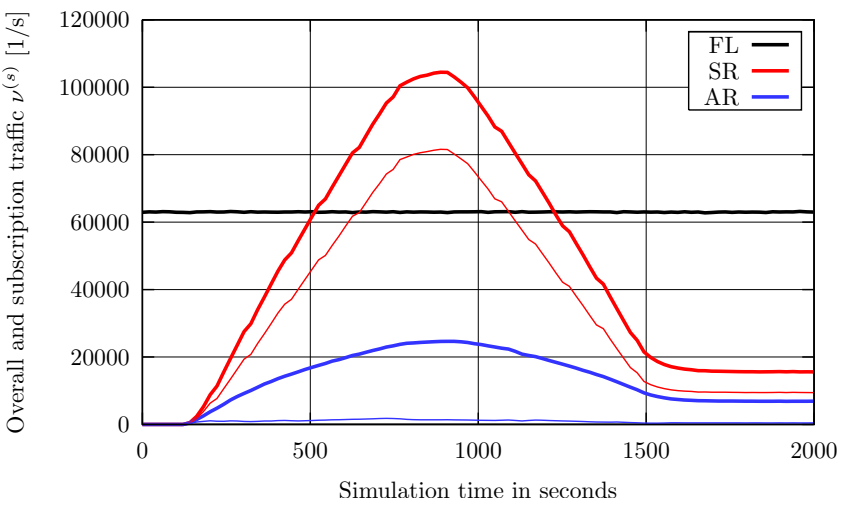

Figure 6.14: Overall traffic in a dynamic situation

up to $325 s^{-1}$. This rate is reached approximately 750 seconds after simulation start. After a short period with a stable rate until about $920 s$, the rate is then continuously reduced down to $37.5 \mathrm{~s}^{-1}$. This value is reached at about $1500 \mathrm{sec}-$ onds. The figure also shows the evolution of the number of flooding links which follows the subscription birth rate with a delay. The reaction time of adaptive routing on the changes is about 130 seconds which can be measured by the difference of the entry points of the stable plateau phase. At a rate of $325 \mathrm{~s}^{-1}$ adaptive routing has reconfigured 140 links which is all links to the top (126) plus three additional levels down $(2+4+8)$. The reduction of the subscription birth rate down to $37.5 \mathrm{~s}^{-1}$ ends up with 124 links which is a hierarchical routing like configuration.

Figure 6.14 compares the evolution of the global traffic. The thick lines show the overall traffic while the thin lines depict the subscription traffic. Clearly, the difference is the notification traffic. As expected in case of flooding, the overall traffic is not changing even if there is no interest in the notifications. With simple routing the traffic is quite low in situations with a low subscription birth rates. For higher rates the subscription traffic more and more dominates the overall traffic so that SR is even worse than FL. In opposite, adaptive routing adapts to the situations by reconfiguring the network. Thus, the relative share of the subscription traffic in the overall traffic is kept low. The notification traffic is only slightly higher than in SR.

Both experiments endorse the practicability of adaptive routing. Since from Section 6.4.2 we already know about the convergence of AR, our focus in the evaluation was to give the reader an idea about the dynamic behavior of it. Beside this simulation we further integrated adaptive routing into REBECA [73] to test its applicability in real-world scenarios. The results were very close to what the simulations showed.

\subsection{Generalizations and Extensions}

In this section we give some important extensions and generalization of adaptive routing. We start with a special form of adaptive routing namely, the adaptive hierarchical routing. Furthermore, we discuss if it is reasonable to use advanced 
routing algorithms, such as covering-based routing or advertisement-based routing, in an adaptive routing manner. Another topic of this section is the composition of adaptive routing and other algorithms including fault-tolerance and adaptive overlay.

\subsubsection{Adaptive Hierarchical Routing}

The starting point for adaptive hierarchical routing is the standard hierarchical routing with a single root broker $R$. A problem that often occurs for the designer is to determine which broker is the best root broker. Therefore, the goal is to make the choice of $R$ adaptive in a way that depending on the current situation an optimal root broker is chosen.

Let the current root broker $R$ be connected to neighbor brokers $B_{i}$ where $B_{i} \in$ $\mathcal{N}(R)$. The routing configurations of the outgoing overlay links $R B_{i}$ are SR and for incoming links $B_{i} R$ it is FL, respectively. For simplification, we summarize the configuration of a single overlay link of both directions by one value, either it is "FL + SR" or we have "SR + FL". For each of the neighbors the root broker can determine the benefit for toggling the routing configuration of the connecting link. The cost measure between the current root broker $R$ and $B_{i}$ (cf. Equation 6.3) is given by:

$$
C_{R i}=c_{R i} \cdot r_{R i}^{s}+c_{i R} \cdot r_{R i}^{s}+c_{i R} \cdot r_{i R}^{n}+c_{R i} \cdot r_{i R}^{s}
$$

By calculating the difference $\Delta C_{R i}$ between the expected value after a reconfiguration $\tilde{C}_{R i}$ and the current value of $C_{R i}$ (cf. Section 6.4), the root broker can determine the neighbor that generates the best benefit (greatest $\Delta C_{R i}$ ) if it becomes root. If this maximum is greater than a predefined threshold $\operatorname{tr}(>0)$, the related link (including the reverse link) is reconfigured. Thereby, the root broker moves to the selected broker. This way the optimal position of $R$ can be determined. The reconfiguration process which moves the root broker from $R$ to $B_{i}$ is an intelligent combination of the reconfigurations in both directions introduced in Section 6.3.3. The following steps have to be done:

1. $R$ decides that $B_{i}$ is the better root broker

(a) $R$ starts to flood notifications towards $B_{i}$

(b) $R$ sends its routing table to $B_{i}$

(c) $R$ starts to send (un)subscriptions towards $B_{i}$

(d) $R$ sends the signal YouAreRoot to $B_{i}$

2. $B_{i}$ receives the signal YouAreRoot
(a) $B_{i}$ activates the filtering towards $R$
(b) $B_{i}$ stops to send (un)subscriptions to $R$
(c) $B_{i}$ acknowledges with the signal YouAreRootAck

3. $R$ receives the signal YouAreRootAck

(a) $B_{i}$ deletes the routing table pointing to $B_{i}$ 
Adaptive hierarchical routing has the advantage compared to standard adaptive routing that only one broker has to be active to check if there are reasonable reconfigurations. Furthermore, the handling of the configurations is much simpler. Especially, there is no need to check if a reconfiguration is possible. On the other hand, only a small subset of all possible hybrid routing configurations is considered. There are only $|\mathcal{B}|$ (size of the topology) configurations for adaptive hierarchical routing which is much less than for the worst case in standard adaptive routing, namely, a line consisting of $|\mathcal{B}|$ brokers with $|\mathcal{B}|^{2}$ possible configurations (cf. Section 6.3.4). Thus, the optimization result may only be suboptimal compared to the more complex algorithm introduced in the latter section. Nevertheless adaptive hierarchical routing can be used in publish/subscribe infrastructures, where only hierarchical routing is supported.

\subsubsection{Advanced Routing Algorithms and Advertisements}

Generally, advanced routing algorithms, like identity-based, covering-based and merging-based routing can be integrated into adaptive routing. As in Section 6.3 , we can transfer the global nature of each algorithm to single links and, thus, mix the algorithms within one overlay. Thus, a routing configuration of an overlay link $r_{i j}$ can also take values, such as CBR for covering-based routing or MBR for merging-based routing. Since each of the routing algorithms guarantees that all necessary routing information in form of subscriptions are disseminated in the system, the correctness of the system is not disturbed. The algorithms only differ in the way they disseminate subscriptions. While SR just floods them, IBR or CBR uses filter similarities to reduce the number of sent subscriptions and, therefore, the number of routing entries. The only restriction we have, is to prohibit that a (notification) flooding link follows a filtering link (no matter if SR, IBR, CBR, MBR) downstream which is a similar criterion as for the special case with FL and SR only in Equation 6.1 resp. 6.2. To support advertisement-based routing an additional configuration like $r_{i j}^{A}$ has to be introduced. Hence, we get a wider definition of hybrid routing algorithms compared to the one in Section 6.3.

Beside this hybrid scheme with advanced routing algorithms, we need an optimization criterion for reconfiguration decisions for an adaptive routing. This criterion contains the current and the expected traffic (cf. Section 6.4). While the measurement of the current traffic is no problem for all algorithms, it is difficult to estimate the expected traffic after a reconfiguration to an advanced routing algorithm. When FL and SR were used, the expected traffic can be estimated by summing up incoming messages from neighbors. This was possible due to the flooding behavior of FL and SR according to notifications resp. subscriptions. The estimation of traffic for advanced algorithms requires to analyze the content of the messages that goes beyond what the routing is in fact doing. For example, for a reconfiguration $r_{i j}=[F L, C B R]$ the estimated subscription traffic $\tilde{r}^{s}$ requires to analyze the covering relation of incoming subscriptions from $\mathcal{S}\left(B_{i} B_{j}\right)$. This way broker $B_{i}$ acts as if it would already use covering-based routing. Since this additional content analysis is hard to compute, it introduces an overhead that has to be considered as part of the costs model. A similar problem occurs when adaptive routing is extended for the use of advertisements. Here, the reconfiguration to advertisement-based routing would require to know the overlapping relation between subscriptions which 


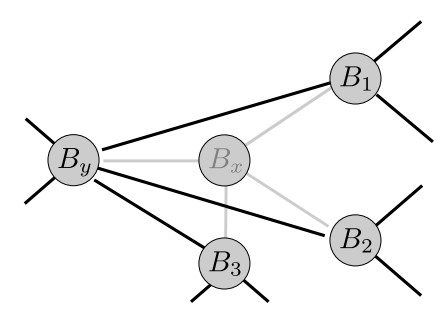

(a) Repair of the topology

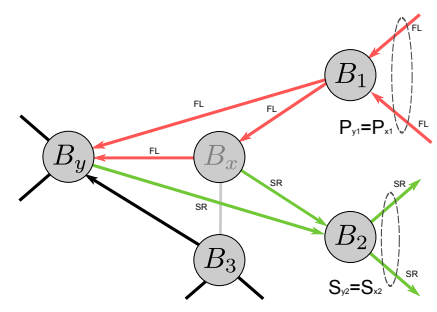

(b) Repair of the routing

Figure 6.15: Fault-tolerance for adaptive routing

is usually not determined in a non-advertisement-based routing. A reverse reconfiguration decision from a more advanced algorithm to a lower one is easier to implement, such as $r_{i j}=[C B R, F L]$. Nevertheless, both reconfiguration directions are needed. To avoid this overhead simple and fast algorithms are needed to roughly estimate the traffic. The search for such algorithms is part of the future work.

\subsubsection{Fault-tolerant Adaptive Routing}

For all kinds of distributed systems, the question of fault-tolerance is immanent due to its nature of distributed system elements. For a sufficiently large system, it is very likely that a fault of a system component occurs. In publish/subscribe one major fault to deal with is a broker fault. A very general class of faults, the Byzantine faults [57], encompass omission failures such as crash failures as well as commission failures such as sending incorrect or inconsistent responses to a request. The latter fault model even contains intelligent responses generated by a (human) attacker to hijack or destroy the system. Following, we focus on a fail-silent crash of a broker. These crashes are assumed to be rare and do occur neither locally nor timely accumulated. Furthermore, we assume that there is an algorithm (short FT) that handles single faults of brokers. This includes the detection of the fault by its connected neighbor brokers, the buffering and repeated delivery of messages to avoid message loss and the reestablishment of a correct routing table to guarantee the deliveries of later publications. The algorithms works in a way that it masks all faults. In opposite to a self-stabilization publish/subscribe system [67], we assume that the algorithm guarantees the correctness of the system even in case of faults. The critical point of the combination of adaptive routing and FT is that we want to preserve the properties of both algorithms. Since we cannot predict when a broker crashes, we must guarantee that in case of a fault at any time, in any state of the adaptive routing algorithm (which also includes the reconfiguration phase) there is a valid and consistent routing configuration.

As described in Chapter 6.3.1, we have to guarantee the correctness of routing, i.e., overlay links with SR must follow SR resp. links with FL must precede FL. Figure 6.15(a) shows a part of an overlay topology consisting of five brokers. After a crash, FT substitutes the faulty broker $B_{x}$ by the substituting broker $B_{y}$. The former neighbor brokers of $B_{x}\left(B_{1}, B_{2}, B_{3}\right)$ reconnect to $B_{y}$. To do this, each broker beforehand knows a substituting broker for its 
neighbors. Formally, the following link modifications take place: $B_{i} B_{x} \rightarrow B_{i} B_{y}$ and $B_{x} B_{i} \rightarrow B_{y} B_{i}$. Additionally, $B_{x} B_{y}$ and $B_{y} B_{x}$ is removed.

To achieve a correct routing configuration, we define that each link $B_{i} B_{y}$ resp. $B_{y} B_{i}$ inherits the routing algorithm (FL or SR) of the corresponding former link $B_{i} B_{x}$ resp. $B_{x} B_{i}$. This can be implemented as part of the reconnection routine of FT. For example, in Figure 6.15(b) $B_{1} B_{y}$ inherits flooding from $B_{1} B_{x}$ and $B_{y} B_{2}$ inherits simple routing from $B_{x} B_{2}$ etc. This strategy is easy to implement since each broker knows its former routing configuration of the link to the failed broker. But more important is that the strategy guarantees that the resulting configuration is valid and does not require routing reconfigurations in other parts of the network. That this proposition is true, can be seen by the way the topology is reconfigured. Actually, broker $B_{x}$ and $B_{y}$ are merged, creating a new broker $B_{y}$ in the overlay tree topology. The new overlay links are "shortcuts" to bypass the failed broker. This guarantees that implications via $\mathcal{P}\left(B_{i} B_{j}\right)$ and $\mathcal{S}\left(B_{i} B_{j}\right)$ are preserved. Since the correctness of a configuration (cf. Equations 6.1 and 6.2) is based on these sets, we can be sure that the configuration is correct after a broker fault has happened and FT has repaired the overlay. After that, FT has to reestablish a correct routing table.

\subsubsection{Fault-tolerant Adaptive Hierarchical Routing.}

Adaptive hierarchical routing (cf. Section 6.6.1) which is based on hierarchical routing has two kinds of brokers, the root broker $R$ and non-root brokers. To make adaptive hierarchical routing fault-tolerant, each non-root broker up to level 2 ( $R$ is located in level 0$)$ must know the parent broker of the parent broker. This broker is the substituting broker in case of a failing parent. While in the standard fault-tolerant adaptive routing each broker observes all its neighbors whether they are still alive, in fault-tolerant adaptive hierarchical routing brokers only observe their parents. This implies that the current root broker $R$ is not involved in the detection of failed neighbors. In case of a fault of a nonroot broker $B_{x}$, the former child brokers of $B_{x}$ connects to the parent of $B_{x}$. As required for hierarchical routing, the new connections are configured to downstream SR and upstream FL. This configuration is equal to the lost connection which is the same behavior as described for the FT algorithm of standard AR. Brokers in level 1 do detect the failure of the $R$. The substituting broker for $R$ is one out of all level 1 brokers. The straightforward way to determine it, is to negotiate it beforehand. If the fail occurs, all brokers on level 1 connect to this broker that will be the new root and set the routing configuration according to the hierarchical routing scheme. As always after a failure, a new substituting broker for the failed broker has to be determined and made public. Due to the asymmetry of the algorithm depending on the relative position to $R$, the FT algorithm for adaptive hierarchical routing seems to be more complex, but all contained "sub-algorithms", including the detection of faults and the reconfiguration of the routing, are easier than the one for standard adaptive routing.

\subsubsection{Composition of Self-optimizing Algorithms}

In Section 4.3, we pointed out that the performance of a publish/subscribe system is determined by its inputs. With the introduced publish/subscribe 


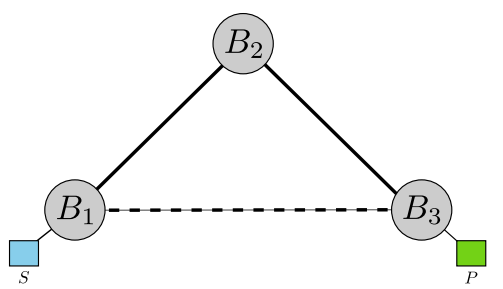

(a) Basic configuration

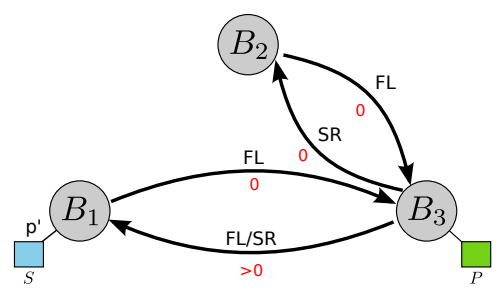

(b) Optimal configuration

Figure 6.16: Composition of self-optimizing algorithms

system model we are able to optimize the configurable inputs, namely routing algorithm and topology (Section 5). In this chapter, we already showed how we can adapt the routing algorithm at runtime to optimize the infrastructure. This way, adaptive routing complements another algorithm that adapts the topology. This algorithm which we call here adaptive overlay or short AO was introduced by Jaeger et al. [45]. Our goal in this section is to discuss the benefits and challenges of a composition of both algorithms following the composition definitions of Richling [84]. Before proceeding to the composition problem, we briefly describe the algorithm presented in [45].

Adaptive Overlay Topologies. Goal of the AO algorithm is to establish shortcuts between brokers when there are pure forwarding brokers [8] on the connecting path between publishers and interested subscribers. The algorithm directly connects brokers with similar interests and removes expensive overlay links. To determine the similarity of interests, each broker manages a cache which stores consumed and published messages in an efficient way by using Bloom filters [13]. This information is periodically sent by each broker to a limited neighborhood. Based on an online heuristic which considers communication costs of links, processing costs of brokers, and patterns in the network's message flows, the brokers agree on beneficial replacements of links. The algorithm works in three phases. In the evaluation phase, the heuristic is applied to find inefficient links and potential replacement candidates. In the consensus phase, the local knowledge of affected nodes is combined to evaluate the benefit of a reconfiguration. The reconfiguration phase finally adapts the topology while ensuring message ordering and avoiding message loss. Figure 6.16(a) shows a very basic example reconfiguration. The initial topology connects a publisher $P$ to subscriber $S$ via the intermediate broker $B_{2}$. AO determines the similarity of interests and will directly connect $B_{1}$ and $B_{3}$. Thereby, the intermediate broker $B_{2}$ is unloaded which solely acts as a simple forwarder, otherwise. In more complex scenarios AO is able to shortcut longer paths. This depends on the size of the limited neighborhood.

Composition. Both algorithms, adaptive routing and adaptive overlay, have the same goal but do use different optimization parameters, the routing algorithm resp. the overlay topology. We use Figure 6.16(a) to explain the benefits of the composition of both algorithms. Therefore, we analyze two situations that characterize different system states as they may occur in dynamic systems. 


\begin{tabular}{|l|l|l|}
\hline Algorithm & Scenario 1 & Scenario 2 \\
\hline \hline Flooding & 0 & $2 p$ \\
\hline Simple Routing & $2 s$ & $2 \cdot\left(s+p^{\prime}\right)$ \\
\hline Adaptive Routing & 0 & $2 \cdot \min \left(p, s+p^{\prime}\right)$ \\
\hline Adaptive Overlay with FL & 0 & $2 p$ \\
\hline Adaptive Overlay with SR & $2 s$ & $2 s+p^{\prime}$ \\
\hline Adaptive Routing+Overlay & 0 & $\min \left(p, s+p^{\prime}\right)$ \\
\hline
\end{tabular}

Table 6.3: Traffics in both scenarios

At the beginning (scenario 1 ) the subscriber at $B_{1}$ subscribes with a subscription birth rate $s$. In this situation the publisher is passive and does not send any publications. After some time, the publisher at $B_{3}$ starts to publish with a publication rate of $p$ (scenario 2). Due to the subscriptions, $S$ is interested in a fraction of the messages published by $P$ which is $p^{\prime}$ with $p^{\prime} \leq p$. Now we analyze the expected total traffic in the network for different algorithms in both scenarios. We start with the standard routing algorithms, i.e., flooding and simple routing. Then, the two single adaptive algorithms, i.e., adaptive routing and adaptive overlay, and, finally their composition is explained. Table 6.3 summarizes the results. When only FL is applied, traffic only appears if there are publishing clients (cf. scenario 2). Since filtering is applied locally, the complete publication rate $p$ is on both overlay links $B_{3} B_{2}$ and $B_{2} B_{1}$. In case of SR, subscriptions are flooded and the overall subscription birth rate is on both links in both situations $(2 s)$. Published notifications are only forwarded if there is an interest $\left(2 p^{\prime}\right)$. When using $\mathrm{AR}$ only, the routing is reconfigured resulting in the optimized configuration. Therefore, the traffic is the minimum of FL and $\mathrm{SR}$ in both situations. With the composition of $\mathrm{AO}+\mathrm{FL}$, the adaptive overlay algorithm would directly connect publisher at $B_{3}$ and subscriber at $B_{1}$ in scenario 2. Due to the usage of flooding this does not reduce the traffic. This drawback is avoided with the composition of AO $+\mathrm{SR}$, where the direct link between $B_{1}$ and $B_{3}$ limits the notification message flow $\left(p^{\prime}\right)$ on this link. Therefore, the traffic is smaller compared to SR only. Finally, the best composition is to combine adaptive routing and adaptive overlay. This composition generally avoids to disseminate unnecessary messages. Therefore, in scenario 2 , the only message flow is between $B_{1}$ and $B_{2}$. Then, either FL generates a flow of $p$ or SR a flow of $s+p^{\prime}$. The resulting configuration with the traffics in red is shown in Figure 6.16(b).

Obviously, the composition of adaptive routing and adaptive overlay results in the smallest overall traffic. Despite this promising results in this example, the composition has its challenges which must be addressed in further research. For that we laid the foundation in [76]. The first challenge is that, unfortunately, both algorithms are not orthogonal but have indirect dependencies. As already mentioned both algorithms try to optimize the system by a control cycle consisting of measuring the current costs, deciding for reconfigurations and finally adapting the system. Since both algorithms use a closely related cost measure based on the message flow, competitive behavior is possible that may lead to oscillation and a performance degradation. The second challenge is that an adaption of the overlay, i.e., the removal of one link and the inser- 
tion of another one, sometimes requires a routing reconfiguration. As described in Section 6.4.2 such overlay reconfigurations may lead to cascading routing reconfigurations which degrade the optimization effect. Furthermore, these cascading routing reconfigurations take a while to be fully active. During this time notifications may be lost which would have been delivered without an overlay reconfiguration. Therefore, if message completeness is required, a more complex coordination protocol is necessary.

\subsection{Discussion}

In this chapter, we presented an optimization algorithm that continually adapts the routing configuration of a content-based publish/subscribe system to reduce its cost. The algorithm is based on so called hybrid routing algorithms allowing to combine different routing algorithms in on broker overlay. Before introducing this approach, the same routing algorithm had to be applied in the whole overlay and it had to be selected before system start. To enable hybrid routing for an offline capacity planning (cf. Chapter 5 ), we showed how it can be integrated into our stochastic system model (cf. Chapter 4).

To enable hybrid routing algorithms for an online optimization, we introduced a reconfiguration scheme which is strictly designed to guarantee the correctness of the publish/subscribe system. Furthermore, we derived an optimization criterion based on estimated traffics to detect beneficial reconfigurations. By bringing all these pieces together, we derived an adaptive routing algorithm which works decentralized with little traffic overhead. The algorithm reduces the costs with respect to the introduced cost model. Our approach is especially advantageous in dynamic scenarios, where the clients' behavior varies over time. However, it is also useful in static scenarios, where the information necessary to determine the optimal routing algorithm is not available before runtime. An important result for adaptive routing is that we could prove that the algorithm always converges to a local cost optimum and in special cases to the global cost optimum which stresses its effectiveness.

Open research questions for the future are mainly related to the composition challenge. Beside the composition with adaptive overlay topologies which must be completed, there are still other algorithms that wait for a combination with adaptive routing, e.g., in fields of distributed event pattern detection [96]. Due to the excepted complexity, each composition approach should be checked by a (formal) calculus. As a long term goal also a generic framework for compositions is interesting but hard to reach. Another research goal is the integration of advanced algorithms and advertisements into adaptive routing requiring new and simple estimates for covering and overlapping relations. Finally, the practice will show if there are algorithmic challenges to avoid unnecessary reconfigurations in highly dynamic situations. Therefore, the prediction of expected traffics and the consideration of costs for reconfigurations, matching and covering must be investigated. 


\section{Chapter 7}

\section{Conclusions and Future Work}

In this thesis, we proposed approaches for modeling and optimizing distributed content-based publish/subscribe systems covering the entire lifecyle of the system. In this final chapter, we summarize the results and present an outlook with ideas and predictions for future work in this field.

\subsection{Conclusions}

The objective of this thesis is to approach the goal of optimizing distributed publish/subscribe systems. This issue is of particular interest since publish/ subscribe systems are more and more used in mission-critical areas inside companies, such as stock trading systems, fraud-detection, etc., where a certain performance level must be met. On the other hand, the economic pressure on companies and more and more ecological issues force designers and operators of the publish/subscribe infrastructures to use their resources efficiently. The balancing of these ambivalent goals cannot be performed by a human operator, because of the large size and complexity of nowadays publish/subscribe systems. As derived in this thesis, optimizations can be done at design time as part of a capacity planning or at runtime in form of self-optimization. Both have their justification, their advantages and drawbacks. Used together they provide a holistic approach to optimize distributed publish/subscribe systems. Summarizing, the contributions of this thesis are twofold. Centered around a new stochastic system model we build a strategy to determine optimized configurations of publish/subscribe systems before they are put into production. Furthermore, we presented ways to render publish/subscribe middlewares selfoptimizing by adapting the applied routing in a self-organizing manner. The binding element for both approaches is the holistic view on the entire lifecyle of the publish/subscribe system. In the following, the content of the individual chapters is summarized.

Chapter 3: Optimizing Publish/Subscribe Systems. Each scientific work needs a formal framework that narrows its topic and formalizes its goals. In 
Chapter 3, we prepared the ground for the modeling and optimization of contentbased publish/subscribe systems. We came up with the identification of two opposing roles in a publish/subscribe system, the client and the operator, with their contrary requirements. Based on this conflict of interests, we derived general optimization objectives that balance requirements of the clients and the operator. We embedded the solution of such optimization objectives into the lifecylce of publish/subscribe systems. Thereby, we identified the necessity of an offline capacity planning based on a system model and an adaptive online algorithms to optimize the system at runtime. Both mentioned approaches were addressed in the following chapters.

Chapter 4: Stochastic System Model. To empower designers to predict a systems' behavior they need a prediction model. In Chapter 4, we presented a stochastic publish/subscribe system model for this purpose. The presented system model contains all major inputs such as the client behavior and the network topology and it provides outputs that contain all important performance measures and costs such as traffics, loads and delays. Due to the stochastic behavior of the clients, the model was derived by the application of probability theory. The interesting insight of the stepwise derivation is a deeper understanding of the benefits of the usage of different routing algorithms. This fact is closely coupled to the determination of forwarding probability for different message types. To model the dynamic aspects of the processing of messages on brokers and their transmission via links we used a queueing theoretic approach. Chapter 4 is the central chapter of this thesis because it lays the foundation of the following capacity planning and also for the self-optimization at runtime. The model is self-contained and can be used to determine outputs for arbitrary inputs, to test the influence of special input changes and for many other purposes. Due to the modularity of the model it is easy to adapt and to extend to requirements that go beyond the assumptions we made in this thesis.

Chapter 5: Capacity Planning. A system model opens up the possibility to perform offline optimizations of systems. In Chapter 5, we built a capacity planning strategy on top of the stochastic system model, i.e., that we dealt with the question how optimized configurations for a publish/subscribe system can be determined. Beside the introduction of a general workflow, we focused on how to efficiently calculate the model. Thereby, we solved numerical challenges that arose from infinite sums and integrations within the models formulas. With the solutions we were able to calculate the results 1000 times faster than with the usage of simulations. To estimate the optimization complexity, we determined a lower and upper bound for it. The chapter was concluded with a case study which stresses the applicability of the presented capacity planning strategy.

Chapter 6: Adaptive Routing. In Chapter 6 we presented adaptive routing, an online optimization algorithm. In opposite to capacity planning, the algorithm improves the performance of a publish/subscribe middleware at runtime by selecting an optimal routing configuration on each link. This algorithm complements other algorithms that optimize the performance such as adaptive overlay. In order to add this self-optimizing behavior we introduced the hybrid routing which allows the combination of different routing algorithms within one 
overlay network based on valid routing configurations. Then, we developed a reconfiguration scheme to reconfigure the routing algorithm on single overlay links. Finally, we derived a local optimization criterion to decide whether a routing reconfiguration is reasonable or not. The algorithm of adaptive routing is completely distributed and it is characterized by its locality, the low introduced overhead and by its convergence. By a mathematical proof we showed that adaptive routing always converges to a local optimum and in many cases even to the global optimum. Additionally, in this chapter we introduced important extensions such as fault-tolerant adaptive routing and the composition with adaptive overlay to get the benefits of both algorithms at the same time.

\subsection{Outlook}

In this thesis, we presented approaches to model and optimize distributed publish/subscribe systems. As for every thesis we had to focus our work by making restricting assumptions. Thus, there is room left for further research in many fields. In the following, we discuss major research questions which result from our work.

\section{Real Data and Simulation}

Publish/subscribe systems often have been used in commercial areas. For example GRYPHON [98] was the monitoring and statistics reporting infrastructure for the Olympic Games in Sydney. Nevertheless, real data of running applications has not been published by the companies because these data are classified as confidential information. To overcome this lack, in papers like [37] or [46] real data from YAhoo Finance or AOL search engine logs are used. Since these applications are not naturally publish/subscribe-based, the authors intelligently convert these data into notifications and subscriptions. Nevertheless, this conversion contains some artificial assumptions that cannot be checked against reality. For example, it is not evident that search queries are equal to subscriptions. Thus, for the further development there is strong need to get (possibly anonymized) real data which also include the spatial distribution of events and subscriptions so that the publish/subscribe research community can increase the significance of the achieved results. Accepted standard benchmarks such as SPECJMS [88] and JMS2009-PS [87] are important first steps in this direction.

\section{Modeling}

The stochastic system model introduced in Chapter 4 and 5 is a big step for performance prediction and capacity planning. Nevertheless, real data will require extensions of the presented model. Real world applications consisting of many interconnected clients do often have correlated and bursty behavior [9] The correlation can easily be observed in applications, where clients functional dependent on the occurrence of special events. The burstiness is a normal case in social networks where special events like news do immediately "effect" the majority of the clients that again publish events in form of comments and further discussions. Therefore, it seems to be necessary to extend the client model in this direction. This extension may have consequences on other submodels beyond the client model. For example, other analysis approaches despite standard 
queueing theory such as the network calculus [28] must be applied. Furthermore, it must be considered if the expected value of performance measures is sufficient or if the model output must be extended by variances and percentiles. To gather reliable delay values the underlay network must be better integrated. A good way would be to use existing TCP/IP models such as $[16,55]$ (just to cite a few) into the publish/subscribe system model to get more accurate results. Finally, it seems to be a good idea to shift from analytical to numerical modeling so that the measured data directly flow in to the calculation.

\section{Composition of Algorithms}

In Chapter 6 we considered the composition of adaptive routing and adaptive overlay in a publish/subscribe middleware. To have a complete self-organizing and optimizing publish/subscribe middleware, additional composition steps are required. Such a middleware would include fault-tolerance and the adaption of routing and overlay topology including the number of brokers. The latter requires an algorithm that decides if a broker is no longer needed because it is a pure forwarder or if a new brokers should be started in case of two very similar message streams on the underlay that could be merged. An initial approach that supports the shrinking of the network size has been proposed in [26]. Despite these algorithmic challenges there is a great demand in middlewares that are open for the composition of publish/subscribe features. First steps in this direction were done by Baldoni et al. [7] and Cugola and Picco [29]. We also presented a promising plug-in based pipeline architecture $[73,74,75]$ that allows a flexible manipulation of the message flows. Nevertheless, the composition of several algorithms remains a big challenge for each special case due to its mostly hidden interdependencies. In the general case, more than a derivation of composition guidelines and composition supporting frameworks does not seem to be soon achievable.

\section{Holistic View}

In Chapter 3, we showed that optimizations may take place in online and offline phase of the life cycle of the publish/subscribe system. For both we presented approaches in the main chapters. What is now needed is to have a general approach that brings both concepts together by building an autonomous system with both components. This way, an even better autonomous publish/subscribe system can be created that from time to time triggers a new capacity planning but continuously adapts the configuration in a self-organized manner. Both mechanism must be coordinated efficiently. Another direction is to merge central algorithms, such as the capacity planning and self-organizing algorithms, such as adaptive routing to one class of algorithms. Each instance of the class is defined by the size of the limited information about its neighbors. This approach which is similar to Schmeck [89] allows a holistic view on distributed algorithms and removes the distinction between centralized and distributed algorithms. In the best case, an algorithm is designed for every size of neighborhood so that it can be adapted according to the requirements. 
Appendix 



\section{Appendix A}

\section{List of Abbreviations and Symbols}

In the whole thesis we introduced a lot of abbreviations to shorten the presentation. Furthermore, in Chapter 4 many symbols have been used within the formulas. In the following tables, we list all abbreviations and symbols arranged in four groups with the section where they have been defined. As before, a symbol with a superscript $x$ means that it depends on the message type $x \in\{n, s, a\}$ namely, notifications, subscriptions and advertisements. 


\begin{tabular}{|c|l|c|}
\hline Abbreviation & Meaning & Sect. \\
\hline \hline ABR/A & advertisement-based routing & 2.4 \\
\hline AL & (arbitrary) routing algorithm & 4.4 .6 \\
\hline AO & adaptive overlay & 6.6 .5 \\
\hline AR & adaptive routing & 6.4 \\
\hline ARC & adaptive routing connect message & 6.4 .2 \\
\hline ARR & adaptive routing reconfiguration request message & 6.4 .2 \\
\hline CBR & covering-based routing & 2.4 \\
\hline FL & flooding & 2.4 \\
\hline FP & false positive notifications & 2.4 \\
\hline FT & fault tolerance & 6.6 .3 \\
\hline HR & hierarchical routing & 2.4 \\
\hline IBR & identity-based routing & 2.4 \\
\hline MBR & merging-based routing & 2.4 \\
\hline SFN(A) & (ack) message: start filtering notifications & 6.3 .3 \\
\hline SR & simple routing & 2.4 \\
\hline SSS(A) & (ack) message: stop sending subscriptions & 6.3 .3 \\
\hline
\end{tabular}

Table A.1: Abbreviations

\begin{tabular}{|c|l|c|}
\hline Symbol & Meaning & Sect. \\
\hline \hline$a$ & an advertisement (message) & 2.2 \\
\hline$B$ & a broker & 2.3 \\
\hline $\mathcal{B}$ & set of all brokers & 2.3 \\
\hline$B_{i} B_{j}$ & an overlay link between broker $B_{i}$ and $B_{j}$ & 2.3 \\
\hline$F, \mathbf{F}$ & a (multidimensional) filter & 2.2 \\
\hline$F L i s t\left(B_{i}, B_{j}\right)$ & list of filters on $B_{i}$ pointing to $B_{j}$ & 2.3 \\
\hline $\bar{l}$ & a physical link & 2.3 \\
\hline$n, \mathbf{n}$ & a (multidimensional) notification & 2.2 \\
\hline$N$ & a single neighboring broker & 4.4 .5 \\
\hline $\mathcal{N}(B)$ & neighbor brokers of $B$ & 4.4 .5 \\
\hline $\mathcal{P}\left(B_{i} B_{j}\right)$ & predecessing brokers of overlay link $B_{i} B_{j}$ & 4.4 .5 \\
\hline$R$ & root broker resp. a rendezvous broker & $2.4,6.3 .1$ \\
\hline$R T(B)$ & routing table of broker $B$ & 2.3 \\
\hline$s$ & a subscription (message) & 2.2 \\
\hline $\mathcal{S}\left(B_{i} B_{j}\right)$ & successing brokers of overlay link $B_{i} B_{j}$ & 4.4 .5 \\
\hline $\mathcal{T}\left(B_{i} B_{j}\right)$ & all brokers of a subtopology rooted by $B_{i} B_{j}$ & 4.4 .5 \\
\hline
\end{tabular}

Table A.2: General symbols 


\begin{tabular}{|c|l|c|}
\hline Symbol & Meaning & Sect. \\
\hline \hline$c(B), c(l)$ & $\begin{array}{l}\text { number of messages that can be handled by broker } B, \text { phys- } \\
\text { ical link } \bar{l}\end{array}$ & 4.4 .9 \\
\hline$\delta(l)$ & physical delay of physical link & 4.6 .3 \\
\hline$\lambda^{x}(B)$ & rate at which messages are generated at a broker & 4.4 .1 \\
\hline$\lambda_{a c c}^{x}\left(B_{i} B_{j}\right)$ & accumulated birth rate of a subtopology $\mathcal{T}\left(B_{i} B_{j}\right)$ & 4.4 .5 \\
\hline$\mu^{x}(B)$ & rate at which messages are removed at broker $B$ & 4.4 .1 \\
\hline$S^{x}(B), S^{x}(l)$ & $\begin{array}{l}\text { service time distribution for messages on broker } B \text {, physical } \\
\text { link } \bar{l}\end{array}$ & 4.6 .3 \\
\hline
\end{tabular}

Table A.3: Symbols for inputs

\begin{tabular}{|c|l|c|}
\hline Symbol & Meaning & Sect. \\
\hline \hline$N^{x}\left(B_{i} B_{j}\right)$ & $\begin{array}{l}\text { number of active subscriptions/advertisements in the } \\
\text { subtopology } \mathcal{T}\left(B_{i} B_{j}\right)\end{array}$ & 4.4 .5 \\
\hline$p^{\text {match }}(k)$ & $\begin{array}{l}\text { probability that a notification is matched by a least } \\
\text { one subscription.if there are } k \text { active subscriptions }\end{array}$ & 4.4 .7 \\
\hline$p^{\neg c o v}(k)$ & $\begin{array}{l}\text { probability that a subscription is not covered by any } \\
\text { other subscriptions.if there are } k \text { active subscriptions }\end{array}$ & 4.4 .7 \\
\hline$p^{x}(k)$ & $\begin{array}{l}\text { probability that a message is forwarded if there are } \\
k \text { active subscriptions }\end{array}$ & 4.4 .7 \\
\hline$P^{x}\left(B_{i} B_{j}\right)$ & $\begin{array}{l}\text { probability that a message is forwarded via overlay } \\
\text { link } B_{i} B_{j}\end{array}$ & 4.4 .7 \\
\hline$P^{\bar{n}}\left(B_{i} B_{j}\right)$ & $\begin{array}{l}\text { probability that a forwarded notification which is not } \\
\text { needed (false positive) via overlay link } B_{i} B_{j}\end{array}$ & 4.4 .7 \\
\hline$P_{a c c}^{x}\left(B_{i} B_{j}\right)$ & $\begin{array}{l}\text { expected number of forwardings of a message if a } \\
\text { message is published at } B_{i} \text { and further forwarded to } \\
B_{j}\end{array}$ & 4.4 .8 \\
\hline$q^{x}\left(B_{i} B_{j}\right)$ & $\begin{array}{l}\text { probability that there are k active things in the } \\
\text { subtopology } \mathcal{T}\left(B_{i} B_{j}\right)\end{array}$ & 4.4 .1 \\
\hline$x_{l}^{x}(B)$ & $\begin{array}{l}\text { number of occupied local notification/subscription } \\
\text { routing entries at broker } B\end{array}$ & 4.4 .6 \\
\hline
\end{tabular}

Table A.4: Symbols for probabilities 


\begin{tabular}{|c|l|c|}
\hline Symbol & Meaning & Sect. \\
\hline \hline$\Delta^{x}$ & $\begin{array}{l}\text { expected delay for the worst path a message might } \\
\text { traverse }\end{array}$ & 4.4 .9 \\
\hline$D(B), D\left(B_{i} B_{j}\right)$ & $\begin{array}{l}\text { message delay caused by broker } B \text {, by overlay link } \\
B_{i} B_{j}\end{array}$ & 4.4 .9 \\
\hline$\nu_{\text {out }}^{x}\left(B_{i} B_{j}\right), \nu_{\text {out }}^{x}(l)$ & $\begin{array}{l}\text { message traffic passing through overlay link } B_{i} B_{j}, \\
\text { physical link } \bar{l}\end{array}$ & 4.4 .8 \\
\hline$\nu_{\text {out }}^{x}(B)$ & generated traffic of broker $B$ & 4.4 .8 \\
\hline$\nu_{\text {in }}^{x}(B)$ & received traffic of broker $B$ & 4.4 .8 \\
\hline$\nu^{x}$ & global traffic in the network & 4.4 .8 \\
\hline$U(B), U(l)$ & utilization of a broker $B$, a physical link $l$ & 4.4 .9 \\
\hline$W(B), W(l)$ & $\begin{array}{l}\text { waiting time for messages at broker } B \text { or a physical } \\
\text { link } \bar{l}\end{array}$ & 4.4 .9 \\
\hline$X^{x}\left(B_{i} B_{j}\right), X^{x}\left(B_{i}\right), X^{x}$ & $\begin{array}{l}\text { expected number of remote notification/subscription } \\
\text { routing entries of broker } B_{i} \text { pointing to } B_{j}, \text { of broker } \\
B_{i}, \text { of the entire network }\end{array}$ & \\
\hline
\end{tabular}

Table A.5: Symbols for outputs including traffic, utilization and delay 


\section{Appendix B}

\section{Routing Configurations of a Binary Tree}

In Figure B.1 all correct routing configurations of a complete binary tree consisting of three levels is depicted. Similar to the plots in Section 6.3.4 the reconfiguration paths are shown and completed levels are marked in gray. 


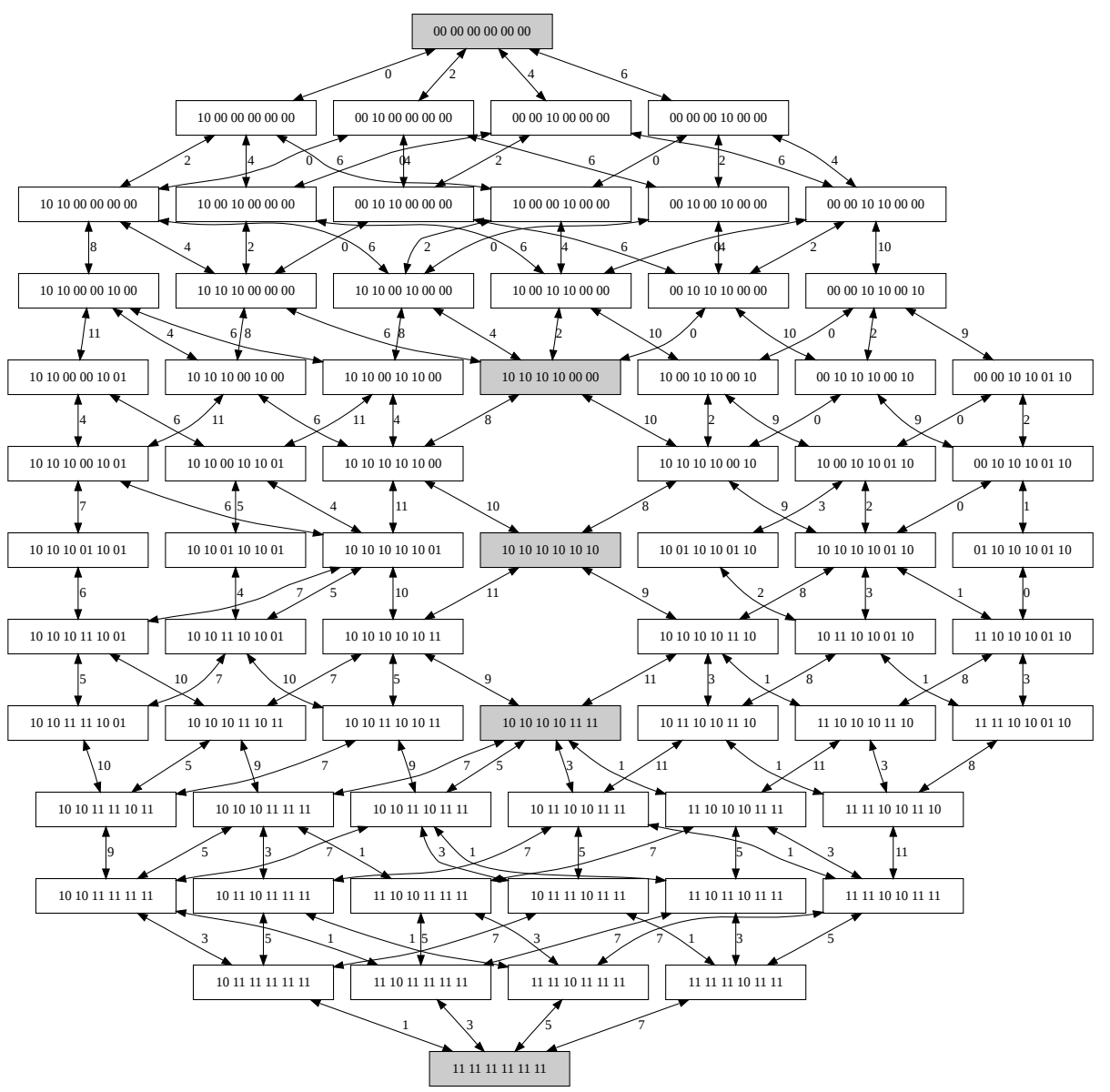

Figure B.1: Routing configurations of a binary tree with three levels 


\section{Bibliography}

[1] Aguilera, M., Strom, R., Sturman, D., Astley, M., and ChanDRA, T. Matching events in a content-based subscription system. In Proceedings of the 18th ACM Symposium on Principles of Distributed Computing (PODC'g9) (1999), pp. 53-61.

[2] Al-Hakeem, M. S., And Heiss, H.-U. Adaptive scheduling for staged applications: The case of multiple processing units. In Internet and Web Applications and Services (ICIW), 2010 Fifth International Conference on (May 2010), pp. 51-60.

[3] Bacon, J., And Pietzuch, P., Eds. DEBS '10: Fourth ACM International Conference on Distributed Event-Based Systems 2010 (New York, NY, USA, 2010), ACM.

[4] Bal, H. E. Programming Distributed Systems. Silicon Press, Summit, NJ, 1990.

[5] Baldoni, R., Beraldi, R., Piergiovanni, S. T., And Virgillito, A. Measuring notification loss in publish/subscribe communication systems. In Proc. of 10th IEEE Pacific Rim International Symposium on Dependable Computing (2004), pp. 84-93.

[6] Baldoni, R., Beraldi, R., Piergiovanni, S. T., And Virgillito, A. On the modelling of publish/subscribe communication systems. Concurrency and Computation: Practice and Experience 17, 12 (Oct. 2005), $1471-1495$.

[7] Baldoni, R., Beraldi, R., Querzoni, L., And Virgillito, A. A selforganizing crash-resilient topology management system for content-based publish/subscribe. In Proceedings of the 3rd International Workshop on Distributed Event-Based Systems (DEBS'04) (May 2004), A. Carzaniga and P. Fenkam, Eds., IEEE, pp. 3-8.

[8] Baldoni, R., Beraldi, R., Querzoni, L., and Virgillito, A. Efficient publish/subscribe through a self-organizing broker overlay and its application to SIENA. The Computer Journal 50, 4 (July 2007), 444-459.

[9] Barabasi, A.-L. The origin of bursts and heavy tails in human dynamics. Nature 435, 7039 (May 2005), 207-211. 
[10] Bianchi, S., Felber, P., And Gradinariu, M. Content-based publish/subscribe using distributed R-trees. In Proceedings of 13th International Euro-Par Conference (EuroPar 2007) (Aug. 2007), A.-M. Kermarrec, L. Bougé, and T. Priol, Eds., vol. 4641 of Lecture Notes in Computer Science, Springer, pp. 537-548.

[11] Bickson, D., Hoch, E. N., NaAman, N., And Tock, Y. A hybrid multicast-unicast infrastructure for efficient publish-subscribe in enterprise networks. In Proceedings of the 3rd Annual Haifa Experimental Systems Conference (New York, NY, USA, 2010), SYSTOR '10, ACM.

[12] Bittner, S. General Boolean Expressions in Publish-Subscribe Systems. PhD thesis, The University of Waikato, Department of Computer Science, May 2008.

[13] Bloom, B. H. Space/time trade-offs in hash coding with allowable errors. Commun. ACM 13 (July 1970), 422-426.

[14] Bricconi, G., Nitto, E. D., And Tracanella, E. Issues in analyzing the behavior of event dispatching systems. In Proceedings of the 10th International Workshop on Software Specification and Design (IWSSD'00) (2000), IEEE Computer Society, pp. 95-103.

[15] Briones, J. A., Koldehofe, B., And Rothermel, K. SPINE: Publish/subscribe for wireless mesh networks through self-managed intersecting paths. In Proc of the 8th IEEE International Conference on Innovative Internet Community Systems (I2CS 2008) (2008).

[16] Bu, T., AND Towsley, D. Fixed point approximations for TCP behavior in an AQM network. In Proceedings of the 2001 ACM SIGMETRICS international conference on Measurement and modeling of computer systems (New York, NY, USA, 2001), SIGMETRICS '01, ACM, pp. 216-225.

[17] Burke, P. J. The output of a queueing system. Operations Research 4 (1956), 699-704.

[18] Campailla, A., Chaki, S., Clarke, E., Jha, S., and Veith, H. Efficient filtering in publish-subscribe systems using binary decision diagrams. In Proceedings of the 23rd International Conference on Software Engineering (ICSE'01) (Toronto, Canada, May 2001), pp. 443-452.

[19] Carzaniga, A. Architectures for an Event Notification Service Scalable to Wide-area Networks. PhD thesis, Politecnico di Milano, Milano, Italy, Dec. 1998.

[20] Carzaniga, A., And Hall, C. P. Content-based communication: a research agenda. In Wohlstadter [103], pp. 2-8. Invited Paper.

[21] Carzaniga, A., Rosenblum, D., And Wolf, A. L. Design and evaluation of a wide-area event notification service. ACM Transactions on Computer Systems 19, 3 (2001), 332-383. 
[22] Carzaniga, A., And Wolf, A. L. Forwarding in a content-based network. In Proceedings of the 2003 Conference on Applications, Technologies, Architectures, and Protocols for Computer Communications (SIGCOMM'03) (Aug. 2003), A. Feldmann, M. Zitterbart, J. Crowcroft, and D. Wetherall, Eds., ACM Press, pp. 163-174.

[23] Castelli, S., Costa, P., And Picco, G. P. Modeling the communication costs of content-based routing: The case of subscription forwarding. In Jacobsen et al. [42], pp. 38-49.

[24] Castelli, S., Costa, P., And Picco, G. P. HyperCBR: Large-scale content-based routing in a multidimensional space. In Proceedings of the 27th IEEE International Conference on Computer Communications (INFOCOM 08) (Phoenix, AZ, USA, Apr. 2008), IEEE Press, pp. 1714-1722.

[25] Chang, E. J. H. Echo algorithms: Depth parallel operations on general graphs. Software Engineering, IEEE Transactions on SE-8, 4 (July 1982), 391-401.

[26] Cheung, A., And Jacobsen, H.-A. Green resource allocation algorithms for publish/subscribe systems. In Distributed Computing Systems (ICDCS), 2011 31st International Conference on (june 2011), pp. 812 $-823$.

[27] Cheung, A. K. Y., And Jacobsen, H.-A. Publisher placement algorithms in content-based publish/subscribe. In Distributed Computing Systems (ICDCS), 2010 IEEE 30th International Conference on (june 2010), pp. $653-664$.

[28] Cruz, R. L. A calculus for network delay. II. network analysis. Information Theory, IEEE Transactions on 37, 1 (Jan. 1991), 132-141.

[29] Cugola, G., and Picco, G. P. REDS: A reconfigurable dispatching system. In Wohlstadter [103], pp. 9-16.

[30] Deering, S., And Hinden, R. Internet protocol, version 6 (IPv6) specification, 1998.

[31] Eugster, P., Felber, P., Guerraoui, R., and Kermarrec, A.-M. The many faces of publish/subscribe. ACM Computing Surveys 35, 2 (2003), 114-131.

[32] Fidler, E., Jacobsen, H.-A., Li, G., and Mankovski, S. The PADRES distributed publish/subscribe system. In In 8th International Conference on Feature Interactions in Telecommunications and Software Systems (2005), pp. 12-30.

[33] Fiege, L., Mezini, M., Mühl, G., and Buchmann, A. Engineering event-based systems with scopes. In Proceedings of the European Conference on Object-Oriented Programming (ECOOP) (Malaga, Spain, June 2002), B. Magnusson, Ed., vol. 2374 of LNCS, Springer-Verlag, pp. 309333. 
[34] Fraser, A. S. Simulation of genetic systems by automatic digital computers. Australian Journal of Biological Sciences 10 (1957).

[35] Gelernter, D. Generative communication in Linda. ACM Transactions on Programming Languages and Systems 7, 1 (Jan. 1985), 80-112.

[36] Grassé, P.-P. La reconstruction du nid et les coordinations interindividuelles chez bellicosi-termes natalensis et cubitermes sp. la theorie de la stigmergie: Essai d'interprétation des termites constructeurs. Insectes Sociaux 6 (1959), 41-83.

[37] Gupta, A., Sahin, O. D., Agrawal, D., and Abbadi, A. E. Meghdoot: Content-based publish/subscribe over P2P networks. In Proceedings of the 5th ACM/IFIP/USENIX International Conference on Middleware (MIDDLEWARE'04) (Oct. 2004), H.-A. Jacobsen, Ed., Springer-Verlag, pp. $254-273$.

[38] He, F., Baresi, L., Ghezzi, C., And Spoletini, P. Formal analysis of publish-subscribe systems by probabilistic timed automata. In 27th IFIP WG 6.1 Intl. Conf. on Formal Techniques for Networked and Distributed Systems (2007), vol. 4574 of LNCS, pp. 247-262.

[39] Heiss, H.-U., And Dormanns, M. Partitioning and mapping of parallel programs by self-organization. Concurrency: Practice and Experience 8, 9 (1996), 685-706.

[40] Henjes, R. Performance Evaluation of Publish/Subscribe Middleware Architectures. PhD thesis, University of Würzburg, Nov. 2010.

[41] Hinze, A., Sachs, K., And Buchmann, A. Event-based applications and enabling technologies (keynote). In Proc. of DEBS 2009 (July 2009).

[42] Jacobsen, H.-A., Mühl, G., And Jaeger, M. A., Eds. Proceedings of the Inaugural Conference on Distributed Event-Based Systems (DEBS'07) (New York, NY, USA, June 2007), ACM Press.

[43] Jaeger, M. A. Self-Organizing Publish/Subscribe. IEEE Distributed Systems Online 7, 2 (Feb. 2006). art. no. 0602-o2003.

[44] Jaeger, M. A., ANd Mühl, G. Stochastic analysis and comparison of self-stabilizing routing algorithms for publish/subscribe systems. In Proceedings of the 13th IEEE/ACM International Symposium on Modeling, Analysis and Simulation of Computer and Telecommunication Systems (MASCOTS'05) (Sept. 2005), G. F. Riley, R. Fujimoto, and H. Karatza, Eds., IEEE Press, pp. 471-479.

[45] Jaeger, M. A., Parzyjegla, H., Mühl, G., and Herrmann, K. Selforganizing broker topologies for publish/subscribe systems. In Proceedings of the 22nd Annual ACM Symposium on Applied Computing (SAC'O7) (Mar. 2007), L. M. Liebrock, Ed., ACM, pp. 543-550.

[46] Jerzak, Z. XSiena: The Content-Based Publish/Subscribe System. PhD thesis, Technische Universität Dresden, Dresden, Germany, 2009. 
[47] Jerzak, Z., and Fetzer, C. Bloom filter based routing for contentbased publish/subscribe. In Proceedings of the second international conference on Distributed event-based systems (New York, NY, USA, 2008), R. Baldoni, Ed., ACM, pp. 71-81.

[48] Kendall, D. G. Stochastic processes occurring in the theory of queues and their analysis by the method of the imbedded markov chain. Annals of Mathematical Statistics 24 (1953), 338-354.

[49] Kephart, J. O., and Chess, D. M. The vision of autonomic computing. Computer 36, 1 (Jan. 2003), 41-50.

[50] Kirkpatrick, S., Gelatt, C. D., and Vecchi, M. P. Optimization by Simulated Annealing. Science, Number 4598, 13 May 1983220 (1983), 671-680.

[51] Klatt, B., Rathfelder, C., And Kounev, S. Integration of eventbased communication in the palladio software quality prediction framework. In QoSA-ISARCS '11: Proceedings of the joint ACM SIGSOFT conference - QoSA and ACM SIGSOFT symposium - ISARCS on Quality of software architectures - QoSA and architecting critical systems ISARCS (New York, NY, USA, 2011), ACM, pp. 43-53.

[52] KLeinrock, L. Queueing Systems; Theory, vol. 1. John Wiley and Sons, New York, 1975.

[53] Kleinrock, L. Queueing Systems; Computer Applications, vol. 2. John Wiley and Sons, New York, 1976.

[54] Kounev, S., Sachs, K., Bacon, J., and Buchmann, A. A methodology for performance modeling of distributed event-based systems. In Proc. of the 11th IEEE Intl. Symposium on Object/Component/Service-oriented Real-time Distributed Computing (May 2008), pp. 13-22.

[55] Lakshman, T. V., and Madhow, U. The performance of TCP/IP for networks with high bandwidth-delay products and random loss, 1997.

[56] LAmport, L. A new approach to proving the correctness of multiprocess programs. ACM Transactions on Programming Languages and Systems (TOPLAS) 1, 1 (1979), 84-97.

[57] Lamport, L., Shostak, R., and Pease, M. The byzantine generals problem. ACM Trans. Program. Lang. Syst. 4 (July 1982), 382-401.

[58] Li, G., Hou, S., and Jacobsen, H.-A. XML routing in data dissemination networks. In Proceedings of 23rd International Conference on Data Engineering(ICDE) (2007), IEEE.

[59] Li, G., Muthusamy, V., and Jacobsen, H.-A. Adaptive contentbased routing in general overlay topologies. In Middleware '08: Proceedings of the 9th ACM/IFIP/USENIX International Conference on Middleware (New York, NY, USA, Nov. 2008), ACM/IFIP/USENIX, SpringerVerlag New York, Inc., pp. 1-21. 
[60] Luckham, D. The Power of Events: An Introduction to Complex Event Processing in Distributed Enterprise Systems. Addison-Wesley Professional, 2002.

[61] Luckham, D., And Schulte, R. Event processing glossary - version 1.1, July 2008

[62] Lunze, J. Regelungstechnik 1, 5. ed. Springer, Berlin, Sept. 2005.

[63] Margara, A., and Cugola, G. High performance content-based matching using GPUs. In Proceedings of the 5th ACM International Conference on Distributed Event-Based Systems 2011 (New York, NY, USA, 2011), O. Etzion, Ed., ACM, pp. 183-194.

[64] Medina, A., Lakhina, A., Matta, I., and Byers, J. BRITE: An approach to universal topology generation. In Proceedings of the International Workshop on Modeling, Analysis and Simulation of Computer and Telecommunications Systems (MASCOTS'01) (Aug. 2001), IEEE Computer Society, pp. 346-353.

[65] MüHL, G. Large-Scale Content-Based Publish/Subscribe Systems. PhD thesis, Darmstadt University of Technology, Sept. 2002.

[66] Mühl, G., Fiege, L., And Pietzuch, P. R. Distributed Event-Based Systems. Springer-Verlag, Aug. 2006.

[67] Mühl, G., Jaeger, M. A., Herrmann, K., Weis, T., Fiege, L., and Ulbrich, A. Self-stabilizing publish/subscribe systems: Algorithms and evaluation. In Proceedings of the 11th International Conference on Parallel Processing (Euro-Par 2005) (2005), J. C. Cunha and P. D. Medeiros, Eds., vol. 3648 of Lecture Notes in Computer Science (LNCS), Springer-Verlag, pp. $664-674$.

[68] Mühl, G., Schröter, A., Parzyjegla, H., Kounev, S., and RichLING, J. Stochastic analysis of hierarchical publish/subscribe systems. In Euro-Par '09: Proceedings of the 15th International Euro-Par Conference on Parallel Processing (Berlin, Heidelberg, 2009), Springer-Verlag, pp. $97-109$.

[69] Mühl, G., Werner, M., Jaeger, M. A., Herrmann, K., And PARzyjegla, $\mathrm{H}$. On the definitions of self-managing and self-organizing systems. In KiVS 2007 Workshop: Selbstorganisierende, Adaptive, Kontextsensitive verteilte Systeme (SAKS'07) (Mar. 2007), T. Braun, G. Carle, and B. Stiller, Eds., ITG/GI, VDE Verlag, pp. 291-301.

[70] Müller-Schloer, C., Schmeck, H., And Ungerer, T. Organic Computing - A Paradigm Shift for Complex Systems, vol. 1 of Autonomic Systems. Springer Basel, 2011.

[71] Oh, S., Pallickara, S. L., Ko, S. H., Kim, J. H., and Fox, G. Cost model and adaptive scheme for publish/subscribe systems on mobile grid environments. In 5th Intl. Conf. on Computational Science (2005), vol. 3516 of $L N C S$, pp. $275-278$. 
[72] Pankajakshan, A., and RaO, S. Modeling a publish/subscribe system as a multi-commodity transportation problem. In Systems Conference (SysCon), 2012 IEEE International (march 2012), pp. 1 -6.

[73] Parzyjegla, H., Graff, D., Schröter, A., Richling, J., And MüHL, G. Design and Implementation of the Rebeca Publish/Subscribe Middleware. Springer Verlag, Sept. 2010, pp. 124-140.

[74] Parzyjegla, H., Schröter, A., Busse, A., Graff, D., SchepelJAnski, A., Richling, J., Werner, M., And MüHL, G. REBECA - Eine autonome Publish/Subscribe Middleware. Praxis der Informationsverarbeitung und Kommunikation (PIK) 34, 3 (2011), 135-147.

[75] Parzyjegla, H., Schröter, A., Graff, D., Busse, A., SchepelJanski, A., Richling, J., Werner, M., And Mühl, G. Autonomy features and feature composition in REBECA. In 8th International Conference on Autonomic Computing (ICAC 2011) (June 2011), ACM. (accepted for publication).

[76] Parzyjegla, H., Schröter, A., Seib, E., Holzapfel, S., Wander, M., Richling, J., Wacker, A., Heiss, H.-U., MüHl, G., And Weis, T. Model-driven development of self-organising control applications. In Organic Computing - A Paradigm Shift for Complex Systems, C. MüllerSchloer, H. Schmeck, and T. Ungerer, Eds., vol. 1 of Autonomic Systems. Springer Basel, 2011, pp. 131-144. 10.1007/978-3-0348-0130-0_8.

[77] Paton, N. W., and Díaz, O. Active database systems. ACM Computing Surveys 31, 1 (1999), 63-103.

[78] Paxson, V. E. Measurements and Analysis of End-to-End Internet Dynamics. PhD thesis, EECS Department, University of California, Berkeley, June 1997.

[79] Pietzuch, P. R. Hermes: A Scalable Event-Based Middleware. PhD thesis, Computer Laboratory, Queens' College, University of Cambridge, Feb. 2004.

[80] Pietzuch, P. R., And Bacon, J. Peer-to-peer overlay broker networks in an event-based middleware. In Proceedings of the 2nd International Workshop on Distributed Event-Based Systems (DEBS'03) (June 2003), H.-A. Jacobsen, Ed., ACM Press, pp. 1-8.

[81] Pongthawornkamol, T., and Nahrstedt, K. Towards timeliness and reliability analysis of distributed content-based publish/subscribe systems over best-effort networks. Tech. rep., University of Illinois at UrbanaChampaign, 2009.

[82] Pongthawornkamol, T., Nahrstedt, K., and Wang, G. Probabilistic qoS modeling for reliability/timeliness prediction in distributed content-based publish/subscribe systems over best-effort networks. In Proceeding of the 7th international conference on Autonomic computing (New York, NY, USA, 2010), ICAC '10, ACM, pp. 185-194. 
[83] Rathfelder, C., Kounev, S., and Evans, D. Capacity planning for event-based systems using automated performance predictions. In 26th IEEE/ACM International Conference on Automated Software Engineering (ASE 2011), Lawrence, KS, USA, November 6-10, 2011 (2011), P. Alexander, C. S. Pasareanu, and J. G. Hosking, Eds., IEEE, pp. 352361.

[84] Richling, J. Komponierbarkeit eingebetteter Echtzeitsysteme. PhD thesis, Humboldt-Universität zu Berlin, Berlin, Germany, Feb. 2006.

[85] Rowstron, A., Kermarrec, A.-M., Castro, M., and Druschel, P. Scribe: The design of a large-scale event notification infrastructure. In Networked Group Communication, J. Crowcroft and M. Hofmann, Eds., vol. 2233 of Lecture Notes in Computer Science. Springer Berlin / Heidelberg, 2001, pp. 30-43. 10.1007/3-540-45546-9_3.

[86] Royce, W. W. Managing the development of large software systems: Concepts and techniques. WESCON Technical Papers 14 (1970).

[87] Sachs, K., Appel, S., Kounev, S., and Buchmann, A. Benchmarking publish/subscribe-based messaging systems. In Database Systems for Advanced Applications: DASFAA 2010 International Workshops: BenchmarXío (2010), LNCS, Springer-Verlag.

[88] Sachs, K., And Kounev, S. Workload scenario for SPECjms "Supermarket Supply Chain". Tech. Rep. DVS06-1, SPEC OSG Java Subcommittee, 2006.

[89] Schmeck, H., Müller-Schloer, C., Çakar, E., Mnif, M., and Richter, U. Adaptivity and self-organization in organic computing systems. ACM Trans. Auton. Adapt. Syst. 5 (Sept. 2010), 1-32.

[90] Schmeiser, B., AND TAAFfe, M. R. Time-dependent queueing network approximations as simulation external control variates. Statistical analysis for stochastic modeling and simulation with applications to manufacturing. Purdue University, Dept. of Statistics, 1993.

[91] Schröter, A. Modeling and optimizing content-based publish/subscribe systems. In MDS '09: Proceedings of the 6th Middleware Doctoral Symposium (New York, NY, USA, 2009), P. Triantafillou, Ed., ACM, pp. 1-6.

[92] Schröter, A., Graff, D., Mühl, G., Richling, J., and ParzyJEGLA, H. Self-optimizing hybrid routing in publish/subscribe systems. In Proceedings of the 20th International Workshop on Distributed Systems: Operations and Management, DSOM 2009 (2009), vol. 5841 of Lecture Notes in Computer Science, Springer, pp. 111-122.

[93] Schröter, A., Mühl, G., Kounev, S., Parzyjegla, H., and RichLING, J. Stochastic performance analysis and capacity planning of publish/subscribe systems. In Bacon and Pietzuch [3], pp. 258-269.

[94] Schröter, A., Mühl, G., Richling, J., and Parzyjegla, H. Adaptive routing in publish/subscribe systems using hybrid routing algorithms. In ARM '08: Proceedings of the 7th workshop on Reflective and adaptive middleware (New York, NY, USA, 2008), ACM, pp. 51-52. 
[95] Schulte, R. A real-time enterprise is event-driven. Tech. rep., Gartner Research, Sept. 2002.

[96] Seib, E., Parzyjegla, H., and Mühl, G. Distributed composite event detection in publish/subscribe networks - a case for self-organization. Workshops der wissenschaftlichen Konferenz Kommunikation in verteilten Systemen 2011 (WowKiVS 2011), Electronic Communications of the EASST 37 (Mar. 2011).

[97] Sleator, D. D., and Tarjan, R. E. Amortized efficiency of list update and paging rules. Commun. ACM 28, 2 (Feb. 1985), 202-208.

[98] Strom, R. Gryphon: An information flow based approach to message brokering. In Proceedings of the 9th International Symposium on Software Reliability Engineering (ISSRE'98) (Nov. 1998).

[99] Strom, R., Banavar, G., Chandra, T., Kaplan, M., Miller, K., Mukherjee, B., Sturman, D., And Ward, M. Gryphon: An information flow based approach to message brokering. In IN PROCEEDINGS OF THE INTERNATIONAL SYMPOSIUM ON SOFTWARE RELIABILITY ENGINEERING (1998).

[100] Tanenbaum, A. S. Modern Operating Systems, 2 ed. Prentice Hall, NJ 07458, 2001.

[101] Terpstra, W. W., Behnel, S., Fiege, L., Zeidler, A., And BuchMANN, A. A peer-to-peer approach to content-based publish/subscribe. In In Proceedings of the 2nd International Workshop on Distributed EventBased Systems (DEBS'03) (San Diego, CA, USA, June 2003), A. Jacobsen, Ed., ACM Press.

[102] van Vliet, F., Boucherie, R., and de Graaf, M. Content-based routing in networks with time-fluctuating request rates. In Network Control and Optimization (2009), R. Núñez-Queija and J. Resing, Eds., Lecture Notes in Computer Science, Springer Berlin / Heidelberg, pp. 75-90. 10.1007/978-3-642-10406-0_6.

[103] Wohlstadter, E., Ed. ACM Press.

[104] Wun, A., Petrovic, M., and Jacobsen, H.-A. A System for Semantic Data Fusion in Sensor Networks. In Jacobsen et al. [42], pp. 75-79. Demo paper.

[105] Yan, T., AND Garcia-Molina, H. Index structures for selective dissemination of information under the Boolean model. ACM Transactions on Database Systems (TODS) 19, 2 (June 1994), 332-364.

[106] Zhang, C., Krishnamurthy, A., Wang, R. Y., and Singh, J. P. Combining flexibility and scalability in a peer-to-peer publish/subscribe system. In 6th International Middleware Conference (MIDDLEWARE 2005) (Grenoble, France, Nov. 2005), G. Alfonso, Ed., vol. 3790 of Lecture Notes in Computer Science, Springer, pp. 102-123. 
